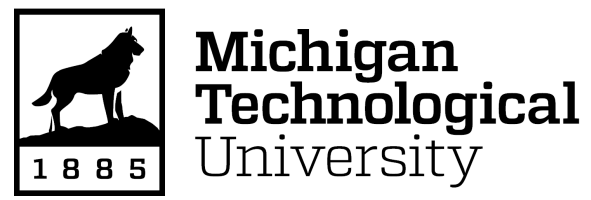

Michigan Technological University Digital Commons @ Michigan Tech

Characterizing the physicochemical properties of TDP-43 protein and Acetylated Amyloid $\boldsymbol{\beta}$ peptides to discern its role in neurodegenerative diseases

Rashmi Adhikari

Michigan Technological University, rashmia@mtu.edu

Copyright 2019 Rashmi Adhikari

Recommended Citation

Adhikari, Rashmi, "Characterizing the physicochemical properties of TDP-43 protein and Acetylated Amyloid $\beta$ peptides to discern its role in neurodegenerative diseases", Open Access Dissertation, Michigan Technological University, 2019.

https://doi.org/10.37099/mtu.dc.etdr/891

Follow this and additional works at: https://digitalcommons.mtu.edu/etdr 


\title{
CHARACTERIZING THE PHYSICOCHEMICAL PROPERTIES OF TDP-43 PROTEIN AND ACETYLATED AMYLOID $\beta$ PEPTIDES TO DISCERN ITS ROLE IN NEURODEGENERATIVE DISEASES
}

\section{By}

Rashmi Adhikari

\author{
A DISSERTATION \\ Submitted in partial fulfillment of the requirements for the degree of \\ DOCTOR OF PHILOSOPHY \\ In Biochemistry and Molecular Biology
}

MICHIGAN TECHNOLOGICAL UNIVERSITY

2019

(C) 2019 Rashmi Adhikari 
This dissertation has been approved in partial fulfillment of the requirements for the Degree of DOCTOR OF PHILOSOPHY in Biochemistry and Molecular Biology

Program.

Department of Chemistry

Dissertation Advisor: Dr. Ashutosh Tiwari (Chemistry)

Committee Member: Dr. Haiying Liu (Chemistry)

Committee Member: Dr. Rupali Datta (Biological Sciences)

Committee Member: Dr. Xiaoqing Tang (Biological Sciences)

Interim Department Chair: Dr. John Jaszczak (Chemistry) 


\section{Table of Contents}

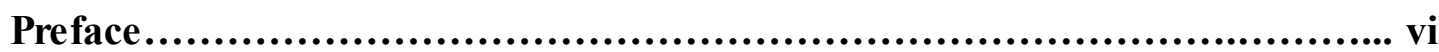

Acknowledgement....................................................................viii

Abstract..........................................................................

Chapter $1 \quad$ Background............................................................ 1

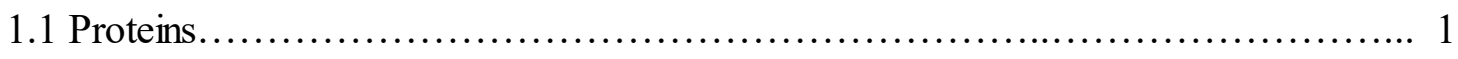

1.2 Physical forces involved in protein folding.............................. 3

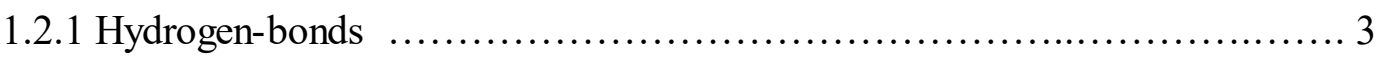

1.2.2 Electrostatic Interactions........................................ 4

1.2.3 Hydrophobic Interaction....................................... 5

1.2.4 Vander Waals Interaction........................................... 5

1.2.5 Intrins ic propensities.......................................... 6

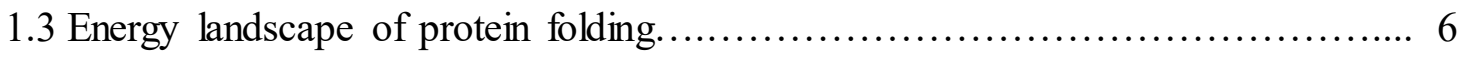

1.4 Disulfide bonds...................................................... 8

1.5 Posttranslational modification (PTM) of proteins......................... 9

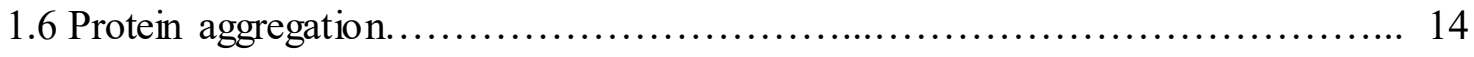

1.6.1 Types of protein aggregates.................................... 15

1.7 Protein aggregation in neurodegenerative diseases......................... 19

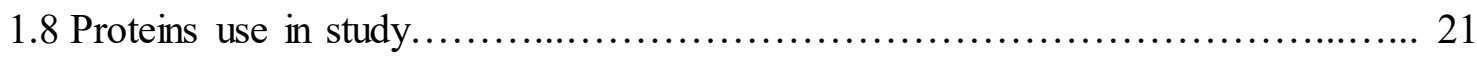

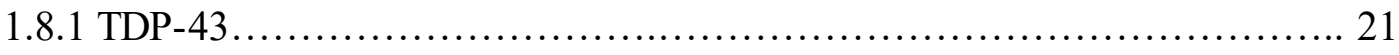

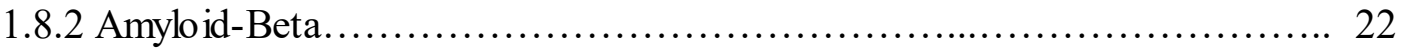

1.9 Motivation......................................................... 23

1.10 References....................................................... 26 
Chapter 2 Methods............................................................... 32

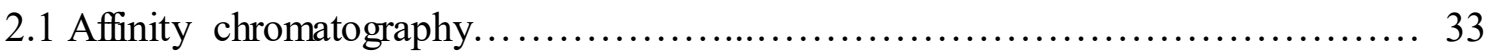

2.2 Polyacrylamide gel electrophoresis (PAGE)..................................... 34

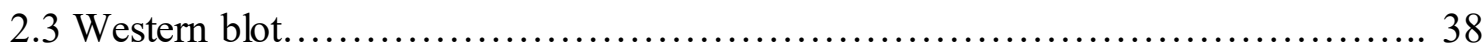

2.4 Scanning Electron Microscopy .............................................. 40

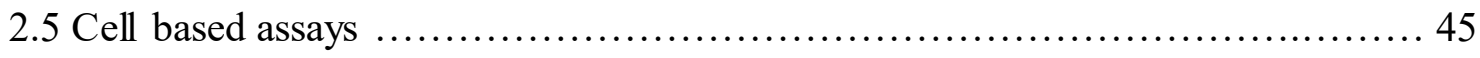

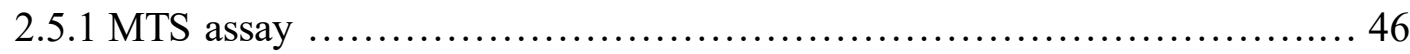

2.5.2 Lactate dehydrogenase (LDH) assay .................................. 49

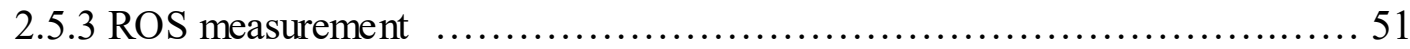

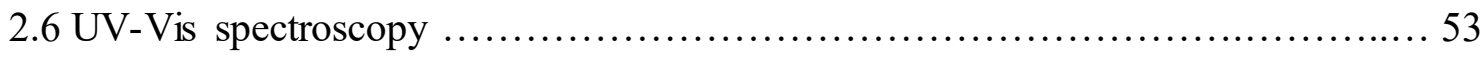

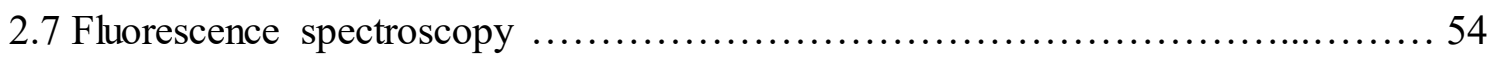

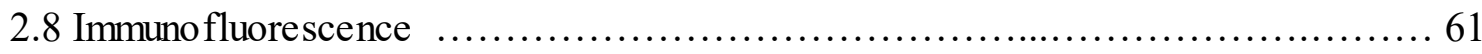

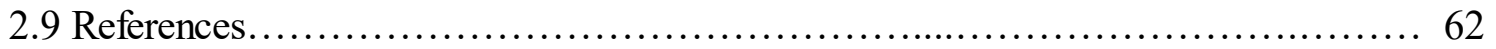

Chapter 3 Characterizing the physicochemical properties of TDP-43 protein to discem its role in neurodegenerative diseases................................... 65

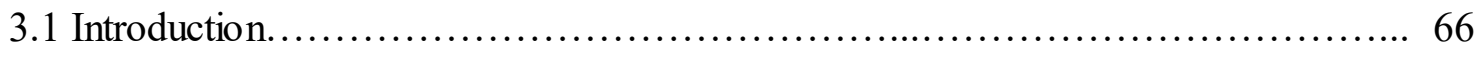

3.2 Materials and Methods..................................................... 80

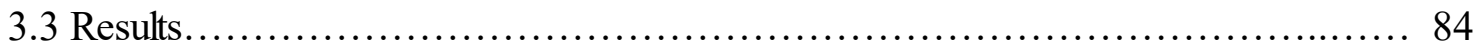

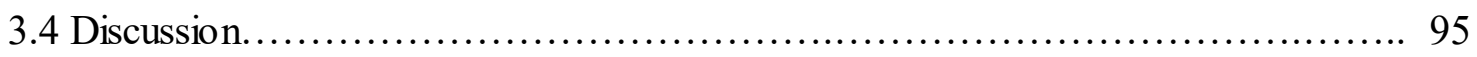

3.5 Conclusion.............................................................. 100

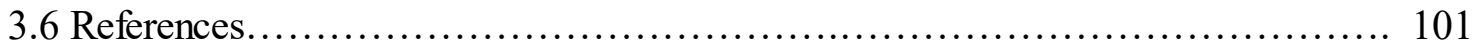


Chapter 4 Acetylation at lysine residues modulates A $\mathbf{\beta 4 2}$ aggregates structure and

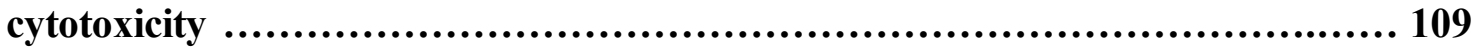

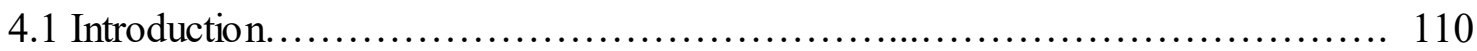

4.2 Methods........................................................... 112

4.3 Results.............................................................. 118

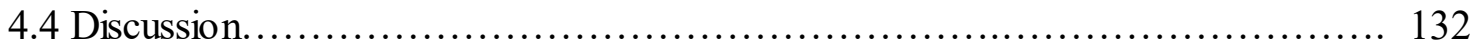

4.5 Conclusion........................................................ 139

4.6 References............................................................ 140

Chapter 5 Summary and Future Perspective................................... 154

Appendix A. Supporting Information for Chapter 3..........................158

Appendix B. Supporting Information for Chapter 4..........................177

Copyright permission.............................................................231 


\section{Preface}

All the contents in chapter $1,2,3,4$, and 5 were written by the author of this dissertation and revised by Dr. Ashutosh Tiwari. This dissertation is the collection of manuscript submitted to peer-review journal for publication and article under preparation. The initial ideas of all research projects in this dissertation were conceived by Dr. Ashutosh Tiwari.

Chapter 3 is based on multi-author article which is currently under preparation. The author of this dissertation carried out the expression, purification and characterization of protein and is responsible for all data collection, analysis and writing of the manuscript. The cloning part was carried out by former postdoctoral fellow Dr. Jagadeesh Janjanam and Dr. Ashutosh Tiwari. Dr. Colina Dutta contributed to this work by helping with initial expression of the TDP-43 protein. Dr. Ashutosh Tiwari revised the manuscript and is the corresponding Author.

Chapter 4 is based on multi-author article that is submitted to Scientific Reports for publication. This work is a shared authorship between author of this dissertation and Dr. $\mathrm{Mu}$ Yang. The Tht fluorescence measurements were carried out by Dr. Yang and was involved in preparation of the initial manuscript. The author of this dissertation carried out ANS, Bis-ANS, SEM, MTS, LDH, DCFH-DA experiments. Primary neuronal cell cultures were prepared by Dr. Zhiying Shan and Immunostaining experiments were conducted by the author of this dissertation. Ms. Wafa FA Alharbi assisted in Immunostaining experiment. Dr. Colina Dutta carried out the SEM imaging reported in submitted paper. SEM images in this chapter are work of the author of this dissertation. All computational work and associated writing were carried out by Professor Ravindra Pandey and Dr. 
Nabanita Saikia. Dr. Ashutosh Tiwari wrote and revised the manuscript and is the corresponding author. 


\section{Acknowledgements}

Foremost, I would like to present my sincere gratitude to my advisor, Dr. Ashutosh Tiwari, for his continuous guidance, immense support, encouragement and motivation throughout my $\mathrm{PhD}$ study. Without his faith on me I would not have made this far. I could not imagine having better advisor than him.

I would like to acknowledge Dr. Haiying Liu, Dr. Rupali Datta and Dr. Xiaoqing Tang for their time, support and suggestions on my $\mathrm{PhD}$ dissertation.

I am grateful to my research collaborators Dr. Haiying Liu, Dr. Ravindra Pandey, Dr. Nabanita Saikia and Dr. Zhiying Shan for their efforts, suggestion and helpful discussion.

I am fortunate to have opportunity to work with highly skilled seniors in my lab. I express sincere thanks to Dr. Jagadeesh Janjanam, Dr. Mu Yang, Dr. Nethaniah Dorh and Dr. Colina Dutta for their guidance and support.

I would like to express sincere thanks to my teaching supervisors: Ms. Kelley Smith, Ms. Lorri A. Reilly, Ms. Aparna Pandey, Dr. Laleh Vahdat, and Dr. Nabanita Saikia, for their supervision and continuous encouragement during my graduate study teaching span.

I am thankful to the Department of Chemistry, Biochemistry and Molecular Biology program for providing financial assistance throughout this study. The help from all staff in Department of Chemistry including Ms. Charlene Page, Ms. Denise Laux, Mr. Dean Seppala, Mr. Jerry Lutz, Ms. Celine Grace, Mr. Don Wareham and Ms. Kimberly McMullan is highly appreciated. 
Other various financial supports are also greatly thankful, including Ray E. and Eleanor K. Cross Endowed Graduate Fellowship (Department of Chemistry, Michigan Technological University, Summer 2018), Doctoral Finishing Fellowship (Graduate School, Michigan Technological University, Fall 2018), Travel Grants from Graduate Student Government (GSG) and American Chemical Society (UP Local Section, Marquette) for 257rd ACS National Meeting, 2019.

I would like to express my sincere gratitude to my family for their unconditional love, support, trust and encouragement. Many thanks to my sister, Ms. Rijana Adhikari for her support, suggestions and valuable presence. At last, special thanks to a person, who guided me like a mentor, helped as a colleague, and always backed me up like a true friend. Thank you, my dear husband, nothing would have been possible without you being on my side. Your unconditional support throughout these years made me the person I am today. I am very fortunate to have you in my life. 


\begin{abstract}
Proteins are the biological macromolecules central to most cellular functions. The native structure of protein determines its function and any perturbations such as mutation, changes in $\mathrm{pH}$, macromolecular crowding, oxidative stress, may promote protein misfolding and/or aggregation. Protein misfolding or aggregation can contribute to diseases either through loss of proteins' critical function or by gain of toxic function. Among the various proteins associated with neurodegeneration, trans-active response (TAR) DNA binding protein 43 (TDP-43) has sparked interest in researchers due to its diverse role in several neurodegenerative diseases like Amyotrophic Lateral Sclerosis (ALS), Frontotemporal Lobar degeneration (FTLD), Alzheimer's, Parkinson's and Huntington's disease. Most of the reported work on TDP-43 protein have been carried out on truncated version of the protein from prokaryotic source due to difficulty in expressing and purifying the full-length protein. Another protein, amyloid $\beta$ peptides role in Alzheimer's disease has been studied in great detail. However, the role posttranslational modifications play in $A \beta$ related toxicity has not been fully explored. In this dissertation, I address these two major gaps as follows: 1) I have successfully purified full-length TDP-43 protein using a eukaryotic source and carried out some physicochemical characterization. Now this full-length protein which is from eukaryotic source can be used to better understand proteins role in health and disease. 2) I explored the physicochemical properties of acetylated Amyloid $\beta$ peptides including its effects on modulating toxicity in SH-SY5Y cell lines (neuroblastoma) and primary neuronal cultures. This work underscores the importance of posttranslational modification such as acetylation of $A \beta$ peptides on toxicity as well as aggregate morphology which will lead to better drug designs in future.
\end{abstract}




\section{Chapter 1 Background}

\subsection{Proteins}

Proteins, this word is familiar to us, since our childhood we learnt that proteins are the building block of our body. Made up of 20 different amino acids, proteins play role in performing a wide variety of biological function. In order to be functionally active, protein needs to fold into its native, three-dimensional structure. In addition, about $20-30 \%$ of proteins in eukaryotic cells lack their three-dimensional structure and remains folded once they interact with their binding partners. The newly synthesized proteins have a greater chance of being misfolded leading to aggregation and forms a toxic species under the cellular conditions ${ }^{1}$. Cells have several quality control measures like molecular chaperones, which guide the correct de novo folding or refolding of the protein, and help to prevent aggregation of non-native proteins. Ubiquitin-proteasome system (UPS) and Phagosomelysosome system helps to degrade the misfolded proteins (Figure 1) ${ }^{2,3}$. Despite having cellular control measures, there is evidence showing the presence of misfolded and aggregated proteins appearing at late stage of life in several neurological and systemic diseases. The most likely cause of this is decline in the proteostasis capacity of a cell with the aging of an individual. Studies have suggested the several genetic and environmental factors can contribute to protein misfolding and aggregation. Change in conformation of normal protein leads to protein destabilization, which eventually leads to disease condition. Mutation, changes in metal ions, $\mathrm{pH}$, macromolecular crowding, oxidative stress or high concentration of misfolded protein might drive the process of protein misfolding and aggregation $^{3}$. In order to strengthen the understanding of protein folding, misfolding, 
aggregation and toxicity, I will discuss the different forces involved in protein folding, posttranslational modifications, factors leading to misfolding and aggregation. In addition, I will highlight how morphology/nature of aggregates of disease proteins along with key proteins contribute to cytotoxicity leading to neurodegenerative diseases. This will help underscore the importance of my two main body of work included as chapters in this dissertation.

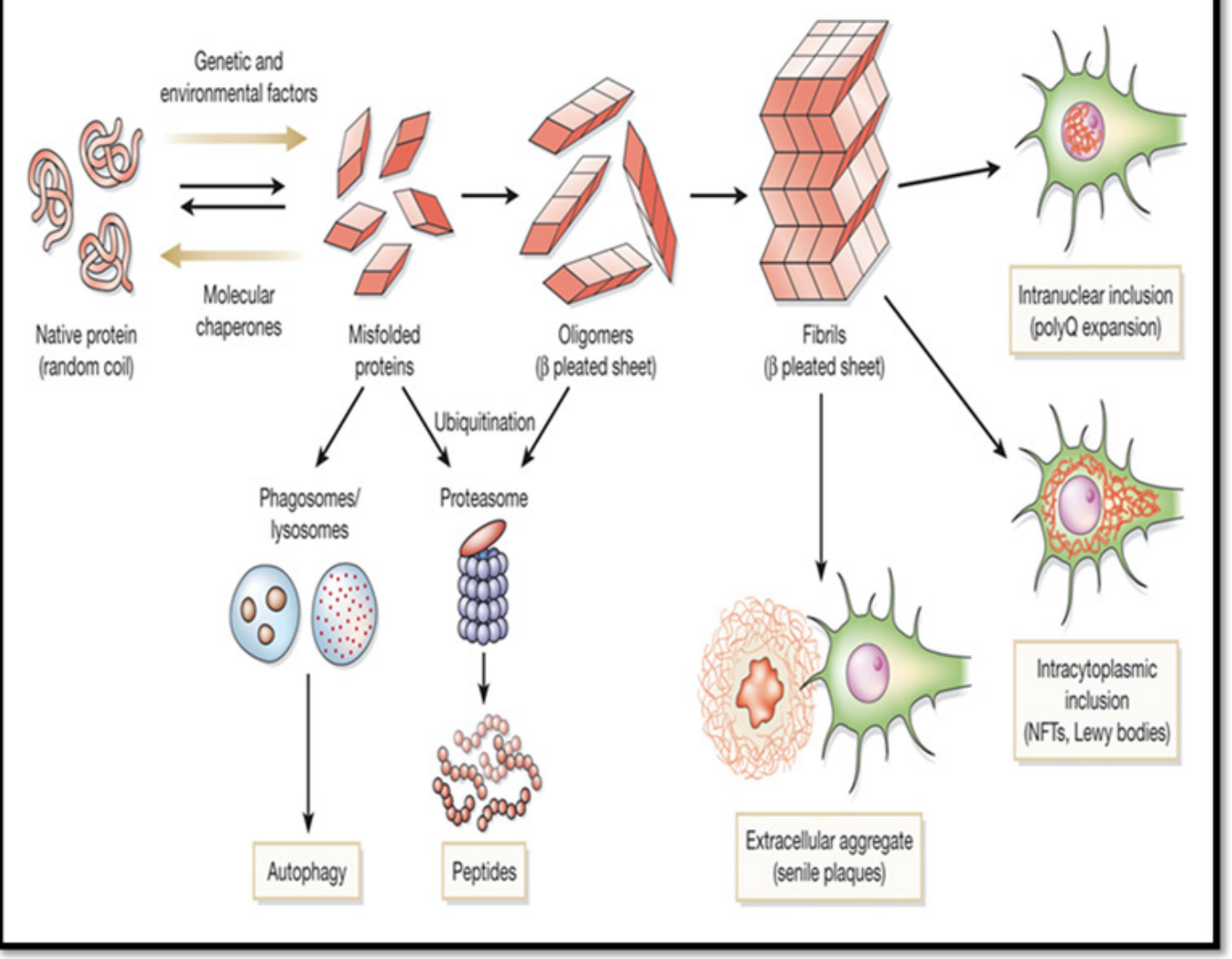

Figure 1: Representation of the Protein misfolding and fibrillization. Genetic and environmental factors might play the role in the process of aggregation thus resulting into the deposition of aggregated proteins in the nucleus, cytoplasm and extracellular space. Several cellular quality control systems like molecular chaperones, the ubiquitin 
proteasome system and the phagosome-lysosome system facilitate in the reduction of accumulation of misfolded proteins. Reprinted with permission from Forman, S. et.al. $2004^{2}$

\subsection{Physical forces involved in protein folding}

Protein folding is a complex process that has been studied for more than a century. As every protein requires very unique set of parameters for its correct folding, protein folding problem has intrigued researchers for a very long time. Researchers are interested in understanding how native structure of proteins acquires its well folded 3D structure. A protein starts as a sequence, like a chain of amino acids that rapidly folds, residues come close ( 2 to $7 \AA$; short/medium-range distance) to each other held by non-covalent forces, forming the native structure of a protein. Now, a substantial amount of information about a protein can be obtained from Protein Databank (PDB), which has collection of more than 100,000 protein structures. Interestingly, proteins are only marginally stable at room temperature and its stability can be affected by the weak non-covalent interactions. This makes understanding of these different types of molecular interactions involved in protein stability very important. The major non-covalent forces in a protein are: Hydrogen bonds, Van der Waals interaction, Electrostatic interactions, and hydrophobic interactions that contribute to protein folding and stability. In the following paragraphs, I will discuss briefly about each of these forces.

\subsubsection{Hydrogen bonds}

During protein folding, due to contact with water $70 \%$ of the peptide groups and $65 \%$ of polar side chains are buried in the interior of the protein ${ }^{4}$. Hydrogen bonds are formed by 
the interaction of the hydrogen atoms that are covalently bonded to the electronegative atoms (usually $\mathrm{N}, \mathrm{O}, \mathrm{S}$ ). Hydrogen bonding provides the rigidity to the protein structure. Both polar side-chains as well as carbonyl oxygen and $\mathrm{N}$-amide proton from peptide bond can take part in H-bonding. H-bond also provides the structural integrity to the $\alpha$-helix and $\beta$-sheet conformations of proteins. The strength of the hydrogen bond is usually in the range of $2-10 \mathrm{kca} / \mathrm{mol}$ which depends on the orientation as well as electronegativity of the bonding atoms. The concept of $\mathrm{H}$-bonding as the dominant force in protein folding was first suggested by Mirsky and Pauling (1936). They introduced the possibility of formation of $\mathrm{H}$-bond between carbonyl $\mathrm{C}=0$ and amide $\mathrm{NH}$ groups of the peptide backbone ${ }^{5}$.

\subsubsection{Electrostatic interactions}

The earliest known protein denaturants were acids and bases, hence researchers assumed that electrostatic interactions are the forces responsible for protein folding. Any process that is driven electrostatically is dependent on $\mathrm{pH} /$ ionic strength. The total charge on the protein is determined by the protein whereas salt determines the extent of interaction. Linderstrom-Lang was the first person to propose the quantitative model of electrostatic interactions for native proteins in $1924^{5}$. Electrostatic interactions can affect protein stability in different ways. One such way is due to the non-specific repulsion interaction which arises when the protein is extreme charged. The native protein can be destabilized by increasing the charge of the solution either by increasing acidity or basicity of the solution. This results in the increase in the charge density in the folded protein compared to the unfolded one leading into its destabilization due to greater charge repulsion. For this reason, when the protein is at its isoelectric point, there is no effect of electrostatic 
interaction on protein stability. Another way that affect the protein stability includes specific charge interactions. Ion pairing or the salt bridge is observed when the side chains of two oppositely charged amino acids are in close proximity to each other. As the charge in the protein increases, the destabilization of folded protein takes place but the ion pairing can stabilize the protein. Earlier in 1930's the dominant force in protein folding was considered to be ion pairing. Studies shows that ion pairing affects the protein stability but is not considered as the dominant force as much of the charges are centered on the surface $6,7$.

\subsubsection{Hydrophobic interactions}

Hydrophobic interactions occurs in a protein as a result of nonpolar side chain of amino acids as well as other nonpolar solutes prefer to cluster in nonpolar environment rather than a polar solvent. The formation of bond reduces the interaction of nonpolar residues with water thus making the interaction highly favorable. During folding of the protein, the hydrophobic side chains of amino acids are buried down in the interior of the protein core and polar amino acids are not found in the interior of the protein ${ }^{8}$. The case for importance of hydrophobic interaction in protein folding was first made strong by Kauzmann.

\subsubsection{Van der Waals Interactions}

Van der Waals interactions are the weak interactions with the stabilization energies of 0.4 to $4.0 \mathrm{~kJ} / \mathrm{mol}$. Interactions with the fixed and induced dipoles results into Van der Waals attractions. These kinds of interactions occur largely in proteins and contribute significantly to stability of protein. 


\subsubsection{Intrinsic propensities}

Intrinsic propensities do not relate to single type of force but is related to preference of certain confirmation by di-or tri-peptides, their size or sequence, which can arise from the local interactions. The stability of the long polypeptides chains in aqueous solutions is due to intrinsic propensities. For instance, As the length of the polypeptide chain increases the stability of the helix is also increased. Although while considering the free energy from individual residue is small but summing up the free energy over many residues results into strong force that favors helix formation. The intrinsic propensities is also responsible for protein confirmation when it folds.

\subsection{Energy landscape of protein folding}

Getting back into the history of protein folding, the researchers assumed that the folding of the protein takes place in distinct pathways through particular intermediate states. Figure 2 shows the classical pathways for protein folding. The graphical representation of protein folding in the funnel shape is the generic representation that is applicable not only to proteins but also to RNA and compact polymers. It represents that protein folds through several intermediate pathways. Studies by protein denaturation experiments suggested protein exist as a two-state model, i.e. at equilibrium they either can exist as fully native or fully unfolded structure ${ }^{4}$. Later, Ptitsyn and coworkers found that several destabilizing conditions such as low $\mathrm{pH}$ can lead to protein with expanded form, with loose tertiary structure but still having the secondary structure in considerable amount which was called as molten globule $4,5-7,9$. 
The large polypeptide chain has complex folding pathways and different models have been developed to describe the pathways. Among those model one describes that the folding process is hierarchical in which the local secondary structures are formed first where the amino acids sequence fold into $\alpha$ and $\beta$ sheets. Next, the super secondary structures are formed which occurs due to the long-range interactions, like two $\alpha$-helices come together and form stable structure. This process takes place until the folding process of a protein is completed. In another model, the folding is mediated by the hydrophobic interactions among non-polar residues where the polypeptide is collapsed spontaneously into compact state. At this stage, there is a high content of secondary structures, however much of the side-chain of amino acids are not fixed. The resultant from the hydrophobic collapse are often termed as molten globule state. While considering how protein folds, most of the proteins folds by incorporating the features of both models. Proteins may take a different route for folding that results into same end point with several folding intermediates.

The process of protein folding can be viewed as a free-energy funnel. The unfolded states contain high degree of conformational entropy as well as free energy. The narrowing of funnel takes place as the folding process proceeds where the decrease of entropy takes place. The semistable intermediates are represented by the small depressions that are found along the side of the funnel and they slow down the process of folding. At the end of the funnel, a native confirmation is present where other folding intermediates are reduced ${ }^{10}$. 

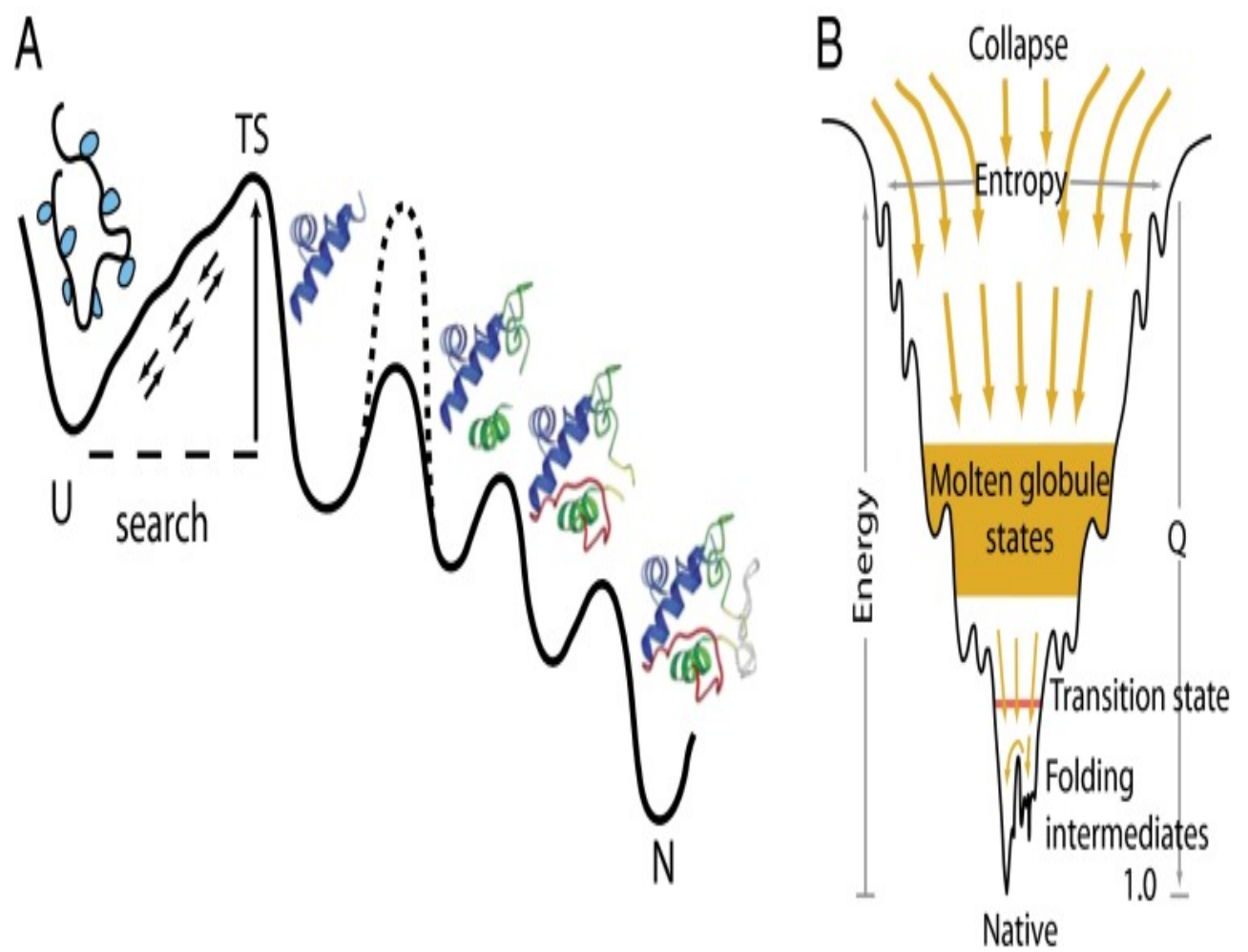

Figure 2: Folding pathways of a protein A. Classic approach of a protein folding pathway B. Funnel-shaped landscape of a protein folding9 ${ }^{9}$. Reprinted with permission from Englander and Mayne. Proc Natl Acad Sci U S A. 2014 Nov 11; 111(45): 1587315880201597.

\subsection{Disulfide bonds}

Disulfide bond is present in most proteins and it ensures the correct folding of proteins under native conditions. Disulfide bonds occupy $15 \%$ of the human proteome and are present mainly in secreted protein where it makes up to $65 \%$. Next to peptide bond, the common covalent link between the amino acids in the protein is the disulfide bond. Disulfide bonds are known to decrease the entropy of the unfolded state thereby stabilizes the protein thermodynamically. The presence of disulfide bonds are found in $55 \%$ of the 
pathologic amyloid forming proteins underscoring the importance of disulfide bonds in aggregation kinetics, nature of aggregates, as well as its role in cellular toxicity. For proper folding of protein into its native structure, many proteins require the correct formation of disulfide bonds. Protein misfolding results when there is abnormal reduction/formation of disulfide bonds. Disulfide bonds are involved in proteins involved in neurodegeneration. Until date, the role of disulfide bonds have been studied and found to be important in three proteins involved in neurodegeneration: prion, SOD1 and Tau. The folding/misfolding pathway can thus be traced by determining the thiol-disulfide status in the protein. For example, the folding/misfolding is regulated by thiol-disulfide status in case of SOD1 (Cu, Zn-superoxide dismutase). The stabilization of matured SOD1 is achieved by the formation of intramolecular disulfide bonds whereas the reduction of these disulfide bonds leads to the misfolding and aggregation of SOD1 due to a decrease in conformational stability of a protein. Therefore, the disulfide bonds play important role in familial form of amyotrophic lateral sclerosis (fALS) .

\subsection{Posttranslational modification (PTM) of proteins}

Synthesis of the proteins occurs during a process of 'translation.' The chemical changes that protein may have after the process of translation or concomitant with translation is commonly known as posttranslational modifications. Posttranslational modifications is present in both prokaryotes and eukaryotes. However, the modifications are present more in eukaryotic cells where the enzyme constitutes $5 \%$ of the genome that carry out posttranslational modification of protein. Post-translation modification can be enzymatic and non-enzymatic depending on its attachment of the chemical group to amino-acid side 
chain $^{11}$.The most common modifications includes the formation of disulfide bonds, cleavage of the precursor proteins or the addition or removal of low molecular weight groups covalently, which results into modifications like acetylation, biotinylation, amidation, deamidation, farnesylation, formylation, cysteinylation, geranylgeranylation, glutathionylation, glycation, glycosylation, hydroxylation, methylation, mono-ADPribosylation, myristoylation, oxidation, palmitoylation, phosphorylation, poly(ADPribosyl)ation, stearoylation, or sulfation $\mathrm{n}^{1-16}$.

Table 1: Posttranslational modifications and their target amino acids with enzyme involved.

\begin{tabular}{|l|l|l|}
\hline Amino acid residue(s) & Protein/Enzyme involved & Post-translation modification \\
Tyrosine, Serine, & Kinases, phosphatases & Phosphorylation \\
\hline Lysine & Ubiquitin-activating & Ubiquitination \\
\hline Lysine & enzymes, Ubiquitin ligases & \\
\hline Lysine, Arginine & Acetyltransferases, & Acetylation \\
\hline deacetylases & Methylation \\
\hline
\end{tabular}




\begin{tabular}{|l|l|l|}
\hline Lysine & Glycation \\
\hline Lysine & $\begin{array}{l}\text { Ubiquitin-activating } \\
\text { enzymes, Ubiquitin ligases }\end{array}$ & Sumoylation \\
\hline Asparagine & $\begin{array}{l}\text { Glycosyltransferases, } \\
\text { deglycosylases }\end{array}$ & Glycosylation N-linked \\
\hline Serine/ Threonine & $\begin{array}{l}\text { Glycosyltransferases, } \\
\text { deglycosylases }\end{array}$ & Glycosylation O-linked \\
\hline Glycine & $\begin{array}{l}\text { N-Myristoyltransferases } \\
\text { Arginine, Threonine }\end{array}$ & Myristoylation \\
\hline Tyrosine & $\begin{array}{l}\text { Sulfatases, sulfatases } \\
\text { protein }\end{array}$ & Sulfation \\
\hline Cysteine & Prenylation/Paltranaferases, & \\
\hline
\end{tabular}


\begin{tabular}{|l|l|l|}
\hline Cysteine, Methionine & & S-nitrosylation \\
\hline
\end{tabular}

Post-translational modification alter protein's structure and also affects its cellular and physiological functions. This leads to change in protein's size, charge, or conformation, altering its hydrophobicity, binding affinity and enzyme activity. Some of the common posttranslational modifications found in eukaryotes along with their target amino acids and enzymes involved are highlighted in Table 1. Posttranslational modifications of the proteins can be detected by various methods. The analytical methods like column chromatography, thin-layer chromatography, radiolabeling of proteins and polyacrylamide gel electrophoresis is used for the detection of PTM. Apart from that protein sequencing and Western blot is also widely used. Currently, mass spectrometry and antibody based detection is most commonly used. Antibody- based detection is based on the availability of the polyclonal or monoclonal antibodies that can specifically recognize the modified amino residue present in the peptide or the protein. This is accompanied by the detection either by using the Chemiluminescence (Western blotting) or the Absorbance/Fluorescence (ELISA) methods. On the other hand, detection of PTM by mass spectrometry is based on the change in mass. Based on the type of the modification involved there occurs the specific change in the mass of the peptide/ amino acid which is confirmed by the Mass spectrometry. Tandem mass spectrometry is also used to identify the modification involved at the specific site ${ }^{1-13}$.

Among the various PTM, phosphorylation is the most common modification which involves the reversible addition of the phosphoryl group to the three amino acids: serine, 
threonine or tyrosine from the adenosine triphosphate (ATP). This modification leads to change in conformation resulting into altered cellular function and signaling. The changes involve activation or deactivation of an enzyme by affecting its catalytic activity and/or increase a tendency of a protein to misfold and aggregate. Secondly, phosphorylation also helps to bind other proteins by facilitating its recruitment. Likewise, considering acetylation, about $80-90 \%$ of the eukaryotic proteins are acetylated. However, it is not clear what the biological role of those acetylated proteins are. Acetylation leads to blockage of positively charged lysine side chains by the neutral acetyl groups thereby altering the protein's charge distribution. Though, acetylation was first reported in histones, it is also observed in cytoplasmic proteins. Lysine acetylation regulates gene transcription in histone proteins. Histone acetylation is related to transcriptional activation whereas transcriptio nal suppression is the result of deacetylation. Talking about ubiquitination, it involves addition of $8 \mathrm{kDa}$ polypeptide to the target proteins through the $\mathrm{C}$-terminal glycine present in ubiquitin. Ubiquitination also targets lysine residues like acetylation and methylation. Ubiquitination acts as a signaling scaffold during biological responses. The proteins that are targeted by ubiquitination are usually the ones that needs to relocated or degraded.

Posttranslational modification plays important role in protein folding and aggregation. Among these modifications like phosphorylation, acetylation, ubiquitination are observed in many diseases including neurological disorders. For example: In Parkinson's disease, which affects around 6.3 million worldwide, $\alpha$-synuclein protein is involved in this disease and posttranslational modifications like phosphorylation, ubiquitination or nitration are known to be involved in the aggregation of $\alpha$-synuclein protein and is known to possess several impacts in its cellular neurotoxicity. Due to the role of PTMS, in a signaling 
pathways, in many diseases they are used as a potential therapeutic target or as a biomarker. Studying PTMs also helps to understand the disease pathology.

\subsection{Protein aggregation}

The key macromolecules of the living systems include proteins, lipids, carbohydrates and nucleic acids. Among which protein plays a vital role in performing wide variety of functions. About 100,000 types of proteins are involved in controlling the reactions involved in life of the living organisms. How one protein is distinguished from another is based on the constituents of amino acids ${ }^{17}$.

Failure of a polypeptide chain to maintain or reach its three-dimensional structure leads to aggregation. Though protein aggregation is widely studied topic but there are some key questions which some unanswered questions which includes: Mechanism underlying the protein aggregation, nature and structure of the aggregates, reason behind same protein leading to ordered and disordered aggregates, toxic species, specificity of intermolecular interactions, effect of environmental factors on aggregation rate and methods to prevent those aggregation ${ }^{18}$. In most of the cases, aggregation is usually irreversible which is due to the equilibrium shifted towards favor of aggregate rather than soluble form. Some of the aggregates like in vivo amylo id deposits are reversible ${ }^{19,20}$. However, under the native like conditions the process is irreversible once the insoluble aggregates are formed.

Protein misfolding diseases includes a broad range of diseases which occurs due to failure of a specific protein /peptide to maintain its native conformational state. Many of these misfolding diseases are associated with the formation of organized fibrillary structures rich 
in $\beta$-sheet structures from its soluble form ${ }^{21,22}$. Although the work by Christian Anfinsen showed that the proper folding of protein is directed by the amino acid sequence of it, now it is acknowledged that the variations in the folding trajectory of a protein to its native form including alternative states associated to pathogenesis is also governed by the same sequence. Apart from these different environmental as well as metabolic stresses, molecular crowding, aging, synthesis errors that are related to the cellular environment also act in addition to sequence-based challenge.

\subsubsection{Types of protein aggregates}

Basically, there are two broad classification of protein aggregates. Protein aggregates are classified as in vitro and in vivo aggregates, fibrillar and amorphous aggregates. Apart from these, aggregates are also classified as physical and chemical, reversible and irreversible, soluble and insoluble. The amyloid fibrils are the ordered aggregates that are observed both in vivo and in vitro whereas the disordered aggregates are the inclusion bodies that are amorphous and are formed in vivo. The aggregates that are formed in vitro during refolding of the proteins at high concentration are usually disordered aggregates. The physical aggregates refers to the non-covalent and chemical aggregates refers to the covalent aggregates. Similarly, the soluble oligomers include dimer to decamer ${ }^{22}$.

\section{Amyloid fibrils:}

The term amyloid refers to extracellular proteinaceous deposits which are abnormal fibrous and are found in tissues and organs. Initially, the word was coined by Schleiden and in mid-19 $19^{\text {th }}$ century Virchow described the term in the deposits of iodine stained observed in 
the autopsy of liver. Amyloid fibrils are formed from soluble proteins which turn into insoluble structure rich in $\beta$-sheet structure and are resistant to degradation. Later on, the properties of the amyloid like specifically binding to the Congo red was discovered suggesting the structure of amyloid to be fibrillar. The use of transmission electron microscopy and X-ray fiber diffraction pattern further confirmed the morphology and cross- $\beta$ structure of amyloid. Ranging from size of $7-12 \mathrm{~nm}$ in diameter, amyloid fibrils are dissociated at high concentration of denaturant. Compared to other fibrous protein, the role of amyloids in providing structural, motility or support is not known but is related to number of the diseases which are known as amyloidosis. Some of these diseases includes Alzheimer's, type II diabetes, the spongiform encephalopathies ${ }^{23}$. Understanding the formation and physicochemical properties of amylo id is the highly studied area.
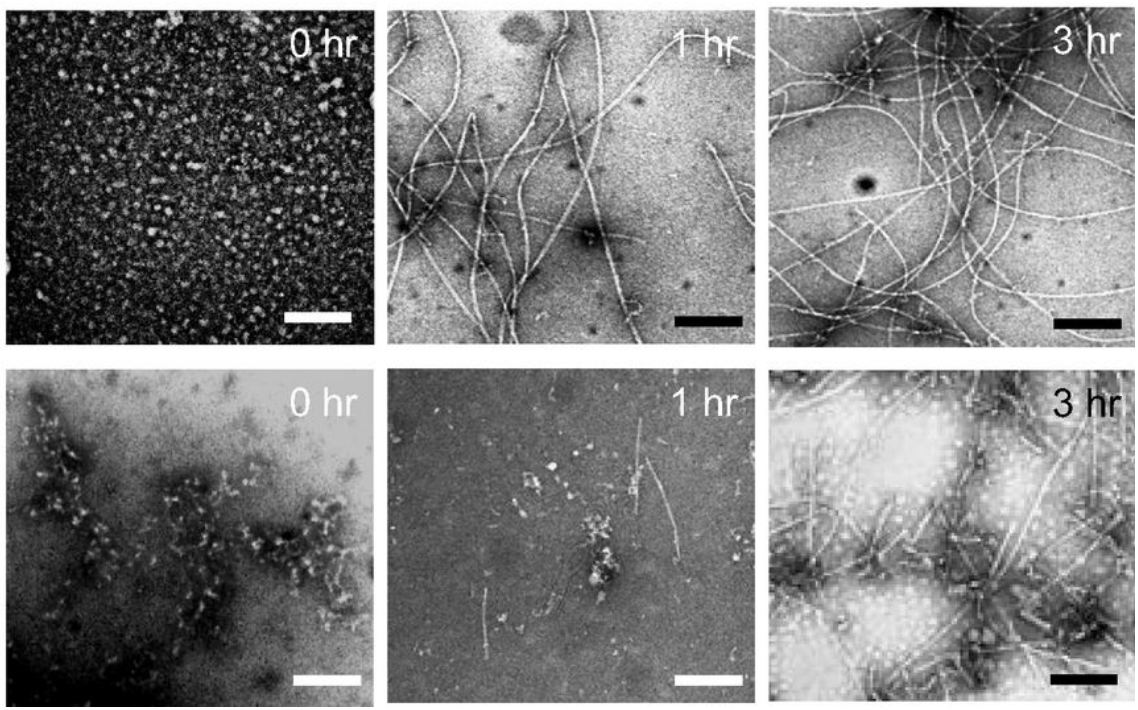

Figure 3: TEM images of $\mathrm{A} \beta_{1-42}$ aggregates in LMW (top) or HMW (bottom) incubation at the indicated times ${ }^{24}$. (Scale bars, $100 \mathrm{~nm}$.). Reprinted with permission from Nakayama, W.T. et.al. $(2016)^{24}$. 


\section{Amorphous aggregates}

Amorphous aggregates are the disordered structures and are common to most proteins. Formation of amorphous aggregates are observed during the process of protein expression purification and even when amyloidogenic proteins fails to form amyloid fibrils. Evidence suggests that amorphous aggregates are also observed in disease conditions ${ }^{25,26}$.

Protein aggregation and understanding the mechanism underlying it is important to researchers in academia as well as industry. Amyloidogenic aggregates are hallmark of several diseases such as type II diabetes, systemic amyloidosis, Alzheimer's, and Parkinson's diseases. However, role of amorphous aggregation in the disease process is not understood very well and were thought not to be associated with any diseases. Nevertheless, recent research shows that it is found in several diseases. For instance: Cause of cataracts is due to aggregation of crystalline protein which are amorphous in nature ${ }^{27}$. In Parkinson's disease, in addition to an amyloidogenic structures, formation of amorphous aggregates by $\alpha$-synuclein have been reported ${ }^{28}$. Also, in TDP-43 protein associated aggregates, both amorphous and amyloid fibrils have been reported by different groups. Formation of amorphous aggregates is of greatest importance especially in the production as well as storage of industrial enzymes and therapeutic proteins ${ }^{29}$. Although importance of amorphous aggregates in the disease process has been established, it is not clear which form of aggregate is more toxic. Protein aggregates size can be characterized by different methods like Light Scattering method (LS), Dynamic Light Scattering (DLS), and Size Exclusion Chromatography (SEC). 

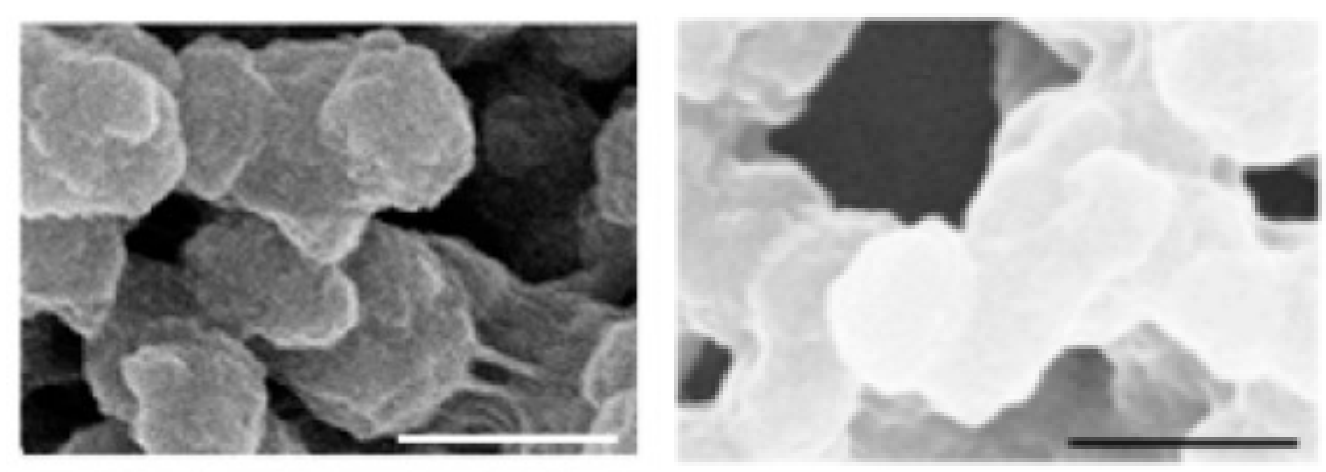

Figure 4: SEM images of lysozyme insoluble aggregates. The insoluble aggregates of lysozyme in presence of $10 \mathrm{mM}$ DTT incubated for $4 \mathrm{~h}$ (left) and $7 \mathrm{~d}$ (right) respectively. Scale bar $=1 \mu \mathrm{m}$ for both images. Reprinted with permission from Yang et.al. $(2015)^{30}$ Copyright (C) 2015 American Chemical Society.

The nature or morphology of the protein aggregates is governed by several factors. It is not characterized by the sequence of amino acids alone as the same protein can form amorphous or fibrillar structure depending on the environmental conditions it is exposed to. Among these factors like $\mathrm{pH}$, temperature, external environment, salt concentration etc., $\mathrm{pH}$ is known to play a key role in determining the nature of aggregates. This is because $\mathrm{pH}$ affects the net charge on the proteins. It has been reported that the proteins preferably form fibrillar structure when they have high net charge and when the $\mathrm{pH}$ favors low net charge the result is amorphous like aggregates. This finding has been supported by a study done on seven different proteins where effect of $\mathrm{pH}$ resulted different morphology of a protein .

Protein aggregation and toxicity is widely studied topic. Also, there has always been a question regarding the nature of aggregates and its associated toxicity, i.e. which aggregate form of the protein is most toxic. Until recently, the mature amyloid fibrils that are mostly 
detected in the pathological diseases were considered to be most toxic to the cells. A number of experimental data now suggests that pre-fibrillar aggregates, which are close in properties to amorphous aggregates, protofibrils or protein micelles are most-toxic to cells. This has been reported in number of studies carried out with $\mathrm{A} \beta$ peptides, $\alpha$-synucle in indicating that the toxic species are the early aggregates. This suggests importance of understanding the toxic nature of aggregates as all aggregates generated and rich in $\beta$-sheet are not necessarily toxic in nature.

\subsection{Protein aggregation in neurodegenerative diseases}

Accumulation of protein aggregates in the neurons is a common feature observed in several neurological disorders. Neurodegenerative disorders are a clinical condition that involves the loss of neurons gradually leading to the cognitive, behavioral and physical defects ${ }^{31}$. In order to identify the neurological pathologies, the identification of protein involved in neurodegenerative disease is essential ${ }^{31}$. There are several neurodegenerative diseases like Alzheimer's disease (AD), Parkinson's disease (PD), Huntington's disease (HD), Amyotrophic lateral sclerosis (ALS), Lewy Body dementia, and Prion like disease identified, and each disease has a unique protein and gene involved in it. Involvement of different proteins leading to distinct clinical symptoms and diseases have complicated the research focusing on identifying some major common properties shared by these disease proteins.

Amyotrophic Lateral Sclerosis (ALS) is a neurodegenerative disease that causes axonal degeneration leading to motor neuron dysfunction and cell death. ALS is a sporadic disease but about $10 \%$ of the cases are found to be a familia ${ }^{33}$. Copper /Zinc Superoxide dismutase 
1 (SOD1) is the major enzyme involved in ALS and mutations in SOD1 has resulted in major progress in the identification of pathogenesis in the field of ALS. The pathogenesis of SOD1 mutant is associated with the gain of toxic function and not with the loss of normal function.

Among the various proteins identified in neurodegenerative diseases, Trans-active response (TAR) DNA binding protein 43 (TDP-43) is increasingly implicated in several neurodegenerative diseases like Alzheimer's disease, ALS, FTLD-U, Huntington and Lewy body diseases. This explains TDP-43 as a high value target protein. Knowing the involvement of a common protein in multiple neurodegenerative diseases has a greater application in identifying the mechanism involved in all these disease conditions. In case of neurodegenerative disorders, the common features seem to be the presence of insoluble intracellular aggregates, that are often phosphorylated and ubiquitinated ${ }^{34-36}$. Association of TDP-43 is found to be in neurodegenerative diseases as the aggregates of this protein are present in patients with ALS and FTLD. In cases of TDP-43, the pathology has distinguishing features like mislocalization of TDP-43 to the cytoplasm, the loss of nuclear localization, as well as truncation. Therefore, TDP-43 proteinopathies involves all the characteristic features that are linked to neurodegenerative diseases having the TDP-43 pathology ${ }^{34}$. 


\subsection{Proteins used in study}

\subsubsection{TDP-43}

Trans-active response (TAR) DNA binding protein is a $43 \mathrm{kDa}$ protein, first identified in 1995 as transcriptional inactivators of HIV virus ${ }^{35-37}$. This protein could bind with the TAR DNA element of HIV virus, hence named TAR DNA binding protein. TDP-43 is a 414 amino-acid protein belonging to the family of heterogeneous nuclear ribonucleoproteins and share many features of this family ${ }^{35,38}$. TDP-43 is highly conserved among invertebrates and mammals, with its expression present in nearly all tissues ${ }^{39}$. Structurally, distinguished by two RNA recognition motifs (RRM) i.e. RRM1 and RRM2 along with Nterminal and C-terminal regions, this protein is best known as RNA and DNA binding protein $34-35,40-43$. TDP-43, being a protein of nuclear origin is known to play various roles in metabolism of RNA including mRNA stability, splicing, transport, miRNA processing, transcriptional repression. Under the pathological conditions these functions are altered or there is an abnormal function leading to neurotoxicity. Moreover, the actual cause of neurotoxicity is still not clear. Some studies support toxicity due to gain of function and others support the toxicity due to loss of functions First identified in 2006, in a ubiquitinated aggregates of a FTLD and ALS associated patients, TDP-43 protein caught attention of many researchers and since then about 18,200 papers have been published on TDP-43 till date. Accumulation of TDP-43 aggregates is present in approximately $98 \%$ ALS and 50\% FTD. Apart from that 50\% of Alzheimer's disease is associated with TDP43 pathology. 


\subsubsection{Amyloid-Beta}

Alzheimer's disease (AD) is a progressive neurodegenerative disorder and distinct $\mathrm{A} \beta$ aggregates are observed as the disease progresses. Getting back into 1907, Alois Alzheimer first reported the findings of an autopsy of 55-year women as presence of neurofibrillary tangles and neuritic plaques who died of progressive behavioral and cognitive disorder. In 1984, Glenner and Wong purified and found that these substances was a peptide of 4.2$\mathrm{kDa}$, with amino acids of 40 or 42 in length, which was thought to be cleaved from some large precursor. In 1987, Kang et.al. cloned the amyloid precursor protein which confirmed the prediction made by Glenner and Wong and the peptide isolated was known as Amyloid $\beta$ peptide in short $\mathrm{A} \beta$ peptide p $^{4}$.

Amyloid beta is produced as a proteolytic product of the precursor protein known as APP which results from the cleavage by proteases, $\beta$-and $\gamma$-secretases. The proteolytic processing of APP results into two products of varying lengths especially at the carboxy terminal of the peptide. The two different forms of $\mathrm{A} \beta$ are of 40 and 42 amino acids in length known as $A \beta 40$ and $A \beta 42$ respectively. In normal individual, $A \beta 40$ is the major form of $A \beta$ produced. In a pool of $A \beta$ only $5-15 \%$ of total $A \beta$ is occupied by $A \beta 42^{45}$. Though $A \beta 40$ is present in higher concentration in cerebrospinal fluid compared to $A \beta 42$, the amyloid plaques from $\mathrm{A} \beta 42$ comprises the major component in $\mathrm{AD}$ brains. Further, Ratio of $\mathrm{A} \beta 42$ / $\mathrm{A} \beta 40$ plays a critical role in $\mathrm{AD}$, where the increased ratio is associated with the early onset of familial $\mathrm{AD}$ and lowered ratio leads to decreased deposition of $\mathrm{A} \beta$ in transgenic mice. Also, the higher ratio correlates with the increased neurotoxicity. A $\beta 42$ is more aggregation prone compared to $A \beta 40$, hence results into early deposition than 
$A \beta 40$. This leads to explanation that although both isoforms are present, $A \beta 42$ and $A \beta 40$ preferentially aggregates with their own species.

\subsection{Motivation:}

Protein misfolding and aggregation is at the core of many neurodegenerative diseases. The common pathophysiological feature shared for several neurodegenerative diseases is accumulation of distinctly misfolded and aggregated proteins in brains, in cytosol, intranuclear, or extracellular spaces. Different neurodegenerative diseases like Amyotrophic Lateral Sclerosis, Frontotemporal dementia, Alzheimer's disease, Parkinson's diseases, Huntington diseases, Lewy body diseases etc. have characterized distinct aggregates in distinct part of the neuron. The unique characteristic is that each of these diseases has specific protein involved in its pathogenesis, e.g. SOD1 in ALS, Amyloid $\beta$ in Alzheimer's, and $\alpha$-synuclein in Parkinson's disease. While the general mechanism of toxicity in these diseases in understood, still the molecular level of understanding for these diseases is a subject of intense debate. This is complicated further by lack of information on distinct physicochemical properties of proteins including its aggregated structures at a molecular level, making difficult to understand its role in the disease processes.

The main focus of this research is expression and purification of full- length TDP-43 protein using eukaryotic expression system and understand its aggregation property using reducing and non-reducing environment and test its toxicity which is covered in Chapter 3. A number of studies have been carried out in TDP- 43 protein using the prokaryotic expression system. However, having method for purification in eukaryotic system is 
lacking. Therefore, in this research we have explained the standardized methods for purification and addressed some of the aggregation and toxicity properties of full length aggregates. This will help to shed some light in the understanding the role of the protein in the disease process.

Posttranslational modification is the common modifications that takes place in protein. Among the various modifications, acetylation of protein is one of the major modification that taking place in eukaryotes. $A \beta$ is the protein which is involved in Alzheimer's disease. Study on the effect of the lysine acetylation on this protein is not reported in detail, though information of acetylation on lysine 28 is available. Therefore, in chapter 4 , the effect of acetylation on lysine16 on $\mathrm{A} \beta$ (1-42) aggregation is discussed. For this purpose, the single acetylated lysine residues at position 16,28 or double acetylated KKAc was used and its comparison with WT was performed. Understanding the effect of lysine acetylation on A $\beta$ (1-42) aggregation will help to develop the proper therapeutic targets for treatment of Alzheimer's disease.

As discussed in above sections, several factors like $\mathrm{pH}$, temperature, reducing environment also causes protein destabilization that leads to protein aggregation. Therefore, understanding the role of protein either by changing the charge on the protein, or by reducing the disulfide bonds in it helps in providing some clue on its pathogenesis. As evident, same protein can generate different types of aggregates under the conditions it is exposed, it is equally important to understand under what conditions the different aggregates are formed and what is the level of toxicity of those aggregates at cellular level. Given that, not all aggregates generated are toxic, if we could investigate the toxicity of 
aggregates based on its morphology or biophysical properties would provide a big insight in understanding the molecular mechanism in disease process as well as help in selecting the targets for drug discoveries. This topics will also be discussed in chapter 3 and 4 .

Explanation of techniques used in conducting experiments described in chapters 3, 4 will be discussed in Chapter 2. Various techniques ranging from cell biology, biophysical and biochemical and imaging will be covered. This provides details on each technique along with its applications which helps to guide as we go through experimental details in Chapter 3 and 4.

Future applications of the projects are discussed in Chapter 5. In future, since we have already standardized the method of purification of full-length protein, more TDP-43 fulllength protein can be generated to study in great detail of its biophysical and biochemical properties. Though, we have some preliminary data on disulfide status of protein by treating with mal-PEG, more extensive study can be conducted using different concentration of reducing agent and mal-PEG. This will help us to understand the disulfide status of the protein which is still ambiguous to all researchers. In addition, the role of $\mathrm{pH}$ on protein stability, structure and toxicity can be studied by altering the $\mathrm{pH}$ of the protein and observing its aggregation property, morphology and toxicity. Not only that the protein's biophysical properties like hydrophobicity, flexibility etc. can be studied in great detail using biophysical techniques. Gathering all these, information's together will provide some useful answers on structure of this protein and its associated toxicity. 


\subsection{References:}

1. Hart, F.U.; Bracher, A.; Hart, M.H., Molecular chaperones in protein folding and proteostasis. Nature Review 2011, 10, 324-332.

2. Forman, M.S.; Trojanowski, J.Q.; Lee, V.M., Neurodegenerative diseases: a decade of discoveries paves the way for therapeutic breakthroughs. Nature Medicine 2004, 10: 1038/1113. 3 .

3. Soto, C., Unfolding the roles of protein misfolding in Neurodegenerative Diseases. Nature Reviews 2003, 4:49-60.

4. Nick, P.C. et.al, Forces Stabilizing Proteins. FEBS Lett. 2014, 588(14): 2177-2184. doi: 10.1016/j.febslet.2014.05.006.

5. Dill, K.A., Dominant Forces in Protein folding. Biochemistry 1990, 29(31):7133-7155.

6. Fersht, A. R., Conformational equilibria in -and -chymotrypsin. The energetics and importance of the salt bridge. Journal of molecular biology 1972, 64, 497-509.

7. Perutz, M. F.; Raidt, H., Stereochemical basis of heat-stability in bacterial ferredoxins and in hemoglobin-A2. Nature 1975, 255, 256-9.

8. Grishman, G., Biochemistry , third edition, ISBN: 2006935521

9. Englander, S.W., The nature of protein folding pathways. Proc Natl Acad Sci U S A. 2014, 111(45): 15873-15880.

10. Nelson, L.D., Lehninger, Fourth Edition, ISBN: 1-4039-4876-3, pages: 148-149. 
11. Ana, L. S.; Ariel, B.L., Protein Posttranslational Modifications: Roles in Aging and Age-Related Disease. Oxi Med cell Longev. 2017: 5716409.

12. Greifenhagen, U.; Frolov, A.; Blüher, M.; Hoffmann, R., Site-specific analysis of advanced glycation end products in plasma proteins of type 2 diabetes mellitus patients . Analytical and Bioanalytical Chemistry 2016, 408(20):5557-5566. doi: 10.1007/s00216016-9651-4.

13. Nedić, O.; Rogowska,W.A.; Rattan S. I. S., Standardization and quality control in quantifying non-enzymatic oxidative protein modifications in relation to ageing and disease: why is it important and why is it hard? Redox Biology. 2015, 5:91-100. doi: 10.1016/j.redox.2015.04.001.

14. Kakizawa, S., Nitric oxide-induced calcium release: activation of type 1 ryanodine receptor, a calcium release channel, through non-enzymatic post-translational modification by nitric oxide. Frontiers in Experimental Endocrinology 2013, 4: p. 142. doi: 10.3389/fendo.2013.00142.

15. Merkley, E. D.; Metz, T. O.; Smith, R. D.; Baynes, J. W.; Frizzell, N., The succinated proteome. Mass Spectrometry Reviews 2014, 33(2):98-109. doi: 10.1002/mas.21382.

16. Kuhn, M. L.; Zemaitaitis, B.; Hu, L. I. et al, Structural, kinetic and proteomic characterization of acetyl phosphate-dependent bacterial protein acetylation. PLoS One. 2014, 9(4) doi: 10.1371/journal.pone.0094816. 
17. Chaturvedi, S.K., Protein misfolding and aggregation: Mechanism, factors and detection. Process Biochemistry 2016, 51 (9)1183-1192.

18. Fink, A.L., Protein aggregation: folding aggregates, inclusion bodies and amyloid. Folding and Design 1998, 3(1): R9-R23.

19. Deyoung, L.R.; Dill, K.A.; Fink, A.L., Aggregation and denaturation of apomyoglob in in aqueous urea solutions. Biochemistry 1993, 32, 3877-3886.

20. Tennent, G.A.; Lovat, L.B.; Pepys, M.B., Serum amyloid P component prevents proteolysis of the amyloid fibrils of Alzheimer disease and systemic amyloidosis. Proc. Natl Acad. Sci. USA 1995, 92, 4299-4303.

21. Holmes, W.L. et.al., (2014) Defining the limits: Protein aggregation and toxicity in vivo. Critical Reviews in Biochemistry and Molecular Biology 2014, 49(4), 294-303.

22. Proteins, Amyloid, and Disease 335 Annu. Rev. Biochem. 2006.75:333-366. Downloaded from www.annualreviews.org Access provided by Michigan Technological University - J. R. VAN

23. Yuichi, Yoshimura. et.al, Distinguishing crystal-like amyloid fibrils and glass-like amorphous aggregates from their kinetics of formation. PNAS 2012, 109 (36) 1444614451.

24. Watanabe, N.T.; Ono, K.; Itami, M.; Takahashi, R.; Teplow, D.B.; Yamada, M., Highspeed atomic force microscopy reveals structural dynamics of amyloid $\beta 1-42$ aggregates. Proc Natl Acad Sci U S A. 2016, 113(21),5835-40. 
25. Sugiyama, M. et al., SAXS and SANS observations of abnormal aggregation of human $\alpha$-crystallin. Chem Biodiversity 2010, 7,1380-1388.

26. Truscott, R.J.; Age-related nuclear cataract-oxidation is the key. Exp Eye Res 2005 80:709-725.

27. Dee, Groot N.S.; Sabate, R.; Ventura, S.; Amyloids in bacterial inclusion bodies. Trends Biochem Sci 2009, 34,408-416.

28. Wang, L.; Maji, S.K.; Sawaya, M.R.; Eisenberg, D.; Riek, R., Bacterial inclusion bodies contain amyloid-like structure. PLoS Biol 2008, 6:1791-1801.

29. Agostini, F.; Vendruscolo, M.; Tartaglia, G.G., Sequence-based prediction of protein solubility. J Mol Biol 421:237-241. 10. Stranks SD, et al. (2009) Model for amorphous aggregation processes. Phys Rev E 2012, 80:051907.

30. Yang, M.; Dutta, C.; Tiwari, A., Disulfide-Bond Scrambling Promotes Amorphous Aggregates in Lysozyme and Bovine Serum Albumin. Journal of Physical Chemistry B 2015, 119, 3969-81.

31. Hirth, F., Drosophila melanogaster in the Study of Human Neurodegeneration. CNS \& neurological Disorders -Drug Target 2010, 9, 504-523.

32. Romano, V.; Quadri, Z.; Baralle, F.E.; Buratti, E., The structural integrity of TDP-43 $\mathrm{N}$-terminus is required for efficient aggregate entrapment and consequent loss of protein function. Prion 2015, 9,1-9. 
33. Banci, L. et.al., SOD1 and Amyotrophic Lateral Sclerosis: Mutations and Oligomerization. PLoS One 2008, 3(2), e1677.

34. Lee, B.E.; Lee, V.M.Y.; Trojanowski, J.Q., Gains or losses: molecular mechanisms of TDP-43- mediated neurodegeneration. Nature reviews 2012, 13, 38-48.

35. Ayala, V.; Serrano, A.BG. et.al., Cell stress induces TD-43 pathological changes associated with ERK1/2 dysfunction: implication in ALS. Acta Neuropathol 2011, 122:259-270.

36. Xu, S.Z., Does a loss of TDP-43 function cause neurodegeneration? Molecular Neurodegeneration 2012, 7(27):1-10.

37. Buratti, E.; Baralle F. E., Multiple roles of TDP-43 in gene expression, splicing regulation, and human disease. Frontiers in Bioscience 2008, 13, 867-878.

38. Hanson, K.A.; Kim, S.H.; Tibbetts, S.R., RNA- binding proteins in neurodegenerative diseases: TDP-43 and beyond. WIREs RNA 2012, 3, 265-285.

39. Rohn, T.T., Cytoplasmic inclusions of TDP-43 in neurodegenerative diseases: A potential role for Caspases. Histol Histopathol. 2009, 24, 1081-1086.

40. Huang, Y.C.; Lin, K.F.; He, R.Y.; Tu, H.P.; Koubek, J.; Hsu, Y.C.; Huang, J.J., Inhibition of TDP-43 aggregation by Nucleic Acid Binding 2013, PLOS ONE 8(5): 1-11. 
41. Cohen, T.J.; Hwang, A.W.; Unger, T.; Trojanowski, J.Q.; Lee, V M.Y. Redox signaling directly regulates TDP-43 via cysteine oxidation and disulphide cross-linking. The EMBO Journal 2012, 41, 1241-1252.

42. Tourenne, C.L.; Polymenidou, M.; Cleveland, D.W., TDP-43 and FUS/TLS: emerging roles of RNA processing and neurodegeneration. Human Molecular Genetics 2010,19(1), R46-R64.

43. Buratti, E.; Brindisi, A.; Pagani, F.; Baralle, F.E., Nuclear factor TDP-43 binds to the polymorphic TG repeats in CFTR intron 8 and causes skipping of exon 9: a functional link with disease penetrance. Am J Hum genet 2004, 74, 1322-1325.

44. O'Brien, R., Amyloid Precursor Protein Processing and Alzheimer's Disease. Annu Rev Neuroscience 2011,34,185-204.

45. Guo, L.GZ., Alzheimer's $A \beta 42$ and $A \beta 40$ peptides form interlaced amyloid fibrils. Journal of Neurochemistry2013, 126(3), 305-311. 


\section{Chapter 2: Methods}

In this chapter, I will discuss about the different techniques used in accomplishing the work explained in Chapter 3 and 4. This chapter serves as providing detailed information's on each techniques with its applications and limitations. The work in one of the chapter is about the expression and purification of TDP-43 protein. And I also carried out biophysical and biochemical characterization of protein. To better understand the methods involved I have represented each project in flow-diagram along with its description.

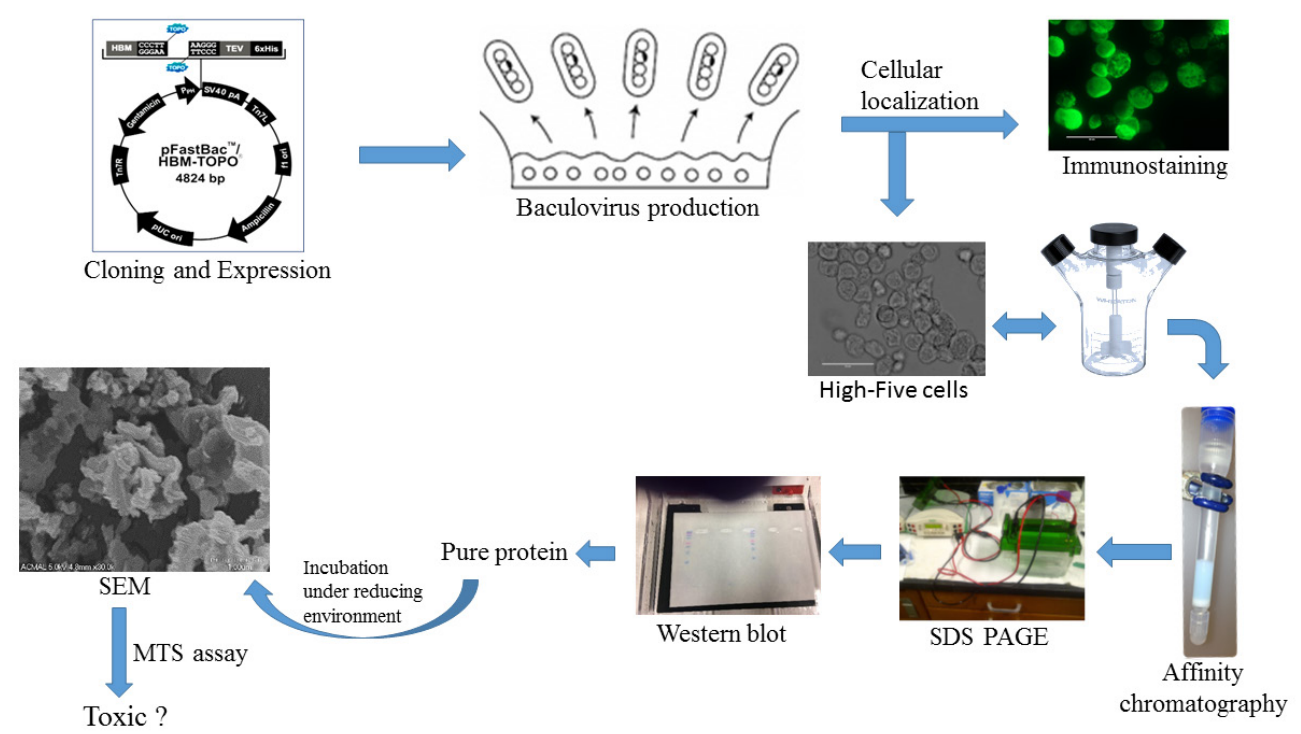

Figure 1: Schematic representation of techniques used in Expression and purification of TDP-43 protein. 


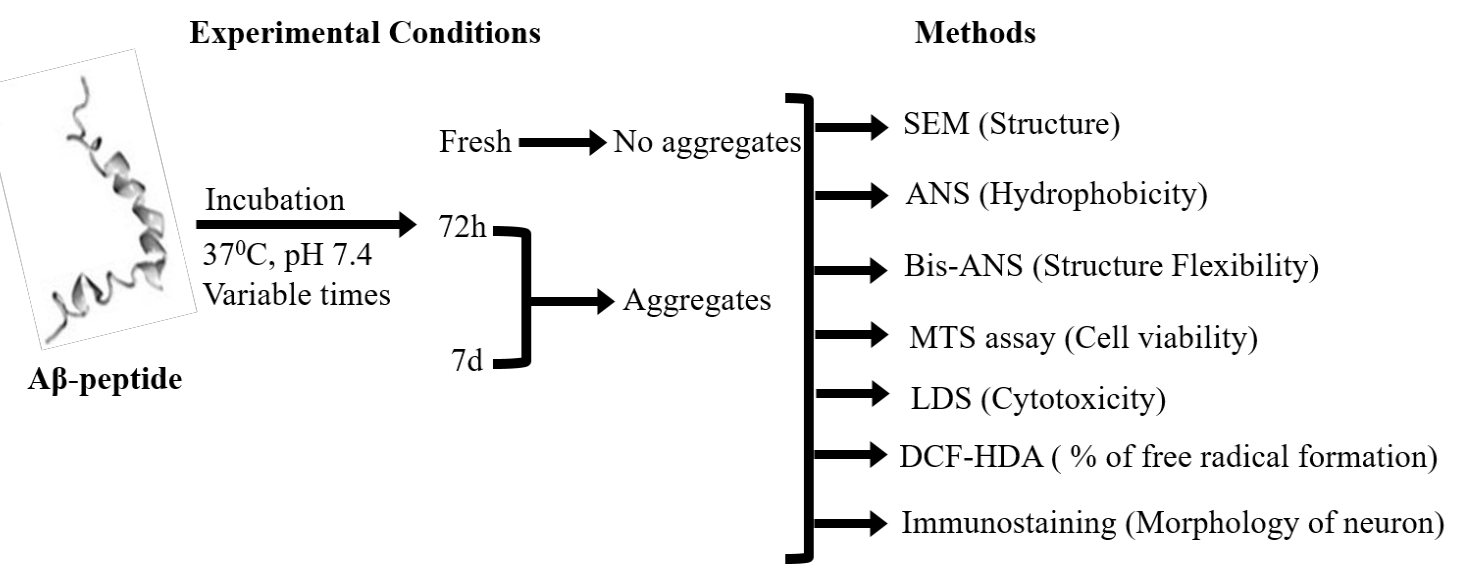

Figure 2. Schematic representation of techniques used in chapter 4.

\subsection{Affinity chromatography}

Expression and purification of protein is routinely employed in biochemical studies. Among the different purification techniques, affinity chromatography is one of the powerful technique which involves the use of affinity tags that fuses with protein of interest. Immobilized metal-affinity chromatography (IMAC) is used for purification of recombinant proteins which contains short affinity tag like polyhistidine residues. Purification using polyhistidine tags has been used successfully in different expression systems like E.coli, mammalian, baculovirus infected insect cells, Saccharomyces cerevisiae. These tags are generally employed on either $\mathrm{N}$ - or $\mathrm{C}$ - terminal of the protein. IMAC chromatography results into high yield of protein purification with up to $95 \%$ of purity. IMAC employs the interaction of the stationary phase which is the immobilized matrix consisting of transition metal ion $\left(\mathrm{Co}^{2+}, \mathrm{Ni}^{2+}, \mathrm{Cu}^{2+}, \mathrm{Zn}^{2+}\right)$ with the side chain of amino acid. Histidine has the high affinity with the metal ions like nickel ion present in the 
column matrix and results into strong interaction due to the formation of the coordination bonds between imidazole ring on the histidine with the transition metal ion. As a result of which, peptides that contain the histidine residues are retained in the column which can be eluted from the column by increasing the concentration of imidazole in the buffer system ${ }^{1}$, 2.

In Chapter 3, we used Ni-NTA affinity chromatography to purify our proteins. The column was was first packed with HisPur ${ }^{\mathrm{TM}}$ Ni-NTA resin. This resin has the capacity of binding upto $60 \mathrm{mg}$ of $6 \mathrm{XHis}$ tag protein per $\mathrm{ml}$ of resin and is applicable under both native and denaturing conditions. After packing the column, the column was equilibrated with equilibration buffer containing $10 \mathrm{mM}$ imidazole. Then, the protein lysate/urea soluble /detergent soluble fraction was loaded on the column and unbound fraction was collected. The column was further washed with wash buffer containing increasing concentration of imidazole to remove unbound fractions. Finally, the protein was eluted from column using the elution buffer with high concentration of imidazole.

\subsection{Polyacrylamide gel electrophoresis (PAGE)}

Electrophoresis is a technique that involves the separation of proteins based on the migration of a charged particles in an electrical field. Electrophoresis of proteins are generally carried out in Polyacrylamide gel which is a cross linked polymer that acts as a molecular sieve and slows the migration of proteins approximately in proportion to their charge to mass ratio. SDS PAGE (Sodium Dodecyl Sulfate Polyacrylamide Gel Electrophoresis) and Native PAGE are the most commonly used gel electrophores is. SDS PAGE employs SDS (sodium dodecyl sulfate), an anionic detergent that denatures 
secondary and non-disulfide-linked tertiary structures of a protein. One SDS molecule binds to every two amino acid residues thus the SDS confers a large net negative charge on the protein molecule that allows the migration of all proteins in the same direction. The proteins migrate through the pores in the gel towards the positive electrode at the bottom. By the end of electrophoresis, the smaller proteins will be closer to the bottom of the gel and the larger proteins will be closer to the origin. After electrophoresis proteins in the gel can be visualized by the use of Coomassie blue dye which binds to the proteins but not to the gel. Selection of proper gel concentration is critical for separation of proteins. Lower percentage gels are better for resolving high molecular weight proteins, while higher percentages are needed to resolve smaller proteins. SDS PAGE has a wide application like separating different proteins based on their molecular weight, monitoring the progress of protein purification, identifying molecular weight of unknown proteins based on the electrophoretic mobility with reference to standard marker, separating the different subunits of a protein.

Native or Non-denaturing PAGE is a type of gel electrophoresis which is carried out in the absence of SDS so that the proteins are not denatured and are in well-folded structure. In this method, proteins are separated based on the protein's charge and their hydrodynamic volume. The mobility of proteins is based on the intrinsic charge on the protein at the $\mathrm{pH}$ of the running buffer. The proteins in the Native PAGE retain their folded conformation so the migration is also affected by the conformation of proteins i.e: higher mobility is seen for more compact conformations and lower for larger structures like oligomers. Native page is used to identify changes in protein migration due to oligomers, unfolded or molten globule or other modified conformation, changes in charge due to chemical modification 
like degradation and aggregation. Another advantage of native gels is that it is possible to recover proteins in their native state after the separation. Therefore, Native PAGE is one of the most powerful technique for studying the composition and structure of native proteins, since both the conformation and biological activity of proteins remain intact during this technique.

The electrophoresis gels are formulated in buffers such that it provides conduction of an electrical current through the matrix. In order to obtain optimal resolution of proteins stacking gel is casted over the top of the resolving gel. Also, the $\mathrm{pH}$ of Tris in stacking gel is 6.8 whereas the $\mathrm{pH}$ of Tris in Resolving gel is 8.8 which allows the proteins in a loaded sample to be concentrated into a tight band during the first few minutes of electrophores is before entering the resolving gel. This process occurs as Glycine ions has isoelectric point near $\mathrm{pH} 6$ so at that $\mathrm{pH}$ glycine has net charge zero so it migrates slower in the gel and chloride ions has negative charge so can migrate faster in gel and proteins remain in between the Glycine and chloride ions forming a layer in the stacking gel. Selection of proper gel concentration is critical for separation of proteins. The gel consists of acrylamide, bisacrylamide initiator, TEMED, denaturant (SDS) and a buffer with an adjusted $\mathrm{pH}$. Acrylamide has a property of auto polymerization and bisacrylamide forms a cross link with acrylamide and thus results into the gel. The concentration used will depend on the size of the proteins to be separated adequately. Ammonium persulfate is a source for free radical that initiate the process of polymerization. TEMED accelerate the rate of formation of free radicals from persulfate which in turn accelerate the polymerization. APS and TEMED should be prepared fresh as APS and TEMED are highly hygroscopic and start to decompose rapidly in presence of water leading to rapid loss of their reactivity. The 
rate of polymerization and the properties of the resulting gel depend on the concentrations of free radicals. Increasing the amount of free radicals results in a decrease in the average polymer chain length, an increase in gel turbidity and a decrease in gel elasticity. Decreasing the amount shows the reverse effect. Glycerol increases the density of the sample, and bromophenol blue which is a tracking dye that has the mobility higher than other analytes and helps to track the progress of electrophoresis ${ }^{3}$.

In Chapter 2, we used SDS-PAGE for testing our proteins as well as for running western blot. The reduced samples were prepared by adding the $5 \% \beta$-mercaptoethanol in sample whereas non-reduced samples were prepared by incubating the samples with Iodoacetamide for 2 hours in dark and the reaction was terminated by adding the sample buffer without reducing agents. Samples were boiled for 5 minutes and samples were loaded on $12 \% / 15 \%$ SDS gels. The gel were run for 3 hours using Tris-Glycine-SDS running buffer (25mM Tris, $192 \mathrm{mM}$ glycine, $0.1 \% \mathrm{SDS}, \mathrm{pH} 8.3)$. The gels after separation were stained with Coomassie blue and after the destaining process the images were captured on Epson Scanner. 


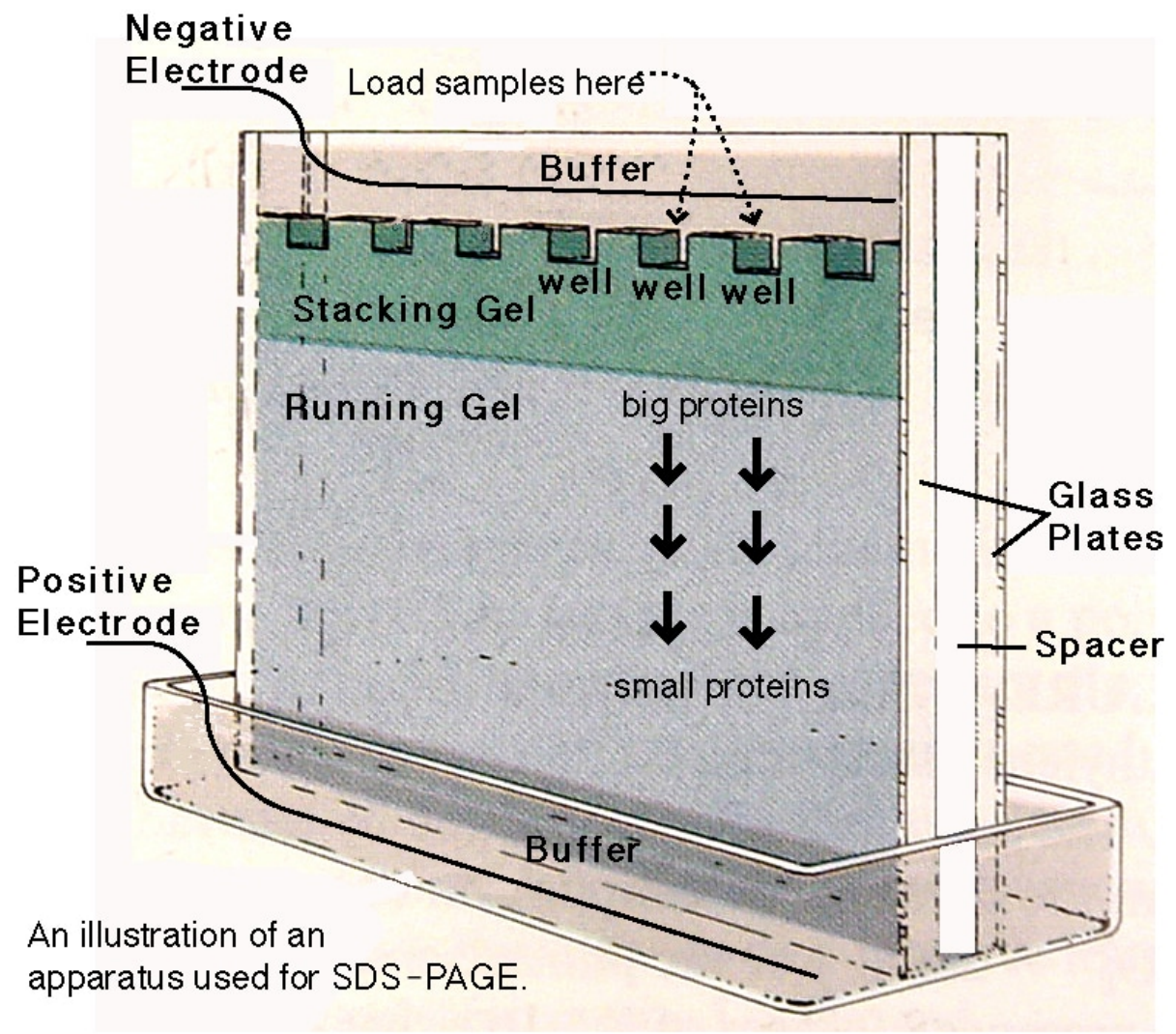

Figure 3: Schematic illustration of SDS-PAGE. Protein samples are loaded on the wells and the samples run from cathode to anode under the application of an electric field. Reprinted with permission of the publisher.

\subsection{Western blot}

Western blot is the most commonly used technique in the cell and molecular biology. It is used to detect the protein in the blot separated by the gel. In this technique, first the protein/mixture of proteins is separated on the basis of molecular weight using the Sodium dodecyl sulfate (SDS) polyacrylamide gel electrophoresis (PAGE). Using a proper membrane which can be PVDF (polyvinylidene difluoride), Nylon, or nitrocellulose, the gel is transferred into the membrane using the transfer buffer under the specific voltage. 
PVDF membrane is more stable compared to other membranes and also allows relabeling and easy storage. PVDF membrane provides high protein binding capacity due to its hydrophobic nature. Nitrocellulose membrane is not good for use of transfer of low molecular weight proteins due to its large pore-size. However, it has the advantages of low background. The membrane becomes brittle and difficult to handle when it's dry. Nylon membrane has lots of advantages but due to its high background binding and irreversible staining of some dyes, it's less common than the other two. The protein band is detected using the antibodies. The membrane after the transfer is blocked with blocking solution which is basically $5 \%$ skimmed milk or $5 \%$ Bovine Serum Albumin. The blocking step is carried out to remove any nonspecific binding. Blocking is followed by the incubation with primary antibody that is specific to the protein of interest. The unbound proteins are washed off and then incubated with the secondary antibody which is HRP conjugated is used to detect the protein band using the chemiluminesence method. The band observed can be quantified that is related with the amount of protein present. Detection method for the western blot can be direct or indirect. In the direct method, only one antibody is required. The primary antibody is conjugated to an enzyme or fluorophore which detects the antigen that is present in the blot. The most commonly employed detection method is the indirect detection method. In this method, the primary antibody is unlabeled, and this is added to blot to bind with the antigen and the detection is facilitated by the secondary antibody that is conjugated to the enzyme or fluorophore which may be biotin, fluorescent probes, HRP or Alkaline phosphatase (ALP). The advantage of using indirect method is same secondary antibody can be used for multiple primary antibodies ${ }^{4}$. 
In chapter 2, we used Western blot to confirm the expression of our protein. After the run of the gels, the gel was transferred into PVDF membrane using the transfer buffer for an hour. The membrane was blocked with 5\% skimmed milk for an hour and then incubated with primary antibody (1:4000) for overnight at $4^{0} \mathrm{C}$. Next day, the membrane was incubated with secondary antibody (1:10000) for 2 hours and finally the image was acquired using the GelDoc.

\subsection{Scanning Electron Microscopy}

Electron microscope uses the electrons instead of visible light used by light microscopes. The electron beams are travelled through the instrument which is facilitated by the high vacuum. Due to the smaller wavelength of the beam the electron microscope is superior to light microscopes in distinguishing smaller details. For example, the wavelength for electrons accelerate by $100 \mathrm{kV}$ is only $0.004 \mathrm{~nm}$ when compared to wavelength of green light which is $550 \mathrm{~nm}$. There are basically two types of electron microscopes: Scanning electron microscopes (SEM) and Transmission electron Microscopes (TEM). Comparing two instruments in terms of resolution, SEM can resolve $1-5 \mathrm{~nm}$ whereas resolution of TEM is $0.1 \mathrm{~nm}$. In TEM, image formation takes place by the electrons beam that is transmitted through the sample, whereas in case of SEM, the beam is scanned across the sample and signals are detected by the detectors which uses the electrons that are reflected from surface

of the sample to generate the image. The field of view is wider in SEM in comparison to TEM. SEM is advantageous over TEM in detection of unstable biomolecules due to its utilization of low voltage in the range of $1-10 \mathrm{kV}$. TEM on the other hand uses high voltage 
in the range of $100-200 \mathrm{kV}$ which makes its use limited to stable biomolecules which is not affected by high voltage.

When the incident electron beam strikes the sample, different types of electron emission are generated depending upon the type of interaction and depth of penetration. These signals can be backscattered electrons, secondary electrons, X-rays, transmitted electrons, scattered electrons, Auger electrons etc. Some of the signals produced are depicted in figure 4.

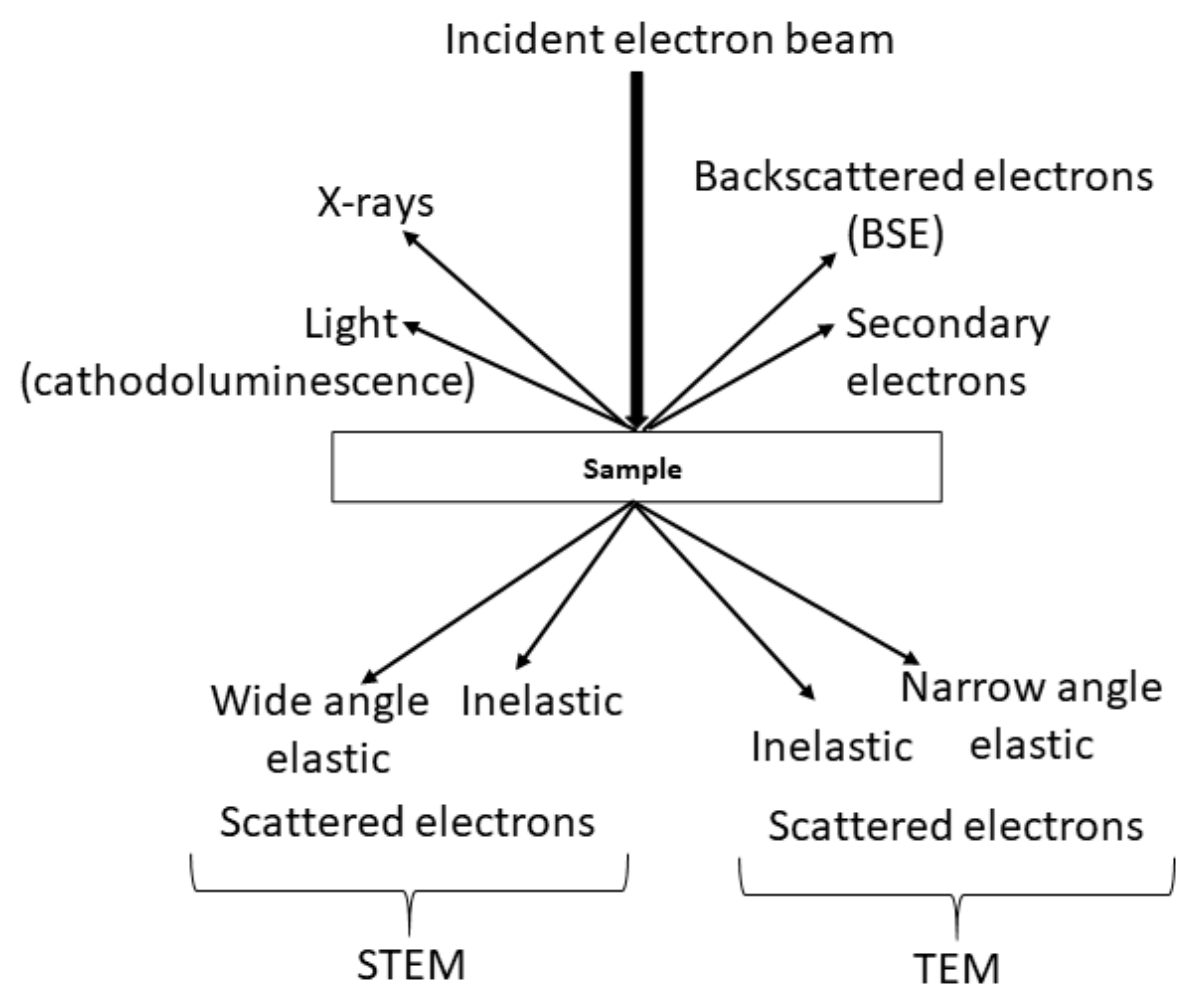

Figure 4: Representation of the different signals that are produced when interaction of an incident electron beam takes place with sample. SEM and TEM are used to detect most of these signals ${ }^{5}$. Figure adapted from 
Scanning Electron Microscopy (SEM) is the most widely used technique for imaging the morphology and composition of various materials. Although the use of SEM was described in 1942, its commercial use became available only in 1965 when Oatley and co-workers from Cambridge University reported their work. SEM has many applications apart from characterizing the topographical, morphological, and compositional information. It is used to detect, analyze or identify the crystalline structures, variations in compositions of chemicals surface fractures analysis, surface contamination examination. In SEM, the images are generated either by the backscattered electron (BSE) or the Secondary electrons (SE). Backscattered electrons are produced when the interaction between beam and the sample is elastic. The specimen's atomic number greatly determines the production of backscattered electrons. As a result of which, the specimen with higher atomic number appears brighter compared to the one with lower atomic number making it's uses in understanding composition of specimen. Secondary electrons are produced when the interaction between beam and the sample is inelastic. SE is the commonly used signal for imaging and is mainly related to the topography and used to detect the morphology of samples 6,7 . 

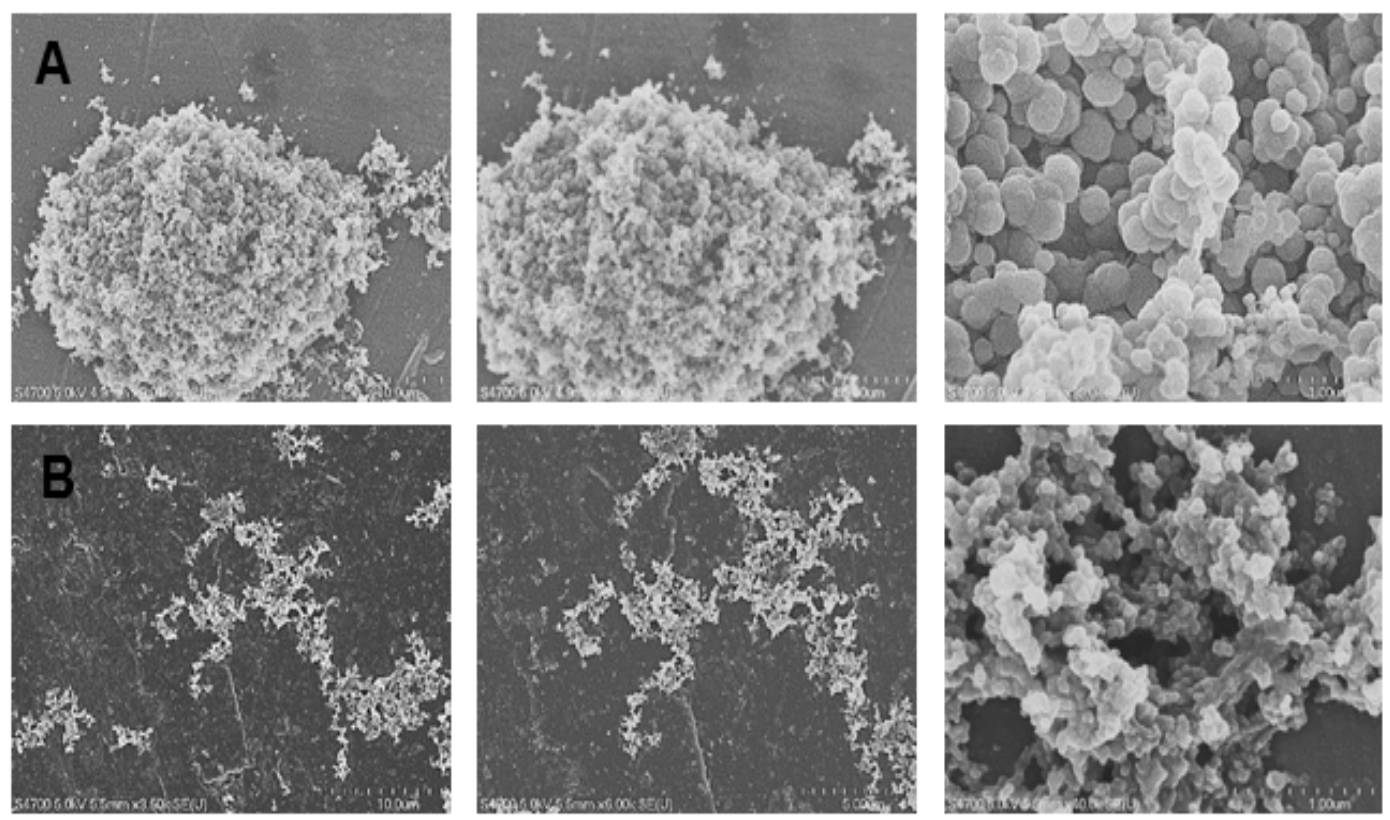

Figure 5: FESEM images of $40 \mu \mathrm{M}$ lysozyme incubated at $37^{0} \mathrm{C}$ for 24 hours. (A) Sample with $1 \mathrm{mM}$ TCEP (B) Sample with $1 \mathrm{mM}$ TCEP and $1 \mathrm{M}$ Glycine. Scale bar $=10 \mu \mathrm{m}, 5 \mu \mathrm{m}$ and $1 \mu \mathrm{m}$ from left to right respectively.

The working principle of SEM is depicted in Figure 6. The electron gun forms a stream of electron under high vacuum. These electrons are then accelerated with upto dozens of Kev energy and focused by the electromagnetic lens into a monochromatic beam. This thin beam inject the sample and the interaction of beam with the sample is detected and transformed into an image. 


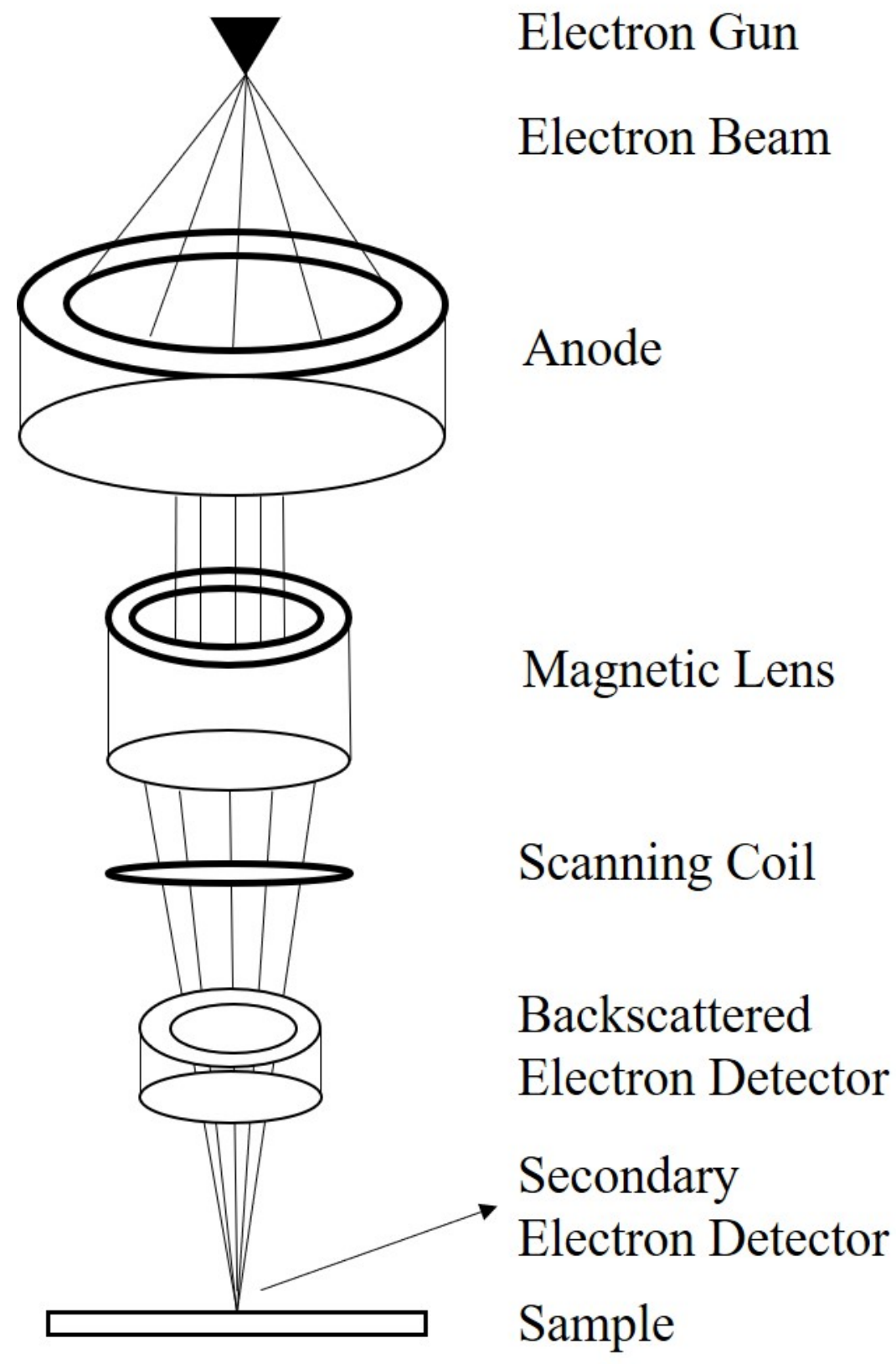

Figure 6: Schematic representation of principle of SEM. Figure adapted from Goldstein, J., et.al. Scanning Electron Microscopy and X-ray Microanalysis. Third Edition.

One of the important aspect that needs to be taken care of while taking images of biomolecules, examples proteins using SEM is burning or degradation of the sample due 
to the high energy electron bombardment. Also, when the nonconducting specimens are subjected to imaging, charge-up of specimen occurs and the secondary electrons are not emitted. As a result of which, image shift, deformation and abnormal contrast occurs. In order to prevent this, the specimens are first added into sputter and allowed to dry and then coated with conducting materials like gold or platinum. The average range for the coating varies from 1 to $20 \mathrm{~nm}$ which depends on the samples' stability.

In the following chapters 3 and 4, the morphology of the aggregates were confirmed using a cold field emission high resolution scanning electron microscope (Hitachi S-4700 FESEM). Samples were first diluted in MilliQ water and washed to remove the salts and buffer using Millipore Amicon® Ultra centrifugal filters ( $3 \mathrm{kDa}$ cut off). For this, samples were centrifuged at the speed of $7,000 \times \mathrm{g}$ at $4^{\circ} \mathrm{C}$ and repeated thrice. After the washing step, samples were aliquoted on stubs and left to dry at room temperature. Before imaging, samples were coated with platinum at the thickness of $10 \mathrm{~nm}$ using a sputter coater. Images were acquired using the acceleration voltage of $10 \mathrm{kV}$ and emission current of $5 \mu \mathrm{A}^{8}$.

\subsection{Cell based assays:}

Cell proliferation or viability is the most commonly employed technique to test the health of the cells. Cell metabolism and health can be affected by various physical and chemical agents. These agents can lead to cellular toxicity either by preventing protein synthesis, leading to cellular destruction, binding to receptors in irreversible manner, or inhibiting enzymatic reactions. This makes need of effective, cheap and reliable method to test cell viability as well as toxicity ${ }^{9}$. Different methods are available for testing cell viability or cytotoxicity like colorimetric, dye exclusion methods, fluorometric and luminometric 
assays. In the following sections two most commonly used assays for cell viability and cell toxicity as well as measurement of free radical will be discussed.

\subsubsection{MTS assay}

MTS assay is a colorimetric method that is used for determining the number of viable cells. This assay is commonly employed for testing cell proliferation, cytotoxicity and chemo sensitivity assays. The principle lies on formation of colored formazan from the conversion

of a tetrazolium salt (5-(3-carboxymethoxyphenyl)-2-(4,5-dimethyl-thiazoly)-3-(4sulfophenyl) tetrazolium, inner salt by the activity of living cells. The quantity of formazan product as measured by the amount of $490 \mathrm{~nm}$ absorbance is directly proportional to the number of living cells in culture. MTS assay has many advantages ranging from convenient, rapid, sensitive, economical and precise method for measuring in vitro cell proliferation assay. The disadvantages of this assay is the absorbance value measured at $490 \mathrm{~nm}$ is affected by the length of incubation, cell type, cell density and the ratio of reagent to cells in culture. Studies suggested that within the short time incubation i.e. upto 5 hours the linear relationship was observed for incubation time and absorbance ${ }^{9,10}$. 


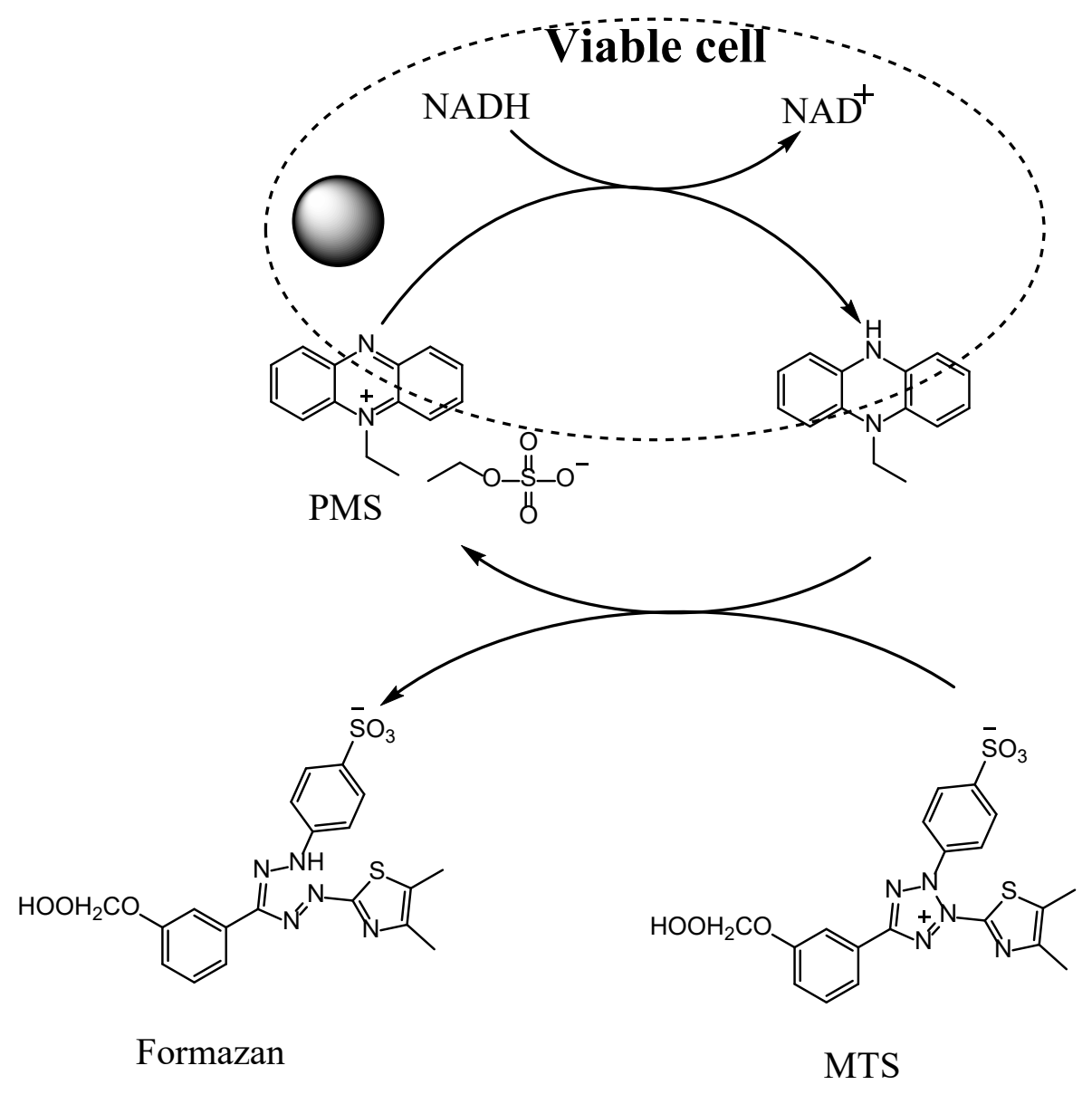

Figure 7: Mechanism of MTS assay. Structures were drawn using ChemDraw Pro 17.1 


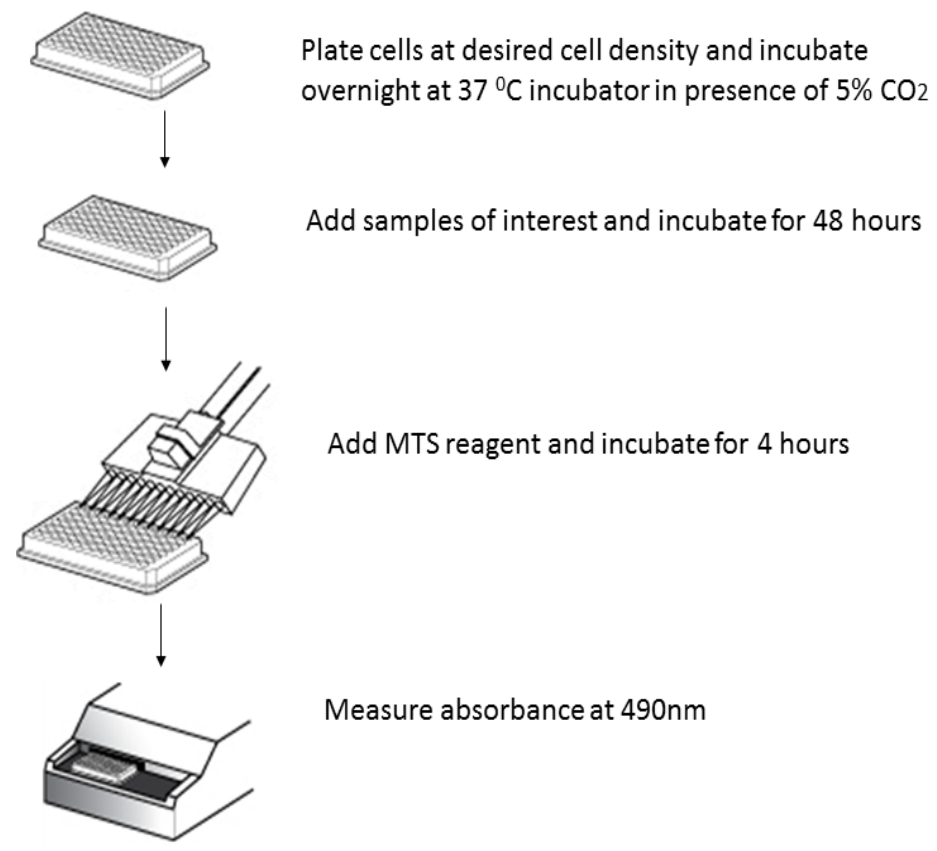

\section{Figure 8: Representation of general scheme for MTS assay.}

In the current thesis, MTS assay was employed to detect the cell viability in chapter 4 . The effect of aggregates amyloid beta protein was tested using SH-SY5Y human neuroblasto ma cells (from ATCC). In brief, SH-SY5Y cells was cultured in DMEM/F-12 medium containing $10 \%$ FBS and $100 \mathrm{U} / \mathrm{ml}$ penicillin-streptomycin. The cells were seeded at the density of $2 \times 10^{4}$ cells/well on 96 -well plates and incubated overnight at $37^{\circ} \mathrm{C}$ in $5 \% \mathrm{CO}_{2}$ humidified environment. All cellular experiments were conducted within first 10 passage of cells. Following day, the cells were washed twice with $1 \mathrm{X}$ PBS. Then, $100 \mu \mathrm{l}$ of fresh media containing samples at desired concentrations were prepared and added to cells in replicates of six. The cells containing samples were incubated at $37^{\circ} \mathrm{C}$ in $5 \% \mathrm{CO} 2$ humidified environment for $48 \mathrm{~h}$. Similarly, controls were prepared without adding any samples and containing only cells. After the incubation, MTS reagent ( CellTiter 96® AQueous One Solution Cell Proliferation (MTS) Assay kit (Promega)) was added to each 
well and incubated for $4 \mathrm{~h}$ at $37^{\circ} \mathrm{C}$. Finally, the absorbance was measured at $490 \mathrm{~mm}$ using a plate reader (BioTek Instruments, Inc.). Blanks were prepared for background subtraction and contained only media with test samples but without any cells.

\subsubsection{Lactate dehydrogenase (LDH) assay}

LDH is commonly employed technique for detecting the cytotoxicity in the cells. A cytosolic enzyme (LDH) is present in different types of cells and is released when there is damage to plasma membrane. The amount of released LDH can be quantified by using a coupled enzymatic reaction. In the first step, the conversion of lactate to pyruvate is catalyzed by the LDH through the reduction of NAD+ to NADH. Secondly, the tetrazolium salt (INT) is reduced by diaphorase using NAD+ leading to the formation of a red formazan product. The amount of LDH released in the media is directly proportional to the level of formazan formation which is measured by taking absorbance at 490nm. LDH assays has several features ranging from its reliability, quick and easy evaluation of cell death. On the other hand, when talking about the limitations, some of the compounds like serum (e.g. fetal calf serum) have inherent LDH activity leading to high background reading. So, serum free or low serum media is usually preferred for this assay $9,11,12$. 


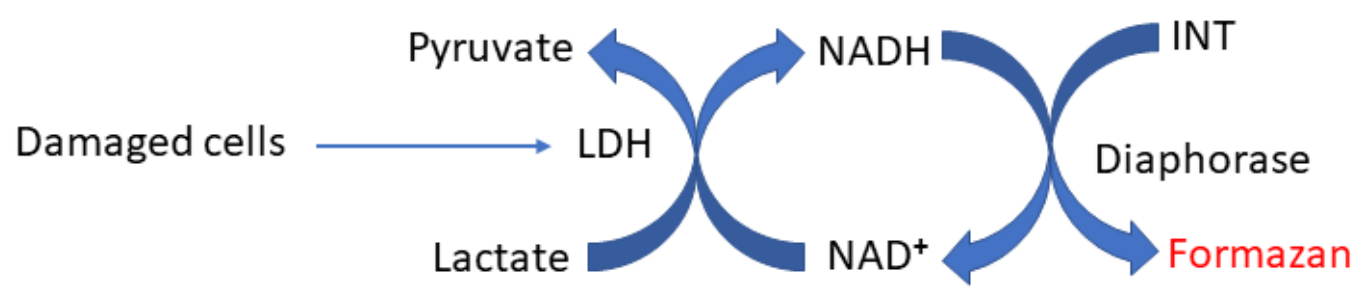

Figure 9: LDH assay principle.

LDH assay was employed to test the toxicity of aggregates to cells in chapter 4 . The effect of aggregates from amyloid beta peptides were tested using SH-SY5Y human neuroblastoma cells (from ATCC) and primary neuronal culture. In brief, SH-SY5Y cells was cultured in DMEM/F-12 medium containing 10\% FBS and $100 \mathrm{U} / \mathrm{ml}$ penicillinstreptomycin. The cells were seeded at the density of $2 \times 10^{4}$ cells/well on 96 -well plates and incubated overnight at $37^{\circ} \mathrm{C}$ in $5 \% \mathrm{CO}_{2}$ humidified environment. All cellular experiments were conducted within first 10 passage of cells. Following day, the cells were washed twice with $1 \mathrm{X}$ PBS. Then, $100 \mu \mathrm{l}$ of fresh media containing samples at desired concentrations were prepared and added to cells in replicates of six. The cells containing samples were incubated at $37^{\circ} \mathrm{C}$ in $5 \% \mathrm{CO}_{2}$ humidified environment for $48 \mathrm{~h}$. Similarly, controls were prepared without adding any samples and containing only cells. After the end of incubation, $10 \mu \mathrm{l}$ of lysis solution (CytoTox $96{ }^{\circledR}$ Non-Radioactive Cytotoxic ity Assay (LDH) Assay kit, Promega) was added to maximum LDH release control wells and incubated for 45 minutes at $37^{\circ} \mathrm{C}$ in $5 \% \mathrm{CO}_{2}$. Then, $50 \mu \mathrm{l}$ of supernatant from all the wells were transferred into a new plate and to each of those wells $50 \mu$ of $\mathrm{LDH}$ reagent was 
added. The plate was further incubated in dark for 30 minutes. After incubation, $50 \mu$ of stop solution was added to each well. Absorption at $490 \mathrm{~nm}$ were collected using a plate reader (BioTek Instruments, Inc.). Blanks were prepared for background subtraction and contained only media with test samples but without any cells.

\subsubsection{ROS measurement}

Reactive oxygen species (ROS) are formed by the reduction of oxygen molecules which contains unpaired electron (one or more) and are highly reactive in nature. The most commonly occurred ROS includes superoxide radical (O2-), hydroperoxyl radical (HO2-), hydroxyl radical (HO-), peroxyl radical (ROO-), alkoxyl radical (RO-), hydrogen peroxide (H2O2), singlet oxygen (1O2), nitric oxide (NO) and hypochlorous acid (HOCl). ROS plays an important role in health and diseases like they are known for their

protective and functional role in immune system. On the other hand, they are thought to play role in cancer, neurodegenerative diseases, atherosclerosis, and diabetes. Cells have antioxidant defense mechanism to overcome the production of free radicals in the cells. However, oxidative stress occurs when the balance of free radicals exceeds its defense mechanism. Reactive Oxygen Species (ROS) can be easily measured using 2', 7'dichlorofluorescin diacetate (DCFDA), where the nonfluorescent DCFDA is converted to the fluorescent compound in presence of ROS. The scheme for this reaction is shown in Figure 10. This assay is very sensitive to changes in redox state of the cell and is easy to perform and is an inexpensive method which can be used to detect the changes in ROS over the time $13,14,15$. 


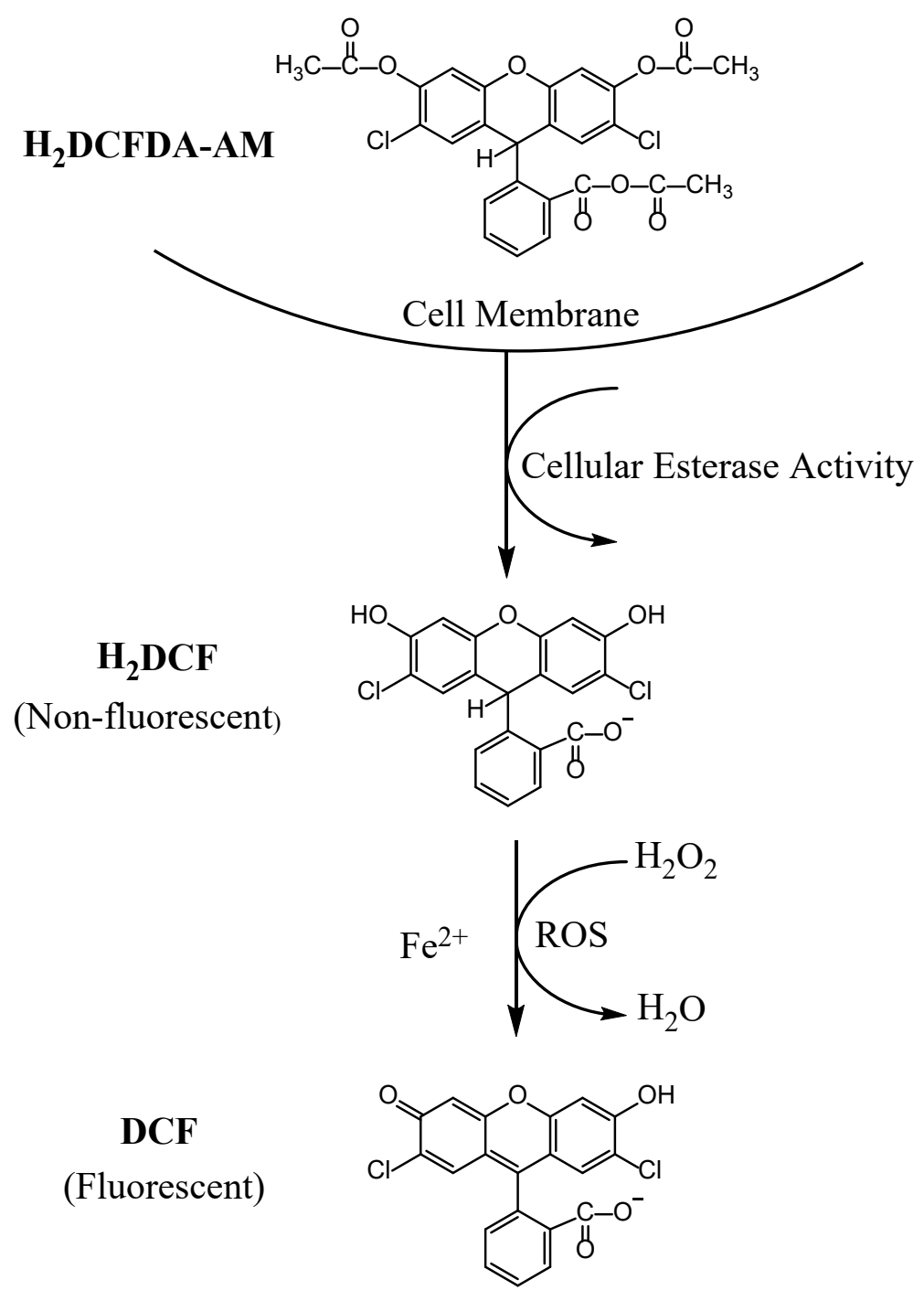

Figure 10: Reaction showing the conversion of DCFDA to fluorescent compound by ROS. Structures were drawn using ChemDraw Pro 17.1

In chapter 4, we used 2',7'-dichlorofluorescin diacetate (DCFH-DA) fluorescence assay to measure the ROS in the cell. Like MTS and LDH assay, SH-SY5Y human neuroblasto ma cells (from ATCC) were cultured and were plated at $2 \times 10^{4}$ cells $/$ well on 96 -well plates and allowed to grow overnight. The next day, cells were washed with $1 \mathrm{X}$ PBS buffer $(\mathrm{pH}$ 7.4) twice. Then, cells were incubated with $20 \mu \mathrm{M}$ DCFH-DA for 45 minutes at $37^{\circ} \mathrm{C}$ in 
dark. After incubation, the cells were washed twice with 1X PBS (pH 7.4) and then $100 \mu l$ of fresh media containing peptide samples were added to DCFH-DA (Sigma) treated cells in six replicates and incubated for $24 \mathrm{~h}$ at $37{ }^{\circ} \mathrm{C}$ in $5 \% \mathrm{CO} 2$ humidified environment. Cells without any treatment and blanks were also incubated under identical condition. For positive control, cells after DCFH-DA treatment were incubated with $50 \mu \mathrm{M}$ of t-butylhydroperoxide (tBHP) for $4 \mathrm{~h}$ at $37^{\circ} \mathrm{C}$. Finally, the fluorescence intensity was measured using fluorescence plater reader (Fluoroskan Ascent, Thermo Scientific) at excitation wavelength of $485 \mathrm{~nm}$ and emission wavelength of $538 \mathrm{~nm}$.

\subsection{UV-Vis spectroscopy}

UV- Vis spectroscopy deals with the measurement of absorbance in the UV and visible range ranging from $200-800 \mathrm{~nm}$. While fluorescence spectroscopy measures the transitions from excited to ground state, absorption spectroscopy measures the transition from ground to excited state. The principle of UV-absorbance is based on the Lambert Beer's Law. According to the Beer-Lambert Law, the absorbance of a solution is directly proportional to the concentration of the substance present in that solution and is expressed as,

$$
A=\varepsilon_{c} 1
$$

Where, $\mathrm{A}=$ absorbance; $1=$ optical path length expressed in $\mathrm{cm} ; \varepsilon=$ molar extinction coefficient, expressed in $\mathrm{M}-1 \mathrm{~cm}-1 ; \mathrm{c}=$ molar concentration. The three aromatic amino acids namely Phenylalanine, Tyrosine and Tryptophan are responsible for the absorption of light in UV range. Protein shows the absorption maxima around the wavelength of $280 \mathrm{~nm}$ due to the presence of these three aromatic amino acids. Among them phenylalanine 
contributes the least due to its lower absorptivity and is usually not accounted for protein UV absorbance. UV-Vis spectroscopy is also used to measure the concentration of a sample. It is widely used for the quantitative analysis and also for measurements of conformational changes of proteins and nucleic acids, ligand-binding reactions. Since, the absorbance not only depend on the structure but also only the local environment, this method is also used to quantify the protein aggregation. This is basically accomplished by measuring the turbidity of the solution. The increase in turbidity leads to increase in absorbance which signifies the increase in protein aggregates.

\subsection{Fluorescence spectroscopy}

Fluorescence is a slow process which usually occurs in the order of $10^{-9}$ to $10^{-7}$ seconds. When an molecule receives an energy of certain wavelength it is excited from ground state to its excited state. Since, the excited electron is not stable in its excited state, it returns back to the ground state emitting a light of certain energy which is measured as a fluorescence. Fluorophores are the fluorescent compounds and are aromatic in nature. Jablonski diagram best describes the phenomenon of absorption, fluorescence and phosphorescence which is shown in Figure 11. As shown in the figure below, the radiative transitions are represented by the solid arrows and the non-radiative transitions are represented by the dashed arrows. The notation S0, S1 and S2 represents the different energy levels namely ground, first and second respectively. Internal conversion and Intersystem crossing are an example of the non-radiative transitions. Intersystem crossing occurs between the states of different spin multiplicity. Coupling of vibrational state of higher electronic state to lower electronic states results into internal conversion. For 
Emission of photons leads to release of energy and is observed when electron return to different vibrational level of S0 from S1. This emission is measured as fluorescence. Phosphorescence is generally observed when the electron from triplet state return back to ground state. Compared to S1, triplet state is lower energy level hence phosphorescence has longer emission wavelengths than fluorescence.

Intrinsic and extrinsic fluorescence are the most commonly used fluorescence methods to detect the changes in protein structure. Fluorescence spectroscopy is widely employed technique in studying the protein folding due to its sensitive detection of emission spectra, which is greatly affected by the change in local environment as well as the structure of the fluorophore.

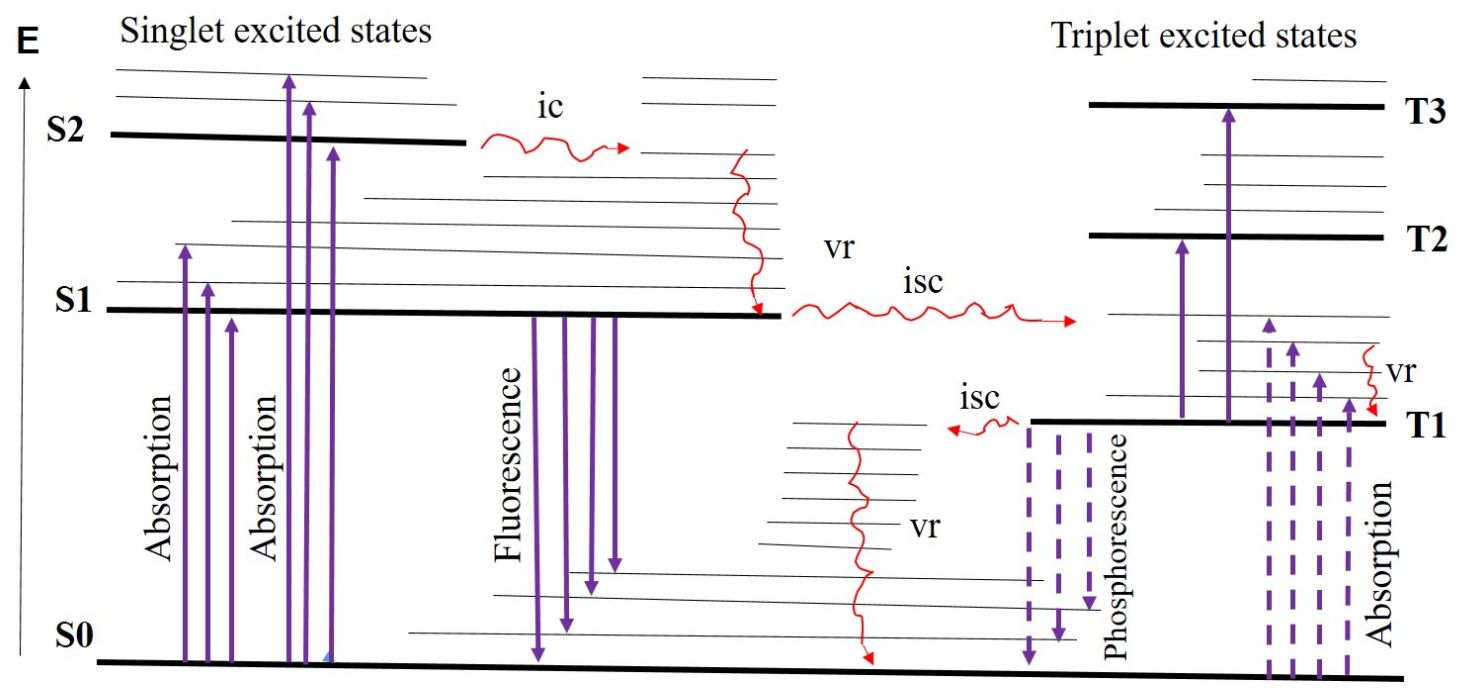

Figure 11. Jablonski diagram representing the absorption, fluorescence and phosphorescence. The different electronic states are represented by S0 (ground), S1 (first), S2 (second), and T1 (triplet) state. Fluorescence and Phosphorescence represents the radiative transitions and internal conversion, intersystem crossing and vibrational 
relaxation represents the non-radiative transitions. Figure Adapted from Alvaraz.V. et.al. $2012^{21}$.

Intrinsic fluorescence of a protein is the mainly contributed by two aromatic amino acids tyrosine and tryptophan. Due to the highest absorption coefficient of tryptophan compared to other two aromatic amino acids Phenylalanine and Tyrosine (Figure 2.9), intrinsic fluorescence is measure of tryptophan residue. The fluorescence contributed by the tryptophan is highly sensitive to local environment as a result of which, it can be blue shifted which is towards shorter wavelength when the protein is under the native condition and red shifted fluorescence is observed when the protein is unfolded. This characteristic can be used to monitor the conformation change of a protein and measure the protein unfolding phenomenon. During folding of the protein, the hydrophobic residues are buried down in the protein core and is not exposed to the solvent. . The intrinsic fluorescence can be used to study the protein denaturation in presence of denaturants like Guanidine $\mathrm{HCl}$ (GdnHCl) at different $\mathrm{pH}$. Figure 13, Shows the chemical denaturation of cytochrome $\mathrm{C}$ protein at $\mathrm{pH} 4,7$ and 10 . The protein cytochrome $\mathrm{C}$ in presence of denaturant guanid ine $\mathrm{HCl}(\mathrm{GdnHCl})$, shows the increase in the fluorescence intensity with the increase in the concentration of the guanidine $\mathrm{HCl}$. This is due to exposure of the hydrophobic residue especially Tryptophan to the aqueous environment during the process of protein unfold ing. The peak fluorescence intensity thus observed can be used to plot the denaturation curve which is in the sigmoidal shape (Figure 13). The native fit line, transition fit line and denatured fit line obtained from the curve helps to calculate the different thermodynamic parameters like $\Delta \mathrm{G}, \Delta \mathrm{H}, \Delta \mathrm{S}, \mathrm{Cm}$. 
<smiles>NC(Cc1ccccc1)C(=O)O</smiles>

Phenylalanine<smiles>NC(Cc1ccc(O)cc1)C(=O)O</smiles>

Tyrosine<smiles>NC(Cc1c[nH]c2ccccc12)C(=O)O</smiles>

Tryptophan

Figure 12: Structure of three aromatic amino acids Phenylalanine (Phe), Tyrosine (Tyr) and Tryptophan (Trp). Structures were drawn using ChemDraw Pro 17.1
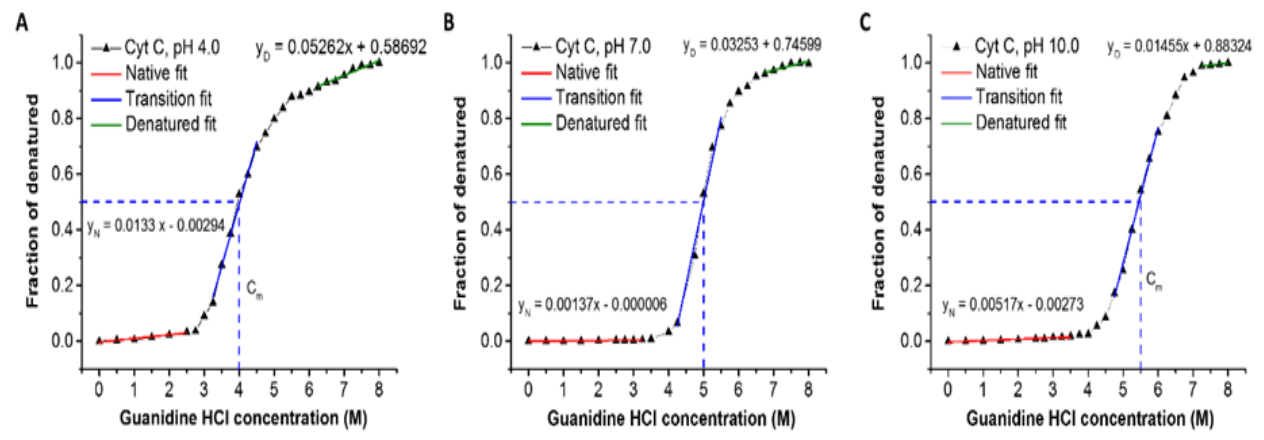

Figure 13. Chemical denaturation curves of Cytochrome $\mathrm{C}$ at $\mathrm{pH} 4.0,7.0$, and 10.0 as a function of guanid ine $\mathrm{HCl}$ concentration. Fluorescence emission of proteins were collected at $300-600 \mathrm{~nm}$, with excitation at $280 \mathrm{~nm}$. The peak intensities at $355 \mathrm{~nm}$ were normalized as fraction of denatured. Concentration of protein was $0.3 \mathrm{mg} / \mathrm{ml}$ at all $\mathrm{pH}$.

Extrinsic fluorophores are the compounds that are covalently linked to ligands or proteins and associated with the transfer of fluorescence energy. Electrostatic and hydrophobic interactions are the major non-covalent interactions that are involved during the interaction of extrinsic fluorophores with proteins. These dyes are very sensitive to the local environment. Extrinsic fluorophores help in characterization of protein such as it can be used to study the changes in conformation, structure, size, kinetics of a protein, surface 
hydrophobicity, probe active sites of enzyme, detect intermediates of molten globule, monitor folding and unfolding of protein, and its species that are produced during the process of aggregation 22 .

Table 2.1. Common extrinsic fluorescent dyes and their applications /

\begin{tabular}{|l|l|}
\hline Dyes & Applications \\
\hline Thioflavin T (ThT) & Detect the morphology of aggregates \\
\hline ANS & $\begin{array}{l}\text { Protein folding, Aggregation Kinetics, Surface } \\
\text { hydrophobicity }\end{array}$ \\
\hline Bis-ANS & $\begin{array}{l}\text { Protein conformation , Surface hydrophobicity, Protein } \\
\text { folding and unfolding kinetics }\end{array}$ \\
\hline Congo Red & $\begin{array}{l}\text { Amyloid fibril analysis } \\
\text { hydrophobicity unfolding/folding }\end{array}$ \\
\hline Nile Red & changes in protein conformation, surface \\
\hline
\end{tabular}




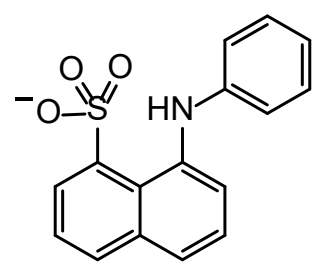

ANS

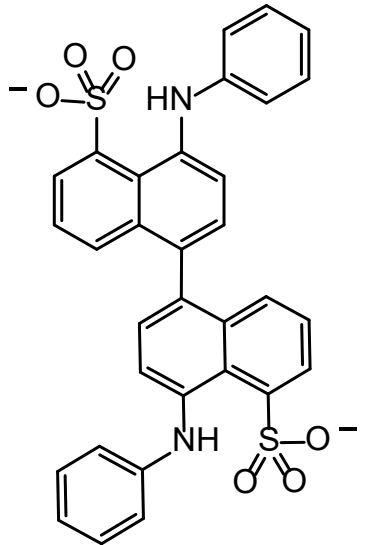

Bis-ANS

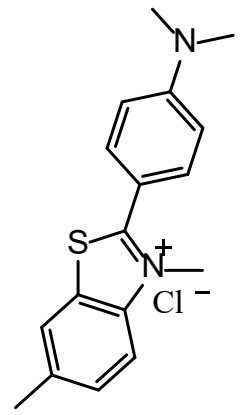

ThT

Figure 14: Some common extrins ic fluorescent dyes used in protein aggregation studies. Structures were drawn using ChemDraw Pro 17.1

ANS (1-anilinonaphthalene-8-sulfonate) and Bis-ANS (dimeric analogon 4, 4'-bis-1 anilinonaphthalene-8-Sulfonate), are the fluorescent dyes that are used for the protein characterization. The dye ANS is the most commonly used dye to measure the surface hydrophobicity. ANS fluorescence is highly sensitive to the local environment and shows increase in fluorescence in non-polar environment whereas in polar environment it is weakly fluorescent. Also, the fluorescence depends on the polarity, viscosity and temperature of dye's environment. The increase in quantum yield with the blue shift of emission maxima results from the decrease in dielectric constant of the solvent. The mechanism for binding of ANS to protein is through the hydrophobic and electrostatic interactions. However, the sulfonate group of ANS pairs with the positive amino acids on the proteins like lysine, arginine and histidine through ion-pairing making it the 
predominant interactions. Apart from this the weak forces like van der Waals interactions is also necessary to make the ions pairs stable. On the other hand, the major interaction between Bis-ANS and protein is the hydrophobic interaction. The binding affinity may vary due to the different sizes of the dye and the number of binding sites present on the protein. Both dyes have similar intrinsic fluorescence properties but when compared to the UV absorption and fluorescence emission, Bis-ANS displays UV-absorption maximum as well as emission shifted towards longer wavelength. Though the interaction in Bis-ANS is driven by the hydrophobic interaction it does not bind strongly with the organized structures. For its strong binding, it requires the molten globule like structures and has weak reactivity towards amyloid fibrils. Therefore, ANS and Bis-ANS provides differe nt information in protein aggregation. Bis-ANS provides information on flexibility of the structure of aggregates i.e. whether it forms loose or tight structures. Tht (Thioflavin $\mathrm{T}$ ) is a fluorescent dye that is most commonly used to characterize the amyloid fibrils. The fluorescent intensity of Tht dye is less depended on the polarity and affected by the viscosity and rigidity of the environment. The interaction of the Tht to amyloid fibril is driven by the hydrophobic interactions with the side chains of amino acids. Now it is evident that Tht not only binds to the fibrils but also to the non-fibrils structure resulting into the increase in fluorescence.

In chapter 4, Horiba Jobin Yuon spectrofluorometer (Fluoromax-4) was used for fluorescence measurement. All dyes stocks were prepared in $100 \%$ ethanol and were diluted in respective buffers to prepare working stocks for incubation with samples. UVVis spectrophotometer was used to measure the concentration of each dyes. The extinction coefficient used was ANS $\varepsilon 350 \mathrm{~nm}=5,000 \mathrm{M}-1 \mathrm{~cm}-1$, bis-ANS $\varepsilon 385 \mathrm{~nm}=16,790 \mathrm{M}-1 \mathrm{~cm}-$ 
1. Both dyes were used at the final concentration of $10 \mu \mathrm{M}$ and incubated with $10 \mu \mathrm{M}$ of protein samples on ice for 15 minutes before acquiring emission spectra. The emission spectra were collected from $400-700 \mathrm{~nm}$ range with excitation at $360 \mathrm{~nm}$ for bis-ANS and $380 \mathrm{~nm}$ for ANS dye. Samples were run in triplicates with incubation in dark with dyes.

\subsection{Immunofluorescence}

Immunofluorescence is the most commonly used technique to detect the localization and endogenous expression level of protein of interest by using the specific antibodies that is conjugated with the fluorescent dye. Immunofluorescence is a form of immunohistochemistry which uses fluorophores to detect the target. This technique can be used on cultured cells and individual cells, tissue sections. Several factor accounts into its application ranging from type of cells, nature of antigen, specificity and sensitivity of antibodies, permeabilization and fixation method, imaging of the cell. The fluorescence detection method involves the use of direct or indirect method ${ }^{23}$. The direct method uses of single antibody that is linked to the fluorophore. The antibody recognizes the target antigen/ molecule and binds specifically to it which can be detected using the fluorescence microscope. This method has advantage over the indirect method because of the direct conjugation with the fluorophore which minimizes the number of staining step as well as problem of background signal, antibody cross-reactivity or non-reactivity. However, direct method is less sensitive compared to the indirect method. The indirect method on the other hand uses two antibodies primary and secondary. The primary antibody binds to the target molecule and secondary antibody contains the fluorophore which recognizes the primary antibody and binds to it. This method requires more steps than direct method. 
In Chapter 3, we applied immunofluorescence technique to detect the cellular localization of our TDP-43 protein using $\mathrm{N}$ and C-terminal antibodies. Also, in chapter 4 , to detect the effect of Abeta peptides on primary neuronal culture we employed immunofluorescence method in addition to cellular toxicity. The specific details on procedure is described under respective chapters.

\subsection{References:}

1. Bornhorst, J.A.; Falke, J.J., Purification of Proteins Using Polyhistidine Affinity Tags. Methods Enzymology 2000, 326, 245-54

2. Crowe, J.; Masone, B.S.; Ribbe, J., One-step purification of recombinant proteins with the 6xHis tag and Ni-NTA resin. Methods in Molecular Biology 1996, 58, 491-510.

3. Chrambach, A.; Rodbard, D. Polyacrylamide Gel Electrophoresis. Science 1971, 172 (3982), 440-451

4. Mahmood, T.; Yang P.C., Western Blot: Technique, Theory, and Trouble Shooting. North American journal of medical sciences 2012, 4(9), 429-34.

5. Goldstein, J.; Newbury, D.; Joy, D. C.; Charles E. Lyman, Patrick Echlin, Eric Lifshin, Linda Sawyer, J.R. Michael, Scanning Electron Microscopy and X-Ray Microanalys is. Third Edition ed.; Kluwer Academic/Plenum Publishers: 2009.

6. Egerton, R.F., Physical principles of electron microscopy: an introduction to TEM, SEM and AEM. Springer: 2005, 1-202.

7. Dumont, M.; Borbély, A.; Kostka, A.; Sander, P.M.; Pyzalla, K., Characterization of Sauropod Bone Structure. 2009, 150-170 
8. Yang, M.; Dutta, C.; Tiwari, A., Disulfide-Bond Scrambling Promotes Amorphous Aggregates in Lysozyme and Bovine Serum Albumin. Journal of Physical Chemistry B 2015, 119, 3969-81.

9. Aslantürk, O.S., In Vitro Cytotoxicity and Cell Viability Assays: Principles, Advantages, and Disadvantages. Genotoxicity. 1-17

10. Berg, K.; Zhai, L.; Chen, M.; Kharazmi, A.; Owen, T.C., The use of a water soluble formazan complex to quantitate the cell number and mitochondrial function of Leishmania major promastigotes. Parasitology Research. 1994, 80:235-239

11. Decker, T.; Lohmann-Matthes, M.L., A quick and simple method for the quantitation of lactate dehydrogenase release in measurements of cellular cytotoxicity and tumor necrosis factor (TNF) activity. Journal of Immunological Methods. 1988, 160:81-88

12. Lappalanien, K.; Jaaskelainen, L.; Syrjanen, K.; Urtti, A.; Syrjanen, S., Comparison of cell proliferation and toxicity assays using two catronic liposomes. Pharmaceutical Research. 1994, 11:1127-1131

13. Eruslanov, E.; Kusmartsev, S., Identification of ROS using oxidized DCFDA and flowcytometry. Methods Molecular Biology. 2010, 594:57-72

14. Gomes, A.; Fernandes, E.; Lima, J.L., Fluorescence probes used for detection of reactive oxygen species. Journal of Biochemical and Biophysical Methods. 2005 65(23):45-80.

15. Murrant C.L.; Reid M.B., Detection of reactive oxygen and reactive nitrogen species in skeletal muscle. Microscopy Research and Technique. 2001, 55:236 -248 
16. Antonie J.W.G. Visser.; Rolinski O.J., Basic Photophysics. Laboratory of Biochemistry, Microspectroscopy Centre, Department of Physics, University of Strathclyde.

17. Arakawa, T.; Philo, J.S.; Ejima, D.; Tsumoto, K.; Arisaka, F., Aggregation analysis of therapeutic proteins, part 1: General aspects and techniques for assessment. Bioprocess International 2006, 4: 42-49

18. Nelson, D.L.; Cox, M. Lehninger Principles of Biochemistry. Fifth Edition ed.; W.H. Freeman and Company: New York, 2008.

19. Schmid, F.-X., Biological Macromolecules: UV-visible Spectrophotometry. In $e L S$, John Wiley \& Sons, Ltd: 2001.

20. Lakowicz, J.; Principles of Fluorescence Spectroscopy. Third Edition ed.; Springer 2010

21. V. Alvarez, et.al. SiPMs coated with TPB: coating protocol and characterization for NEXT. JINST 2012, 7, P02010.

22. Hawe, A.; Sutter, M.; Jiskoot, W., Extrinsic fluorescent dyes as tools for protein characterization. Pharmaceutical Research 2008, 25, 1487-99.

23. Donaldson, J.G., Immunofluorescence Staining. Current Protocol in Cell Biology. 2015 1; 69:4.3.1-7. 


\section{Chapter 3}

\section{Characterizing the physicochemical properties of TDP-43 protein to}

discern its role in neurodegenerative diseases

Rashmi Adhikari ${ }^{\mathrm{a}}$, Jagadeesh Janjanama ${ }^{\mathrm{a}}$, Colina Dutta ${ }^{\mathrm{a}}$, and Ashutosh Tiwari ${ }^{\mathrm{a}, *}$

a Department of Chemistry, Michigan Technological University, Houghton, Michigan 49931, United States

$\uparrow$ This chapter contains materials that is under preparation for submission in a peer-review journal. 


\subsection{Introduction:}

Amyotrophic lateral Sclerosis (ALS) is an adult-onset neurodegenerative disease characterized by the premature loss of upper and lower motor neurons leading to death of motor neuron in the brain, brainstem and spinal cord. Patients with ALS have life expectancy of only 3-5 years after the onset of disease ${ }^{1}$. About $10 \%$ of the ALS cases are familial and rest remains to be sporadic ${ }^{2-4}$. Frontotemporal Lobar degeneration (FTLD) is the second common form of cortical dementia observed in patients below the age of $65^{5,6}$. FTLD leads to loss of neurons in the frontal and temporal cortex and is characterized by the alteration in personality, social behavior, and language dysfunction. The hallmark of ALS and FTLD includes ubiquitin positive abnormal protein aggregates in the brain of affected patients ${ }^{7,8}$. The presence of ubiquitinated aggregates of transactive response DNA binding protein $43 \mathrm{kDa}$ (TDP-43) in FTLD and ALS patients in 2006 raised a lot of interest in understanding the role of this protein in the pathogenesis of ALS and FTLD diseases 9,10,11. The pathogenic mechanism involved in ALS has not been solved yet but in case of FTLD and ALS patients, TDP-43 is known to be accumulated in the cytoplasmic inclusion of affected areas of the spinal cord and brain ${ }^{12}$. The common pathological features with ALS and FTLD include the presence of cytoplasmic inclusions with ubiquitin and TDP-43 positive, $\alpha$-synuclein and tau negative. The primary cause for the ALS being identified as the dominant mutation in TDP-43 resulted in further research being directed toward understanding the role of TDP-43 in neurodegeneration ${ }^{13,14}$. In addition to ALS and FTLD, TDP-43 is also involved in several other diseases like Alzheimer's, Parkinson's, Lewy Body and Huntington ${ }^{15-18}$. 
A 414- amino acid residue protein, TDP-43 is structurally distinguished into N-terminal, C-terminal and two-RNA binding domains (RRM1 and RRM2) ${ }^{19-27}$. Being a protein of nuclear origin, TDP-43 protein plays a significant role in health and diseases. Under normal conditions TDP-43 performs a wide variety of functions basically in the regulation of mRNA pathways which I will discuss in following paragraphs.

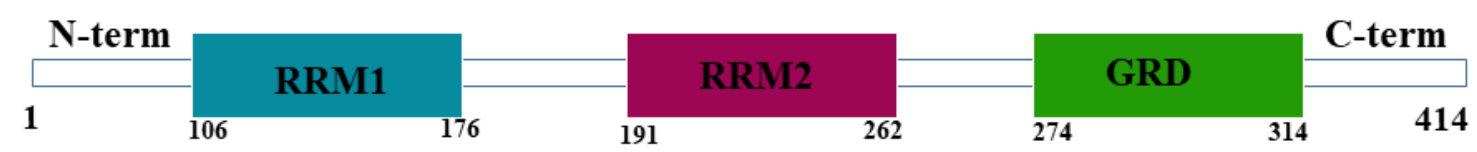

Figure 1: Schematic representation of TDP-43 showing different regions.

\section{Biological Functions of TDP-43}

TDP-43 is known for its role in protein-protein interaction which occurs through the glycine rich C-terminal region. TDP-43 is associated with the members of hnRNP family like hnRNP A2/B1, hnRNP A1, hnRNP $\mathrm{C} 1 / \mathrm{C} 2$ and hnRNP $\mathrm{A} 3$, interaction of which are dependent on C-terminal region. TDP-43 plays a wide variety of biological functions mainly in the regulation of RNA pathways as summarized in the figure $3^{17,19}$.

\section{a. TDP-43 as a transcriptional regulator}

The conversion of DNA to RNA is regulated by the transcriptional regulator. TDP-43 is well conserved among invertebrates and mammals and is expressed in almost all tissues. First identified in 1995, as a transcriptional inactivator of Human immunodeficiency virus type 1 (HIV-1), TDP-43 role is known in transcription repression. Binding of TDP-43 with the double stranded HIV TAR DNA repeats takes place through its RRMs domain. 
Presence of polypyrimidine rich region in TAR DNA facilitates its binding with TDP-43 as a result of which recruitment of TATA binding protein to TATA element is inhibited. TDP-43 also binds with mouse SP-10 gene promoter and is involved in transcription repression in spermatids ${ }^{18,19,28-30}$.

\section{TDP-43 in splicing regulation}

Splicing is the process by which the introns are removed and the exons are joined together to produce the mature messenger RNA. TDP-43 is known to be involved in splicing regulation of several gene transcripts such as Cystic fibrosis transmembrane conductance regulator (CFTR), TARDBP, FUS, SNCA ( $\alpha$-synuclein), HTT (Huntingtin), and APP (Amyloid precursor protein). The splicing pattern of the targets is known to be altered by the level of TDP-43, i.e. overexpression or its depletion. The glycine rich C-terminal domain of TDP-43 is involved in the interaction with different proteins involved in splicing $11,6,7$. Being a member of hnRNP family, TDP-43 is found to interact with various members of hnRNPs like hnRNP A2/B1, hnRNP A1, hnRNP C1/C2 and hnRNP A3. The C-terminal region of TDP-43 is involved in binding with hnRNPs and the hnRNPs regulate the splicing. TDP-43 also regulates survival of motor neuron, apolipoprotein A2 and serine/arginine-rich splicing factor $2^{11,16,18}$.

\section{b. TDP-43 in micro-RNA processing}

The presence of TDP-43 in Drosha complex (a nuclear RNase type-III protein) together with FUS/TLS as well as its association with the perichromatin fibers first raised the possibility of TDP-43 role in miRNA biogenesis. Further studies suggested the 
dysregulation of several miRNAs upon depletion of TDP-43. An example of this includes let-7b, miR-9, miR-132, miR-520, miR-663 etc. ${ }^{11,19,20}$. TDP-43 is involved in dysregulation either by binding with the miRNAs or its precursors molecules. However, TDP-43 is involved in controlling the stability of Drosha protein during the neuronal differentiation.

\section{c. Role of cytosolic TDP-43 in regulation of RNA subcellular localization}

TDP-43 is a nuclear protein and can shuttle between nucleus and cytoplasm. It performs a diverse role in nucleus and cytoplasm. In the cytosol, TDP-43 regulates the subcellular localization of RNA as well as its transport, translation and stress-granule formation. TDP43 role in translation repression is also known by interacting with proteins involved in translation.

During the condition of stress, the formation of cytoplasmic foci represents TDP-43 as a component of stress granules. Under the condition of stress TDP-43 translocate into cytoplasm and colocalizes with stress granules and may play role in mRNA stability as well as its trafficking. However, how the TDP-43 positive stress granules play role is yet to be identified ${ }^{16,} 29,30,33$.

\section{d. Role of TDP-43 in mRNA stability}

Though identified to be highly localized in the nucleus and perform its functional activity, role of TDP-43 is also identified in the cytoplasm. TDP-43 is involved in the transport, stability and translation of mRNA in the cytoplasm. The role of TDP-43 in mRNA stability was first identified in two targets namely human low molecular weight neurofilament and 
the histone deacetylase HDAC6 transcripts. More mRNAs are now known whose stability is altered by TDP-43. An example of this includes: Add2, VEGFA and GRN, and IL- $6{ }^{34}$. The maintenance of neuronal activity and synaptic plasticity in the neurons requires the transport of mRNA into axons and dendrites. In a study reported by Fallini et.al. 2012, TDP-43 was known to be actively transported along axons in primary motor neurons. Also, TDP-43 colocalizes with other RNA binding proteins like SMN and FMRP, in RNA granules 34 .

\section{e. Role of TDP-43 in Genome stability}

TDP-43 is important for early mouse embryogenesis, the homozygous disruption of tar DNA binding gene are embryonically lethal. The expression of TDP-43 is tightly regulated. It has been observed that the knockdown of TDP-43 leads to death of differentiated neurons as well as tumor cells. Similarly, the lethality of the second-larvae stage in Drosophila occurs due to TDP-43 disruption while semi-lethality was reported by some other groups 35 . 


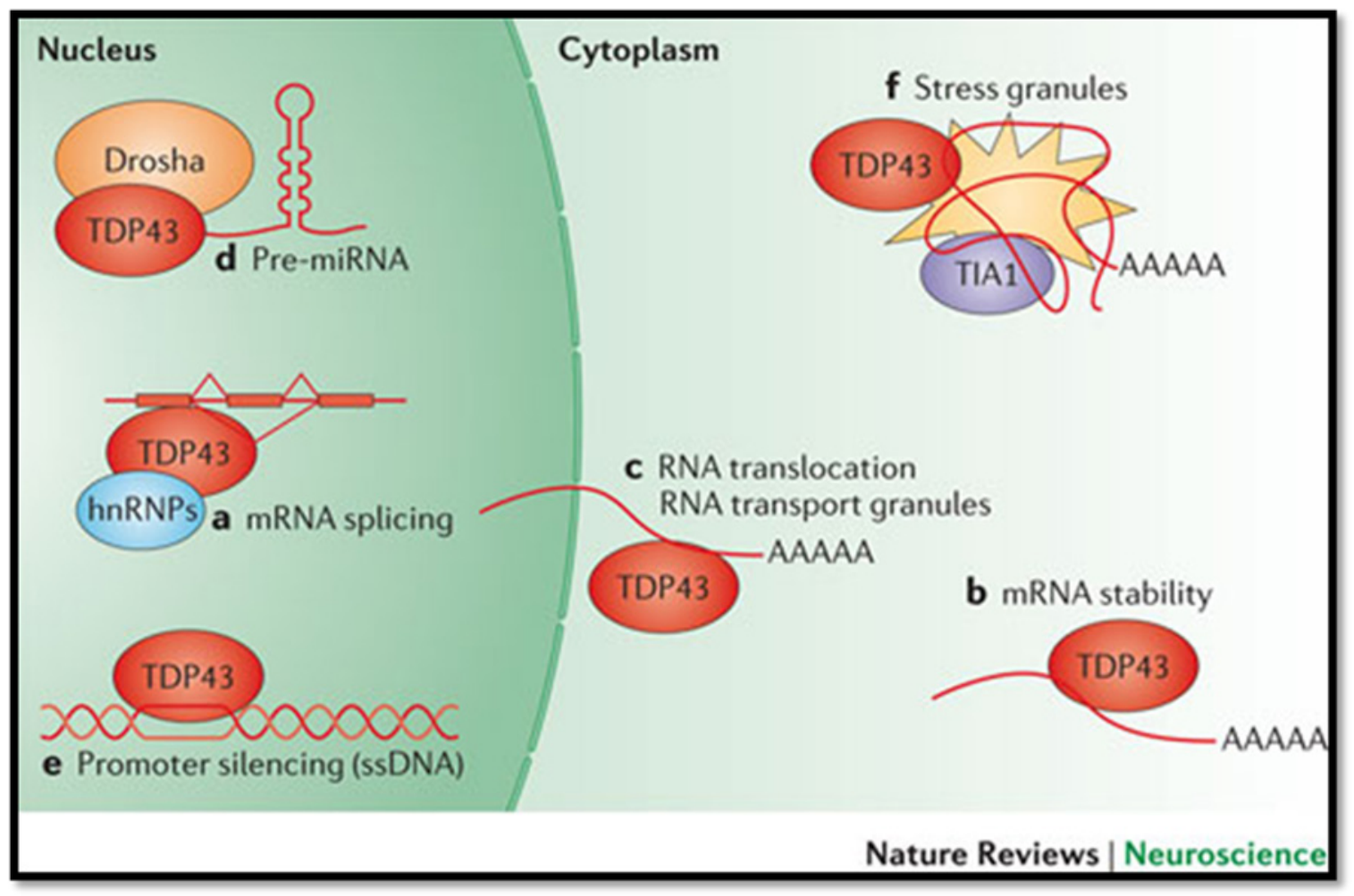

Fig 2: Biological functions of TDP-43. TDP-43 plays a wide variety of biological functions mainly in the regulation of RNA pathways ${ }^{28}$. (a) TDP-43 being a component of heterogenous ribonucleoprotein (hnRNPs) participates in the pre-mRNA splicing regulation. (b)TDP-43 binds to the $3^{\prime}$ untranslated region and affects the mRNA stability as well as turnover (c) TDP-43 localize with the RNA granules and is involved in mRNA trafficking as it can shuttle between nucleus and cytoplasm. (d) TDP-43 is part of the Drosha complex and is involved in processing of miRNAs (e) TDP-43 can act as a transcriptional repressor by binding to the promoter sequences of single stranded DNA. (f) TDP-43 stabilizes the mRNA under the condition of stress by colocalizing with the stress granules. (Figure reprinted from Lee, et.al. Used with permission from the publisher) 
TDP-43 distinguished into different domains, possess specific roles. Compared to other domains, N-terminal domain function was explored late and was found to be crucial for regulating its self-interaction. Also, N-terminal region is reported to regulate TDP-43 aggregation. RRM1 and RRM2 domain is involved in binding to RNA/DNA sequence and protein-protein interaction takes place through Glycine rich C-terminal domain ${ }^{11,18,19,32}$. TDP-43 plays an important role in health and disease. Being involved in the pathways of RNA regulation, TDP-43 under pathological conditions is involved in several neurodegenerative diseases.

\section{TDP-43 in neurodegeneration}

TDP-43 first identified to be involved in neurodegenerative disease as a ubiquitinated neuronal cytoplasmic inclusions in FTD and ALS patients is also known to be involved in several neurodegenerative diseases like Alzheimer's, Huntington, Parkinson's Lewy bodies ${ }^{37-42}$. In $97 \%$ cases of ALS, TDP-43 inclusions are known to be positive. The common pathological features include ubiquitinated and hyperphosphorylated aggregates in the cytoplasm, devoid of TDP-43 in the nucleus. All of the neurodegenerative diseases that are associated with deposition of TDP-43 are commonly termed as 'TDP-43 proteinopathies' which possesses common pathological features. Intracellular and extracellular accumulation of protein aggregates comprises the major characteristics of wide variety of neurodegenerative diseases. Although neurodegenerative diseases like Alzheimer's disease (AD), Parkinson's disease (PD), Huntington's disease (HD), Amyotrophic lateral sclerosis (ALS) have distinct proteins associated with them such as SOD1 with ALS, Amyloid $\beta$ with Alzheimer's etc. There's a renewed focus on TDP-43 as 
its role and importance in all these diseases have been reported. There lies a common pathogenic mechanism involved in many neurodegenerative diseases, which involve the central nervous system amyloidosis because of aggregation and deposition of misfolded protein. During this process, the soluble proteins are transformed into the insoluble and filamentous polymers, which are rich in cross- $\beta$-pleated sheet structures. These structures accumulate in the nuclei or cytosol as a fibrillar amyloid deposits in the affected brain cells or in extracellular space. For the majority of neurodegenerative diseases, the effective method of treatment is lacking due to difficulty in identifying the disease-causing toxic fold of the protein. As TDP-43 is involved in multiple neurodegenerative diseases it has been target of study by basic and clinical researchers, as well as industry to understand its role in the disease process.

The cytoplasmic localization of TDP-43 is the common feature observed in neurodegenerative diseases. Apart from this, the modifications like hyperphosphorylation, ubiquitination and $\mathrm{C}$-terminal truncation is observed in several neurodegenerative diseases. Most of the mutations in TDP-43 is present in C-terminal domain. The hyperphosphorylated and ubiquitinated C-terminal fragment of TDP-43 comprises the major component in the neuronal inclusion bodies in case of ALS and FTLD patients. Experiments carried out in cultured cells showed that the prion -like activity is present in TDP-43 and is capable of converting the normal cells into the abnormal form ${ }^{32}$. Due to the importance of C-terminal domain, there is increasing demand to examine the sequence of this domain. Analysis by PONDR PROGRAM, which predicts the naturally disordered region, identified that about $80 \%$ of the $\mathrm{C}$-terminal region is structurally disordered ${ }^{41}$. The intrinsically disordered proteins can undergo the transition between order/disorder when 
they bind with their partners even though they lack the secondary and tertiary structures in their native states. The structure of full-length TDP-43 protein has not been determined by traditional methods such as NMR or X-ray crystallography. The reported NMR and crystallography structures are for parts of protein that represent the RRM domains and the $\mathrm{N}$-terminal region. Recently, a computational composite predicted structure of TDP-43 full-length protein was presented based on NMR structure of RRM (2cqg, aa 81-191), RRM2 (1wf0, aa 186-273) 2N4P (aa1-77), and 2N2C (aa 307-349) as shown in figure 2. In 2010, Chen et.al attempted to explore the physical characteristics and conformational properties of the C-terminal domain by synthesizing four peptide fragments D1 (287-322), D2 (321-353), D3 (351-383) and D4 (381-414). Their study showed that the D1 formed the fibers and D2-D4 formed the amorphous aggregates further suggesting that the D1 plays an important role in aggregate formatio $\mathrm{n}^{41}$. In a recent study conducted by Shimonaka et.al. (2016), amino acid sequence of TDP-43 that is necessary for aggregation was identified. The result showed that the glycine rich region, residues $274-313$ is necessary for the aggregate formation. In addition, it was reported that the region 274-353 is necessary for the conversion of TDP-43 into the amyloid-like fibrils and the peptide fibrils had a seeding ability. Several research groups have carried out studies to identify the amino acid sequences of TDP-43 responsible for the aggregate formation (Figure 4). Although Cterminal domain plays an important role in protein aggregation, $\mathrm{N}$-terminal domain (residues1-77) has been found to play a role in the recruitment of TDP-43 monomers into the aggregation process. In a study carried out by Kametani et.al. (2016), novel cleavage sites in TDP-43 were reported suggesting the $\mathrm{N}$-terminal half have the major cleavage sites ${ }^{42}$. Recently, more studies have been reported on the role of N-terminal domain of TDP-43 
protein. In a study by Zhang et.al. (2013), the role of residues 1-10 in N-terminus was explored and was found to play an important role in folding of TDP-43 monomer as well as formation of TDP-43 inclusion ${ }^{43}$.

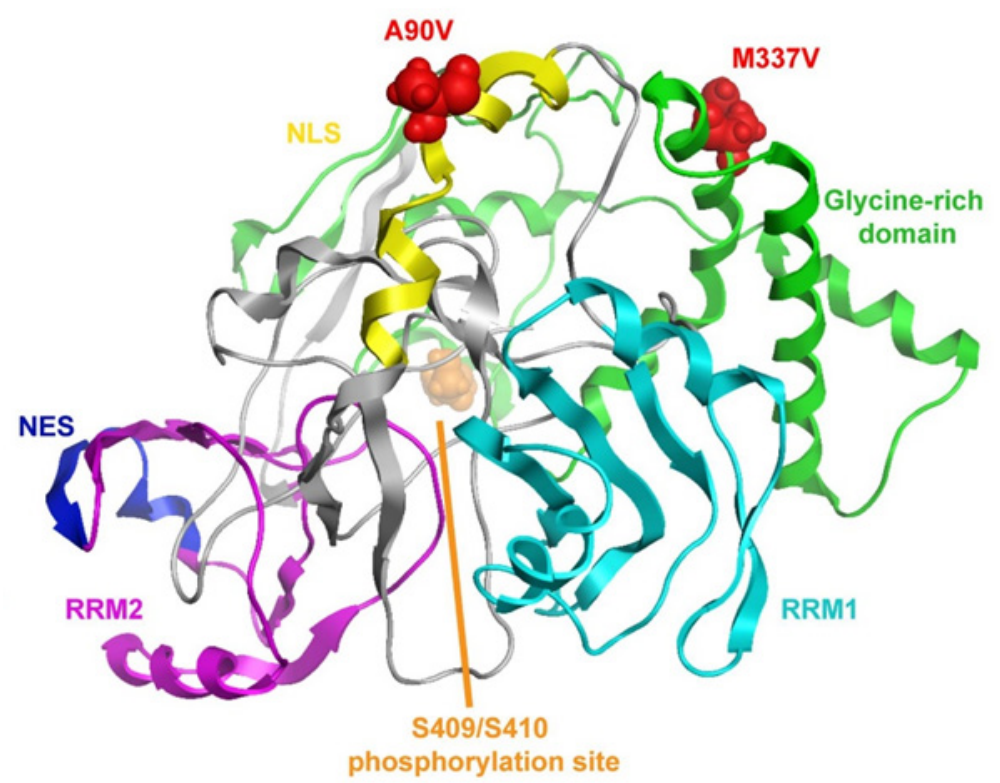

Figure 3: Schematic representation of TDP-43 structure based on the NMR structure provided. Homology modeling and loop building technique was used to generate this schematic of composite TDP-43 structure ${ }^{44}$. (Figure reprinted from Wobst J.H. et.al. (2017). Used with permission from the corresponding author)

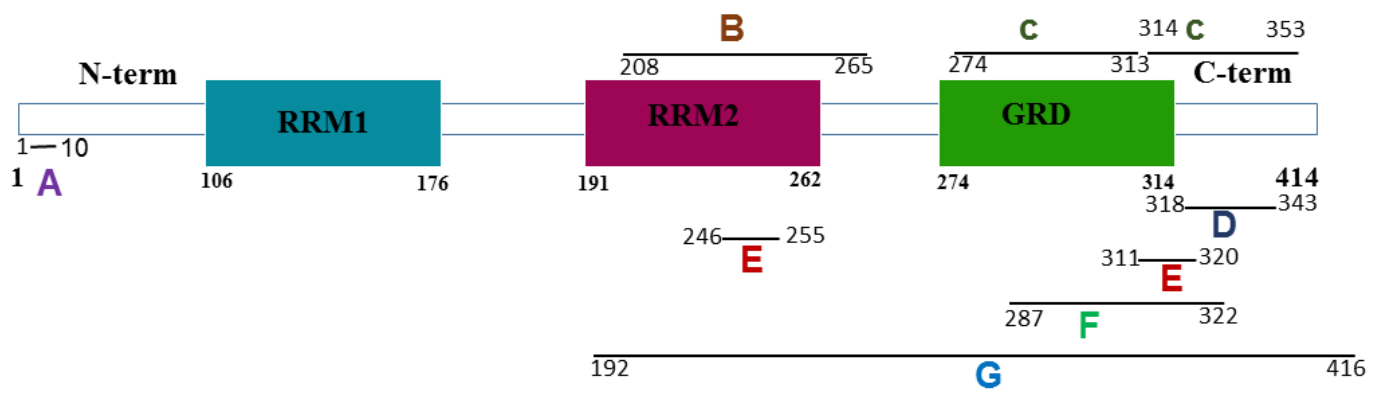


Figure 4: TDP-43 protein schematic summarizes aggregation studies carried out on the protein using truncated constructs of varying lengths ${ }^{41-43,45-48}$. A- Extreme N-terminus region is responsible for formation of TDP-43 inclusions. Zhang Y.J. et.al. (2013) Human Molecular Genetics. B - Truncated RRM2 forms amyloid negative fibrils. Wang Y. T. et.al. (2013) J. Biol. Chem. C - Residue 274-313 in glycine- rich region is essential for aggregation. Shimonako S. et.al (2016) J. Biol. Chem. D - Residue 318-343 as the amyloidogenic core is essential for TDP-43 aggregation. Jiang L. L. et.al. (2013) J. Biol. Chem. $\mathbf{E}$ - Region in RRM2 (246-255) and C-terminal (311-320) has a propensity to form aggregates and residue 311-320 plays the crucial role among both. Saini A. and Chauhan, V. S. (2011) Chembiochem. F - Residue 287-322 formed fibers whereas other residues taken under study were not amyloid in nature. Chen A. K. et.al. (2010) J. Am.Chem. Soc. G - Transduction of aggregates prepared from in vitro functional TDP-43 triggered the intracellular aggregation of TDP-43. Furukawa Y. et.al. (2011) J. Biol. Chem.

Advancement in the field of TDP-43 has led to identification of this particular protein in several neurodegenerative diseases. However, the exact mechanism of neurotoxicity is not clear and there are two main hypotheses: 1) supports the toxicity by toxic gain of function, and 2) the other supports the loss of critical function because of dominant negative mechanism. Both mechanisms of toxicity have been supported by several independent studies by scientists. In case of a gain-of-toxic function, two possible pathways are followed, first the overexpression of mutant in gene leads to disease phenotype, in which expression of normal phenotype (wild type) does not cause the disease. In the second pathway, one of the normal functions is increased in a mutant gene to greater amount leading to the toxicity. In this situation, not only the mutant but also overexpression of wild 
type gene will eventually lead to disease phenotype. A loss of function involves gene knockout or knockdown. A dominant-negative mechanism is the condition where the wildtype allele function is affected by mutant allele, as the mutant allele will not be functional and inhibits the function of wild type. Studies suggests that the overexpression of this protein leads to toxicity. On the other hand, deletion of TDP-43 has been reported to be embryonically lethal. Furthermore, the absence of cytoplasmic TDP-43 aggregates was reported to develop disease in case of TDP-43 mutant transgenic mice suggesting that the loss of TDP-43 function contributes to its pathogenesis. Also, in the study by Feiguin et.al., 2009 the in vivo neurodegeneration was observed when the TDP-43 was depleted from neuron. Considering the aggregates of TDP-43, the answer remains unclear whether these aggregates have toxic or beneficial role. Studies suggest the direct toxicity of these aggregates while study carried out in Drosophila model suggests protective role of these aggregates in early stage of disease. TDP-43 has property of self aggregation and can sequester other proteins into its aggregates. However, how this process occurs and what factors play role is yet to be determined. As a consequence of TDP-43 aggregates abnormal function of protein such as disruption of mRNA transport, altered mRNA splicing, problem

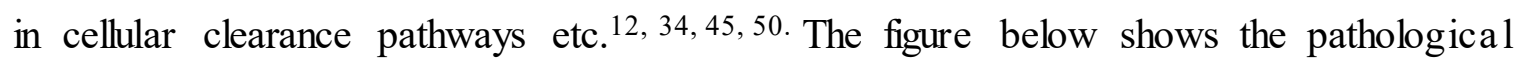
mechanism involved in neurodegeneration. 


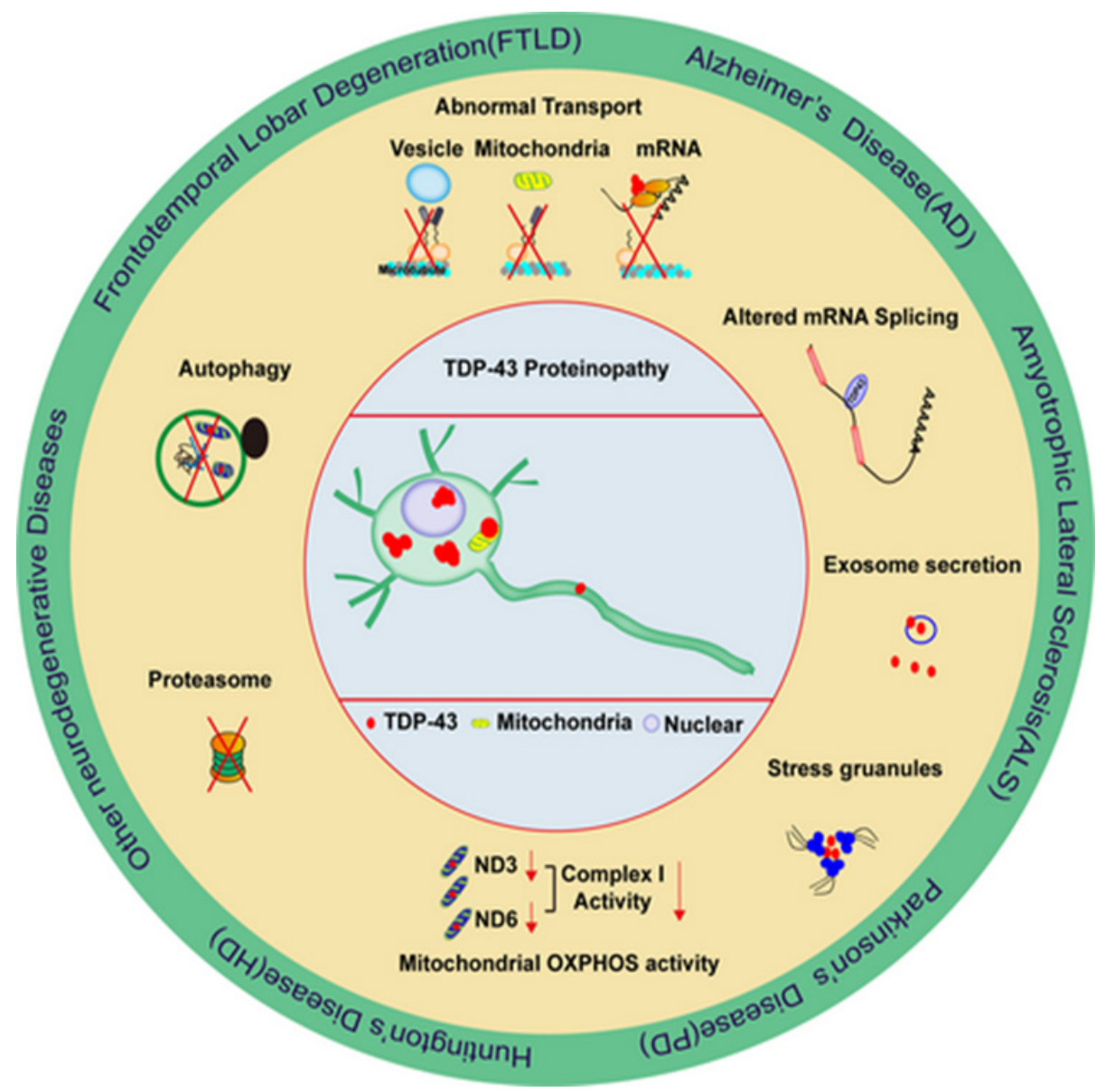

Figure 5: Pathological mechanism involved in TDP-43 neurodegeneration ${ }^{34}$. TDP-43 is involved in several neurodegenerative diseases like ALS, AD, HD etc. Under such conditions, the normal functions of TDP-43 is altered and cellular clearance mechanism is also affected. (Figure reprinted from Ratti, A et.al. Used with permission from the publisher).

The above model underscores the diverse role TDP-43 plays in health as well as disease process. To better understand how these diverse functions maybe mediated by this protein we need to better understand its biochemical and biophysical properties. For carrying out characterization of physicochemical properties of TDP-43 protein we need pure protein 
and preferably from a eukaryotic source. Biochemical and biophysical work reported on TDP-43 protein so far have been from protein expressed and purified using prokaryotic source such as bacteria. Furthermore, most studies are on truncated version of protein as indicated in figure 4. Except for computationally generated full-length structure of TDP43 protein (Figure 3), not much structural information is available on the full-length protein. Though, a substantial amount of work has been reported on TDP-43 protein, almost all of them involve the use of prokaryotic expression system. Therefore, to better understand the biochemical and biophysical properties of full-length TDP-43 protein and its implication for health and disease we need pure protein from eukaryotic source. Protein from eukaryotic source will have all-natural posttranslational modifications, that is expected in an eukaryotic protein and will be better suited to understand the disease process. TDP-43 protein has six cysteine residues and studies so far have not clarified if there are any inter-chain or intra-chain disulfide bonds (or not) in the protein. Knowing the disulfide status of a protein will help to better understand proteins biophysical properties including role disulfide bonds (or sulfhydryl residues) play in protein aggregation and/or toxicity. Hence, in this study we first standardized the protocol for purification of TDP-43 full-length protein and C-terminal construct using the eukaryotic expression system. This will help us characterize biochemical and biophysical properties of full-length protein that may be different than the properties observed for truncated versions of the protein. Purification of TDP-43 has been challenging because it aggregates when overexpressed due to presence of intrinsically disordered region. We also carried out some preliminary studies on the effect of reducing agents on TDP-43 full-length protein aggregation and how it modulates the nature of aggregates. 


\subsection{Materials and Methods:}

\section{Purification of TDP-43 protein:}

a) Preparation of expression constructs, production of recombinant baculovirus The Baculovirus particles were generated as reported previously (Hayward et al., 2002) with slight modification. Briefly, the recombinant Baculovirus were generated by transfecting the recombinant bacmid DNA into sf21 cells using the transfection reagent Cellfectin II (Invitrogen). The transfection was carried out in a 6-well plate seeded at $8 \mathrm{X}$ $10^{5}$ cells/well. $1 \mu \mathrm{g}$ of recombinant DNA and $8 \mu \mathrm{l}$ of Cellfectin II reagent were mixed with Grace's medium, unsupplemented (without antibiotics and serum) separately in equal volume. Both are combined and incubated for $30 \mathrm{~min}$. at room temperature. The mix was added dropwise onto the sf 21 cells and incubated at $27^{\circ} \mathrm{C}$ for 5 hours. The transfection medium was removed and replace with $2 \mathrm{ml}$ of complete growth medium (Grace's Insect medium, supplemented $+10 \%$ FBS). The cells were incubated at $27{ }^{\circ} \mathrm{C}$ for 72 hours or more until the signs of viral infection was noticed. The cells and media were collected and centrifuged at $1000 \mathrm{~g} / 5 \mathrm{~min}$. The supernatant containing the virus particles was stored at 4 ${ }^{\circ} \mathrm{C}$ and cell pellet at $-20^{\circ} \mathrm{C}$.

b) Cellular localization of recombinant TDP-43 in insect cells

The cellular localization of expressed TDP-43 protein was confirmed using immunohisto chemistry. First, the High-five cells were cultured in a 6 well plates containing glass cover slips and incubated overnight at $27^{\circ} \mathrm{C}$. Once the cells were nearly $50 \%$ confluent, infection with baculovirus generated for recombinant TDP-43 was performed and cells were incubated for 24,48 and $72 \mathrm{hr}$ at $27^{\circ} \mathrm{C}$. Fixation was carried out for 15 minutes at the end of each time point. For this, 3\% paraformaldehyde in $0.1 \mathrm{M}$ Pipes buffer, 
pH 6.9 was used. Cells were permeabilized by using $0.1 \%$ triton-X-100 in pipes buffer for $15 \mathrm{~min}$ and rinsed with pipes buffer for 3 times of $5 \mathrm{~min}$ each. To prevent non-specific binding of antibodies, cells were incubated with $1 \%$ of BSA in $0.1 \mathrm{M}$ pipes buffer and washed with pipes buffer three times of 5 min each. Cells were incubated with either antiTDP-43 (C-term) or anti-TDP-43 (N-term) antibodies or without any primary antibodies (negative control) in $1 \% \mathrm{BSA}$ in $0.1 \mathrm{M}$ pipes buffer for $1 \mathrm{hr}$ at room temperature. Excess antibodies were removed by rinsing with pipes buffer for $5 \mathrm{~min}$ and repeated for 3 times. The cells were further incubated with anti-rabbit secondary antibodies conjugated with Alexa Fluor 488 (Invitrogen, Cat\# A11034) for $30 \mathrm{~min}$ at room temperature. Excess antibodies were rinsed with pipes buffer for 5 min and repeated for 4 times. Mounting media with DAPI was used to mount the coverslips on the glass slide. Finally, the images were acquired using the EVOS fluorescence microscope using DAPI and GFP light cubes. c) Expression and purification of TDP-43 full length protein in High five cells:

High five cells were grown in Express Five Serum free media (externally supplemented with $20 \mathrm{mM}$ L-Glutamine and $1 \mathrm{X}$ antibiotic antimycotic) at $27^{\circ} \mathrm{C}$ with a constant speed of impellor at 95rpm. The cells in suspension culture were infected with viral stock at a log phase density of 1.0-1.5 X $10^{6}$ cells $/ \mathrm{ml}$. After $48-72 \mathrm{~h}$ of infection, the cells were harvested at the centrifugation speed of $1000 \mathrm{~g}$ for 10 minutes. A small portion of pellet was tested for protein expression and remaining was stored at $-80^{\circ} \mathrm{C}$.

The purification of full length TDP-43 was carried out using the protocol published by V.Lee et.al 2015 with slight modification ${ }^{51}$. The cell pellet was extracted in RIPA buffer (50mM Tris $\mathrm{pH} 8.0,150 \mathrm{mM} \mathrm{NaCl}, 1 \%$ Triton $\mathrm{X}-100,5 \mathrm{mM}$ EDTA, $0.5 \%$ sodium 
deoxycholate, $0.1 \%$ SDS, $1 \mathrm{mM}$ phenylmethylsulfonyl fluoride, and phosphate inhibitors

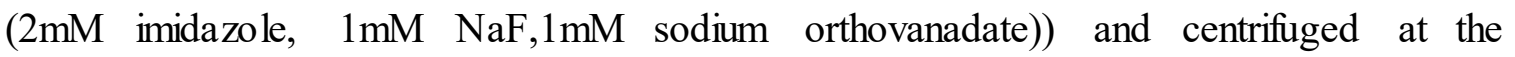
centrifugation speed of $19000 \mathrm{rpm}(42000 \mathrm{~g})$ at $40 \mathrm{C}$ for 30 minutes. Then, the insoluble pellet was re-extracted in RIPA buffer at same speed and the resulting insoluble fraction was extracted in the urea buffer $(30 \mathrm{mM}$ Tris $\mathrm{pH} 8.5,7 \mathrm{M}$ urea, $2 \mathrm{M}$ Thiourea and $4 \%$ CHAPS) at the centrifugation speed of $42000 \mathrm{~g}$ at $25^{\circ} \mathrm{C}$. The soluble fraction was then subjected to the purification by Ni-NTA affinity chromatography. The column was equilibrated with equilibration buffer (30mM Tris $\mathrm{pH} 8.5,7 \mathrm{M}$ urea, 2M Thiourea, 4\% CHAPS containing $10 \mathrm{mM}$ imidazole). The unbound proteins were washed by increasing the imidazole concentration to $50 \mathrm{mM}$ and decreasing the buffer component by $25 \%$. Finally, the protein was eluted in $30 \mathrm{mM}$ Tris $\mathrm{pH} 8.5,300 \mathrm{mM} \mathrm{NaCl}, 500 \mathrm{mM}$ Imidazole. The eluted fractions containing the TDP-43 protein were identified by SDS-PAGE and western blot. The fractions were pooled, concentrated, and stored at $-80^{\circ} \mathrm{C}$.

\section{SDS PAGE and Western blot:}

Protein estimation was done by using BCA (Bicinchoninic acid) assay. Samples for SDS PAGE were prepared by incubating the desired concentration of protein with 3X SDS sample loading buffer for 5 mins in a boiling water bath. The proteins were then separated in $12 \%$ SDS polyacrylamide gel by running the gel at $80 \mathrm{~V}$ for 3 hours in a running buffer (25 mM Tris (pH 8.3), $192 \mathrm{mM}$ glycine, and 0.1\% SDS). The gels after the run were stained

in R-250 stain ( $0.1 \%$ Coomassie Blue R250, 50\% methanol, 10\% glacial acetic acid) overnight and destained in fast and slow destaining solution. Finally, the image was captured using an Epson Scan. 
After the completion of 3 hours of run, the gel was electro blotted into PVDF membrane for western transfer. The transfer was carried out at $100 \mathrm{~V}$ for 1 hour. The membrane was blocked in 5\% blocking solution for 1 hour at $4^{0} \mathrm{C}$ shaker and incubated with primary antibody (1:4000) for overnight. Next day, the membrane was incubated with secondary antibody for 2 hours in Goat anti-rabbit IgG-HRP (1:10000). Finally, the membrane was imaged using the Gel Doc System.

\section{Reducing and Non-reducing SDS PAGE:}

Reduced sample was prepared by adding 5\% beta-mercaptoethanol while non-reduced sample was incubated with $1 \mathrm{mM}$ Iodoacetamide for 2 hours at room temperature. The reaction for non-reducing sample was terminated by adding SDS sample buffer without

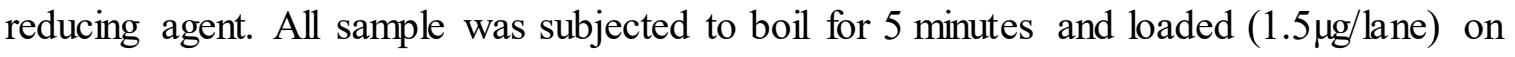
gel. The gel was run for 3 hours at $80 \mathrm{~V}$ in a running buffer $(25 \mathrm{mM}$ Tris $(\mathrm{pH} 8.3), 192 \mathrm{mM}$ glycine, and $0.1 \%$ SDS). The gels after the run were stained in R-250 stain $(0.1 \%$ Coomassie Blue R250, 50\% methanol, 10\% glacial acetic acid) overnight and destained in fast and slow destaining solution. Finally, the image was captured using an Epson Scan.

\section{Field Emission Scanning Electron Microscopy}

The morphology of the aggregates was studied using Hitachi S-4700 field-emission scanning electron microscope (FESEM). For FSEM imaging protein $(1 \mu \mathrm{M})$ was incubated in buffer $(30 \mathrm{mM}$ HEPES buffer $\mathrm{pH} 7.2+150 \mathrm{mM} \mathrm{NaCl})$ for $0 \mathrm{~h}, 1 \mathrm{~h}$, $6 \mathrm{~h}$ and $48 \mathrm{~h}$ at $37^{\circ} \mathrm{C}$ under reducing environment i.e. at different concentration of TCEP $(0,1 \mathrm{mM}$ and $10 \mathrm{mM})$. After the incubation, samples were washed using Millipore Amicon Ultra centrifugal filters (3000 Da - $5000 \mathrm{Da}$ cut off) to remove the impurities. The samples were allowed to dry at 
room temperature after aliquoting on Scanning electron microscope (SEM) stubs. The samples were coated with platinum on the SEM stubs at a thickness of 10nm using a sputter coater. Finally, the samples were imaged at the acceleration voltage of $5 \mathrm{kV}^{52}$.

\subsection{Results:}

We expressed and purified TDP-43 protein using the eukaryotic baculovirus expression system. Among the different insect cell lines like Sf21, High-five and Sf9, we choose Highfive cells because of its well-known expression of the secreted protein. As compared to $\mathrm{S} 9$, it expresses 5-10-fold higher secreted proteins. As shown in figure 6, we purchased different constructs of TDP-43 from Addgene. These constructs were 28206 consisting of full-length gene (1-414 amino acid residues and 1245 bp), 28207 which does not contain the N-terminal region of TDP-43 and consist of 86-414 amino acid residues and code for 996bp of TDP-43. 29209 is the region without the C-terminal domain and consist of 1-314 amino acid residues and codes for $948 \mathrm{bp}$ and lastly 28198 is the region containing glycine rich domain and C-terminal domain i.e. from 257-414 amino acid residues coding $471 \mathrm{bp}$. The constructs thus obtained were confirmed by Polymerase Chain reaction (PCR) using the specific primers that were designed to subclone into insect expression vector. As shown in Figure 5, we did observe the gene inserts at the designated bp. In this study we choose to use insect expression vector, pFastBac/HBM-TOPO (Figure 6). This is due to the fact that it has eukaryotic expression system, Honey bee mellitus secretory signal, polyhedrin promoter $(\mathrm{PPH})$ and 6XHis-tag at the C-terminal. This vector matched our focus of study, we aimed to express our protein in eukaryotic system with secreted overexpression and purify our protein through affinity chromatography. The positive recombinant 
pFastBac/HBM-TOPO constructs were transformed into DH10Bac E.coli cells for transposition into the bacmid DNA. The transformation procedure was followed as per the Invitrogen user manual (Invitrogen, part\# A11341) on Bac-to-Bac HBM-TOPO secreted expression system. Further, the positive bacmids were isolated using PureLink HiPure Plasmid DNA Miniprep Kit (Invitrogen) for high quality bacmid DNA. The bacmid DNA concentration was estimated by UV absorbance at $260 \mathrm{~nm}$ using Epoch Take3 microvolume plate (Bio-Tek Instruments, Inc.) and stored in aliquots at $-20{ }^{\circ} \mathrm{C}$ to avoid freezethaw cycles. The recombinant Baculovirus was generated by transfecting the recombinant bacmid DNA into sf21 cells using the transfection reagent Cellfectin II (Invitrogen). The supernatant containing the virus particles was stored at $4{ }^{\circ} \mathrm{C}$ and cell pellet at $-20{ }^{\circ} \mathrm{C}$. The outline for generation of recombinant bacmid followed by production of recombinant Baculovirus is represented in Figure 6.

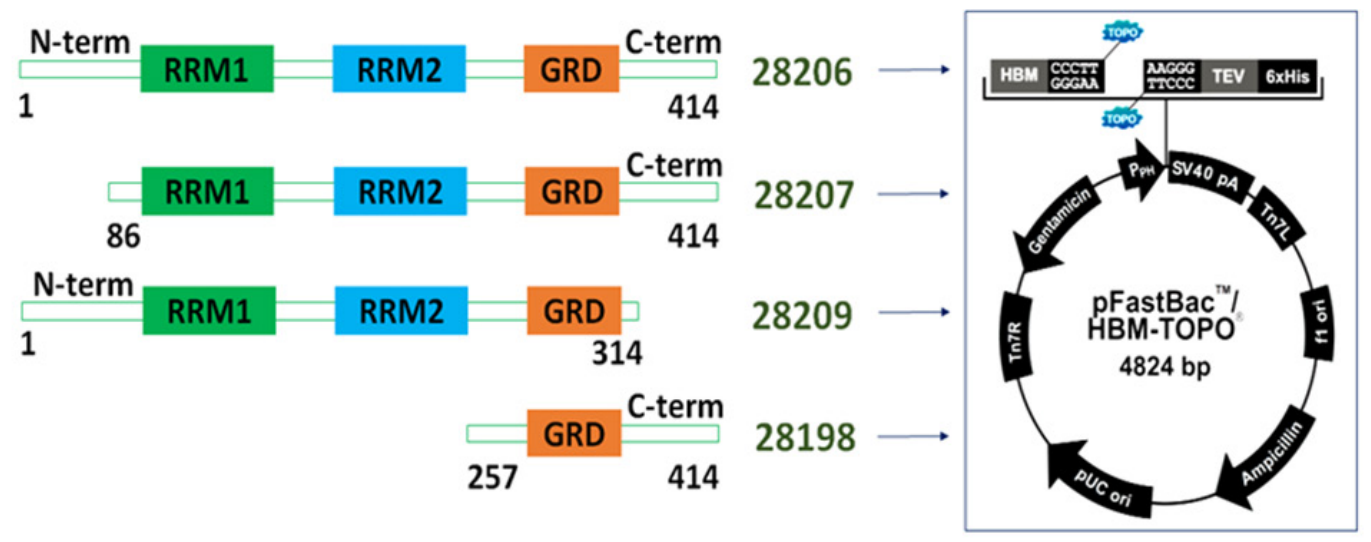

Figure 6: Different constructs of TDP-43 and vector used for sub-cloning. The differe nt constructs of TDP-43 namely 28206 (1-414 amino acid residues), 28207(86-414 amino acid residues), 29209 (1-314 amino acid residues) 28198 (257-414 amino acid residues) 
were purchased from Addgene and $\mathrm{pFastBac/HBM-TOPO}$ insect expression vector was used for sub-cloning of these constructs.

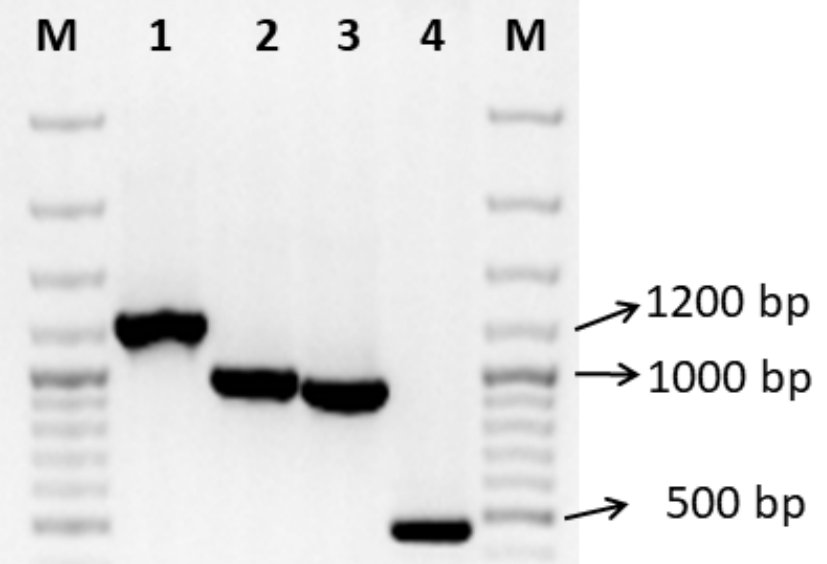

Figure 7: Verification of TDP-43 constructs obtained from addgene by PCR. Lane M: Marker, lane 1: TDP43-28206 (insert size 1245 bp), lane 2: TDP43-28207 (996 bp), lane 3: TDP43-28209 (948 bp) and lane 4: TDP43-28198 (471 bp). (The cloning work was carried out by Dr. Ashutosh Tiwari and Dr. Jagadeesh Janjanam and the figure is placed with their permission). 

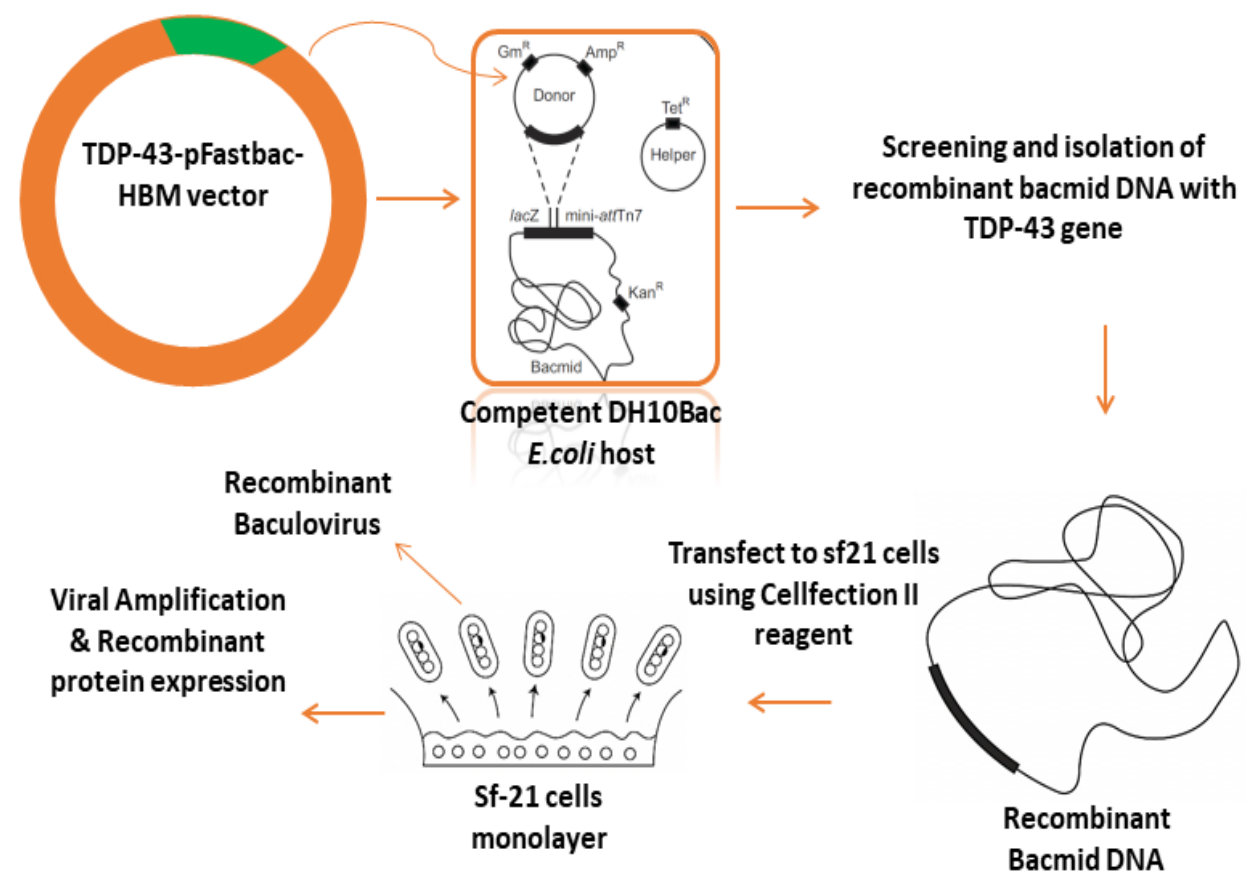

Figure 8: Schematic representation for the production of recombinant Baculovirus (with TDP-43 genes) using sf-21 insect cells (source Invitrogen user manual, part \# A11341).

Cellular localization of recombinant TDP-43 protein in insect cell was confirmed by immunocytostaining. For this purpose, we used High-five cells and proceeded as described in material and method section for immunocytostaining. As previously stated, high-five cells are well known for more secretory protein production when compared to Sf9 and even Sf2 1 cells ${ }^{56}$. As shown in figure 9 and 10 , we did not observe any signal for Control cells i.e. High-five cells without any viral stock under GFP channel using both antibodies specific to $\mathrm{N}$ - and C-terminal. However, our immunocytostaining data for High-five cells using anti-TDP-43 antibodies specific to N-terminal (Figure 10) or C-terminal (Figure 9) sequences shows that the protein is stuck in the membrane than being a secreted in a media which can be seen under GFP channel. 
With
C-term
antibodies

Controluninfection $60 \mathrm{X}$
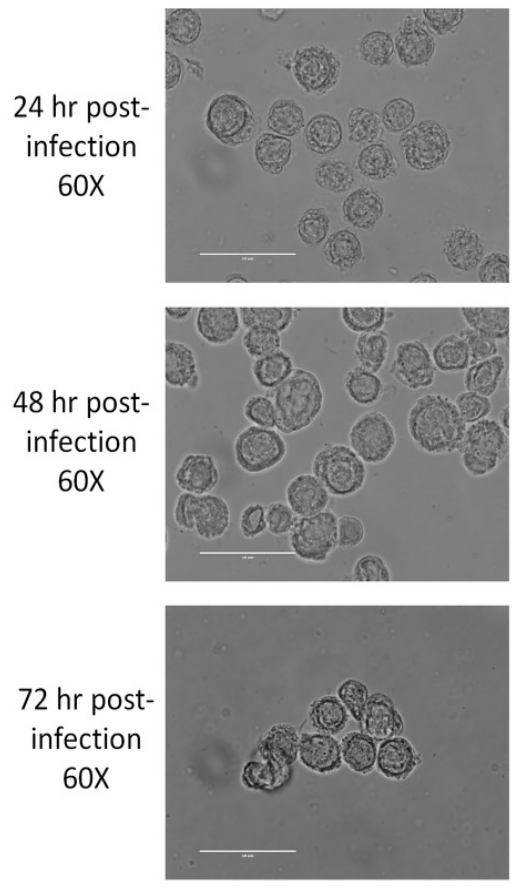

DAPI
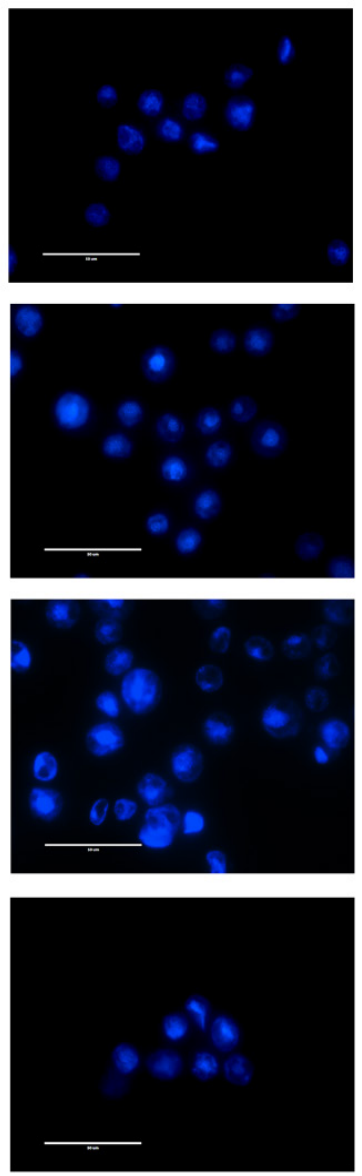

GFP_Anti-TDP-43 Ab
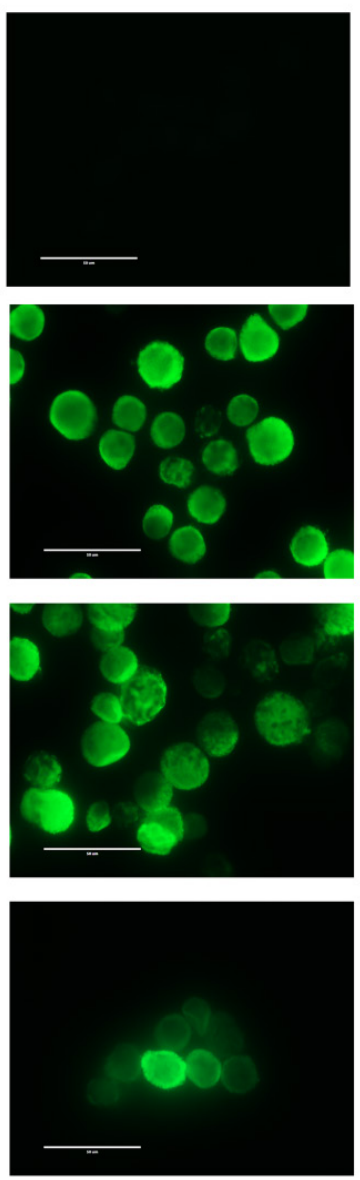

Figure 9: Immuncytostaining of high-five cells after infecting with TDP-43 viral stock. The recombinant expression of TDP-43 was detected using anti-TDP-43 antibodies specific to C-terminal 


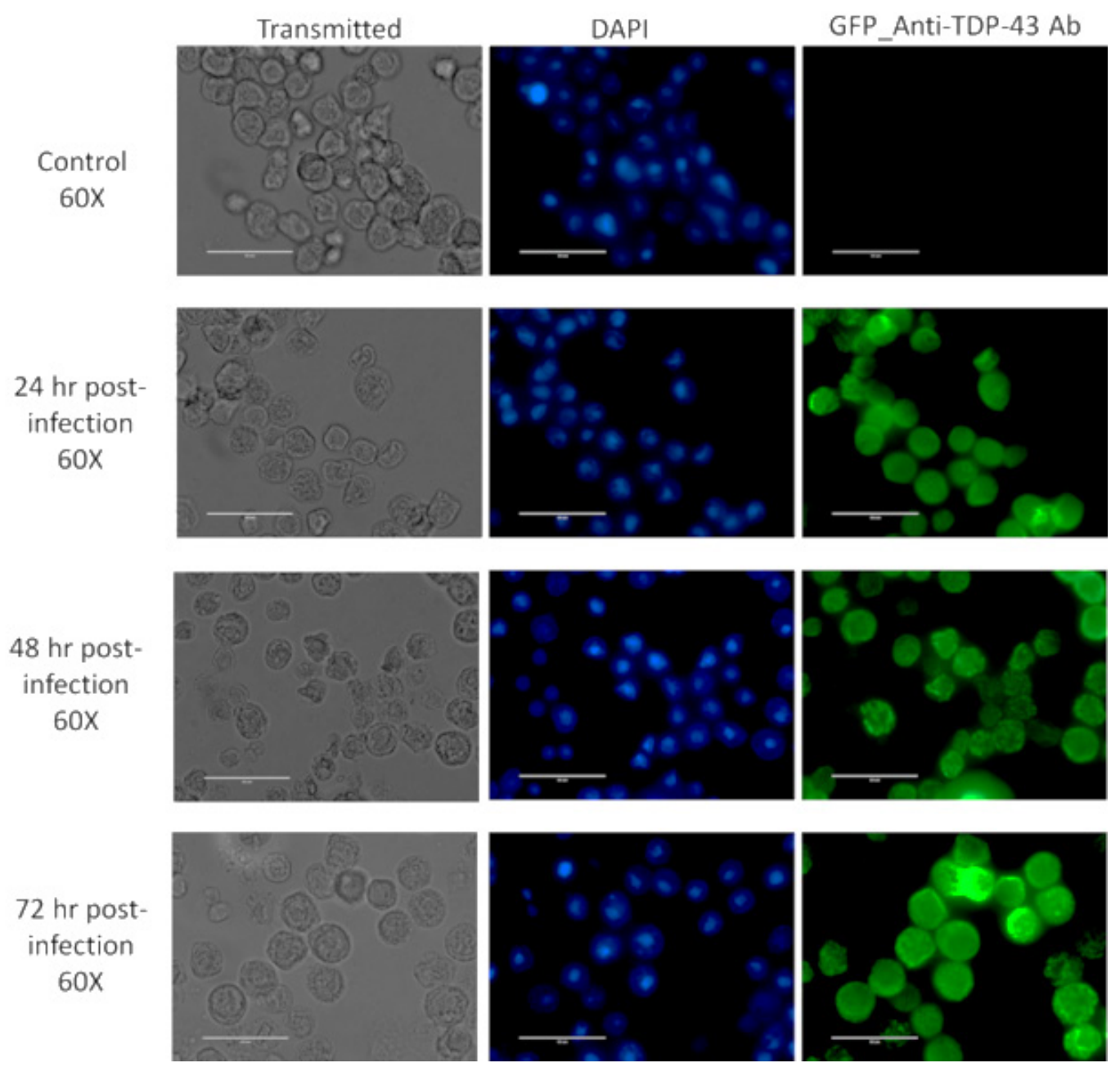

Figure 10: Immuncytostaining of high-five cells after infecting with TDP-43 viral stock. The recombinant expression of TDP-43 was detected using anti-TDP-43 antibodies specific to $\mathrm{N}$-term antibodies.

Purification of protein was most challenging and we employed different combination of techniques to purify our protein. Among these methods, we applied different percentage of ammonium sulfate, detergent, and denaturing agents. Specific details on use of different purification techniques and data are provided in supporting information for chapter 3. First, I will discuss the results for purification of full-length TDP-43 protein. Before proceeding 
to the purification step, it is important to check the expression level of protein to verify if our protein of interest is expressed or not. For that we checked each batch of our harvested cell pellets before carrying out the purification. This was accomplished by cell lysis followed by protein estimation by $\mathrm{BCA}$ assay and running different concentration of lysates in gel and carrying out western blot using anti-TDP-43 antibodies. As represented in figure S1, contro//uninfected cell $(\Phi)$ and infected cell (I) lysates were loaded at different concentration $(5,10,20$ and $30 \mu \mathrm{g} / \mathrm{lane})$ in SDS PAGE and Western blot. As shown in figure S1, the western blot data showed the presence of protein band $(\sim 50 \mathrm{kDa})$ only in infected cell lysate but not for control lane at all concentrations loaded. The SDS PAGE showed the multiple bands at all concentration but represents the identical loading for control and infected cell lysates. Further, SDS-PAGE confirms the need of further purification.

Next, we proceeded to enrichment of protein by using ammonium sulfate. As represented in the flow chart figure S2, different percentage of ammonium sulfate was added to protein. We used $30 \%, 50 \%$ and $70 \%$ saturated ammonium sulfate solutions. As presented in figures S3-S4, we observed band for TDP-43 protein in Western blot for lysates supernatant (S) or pellet (P) fractions represented as S0 (total lysate after lysis) ,S30 (lysate after 30\% ammonium sulfate precipitation) and P0 (pellet after cell lysis), and P30 (pellet after 30\% ammonium sulfate precipitation). Only a faint band was observed for P50 and no band was observed for S50, S70 and P70. Ni-NTA purifications of P30 fraction showed band for TDP-43 eluted in different fractions but was not a clean single band as observed by SDS-PAGE (Figure S5-S6). In addition, significant amount of protein was also observed in bound flow through and wash flow through obtained from column. Analyzing 90 
the results from ammonium sulfate precipitation, the protein did not completely precipitate in different percentage of ammonium sulfate solutions as shown by presence of protein in S0, S30, and P30.

Our next step was solubilizing the protein using detergent. For that purpose, we used SDS. The SDS PAGE and Western blot data for detergent soluble fraction (Figure S14) represented the band for TDP-43 full length protein in western blot but not in SDS-PAGE. The higher amount of protein was observed with increasing concentration but some additional faint bands were also observed with increasing protein concentration. We saved the detergent insoluble fraction and ran them on gel loading different volumes $(1,4,6,10$, 14,16 and 20ul) represented as DI2, DI4, DI6, DI10, DI14, DI 16, DI20 and ran detergent soluble $10 \mu \mathrm{g} / \mathrm{lane}$ for comparison of band with detergent insoluble fraction (Figure S10). We observed the thick band for our protein of interest $\sim 50 \mathrm{kDa}$ for all volumes loaded in SDS-PAGE but there were several other bands observed. Also, the thickness of band

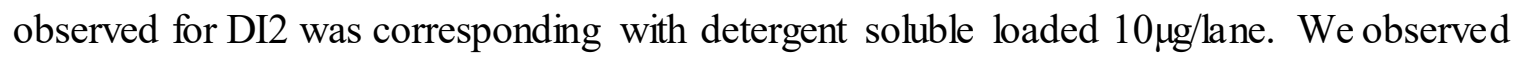
that our protein band is present but contains impurities and most of the protein is in detergent insoluble fraction.

After several rounds of trials and errors, we came up with the procedure that uses combination of salt, detergent and denaturant for solubilizing protein and purifying it to homogeneity. Using urea buffer combination helped us to purify protein with $>95 \%$ purity (figure 11). The purity of protein in each step during this procedure is shown in Figure S11. As shown in figure, the control lysate, infected cell lysate , RIPA re-extracted fraction did not show any band in western blot. However, a clear band is observed for urea soluble and 
NI-NTA purified fraction. Also, the SDS-PAGE corresponds the purified protein in Gel and Western blot. A clear depiction of urea soluble and purified protein is shown in figure 11. Since, the same concentration of protein was loaded in gel and western blot i.e. infected lysate $(10 \mu \mathrm{g} / \mathrm{lane})$, urea soluble fraction $(5 \mu \mathrm{g} / \mathrm{lane})$, Purified protein $(2 \mu \mathrm{g} / \mathrm{lane})$, the level and purity of protein is clearly detected. A significant amount of protein is seen in urea soluble fraction, but some impurities was observed but after NI-NTA chromatography, the eluted protein was found to be highly pure.

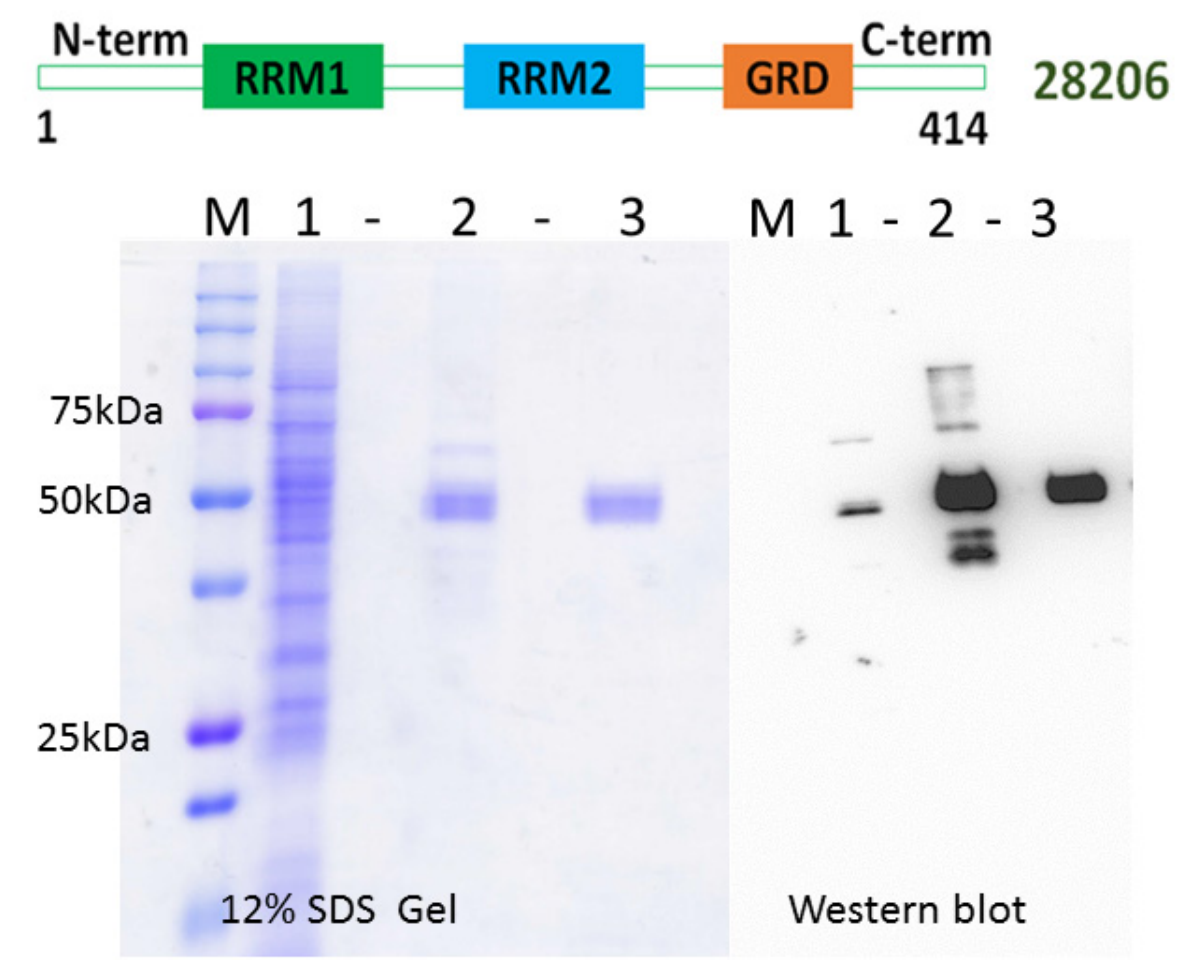

Figure 11: Reducing SDS PAGE and Western blot showing the purification of TDP-43 full length protein. Lane 1 (infected lysate, $10 \mu \mathrm{g} / \mathrm{lane}$ ), Lane 2 (urea soluble fraction, 5 $\mu \mathrm{g} / \mathrm{lane}$ ), Lane 3 (Purified protein, $2 \mu \mathrm{g} / \mathrm{lane}$ ) was loaded on 12\% SDS PAGE. The gels 
were run for 3 hours. For Western blot, the blot was incubated with Anti-TDP-43 (Nterminal region) produced antibody $(1: 4000)$ and Goat anti-rabbit IgG-HRP (1:10000).

Aggregation of TDP-43 full- length protein was observed using FESEM. We did not observe any aggregates for TDP-43 full length protein fresh samples suggesting the protein is soluble. However, the aggregates were observed after $1 \mathrm{~h}$ of incubation for all samples incubated in presence and absence of TCEP and same phenomenon was observed for $6 \mathrm{~h}$ and $48 \mathrm{~h}$ of incubation (Figure 12). The shape of aggregates were different in presence and absence of TCEP but all were amorphous in nature. Increasing the concentration of TCEP from $1 \mathrm{mM}$ to $10 \mathrm{mM}$ also resulted into amorphous aggregates (Figure S11-S14).

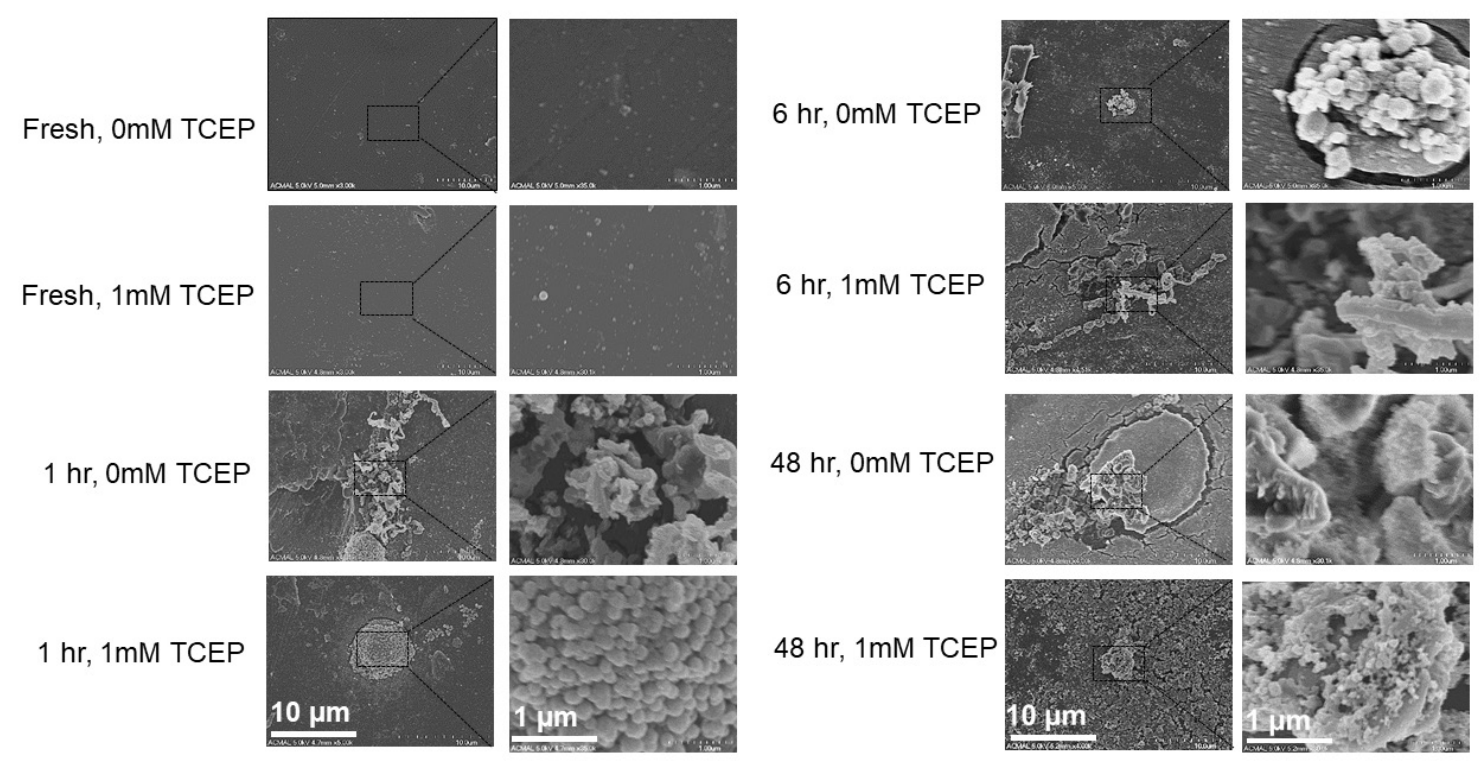

Figure 12: FESEM images of TDP-43 full-length aggregates in presence and absence of TCEP. TDP-43 protein $(1 \mu \mathrm{M})$ was incubated in presence or absence of TCEP $(0,1 \mathrm{mM})$ for $0,1,6$ and $48 \mathrm{~h}$ at $37^{\circ} \mathrm{C}$. Protein aggregates were observed under FESEM. Zoomed in image 
for the area in the box is represented on its side. Scale bar $=10 \mu \mathrm{M}$ and $1 \mu \mathrm{M}$ from left to right.

In order to investigate the status of disulfide bond in TDP-43 full- length protein, we first ran the protein samples under reduced and non-reduced conditions. Our data showed almost similar pattern in 12\% SDS Gel for TDP-43 full- length protein (Figure 14). Since, we did not observe any difference in mobility and shape of protein, we need to investigate further to understand the disulfide status of the protein.

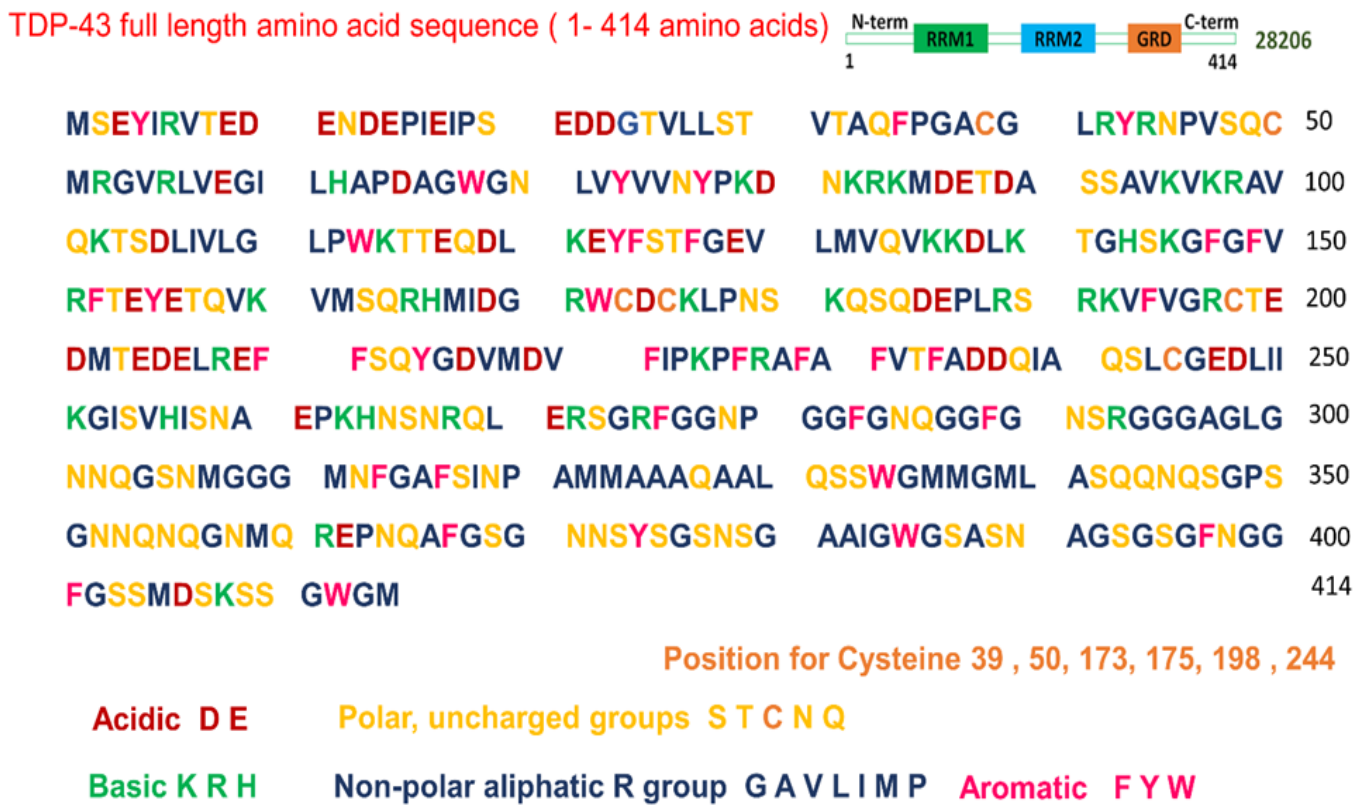

Figure 13: Amino acid sequence of TDP-43 full-length (1-414) protein representing acidic, basic, aromatic, polar uncharged, non-polar aliphatic groups and cysteine residues. Six cysteine residues are located in TDP-43 at the positions 39, 50, 173, 175, 198 and 244. 


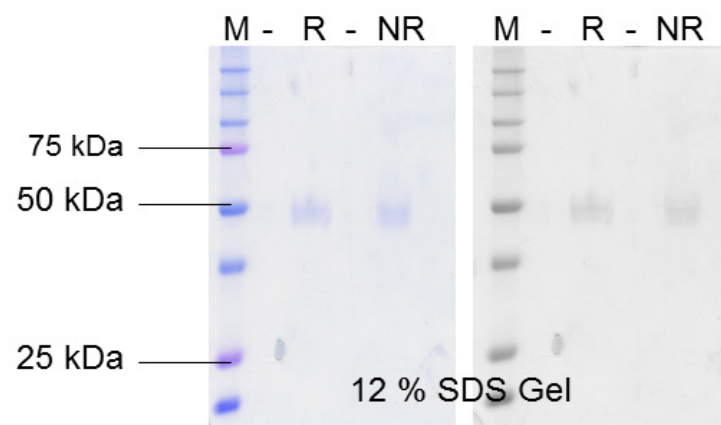

Figure 14: SDS PAGE showing TDP-43 reduced and non-reduced samples. Reduced sample (R) was treated with 5\% beta-mercaptoethanol and non-reduced sample (NR) was treated with Iodoacetamide. Protein was loaded $1.5 \mu \mathrm{g} /$ each lane and run on a $12 \%$ SDSPAGE for 3 hours.

Purification of C-terminal construct (28198) was carried out in a way similar to as described for full-length protein. Like, full-length protein, majority of protein was present in the insoluble fraction after lysis so we solubilized protein in urea buffer before purifying on a Ni-NTA chromatography column (Figure S15-S16).

\subsection{Discussion :}

Expression and purification of protein is routinely employed in lab procedure to study its biophysical, molecular and physicochemical properties. However, identifying the proper method of purification yielding pure protein of interest is very challenging. In our study, we employed different combination of methods to purify TDP-43 protein. Among these methods, we employed different percentage of ammonium sulfate, detergent, and chaotropic agents. Several studies on truncated forms of TDP-43 protein have been reported. However, information on biochemical and biophysical properties of full-length 
protein is limited due to its difficulty in expression and purification. Furthermore, studies reported are from proteins from prokaryotic source. To investigate the full-length protein and characterize its physicochemical properties along with other constructs of TDP-43, we used $\mathrm{pFastBac/HBM-TOPO}$ vector for subcloning (Figure 4), a eukaryotic vector. pFastBac/HBM-TOPO vector has many features including a secreted protein expression signal (HBM) and a 6X His-tag. This can facilitate protein purification using Ni-NTA affinity chromatography. However, in our case the purification needed several intermediate steps before purification on an affinity column. Confirmation of the constructs were performed through PCR and subcloning and viral amplification were carried out using the established protocol from Invitrogen. For production of viral stock, we used Sf21 cells because they are well suited for transfection, plaque purification, generating high-titer stocks, plaque formation, and expression of recombinant proteins. After generating the viral stock, it's important to test the cellular localization of protein. As shown in figure 3, we used high-five cells for testing the cellular localization and production of our recombinant protein. Incubation of cells with either $\mathrm{N}$ or $\mathrm{C}$-terminal antibodies resulted in signal in GFP channel and no signal in control cells. Immunocytostaining confirmed the cellular expression of our protein upto $72 \mathrm{~h}$ of post-infection of viral stock. However, we did not observe the secreted expression of protein. Instead of protein being released in the media, protein was stuck in the membrane. We employed different methods to purify the TDP-43 full length protein to get greater than $95 \%$ purity. Before applying any purification technique, it's very important to confirm the expression of the protein. For that purpose, we also tested each batch of our harvested cell pellet for its expression of TDP-43 protein and confirmed by Western blot. Presence of protein band only in infected cell lysate and 
absence in control lysate confirms our protein expression (Figure S1). After confirming the expression of protein, the bulk lysis as well as purification procedure was carried out.

We first chose to enrich our protein using ammonium sulfate for precipitation by saltingout. Precipitation of protein does not mean its denaturation, it's the condition of reduced solubility which is also related to the enhancement of protein native form stability. Ammonium sulfate is most commonly used salt in the enrichment of protein because of its high solubility in water and ability to salt-out some proteins preferentially. Therefore, initially we also employed the enrichment of TDP-43 protein using saturated solution of ammonium sulfate. As shown in figures S2-S7, different percentage of saturated ammonium sulfate solution was used, and protein mostly remained in pellet fraction at $30 \%$ ammonium sulfate concentration or higher. Affinity chromatography of pellet fraction yielded the bands on western blot but could not resolve the clear bands on the SDS-PAGE.

Detergents are the molecules carrying the amphipathic properties and can disrupt or form the hydrophobic-hydrophilic interactions among molecules. Detergents are used in research especially in biological samples for various purposes like cell lysis for the release of soluble proteins, lipids and membrane proteins solubilization, prevents nonspecific binding in affinity purification as well as in immunoassay, additives in electrophoresis. We also used SDS, anionic detergent to solubilize our full-length protein as our protein was stuck in the membrane. SDS-PAGE and Western blot data showed the protein fraction present in higher amount in insoluble fraction compared to the soluble fraction. (Figure S8S9). After several trials, we developed a purification strategy for full-length TDP-43 
protein using a combination of salt, denaturant, detergent, and affinity chromatography to get $>95 \%$ pure protein.

Urea is the chaotropic agent which is most commonly used in solubilization as well as denaturation of proteins. Apart from this urea is used for protein renaturation that has been denatured using $6 \mathrm{M}$ guanidine hydrochloride ${ }^{53-55}$. Since, most of our protein was present in pellet fraction after lysis in RIPA buffer, we used combination of buffer containing urea, thiourea and CHAPS to solubilize our protein present in pellet. Using this method, we were able to achieve $>95 \%$ purity which we confirmed by gel and Western blot. (Figure 11)

Protein aggregation is well studied topic. Next, question in the field of TDP-43 is understanding the nature of aggregated form of TDP-43 protein. Different studies carried out by different groups reported on the aggregates of TDP-43 protein (Figure 4). Some studies support the nature of aggregates to be amorphous while other supports the aggregates to be amyloid in nature ${ }^{56-59}$. Since, we do not have clear picture regarding the disulfide status of the protein, we wanted to understand if full-length TDP-43 protein forms aggregates under disulfide reducing environment or not. The interesting of all is the studies reported are on the different truncated constructs of the protein and is carried out using prokaryotic source. Aggregation of TDP-43 full-length protein carried out in bacteria shows the amorphous nature. Therefore, we studied the aggregation of TDP-43 by subjecting it to different concentration of reducing agent and incubation time and observing the aggregates under microscope. Our finding suggests the TDP-43 forms aggregates under disulfide reducing environment and the nature of TDP-43 full-length aggregates are amorphous. Also, our fresh protein did not form any aggregates in absence and presence 
of $1 \mathrm{mM}$ TCEP up to $1 \mathrm{~h}$, suggesting our protein is soluble and stable and does not form aggregate under its normal condition.

TDP-43 has six cysteine residues located at position 38, 49, 172, 174, 197 and 243 (Figure 10). The possibility of formation of disulfide linked TDP-43 dimers and oligomers in vivo was reported in the study conducted by Zhang et.al. (2011). However, it was suggested that formation of aggregates does not require such modification. However, the exact disulfide status of TDP-43 has not been reported yet. It is not clear that how many disulfide bonds are there in TDP-43 protein. Disulfide bond is present in most of the proteins and it ensures the correct folding of protein under native conditions. Disulfide bonds occupy $15 \%$ of the human proteome and are present mainly in secreted protein where it makes up to $65 \%$. Next to peptide bond, the common covalent link between the amino acids in the protein is the disulfide bond. Disulfide bonds are known to decrease the entropy of the unfolded state thereby stabilizes the protein thermodynamically. The presence of disulfide bonds are found in $55 \%$ of the pathologic amyloid forming proteins providing the information about the importance of disulfide bonds in the aggregation kinetics as well as its role in the toxicity and structure of aggregates. For the proper folding of protein into its native structures, many proteins require the correct formation of disulfide bonds. Protein misfolding results when there is abnormal reduction/formation of disulfide bonds. Disulfide bonds are involved in proteins involved in neurodegeneration. Until date, the role of disulfide bonds have been studied and found to be important in three proteins involved in neurodegeneration: prion, SOD1 and Tau. The folding/misfolding pathway can thus be traced by determining the thiol-disulfide status in the protein. For example, the folding/misfolding is regulated by thiol-disulfide status in case of SOD1 $(\mathrm{Cu}, \mathrm{Zn}$ - 
superoxide dismutase). The stabilization of matured SOD1 is achieved by the formation of intramolecular disulfide bonds whereas the reduction of these disulfide bonds leads to the misfolding and aggregation of SOD1 due to a decrease in conformational stability of a protein. Therefore, the disulfide bonds play important role in familial form of amyotrophic lateral sclerosis (fALS ${ }^{60-63}$. In order to understand the disulfide status of TDP-43, we ran the samples under reduced and non-reduced conditions (Figure 14). The mobility of proteins under two conditions showed no major variation suggesting us need of further confirmation.

To compare the characteristics of TDP-43 we next purified C-terminal domain of TDP-43 using the same protocol used for full-length protein (Figure S15-S16). Purifying more of protein will help to compare the properties of full-length protein with most aggregation prone domain of TDP-43. C-terminal region is the most studied domain of TDP-43 and much of mutations known are found in this domain. Having this region for comparison will help us to compare the findings reported in prokaryotic system with our eukaryotic source and report any variations if present.

\subsection{Conclusion:}

In summary, this study describes the standardized protocol for expression and purification of TDP-43 full- length protein using eukaryotic source. The result shows the purity of protein $>95 \%$ as confirmed by gel and Western blot. Further, the nature of aggregates of full- length proteins were confirmed under reducing environment and were found to be amorphous in nature. Apart from full-length protein our purification protocol is applicable to intrinsically disordered portion of this protein, i.e. C-terminal region. 


\subsection{References:}

1. Li, Q.; Yokoshi, M.; Okada, H.; Kawahara, Y., The cleavage pattern of TDP-43 determines its rate of clearance and cytotoxicity. Nature Communications 2015, 6:6183.

2. Aaron, C.; Beaudry, G.; Parker, J.A.; Therrien M., Maple Syrup Decreases TDP-43 Proteotoxicity in a Caenorhabditis elegans Model of Amyotrophic Lateral Sclerosis (ALS). Journal of Agriculture and Food Chemistry 2016, 10.1021.

3. Strong, M.J.; Volkening, K.; Hammond, R.; Yang, W.; Strong, W.; Lantz, C.L.; Shoesmith, C., TDP43 is a human low molecular weight neurofilament (hNFL) mRNAbinding protein. MCN 2007, 35:320-327.

4. Li, W.; Jin, Y.; Prazak, L.; Hammell, M.; Dubnau, J., Transposable elements in TDP-43 Mediated neurodegenerative disorders. PLOS ONE2012,10: 1-10.

5. Nonaka, T.; Suzuki, G.; Tanaka, Y.; Kametani, F.; Hirai, S.; Okado, H.; Miyashita, T.; Saiyoe, M.; Akiyama, H.; Masai, H.; Hasegawa, M., Phosphorylation of TAR DNAbinding Protein of $43 \mathrm{kDa}$ (TDP-43) by Truncated Casein Kinase $1 \delta \delta$ Triggers Mislocalization and Accumulation of TDP-43. J. Biol.Chem 2016, 291:5473-5483.

6. Tourenne, C.L.; Polymenidou, M.; Cleveland, D.W., TDP-43 and FUS/TLS: emerging roles of RNA processing and neurodegeneration. Human Molecular Genetics 2010, 19(1): R46-R64.

7. Ayala, V.; Serrano, A.BG. et.al., Cell stress induces TD-43 pathological changes associated with ERK1/2 dysfunction: implication in ALS. Acta Neuropathol 2011, 122:259-270. 
8. Boillee, S.; Vande, V. C.; Cleveland, D.W.; ALS: a disease of motor neurons and their nonneuronal neighbors. Neuron 2006, 52:39-59.

9. Pasinelli, P.; Brown R.H., Molecular biology of amyotrophic lateral sclerosis: insights from genetics. Nat. Rev. Neurosci 2006, 7: 710-723.

10. Li, Q.; Yokoshi, M.; Okada, H.; Kawahara, Y., The cleavage pattern of TDP-43 determines its rate of clearance and cytotoxicity. Nature Communications 2015, 6:6183.

11. Huang, Y.C.; Lin, K.F.; He, R.Y.; Tu, H.P.; Koubek, J.; Hsu, Y.C.; Huang, J.J., Inhibition of TDP-43 aggregation by Nucleic Acid Binding. PLOS ONE 2013, 8(5): 111.

12. Xu, S.Z., Does a loss of TDP-43 function cause neurodegeneration? Molecular Neurodegeneration 2012, 7(27):1-10.

13. Amador, O.C.; Lin, W.L.; Ahmed, Z.; Personett, D.; Davies, P.; Duara, R.; GraffRadford N.R.; Hutton, M.L.; Dickson, D.W., TDP-43 immunoreactivity in hippocampal sclerosis and Alzheimer's disease. Ann. Neurol 2007 61: 435-445.

14. Nakashima, Y.H.; Uryu, K.; Robinson, J.; Xi, S.X.; Hurtig, H.; Duda, J.E., et al. Comorbidity of TDP-43 proteinopathy in Lewy body related diseases. Acta Neuropathol 2007 114: 221-229.

15. Wider, C.; Dickson, D.W.; Stoessl, A.J.; Tsuboi, Y.; Chapon, F.; Gutmann, L.; Lechevalier, B.; Calne, D.B.; Personett, D.A.; Hulihan, M., et al. Pallidonigral TDP-43 pathology in Perry syndrome. Parkinsonism Relat. Disord 2009, 15:281-286. 
16. Uryu, K.; Nakashima, Y.H.; Forman, M.S.; Kwong, L.K.; Clark, C.M.; Grossman, M.; Miller, B.L.; Kretzschmar, H.A.; Lee, V.M.; Trojanowski, J.Q., et al. Concomitant TAR-DNA-binding protein 43 pathology is present in Alzheimer disease and corticobasal degeneration but not in other tauopathies. J. Neuropathol. Exp. Neurol 2008, 67: 555-564.

17. Lee, B.E.; Lee, V.M.Y.; Trojanowski, J.Q., Gains or losses: molecular mechanisms of TDP-43- mediated neurodegeneration. Nature reviews 2012, 13: 38-48.

18. Buratti, E.; Baralle, F. E., Multiple roles of TDP-43 in gene expression, splicing regulation, and human disease. Frontiers in Bioscience 2008, 13: 867-878.

19. Hanson, K.A.; Kim, S.H.; Tibbetts, S.R., RNA- binding proteins in neurodegenerative diseases: TDP-43 and beyond. WIREs RNA 2012, 3: 265-285.

20. Hart, F.U.; Bracher, A.; Hart, M.H., Molecular chaperones in protein folding and proteostasis. Nature Review 2011, 10: 324-332.

21. Forman, M.S.; Trojanowski, J.Q.; Lee, V.M., Neurodegenerative diseases: a decade of discoveries paves the way for therapeutic breakthroughs. Nature Medicine 2004, 10: $1038 / 1113$

22. Soto, C., Unfolding the roles of protein misfolding in Neurodegenerative Diseases. Nature Reviews 2003, 4:49-60.

23. Hirth, F., Drosophila melanogaster in the Study of Human Neurodegeneration. CNS \& neurological Disorders -Drug Target 2010, 9: 504-523. 
24. Romano, V.; Quadri, Z.; Baralle, F.E.; Buratti, E., The structural integrity of TDP-43 $\mathrm{N}$-terminus is required for efficient aggregate entrapment and consequent loss of protein function. Prion 2015, 9:1-9.

25. Rohn, T.T., Cytoplasmic inclusions of TDP-43 in neurodegenerative diseases: A potential role for Caspases. Histol Histopathol. 2009, 24: 1081-1086.

26. Cohen, T.J.; Hwang, A.W.; Unger, T.; Trojanowski, J.Q.; Lee, V M.Y., Redox signaling directly regulates TDP-43 via cysteine oxidation and disulphide crosslinking. The EMBO Journal 2012, 31: 1241-1252.

27. Buratti, E.; Brindisi, A.; Pagani, F.; Baralle, F.E., Nuclear factor TDP-43 binds to the polymorphic TG repeats in CFTR intron 8 and causes skipping of exon 9: a functional link with disease penetrance. Am J Hum genet 2004, 74: 1322-1325.

28. Lee, B.E.; Lee, V.M.Y.; Trojanowski, J.Q., Gains or losses: molecular mechanisms of TDP-43- mediated neurodegeneration. Nature reviews 2012, 13: 38-48.

29. Ou, S.H.; Wu, F.; Harrich, D.; Garcia-Martinez, L.F.; Gaynor, R.B., Cloning and characterization of a novel cellular protein, TDP-43, that binds to human immunodeficiency virus type 1 TAR DNA sequence motifs. J Virol 1995, 69: 35843596.

30. Freibaum, B.D.; Chitta, R.K.; High, A.A.; Taylor, J.P., Global analysis of TDP-43 interacting proteins reveals strong association with RNA splicing and translation machinery. J. Proteome Res 2010, 9: 1104-1120.

31. Buratti, E.; De, Conti L.; Stuani, C.; Romano, M.; Baralle, M.; Baralle, F., Nuclear factor TDP-43 can affect selected microRNA levels. FEBS J 2010, 277: 2268-2281. 
32. Lu, Y.; Ferris, J.; Gao, F.B., Frontotemporal dementia and amyotrophic lateral sclerosis associated disease protein TDP-43 promotes dendritic branching. Mol. Brain 2009, 2: 30.

33. Moisse, K., et.al. Cytosolic TDP-43 expression following axotomy is associated with caspase 3 activation in NFL-/- mice: support for a role for TDP-43 in the physiological response to neuronal injury. Brain Res. 2009, 1296: 176-186.

34. Ratti, A.; Buratti, E., Physiological functions and pathobiology of TDP-43 and FUS /TLS protein. Journal of Neurochemistry 2016, 138 (95-111).

35. Sephton, C.F.; Good, S.K.; Atkin, S.; Dewey, C.M.; Mayer, O., et.al. TDP-43, a neuropathosignature factor, is essential for early embryonic development. J.Biol.Chem 2010, 285: 6826-6834.

36. Rohn, T.T., Cytoplasmic inclusions of TDP-43 in neurodegenerative diseases: A potential role for Caspases. Histol Histopathol. 2009, 24: 1081-1086.

37. Li, W.; Jin, Y.; Prazak, L.; Hammell, M.; Dubnau, J., Transposable elements in TDP43 Mediated neurodegenerative disorders. PLOS ONE 2012, 10: 1-10.

38. Nonaka, T.; Suzuki, G.; Tanaka, Y.; Kametani, F.; Hirai, S.; Okado, H.; Miyashita, T.; Saiyoe, M.; Akiyama, H.; Masai, H.; Hasegawa, M., Phosphorylation of TAR DNAbinding Protein of $43 \mathrm{kDa}$ (TDP-43) by Truncated Casein Kinase $1 \delta \delta$ Triggers Mislocalization and Accumulation of TDP-43. J. Biol.Chem 2016, 291:5473-5483.

39. Amador, O.C.; Lin, W.L.; Ahmed, Z.; Personett, D.; Davies, P.; Duara, R.; GraffRadford, N.R.; Hutton, M.L.; Dickson, D.W., TDP-43 immunoreactivity in hippocampal sclerosis and Alzheimer's disease. Ann. Neurol 2007, 61: 435-445. 
40. Nakashima, Y.H.; Uryu, K.; Robinson, J.; Xi, S.X.; Hurtig, H.; Duda, J.E., et al. Comorbidity of TDP-43 proteinopathy in Lewy body related diseases. Acta Neuropathol 2007, 114: 221-229.

41. Chen, A.KH., et.al. Induction of Amyloid Fibrils by the C-terminal Fragments of TDP43 in Amyotrophic Lateral Sclerosis. JACS communications 2010, 132: 1186-1187.

42. Shimonako, S.; Nonaka, T.; Suzuki, G.; Hisanaga, S.; Hasegawa M., Templated Aggregation of TAR DNA-binding Protein of $43 \mathrm{kDa}$ (TDP-43) by Seeding with TDP43 Peptide Fibrils. The Journal of Biological Chemistry 2016, 291: 8896-8907.

43. Zhang, Y.J., et.al. The dual functions of the extreme N-terminus of TDP-43 in regulating its biological activity and inclusion formation. Human Mol. Genet 2013, 22(15): 3112-3122.

44. Wobst, H.J.; Wesolowski. S.S.; Chadchankar. J.; Delsing, L.; Jacobsen, S.; Mukherjee J.; Deeb. T.Z.; Dunlop. J.; Brandon. N.J.; Moss. S.J., Cytoplasmic Relocalization of TAR DNA-Binding Protein 43 Is Not Sufficient to Reproduce Cellular Pathologies Associated with ALS In vitro. Front Mol Neurosci.2017 10:46.

45. Wang, Y.T., et.al. The truncated C-terminal RNA recognition motif of TDP-43 protein plays a key role in forming proteinaceous aggregates. J. Biol.Chem 2013, 288: 90499057.

46. Jiang L.L. et.al. Structural transformation of the amyloidogenic core region of TAR DNA binding protein of $43 \mathrm{kDa}$ (TDP-43) initiates its aggregation and cytoplasmic inclusion. J.Biol.Chem. 2013, 288: 19614-19624.

47. Saini, A.; Chauhan, V.S., Delineation of the core aggregation sequences of TDP-43 Cterminal fragment. Chembiochem 2011, 12: 2495-2501. 
48. Furukawa, Y.; Kaneko, K.; Watanabe, S.; Yamanaka, K.; Nukina, N., A seeding reaction recapitulates intracellular formation of Sar insoluble transactivation response element (TAR) DNA-binding protein-43 inclusions. J. Biol. Chem 2011 286, 18664-18672.

49. Feiguin, F.; Godena, V.K.; Romano, G.; D'Ambrogio, A.; Klima, R.; Baralle, F.E., Depletion of TDP-43 affects Drosophila motoneurons terminal synapsis and locomotive behavior. FEBS Lett.2009, 583(10):1586-92.

50. Chang, C.K.; Chiang, M.H.; Toh, E.K.; Chang, C.F.; Huang, T.H., Molecular mechanism of oxidation-induced TDP-43 RRM1 aggregation and loss of function. FEBS Lett. 2013, 587: 575-582.

51. Cohen, T.J.; Hwang, A.W.; Restrepo, C.R.; Yuan, C.X.; Trojanowski, J.Q.; Lee, V.M., An acetylation switch controls TDP-43 function and aggregation propensity. Nat Commun. 2015, 5; 6:5845.

52. Yang, M.; Dutta, C.; Tiwari, A., Disulfide-Bond Scrambling Promotes Amorphous Aggregates in Lysozyme and Bovine Serum Albumin. JPCB 2015,119: 3969-3981.

53. Marston, F.A.O.; Hartley, D.L.; Solubilization of protein aggregates. Methods Enzymol., 1990,182, 264-276.

54. Rabilloud, T., Solubilization of proteins for electrophoretic analyses. Electrophores is, $1996,17,813-829$.

55. Herbert, B., Advances in protein solubilization for two-dimensional electrophores is. Electrophoresis, 1999, 20, 660-663.

56. Fang, Y.S.; Tsai, K.J.; Chang, Y.J.; Kao, P.; Woods, R.; Kuo, P.H., et.al. Full length TDP-43 forms toxic amyloid oligomers that are present in frontotemporal lobar dementia- TDP patients. Nature Communications 2013, 5:4824. 
57. Mompean, M., et.al. Structural evidence of amyloid fibril formation in the putative aggregation domain of TDP-43. The Journal of Physical Chemistry Letters 2015, 6: 2608-2615.

58. Sodhai, A., et.al. Aberrant assembly of RNA recognition motif 1 links to pathogenic conversion of TAR DNA- binding protein of $43 \mathrm{kDa}$ (TDP-43). J. Biol.Chem 2013, 288: 14886-14905.

59. Chang, C.K.; Chiang, M.H.; Toh, E.K.; Chang, C.F.; Huang, T.H., Molecular mechanism of oxidation-induced TDP-43 RRM1 aggregation and loss of function. FEBS Lett. 2013, 587: 575-582.

60. Mossuto, M.F.; Disulfide bonding in Neurodegenerative Misfolding Diseases. International journal of Cell Biology 2013, 10.1155 .

61. Toichi, k.; Yamanaka, K.; Furukawa, Y., Disulfide scrambling describes the oligo mer formation of superoxide dismutase (SOD1) proteins in the familial form of amyotrophic lateral sclerosis. J. Biol.Chem 2013, 288(7): 4970-80.

62. Zhang, T.; Mullane, P.C.; Periz, G.; Wang, J., TDP-43 neurotoxicity and protein aggregation modulated by heat shock factor and insulin/ IGF-1 signaling. Human Molecular Genetics 2011, 10: 1-14.

63. Wu, J.; Watson, T., A novel methodology for assignment of disulfide bond pairings in proteins. Protein Science 1996, 6:391-398. 
Chapter 4 Acetylation at lysine residues modulates Aß42 aggregates structure and cytotoxicity $^{\dagger}$

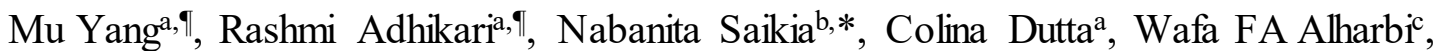
Zhiying Shanc, Ravindra Pandey ${ }^{\mathrm{b}}$, and Ashutosh Tiwaria,*

a Department of Chemistry, Michigan Technological University, Houghton, Michigan 49931, United States

${ }^{\mathrm{b}}$ Department of Physics, Michigan Technological University, Houghton, Michigan 49931, United States

c Department of Kinesiology and Integrative Physiology, Michigan Technological University, Houghton, Michigan 49931, United States

『 Both authors contributed equally to this work.

This chapter contains materials from a manuscript submitted to Scientific Reports for publication. 


\subsection{Introduction}

Protein misfolding and aggregation leads to several neurodegenerative diseases among which Alzheimer's disease (AD) is the sixth leading cause of death in U.S. with estimate of 5.8 million Americans living with the disease in 2019. Altheimer's is a progressive neurodegenerative disease leading to behavioral and cognitive disorder in the elderly ${ }^{1}$, and is characterized by the presence of senile plaques and neurofibrillary tangles. Senile plaque constitutes mainly of beta amyloid peptide and neurofibrillary tangles is composed of microtubule associated protein known as Tau ${ }^{2}$. The peptide of Amyloid $\beta$ vary in length from 39-43 amino acids. In normal individual, $A \beta 40$ is the major form of $A \beta$ produced and only 5-15\% of total $A \beta$ is comprised of $A \beta 42$. Though $A \beta 40$ is present in higher concentration in cerebrospinal fluid (CSF) compared to $\mathrm{A} \beta 42$, the amyloid plaques from $\mathrm{A} \beta 42$ constitutes the major component in $\mathrm{AD}$ brains.

The extracellular amyloid plaques recognized as one of the major hallmark of the disease, have been reported for variability of toxicity for different structures of $A \beta$ aggregates ${ }^{3-8}$. However, the relationship between different aggregated structures of $\mathrm{A} \beta$ peptides and its associated toxicity is not well understood. This is further underscored by the failure of $\mathrm{AD}$ treatment based on $A \beta$ aggregates as these treatments targeted mature amylo id fibril forms but failed to target highly transient and dynamic $A \beta$ species that are naturally formed in the brain $5,9,10$.

Post-translational modification is well known for its role in protein folding and aggregation. Likewise, while considering the in vivo "pool" of $\mathrm{A} \beta$, post-translation modified forms of $A \beta$ are present besides $A \beta$ peptides of different lengths ${ }^{7}$. Different post- 
translational modifications (PTMs) such as phosphorylation 11-13, truncation 14-16 isomerization ${ }^{17,18}$, and pyroglutamate formation ${ }^{16,19}$, were found to play role in $\mathrm{A} \beta$ peptide structure and aggregation propensity. In a recent study, protein PTM level were found to increase roughly two-fold in $\mathrm{AD}$ samples compared to normal controls, especially in $\mathrm{A} \beta$ and tau enriched area $^{21}$. Among the various post-translational modifications in proteins, acetylation is one of the major PTM that plays a vital role in health and diseases. Some major role in health includes DNA repair, protein structure, signaling, and protein selfassembly and is also involved in diseases like cancer and neurodegenerative diseases ${ }^{21-28}$. Interestingly, among all the identified PTM types in AD brains, acetylation only affects $10 \%$ of the total modified peptides but showed the highest increase level in A $\beta$ - and Taucontaining aggregates ${ }^{20}$. Acetylation of Tau has already been identified and is proposed for new therapeutic targets for $\mathrm{AD} \cdot{ }^{29-31}$ However, acetylation on $\mathrm{A} \beta$ peptide has not been investigated in detail. The A $\beta$ peptide has two potential acetylation sites, lysine 16 (K16) and lysine 28 (K28) (Figure 1A); the effect of K28 acetylation has been investigated, though $\mathrm{K} 16$ residue as an acetylation site was often ignored. Lysine 28 was recognized as a key player during $A \beta$ fibril formation as it forms a salt bridge with $A 42$ in $A \beta 42$ fibrils ${ }^{32}$, or with $\mathrm{D} 23$ in $\mathrm{A} \beta 40$ fibrils ${ }^{33}$, to stabilize the $\beta$-sheet structure.

We notice that a systematic study investigating the impact of acetylation of potential acetylation sites of $A \beta$ on protein aggregation and the effect of aggregates on biological health has not yet been reported. This is important because many common drugs and metabolites were recently found to be able to facilitate protein acetylation ${ }^{21,34}$. In this study, synthesized A $\beta 42$ peptides were modified with single acetylation on K16 (K16Ac), K28 (K28Ac), or were double acetylated on both Lysine residues (KKAc) (Figure $1 \mathrm{~B}-\mathrm{E})$. The 111 
fibril formation was simulated via assembly of 9 strands of $A \beta 42$ peptides. The effect of WT acetylated and 1:1 mixture peptides on cellular heath was measured using cell viability, cytotoxicity and free radical formation assays. Surprisingly, we found that side-chain acetylation of $\mathrm{K} 16$ can profoundly impair the fibrillization property of $\mathrm{A} \beta 42$ peptide and significantly affects the toxicity, which could account for the modulation of $\mathrm{A} \beta 42$ pathogenicity.

\section{A $A \beta_{42}$ : DAEFR HDSGY EVHHQ KLVFF AEDVG SNKGA IIGLM VGGVV IA}
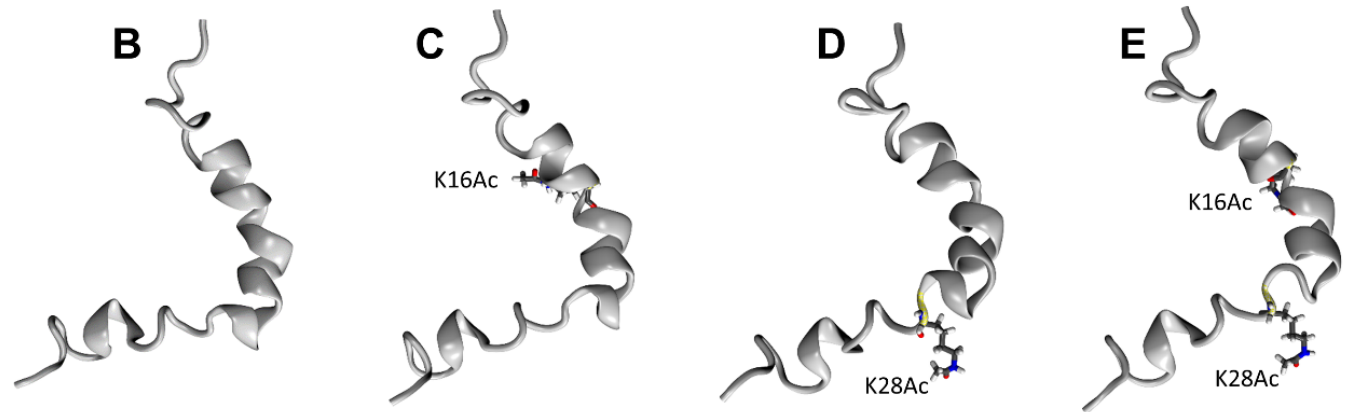

Figure 1. (A) Sequence of $\mathrm{A} \beta 42$ peptide (PDB entry: $1 \mathrm{Z} 0 \mathrm{Q}{ }^{33}$ ). Atomic structures of (B) WT, (C) K16Ac, (D) K28Ac, and (E) KKAc monomer. The two acetylated positions, K16 and $\mathrm{K} 28$, are highlighted in ball and stick.

\subsection{Methods}

Preparation of amyloid $\beta$ peptide samples - Peptides: Synthetic wild-type (WT), K16Ac, K28Ac and KKAc were purchased from Biomatik (Canada). All the peptides were confirmed for their identity and purity ( $>95 \%)$ by mass spectrometry and RP-HPLC. The peptides were dissolved as per the methods reported by Roychaudhuri et. al. ${ }^{36}$ In brief, the lyophilized peptide was first dissolved in $10 \%(\mathrm{v} / \mathrm{v})$ of $60 \mathrm{mM} \mathrm{NaOH}$, followed by $45 \%$ 
(v/v) MilliQ water. The $\mathrm{pH}$ was adjusted by adding $45 \%(\mathrm{v} / \mathrm{v})$ of $10 \mathrm{mM}$ sodium phosphate buffer $\left(\mathrm{pH}\right.$ 7.4). Then the centrifugation was carried out at $16,000 \times \mathrm{g}$ at $4{ }^{\circ} \mathrm{C}$ for 10 minutes. The supernatant fluid thus obtained was filtered through $0.22 \mu \mathrm{m}$ membrane. Concentration of peptide stock was determined using $\varepsilon 214=75,887 \mathrm{M}^{-1} \mathrm{~cm}^{-1}$ and BCA Assay. For experiments, $50 \mu \mathrm{M}$ peptide was mixed with $50 \mathrm{mM}$ phosphate buffer, 300 $\mathrm{mM} \mathrm{NaCl}, \mathrm{pH} 7.4$ and were incubated at $37^{\circ} \mathrm{C}$ with agitation for indicated time. All buffers, salts and MilliQ water were filtered through $0.22 \mu \mathrm{m}$ membrane. The peptide stocks were stored at $-80^{\circ} \mathrm{C}$.

Fluorescence measurements - All fluorescence measurements were carried out at the final concentration of $10 \mu \mathrm{M}$ in $10 \mathrm{mM}$ phosphate buffer, $\mathrm{pH}$ 7.4. Stocks of fluorescent probes 8anilino-1-naphthalene sulfonic acid (ANS), and 4,4'-dianilino-1,1'-binaphthyl-5,5'disulfonic acid (bis-ANS) were prepared in ethanol and then added to samples to final concentrations of $10 \mu \mathrm{M}$ (ANS), and $1 \mu \mathrm{M}$ (bis-ANS). Emission spectra were collected using a Horiba Jobin Yvon spectrofluorometer (Fluoromax-4) at room temperature. For samples with ANS and bis-ANS, spectra were acquired from $400-700 \mathrm{~nm}$, with excitation at $380 \mathrm{~nm}$ and $360 \mathrm{~nm}$, respectively.

Field emission scanning electron microscopy (FESEM) - Fibrils and aggregates of peptides were analyzed using a cold field emission high-resolution scanning electron microscope, Hitachi S-4700 FESEM. After incubation, fibrils or aggregates were pelleted by centrifuging at $9,000 \times \mathrm{g}$ for $45 \mathrm{~min}$ at $25^{\circ} \mathrm{C}$. To wash off salts, the pellets were suspended in MilliQ water $(0.22 \mu \mathrm{m}$ membrane filtered $)$ and centrifuged at $9,000 \times \mathrm{g}$ for $45 \mathrm{~min}$ at 25 ${ }^{\circ} \mathrm{C}$. Washed fibrils or aggregates were applied on SEM stubs and air dried at room 
temperature. The SEM samples were then coated with $10 \mathrm{~nm}$ platinum. For FESEM imaging, $10 \mathrm{kV}$ of acceleration voltage and $5 \mu \mathrm{A}$ of emission current were used.

Cell viability (MTS) assay - SH-SY5Y human neuroblastoma cells (both from ATCC) were cultured in Medium 199 and DMEM/F-12 medium, respectively, with 10\% FBS and 100 $\mathrm{U} / \mathrm{ml}$ penicillin-streptomycin at $37{ }^{\circ} \mathrm{C}$ in $5 \% \mathrm{CO}_{2}$ humidified environment and used within the first 10 passages. Cells were plated at $2 \times 10^{4}$ cells/well (SH-SY5Y) on 96-well plates and allowed to grow overnight. The next day, cells were washed with $1 \mathrm{X}$ PBS buffer $(\mathrm{pH}$ 7.4) twice. Then $100 \mu l$ fresh media containing peptide samples were added. Six replicates were prepared per sample. Media without any peptide were used as controls. After $48 \mathrm{~h}$ incubation, $20 \mu 1$ of CellTiter 96® AQueous One Solution Cell Proliferation (MTS) Assay kit (Promega) were added and incubated for $4 \mathrm{~h}$. Absorption at $490 \mathrm{~nm}$ were collected using an ELISA plate reader (BioTek Instruments, Inc.). Blanks containing media and peptide samples but no cells were similarly prepared and used for background subtraction.

Cytotoxicity (LDH) assay - SH-SY5Y human neuroblastoma cells (from ATCC) were cultured as described above and plated at $2 \times 10^{4}$ cells/well on 96-well plates and allowed to grow overnight. The next day, cells were washed with $1 \mathrm{X}$ PBS buffer $(\mathrm{pH}$ 7.4) twice. Then $100 \mu \mathrm{l}$ of fresh media containing peptide samples were added. Six replicates were prepared per sample. After $48 \mathrm{~h}$ incubation, $10 \mu 1$ of lysis solution (CytoTox 96® NonRadioactive Cytotoxicity Assay (LDH) Assay kit, Promega) was added to maximum LDH release control wells and incubated for 45 minutes at $37^{\circ} \mathrm{C}$ in $5 \% \mathrm{CO}_{2} .50 \mu$ of supernatant from all the wells were transferred into a new plate and to each of those wells $50 \mu \mathrm{l}$ of LDH reagent was added. The plate was further incubated in dark for 30 minutes. After 
incubation, $50 \mu$ l of stop solution was added to each well. Absorption at $490 \mathrm{~nm}$ were collected using a plate reader (BioTek Instruments, Inc.). Appropriate blanks containing media and peptide samples (without cells) were similarly prepared and used for background subtraction. Max LDH Control was represented as 100\% cytotoxic and all samples were normalized to Max LDH Control ${ }^{37}$. Primary hippocampal neuronal cells were prepared as described in the immunostaining protocol (below). The LDH assay was carried out for neuronal cells as described for SH-SY5Y cells above with the following variations. Neuronal cells were plated at $1 \times 10^{4}$ cells/well in 96-well plate. And the cells were incubated with $2 \mu \mathrm{M}$ of peptides in $100 \mu \mathrm{L}$ of media for $24 \mathrm{~h}$.

2',7'-dichlorofluorescin diacetate (DCFH-DA) fluorescence assay - SH-SY5Y human neuroblastoma cells (from ATCC) were cultured and were plated at $2 \times 10^{4}$ cells $/$ well on 96-well plates and allowed to grow overnight. The next day, cells were washed with $1 \mathrm{X}$ PBS buffer (pH 7.4) twice. Then, cells were incubated with $20 \mu \mathrm{M}$ DCFH-DA for 45 minutes at $37{ }^{\circ} \mathrm{C}$ in dark. After incubation, the cells were washed twice with $1 \mathrm{X}$ PBS (pH 7.4) and then $100 \mu \mathrm{l}$ of fresh media containing peptide samples were added to DCFH-DA (Sigma) treated cells in five replicates and incubated for $24 \mathrm{~h}$ at $37{ }^{\circ} \mathrm{C}$ in $5 \% \mathrm{CO}_{2}$ humidified environment. Cells without any treatment and blanks were also incubated under identical condition. For positive control, cells after DCFH-DA treatment were incubated with $50 \mu \mathrm{M}$ of t-butyl-hydroperoxide (tBHP) for $4 \mathrm{~h}$ at $37^{\circ} \mathrm{C}$. Finally, the fluorescence intensity was measured using fluorescence plater reader (Fluoroskan Ascent, Thermo Scientific) at excitation wavelength of $485 \mathrm{~nm}$ and emission wavelength of $538 \mathrm{~nm}$. All data were normalized to tBHP wherein tBHP treated cells were represented as $100 \%$ free radical generating ${ }^{38}$. DCFH-DA assay for primary hippocampal neuronal cells was same 115 
as that detailed for SH-SY5Y cells above with the following variations. Neuronal cells were plated at $1 \times 10^{4}$ cells/well in 96-well plate. And the cells were incubated with $2 \mu \mathrm{M}$ of peptides in $100 \mu \mathrm{L}$ of media for $24 \mathrm{~h}$.

Immunostaining of primary hippocampal neuronal cells - Sprague Dawley rats were purchased from Charles River Laboratories (Wilmington, MA, USA) and used in our breeding colony to generate1-day-old pups which were euthanized via overdose of pentobarbital. Hippocampus and cortex from 1-day old rats were dissected, combined and neuron cells dissociated as detailed previously ${ }^{39}$. Cells were plated in poly-L-Lysine coated 24 well plates at the seeding density of $5 \times 10^{4}$ cells/well and incubated at neurobasal medium containing B27 supplement (Thermo Fisher Scientific) in $37^{\circ} \mathrm{C}$ incubator. Culture medium was changed every 3 days and the cells were allowed to grow for $10 \sim 14$ days prior to their use in experiment. During the experiment, $500 \mu \mathrm{l}$ of fresh media containing $2 \mu \mathrm{M}$ of $7 \mathrm{~d}$ A $\beta 42$ peptide aggregates (WT and acetylated) were added to neuronal cells and incubated for $24 \mathrm{~h}$ at $37^{\circ} \mathrm{C}$ incubator. Control cells did not have any peptides added to them but were incubated similarly. After $24 \mathrm{~h}$, the cells were washed twice with PBS. Then cells were fixed with 4\% paraformaldehyde for 10 minutes at room temperature. Cells were then washed with PBS three times for 5 minutes each. Blocking solution (5\% horse serum and $0.3 \%$ Triton $\mathrm{X}-100$ in PBS) was added to each well and incubated for 1 hour. After one hour, cells were incubated overnight at $4^{0} \mathrm{C}$ in a cocktail consisting of 1:200 dilution of mouse anti-tau antibody (Invitrogen, catalog\# AHB0042) and 1:100 dilution of rabbit anti- $\beta$-tubulin antibody (Cell Signaling Technology, Catalog\# 15115S). Afterwards, cells were washed in PBS thrice for 5 minutes and incubated overnight at $4^{0} \mathrm{C}$ in a mixture of secondary antibodies (Alexa Fluor 594 116 
donkey anti-mouse IgG and Alexa Fluor 488 donkey anti-rabbit IgG, both in 1:300 dilution). The sections were mounted in Vectashield (Vector Labs, Burlingame, CA) and images were taken with a Leica fluorescence microscope (LEICA DM IL LED) at 20X and were analyzed using Image $J$ software ${ }^{40,41}$

Statistical analysis - Statistical analysis was performed using one-way ANOVA and posthoc t-test. The $p$ values $<0.05$ were considered as significant and are represented *, $\mathrm{p}<0.005$ represented as $* *$ and $\mathrm{p}<0.0005$ represented as $* * *$.

Computational methods - To construct the acetylated (K16Ac, K28Ac and KKAc) peptides, we started with the WT A $\beta 42$ monomer as shown in Figure 1B (PDB entry: 1Z0Q). The $\mathrm{NH}_{3}{ }^{+}$-group of Lysine 16 and Lysine 28 residues of $\mathrm{A} \beta 42$ monomer was modified by substituting with an acetyl-group in the side-chain. Likewise, initial configurations of 9-strand aggregates of A $\beta 42$ fibrils (WT and acetylated) were constructed by aligning the individual $\mathrm{A} \beta 42$ monomers parallel to one other, avoiding any close contacts or atomic clashes during the assembly. The A $\beta 42$ monomers and fibril structures were initially placed in an periodic orthorhombic box and solvated with water molecules defined using the TIP3P model ${ }^{38}$. The $\mathrm{H}$-atoms of water molecules were constrained using the SETTLE algorithm ${ }^{39}$ and counter ions were added to maintain overall charge neutrality of the system. The details of system size are provided in Supplementary Information, Table 2.

Atomistic MD simulations of WT and acetylated $\mathrm{A} \beta 42$ peptides were performed in the isothermal-isobaric (NPT) ensemble using NAMD program and CHARMM27 force field. The simulation temperature $(310 \mathrm{~K})$ and pressure $(101.3 \mathrm{kPa})$ was maintained using the 
Langevin dynamics and Langevin piston Nose-Hoover method ${ }^{40}$. The long-range electrostatic interactions were calculated using the particle mesh Ewald method with a cutoff of $12.0 \AA^{41}$. For each system, we performed energy minimization for 2000 steps at a time step of $1.0 \mathrm{fs}$ using the conjugate gradient method, followed by $100 \mathrm{~ns}$ of production run at a time-step of $1 \mathrm{fs}$. We performed a total of $800 \mathrm{~ns}$ runs for all systems and each simulation run was repeated for reproducibility of the results. Simulation snapshots corresponding to structural changes, and convergence of MD simulation trajectory was analyzed using the backbone root-mean-square deviation (RMSD) for the peptides using VMD 1.9.2. program ${ }^{42}$. The surface hydrophobic patches for 9 strands of WT and acetylated (K16Ac, K28Ac, and KKAc) A $\beta 42$ peptides in aqueous solution were analyzed using the SPDB software.

\subsection{Results}

\section{Physicochemical characterization of $A \beta$ peptides aggregation}

The fibrillization/aggregation properties of WT A $\beta$, acetylated peptides and 1:1 mixture ratios was already monitored by ThT fluorescence and reported in Dissertation work of Yang. M(2016). Based on the findings WT, K28Ac and WT:K28Ac followed the sigmo idal fluorescence trend whereas K16Ac and K28Ac showed non-sigmoidal Tht fluorescence trend. Further to verify the aggregates, FESEM was carried out for $7 \mathrm{~d}$ incubated samples . In line with the previously reported data for Tht and FESEM, the new batch of peptides displayed similar morphology for 7d incubated samples (Figure 2). The WT, K28Ac and WT:KKAc was able to form fibril like structures upon incubation for 7 days while K16Ac, KKAc and 1:1 mixture of both with WT remained amorphous even after incubation for 7 
days (Figure 2). All peptides of $A \beta$ remained amorphous in nature upto $72 \mathrm{~h}$ incubation (Supplementary information 1).

Next, we determined the structure flexibility of WT, acetylated and 1:1 mixture of A $\beta$ peptides by using the structure sensitive probe bis-ANS. We found that K16Ac and KKAc have flexible structure compared to other peptides. Among all peptides K16Ac showed the most structure flexibility while KKAc aggregates are more denser compared to K16Ac (Figure 3). Furthermore, the mixtures of single and double acetylated peptides with WT A $\beta 42$ peptide (1:1 ratio) formed more rigid amorphous aggregates with fluorescence signal comparable to WT A $\beta 42$ aggregates than those formed by pure K16Ac peptides (Figure 3).

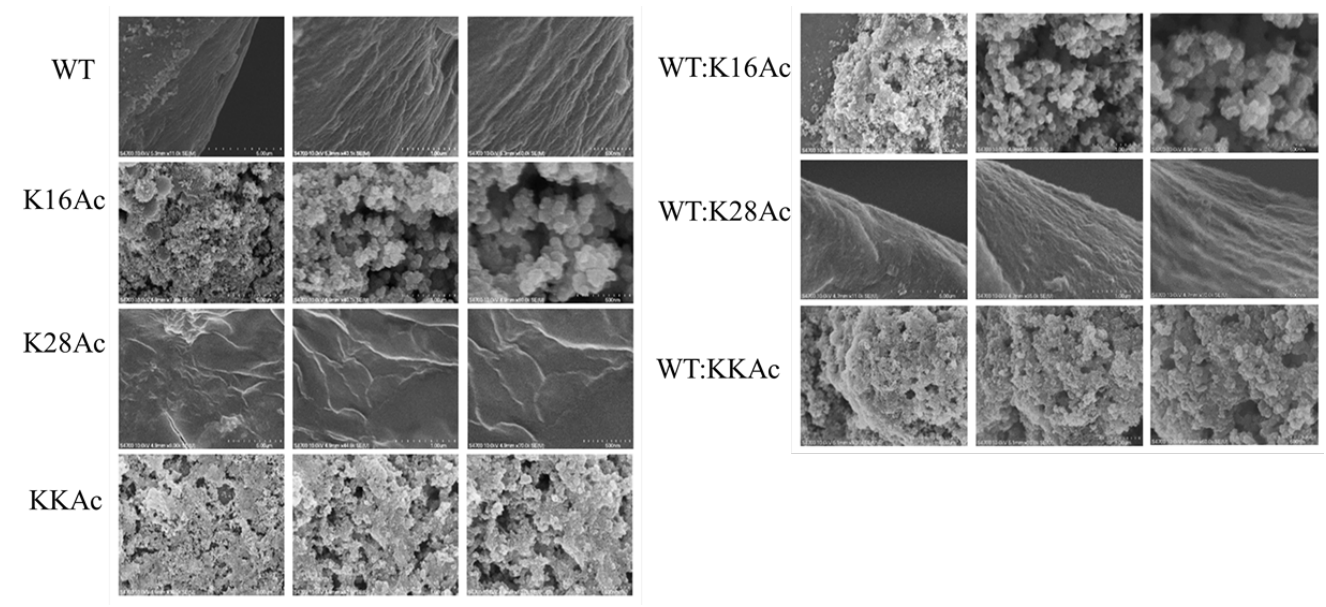

Figure 2. The morphology of the 7d incubated WT, acetylated and 1:1 mixtures confirmed by SEM. WT, K28Ac and WT:K28Ac forms fibrils while K16Ac, KKAc, WT:K16Ac and WT:KKAc remains amorphous in nature under $7 \mathrm{~d}$ incubation. Scale bar $=5 \mu \mathrm{m}, 1 \mu \mathrm{m}$ and $500 \mathrm{~nm}$ from left to right, respectively. 


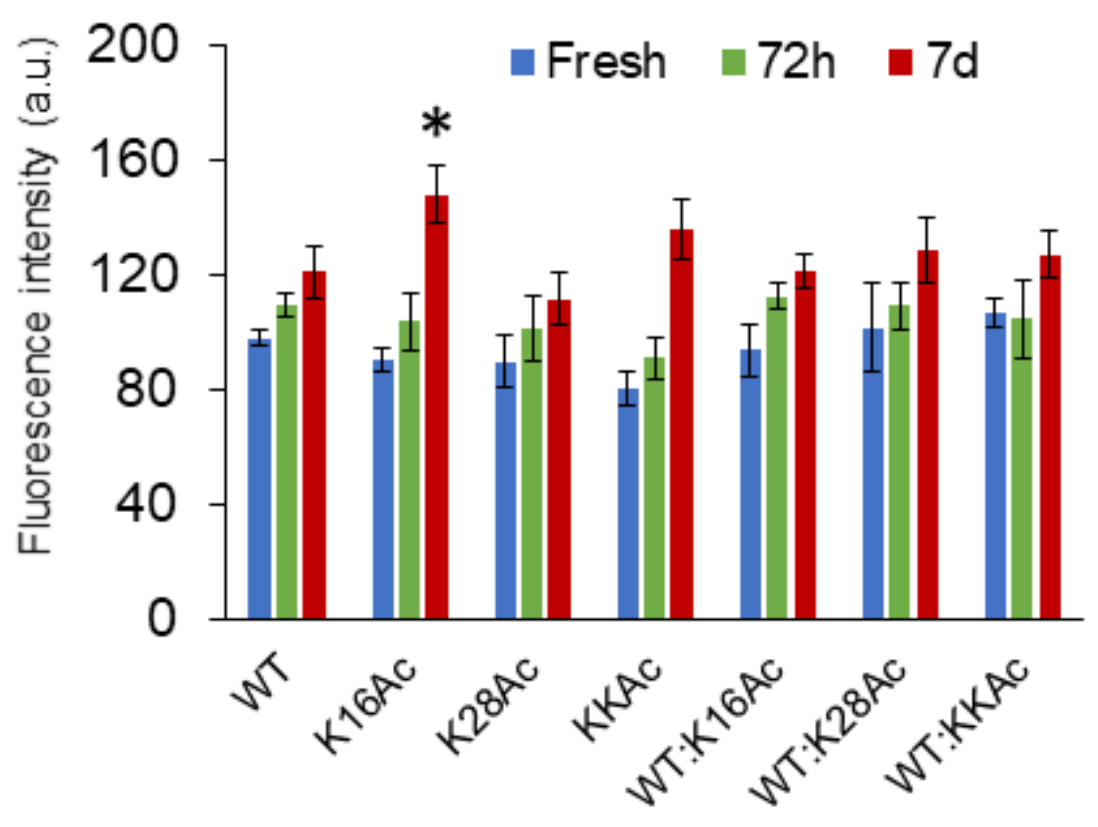

Figure 3: Structure flexibility of the fresh $A \beta 42$ peptides and their aggregates were measured by Bis-ANS fluorescence by incubating $10 \mu \mathrm{M}$ of peptide samples with $1 \mu \mathrm{M}$ of Bis-ANS and measuring peak intensities at $484 \mathrm{~nm}$. Mixture represents the 1:1 ratio of the acetylated peptides with WT A $\beta 42$ peptides. Error bars $= \pm$ S.D, $(n=3)(*$ represents significant differences $(p<0.05)$.

Molecular aggregation dynamics and surface properties of $A \beta$ peptides

To gain atomistic insights into the conformation changes, aggregation dynamics, and map the folding pathways of A $\beta 42$ peptides ${ }^{43-49}$, we performed molecular dynamics (MD) simulations of WT and acetylated A $\beta 42$ monomers along with 9 strand aggregates of all four peptides (WT, K16Ac, K28Ac, and KKAc) in aqueous solution using NAMD program ${ }^{50}$ and CHARMM27 force field ${ }^{51}$. Note that aggregation of 9 strands of WT and acetylated peptides were considered as replica models for simulating the formation of fibril- like 
structures. Supplementary Figure 2 A-D displays the structural changes in WT and acetylated A $\beta 42$ monomer in aqueous solution at time $=0$ and100 ns. WT A $\beta 42$ is mostly $\alpha$-helical at residues $11-23$ and 29-37, which agrees well with previously reported results based on the discrete MD simulation method ${ }^{52}$. In our case of atomistic MD study, the flexible regions are the first 10 amino acids of $\mathrm{N}$-terminus, residues $38-42$ of $\mathrm{C}$-terminus that adopt a random coil structure, and the $\beta$-turn region between residues 24-28 (Supplementary Figure 3A). Snapshots of WT and acetylated peptide aggregates at time = 0 and 100 ns elucidate that the nature of fibrillization is distinctive of the modifications: K16Ac and KKAc assemble into an amorphous structure while K28Ac retains a fibril- like structure similar to WT peptides (Figure 2). MD simulation snapshots (Supplementary Figure 6) of K16Ac at $100 \mathrm{~ns}$ affirm the formation of amorphous aggregate as seen in SEM micrographs (Figure 2). A comparison of the orientation of K16 and K28 residues in acetylated aggregates suggests that in the former structure, K16Ac residues are somewhat embedded within the aggregate, stabilized by inter-chain hydrophobic interactions while in the latter, K28Ac residues project outwards with reduced inter-chain interactions (Supplementary Figure 4). Both MD simulation and experimental results thus find that K16Ac presents a flexible structure with loss of $\alpha$-helicity and acetylation significantly impacts aggregation dynamics and impedes fibril formation. Root mean square deviation (RMSD) analysis was performed for the simulations of each system (Supplementary Figure 9). RMSD of WT and acetylated peptides increases in the first 20 ns and then reaches a plateau, suggesting an overall stability of the system. A slightly higher RMSD of K16Ac correlate to the molecular flexibility and observed conformational transition to an amorphous structure. 


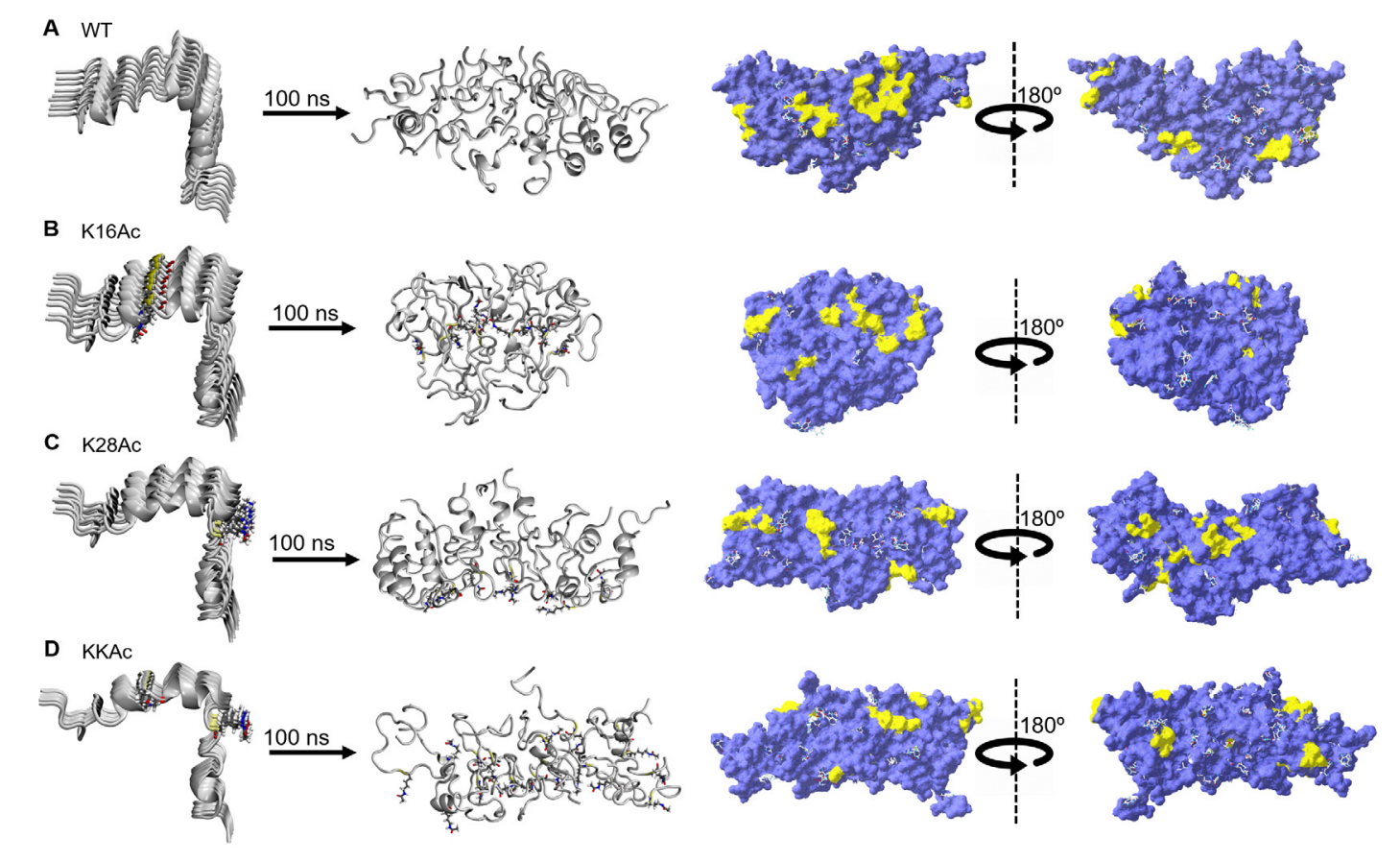

Figure 4. Simulation snapshots depicting the structural changes in aggregation of 9 strands of WT and acetylated (K16Ac, K28Ac and KKAc) A $\beta 42$ peptides in aqueous solution. For each structure, the predicted hydrophobic patches (yellow) were mapped on the molecular surface (violet) using SPDB software. (A) WT, (B) K16Ac, (C) K28 Ac and (D) KKAc aggregates. The waters molecules are not shown for clarity. (The computational work was conducted by Professor Ravindra Pandey and Dr. Nabanita Saikia . The figure 3 is added with their permission). 
Hydrophobic patches on a protein surface identify accessible regions of neighboring apolar atoms that may play a crucial role in protein folding, structural dynamics, and proteinligand interactions ${ }^{53}$. Surface hydrophobicity of WT and acetylated peptide aggregates were analyzed for $100 \mathrm{~ns}$ structures (Figure 4). Note that acetylation of the positively charged K16 and K28 lysine residues into hydrophobic moiety destabilizes fibril formation with an overall increase in the surface area (Supplementary Table 1). Interestingly, the surface area of KKAc is intermediate to K16Ac and K28Ac with relatively scattered hydrophobic patches on the molecular surface. K16Ac has the highest surface area and consequently exhibits a greater flexibility to adopt an amorphous structure. WT A $\beta 42$ peptides have one patch above $300 \AA^{2}$ and 9 patches between 100-300 $\AA^{2}$ (Supplementary Table 1). Acetylation leads to loss of the dominant patch above $300 \AA^{2}$ followed by decreases in size of the hydrophobic patches but increases the number of patches on the molecular surface. For acetylated peptides, the hydrophobic patches range between 100$300 \AA^{2}$. These findings are consistent with bis-ANS fluorescence data (Figure 3) that shows increased fluorescence for $7 \mathrm{~d}$ aggregates of K16Ac peptides compared to WT A $\beta 42$ peptides.

\section{Surface hydrophobicity of Aß peptides}

Surface hydrophobicity of the peptides and aggregates were also measured by ANS fluorescence (Figure 5). All three fresh acetylated peptides showed ANS fluorescence similar to WT A 342 peptides. Interestingly, after 3 days of incubation, ANS fluorescence of WT A $\beta 42$ peptides increased significantly compared to acetylated peptides and then 
decreased upon longer incubation of $7 \mathrm{~d}$ to levels comparable to fresh WT A $\beta 42$ peptides (Figure 5). Differences in hydrophobicity among the four peptides were amplified after 7 days of incubation, and was extremely significant for K16Ac peptides that almost doubled (Figure 5). Seven day incubated K28Ac A 42 peptides showed fluorescence signal that were significant and KKAc aggregates showed signal that were highly significant compared to WT peptides (Figure 5). In addition, for 7 day's samples incubated as mixture, the aggregates showed slightly less hydrophobicity compared to pure K16Ac and KKAc peptides but signals were significant compared to pure WT A $\beta 42$ aggregates (Figure 5).

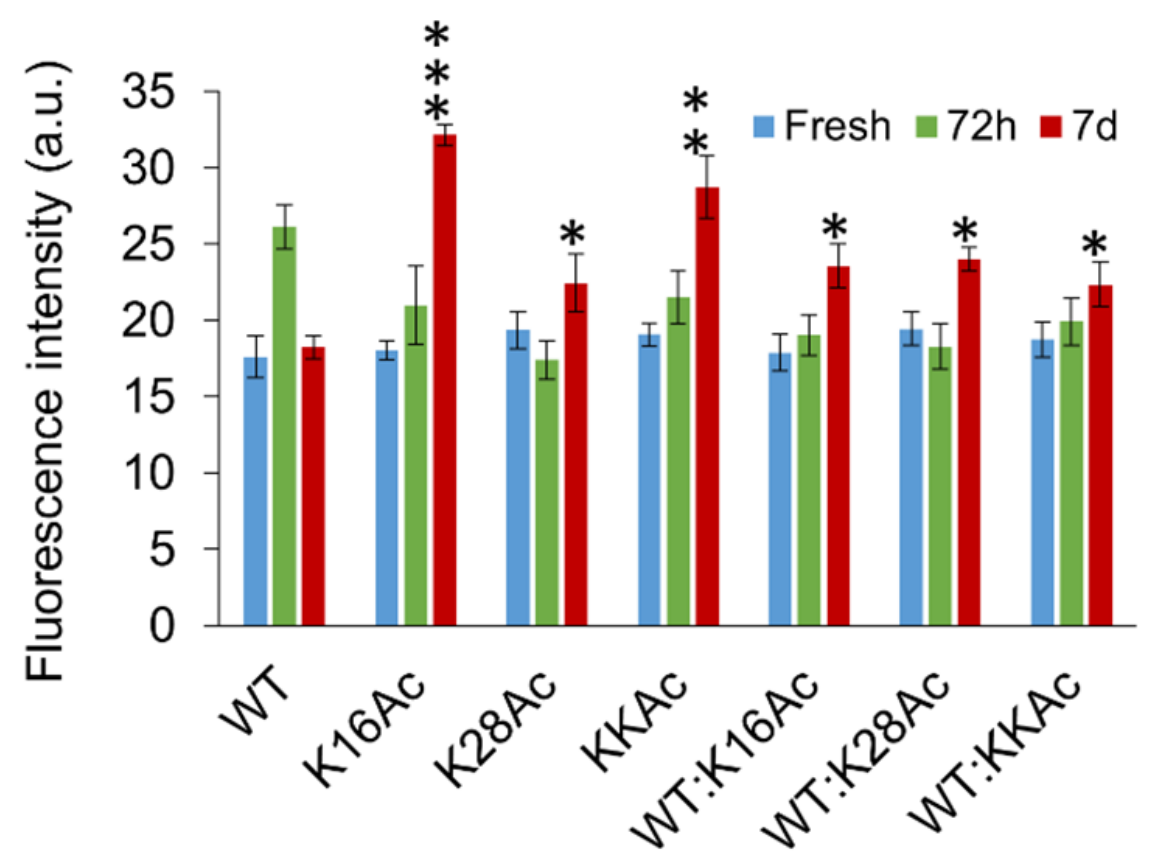

Figure 5. Hydrophobicity of fresh A $\beta 42$ peptides and their aggregates measured by ANS fluorescence by incubating $10 \mu \mathrm{M}$ of peptide samples with $10 \mu \mathrm{M}$ of ANS and measuring peak intensities at $485 \mathrm{~nm}$. Mixture represents the 1:1 ratio of the acetylated peptides with 
WT A $\beta 42$ peptides. Error bars $= \pm$ S.D. $(n=3) . *, * *$, and $* * *$ represents significant differences $(*=p<0.05),(* *=p<0.005)$, and $(* * *=p<0.0005)$.

\section{Cellular toxicity of A $\boldsymbol{\beta}$ peptides.}

In addition to measuring the morphology, surface hydrophobicity and structure flexibility of $\mathrm{A} \beta 42 \mathrm{WT}$, acetylated and 1:1 mixture, we investigated the effect of these aggregates on biological health of the cells. For this purpose, we tested the effect of two different concentration of peptide samples $(2$ and $5 \mu \mathrm{M})$ on SHSY-5Y neuroblastoma cells health (Supplementary Figure 12 and Figure 6) and $2 \mu \mathrm{M}$ A $\beta 42$ peptides on primary neuronal cells from rat (Figure 7). Based on the previous work reported by Yang, M et.al. (2016) on Tht fluorescence response of these peptides at different time points, we choose to carry out all cellular experiments at three different time points (Fresh, 72h and 7d). We monitored the effect of A 342 fibrils and aggregates on cell viability of SH-SY5Y neuroblastoma cells by MTS assay (Figure 6A and Supplementary Figure 11). The data shows that WT and single acetylated peptides slightly decrease SH-SY5Y cell viability but $7 \mathrm{~d}$ aggregates of KKAc peptides or 1:1 mixture of WT: K16Ac, and WT: K28Ac inhibit cell growth significantly (Figure 6A). And the 1:1 mixture of WT: KKAc peptides inhibit cell growth significantly more than KKAc peptides alone (Figure 6A).

Oxidative stress has been implicated in several neurodegenerative diseases including Alzheimer's disease ${ }^{54-60}$. Therefore, we wanted to measure effect of $5 \mu \mathrm{M}$ of A $\beta 42$ peptides (fresh as well as aggregates) on generation of reactive oxygen species (ROS) in cells. We used 2',7'-dichlorofluorescein diacetate (DCFH-DA) as a fluorescent probe to measure 
levels of ROS in cells (Figure 6B) ${ }^{36}$. Compared to untreated cells the cells treated with WT Aß42 peptides showed significant increase in ROS production in cells. The $7 \mathrm{~d}$ aggregated acetylated peptides K16Ac, K28Ac, and KKAc peptides showed much higher production of ROS in cells (Figure 6B). Interestingly, the 1:1 mixture of WT and all acetylated $\mathrm{A} \beta 42$ peptides also showed much higher production of ROS compared to the pure peptides aggregates with WT:KKAc peptide aggregates showing the highest ROS production (Figure 6B). To better understand the implication of $\mathrm{A} \beta 42$ peptides aggregates on ROS production and hence cell health, we also measured toxicity of these peptides by lactate dehydrogenase (LDH) cytotoxicity assay (Figure 6C) ${ }^{35}$. The aggregates from pure WT Aß42, K16Ac, and K28Ac peptides and equimolar mixture with WT (1:1) all show highly significant increase in toxicity for SH-SY5Y cells for $7 \mathrm{~d}$ aggregates consistent with increased ROS production (Figure 6B, C). Interestingly, among the group double acetylated peptides (KKAc) aggregates showed the highest toxicity (Figure 6C). Equimolar mixtures (1:1) of WT and KKAc $7 \mathrm{~d} A \beta 42$ peptides aggregates showed much higher increase in ROS production compared to pure KKAc (Figure 6B). However, the mixtures observed cytotoxicity was comparable to pure KKAc aggregates (Figure 6C). This maybe because both aggregates (pure KKAc and 1:1 mixture of WT:KKAc) are extremely toxic and values are close to Max LDH value which is the positive control (Figure 6C). 

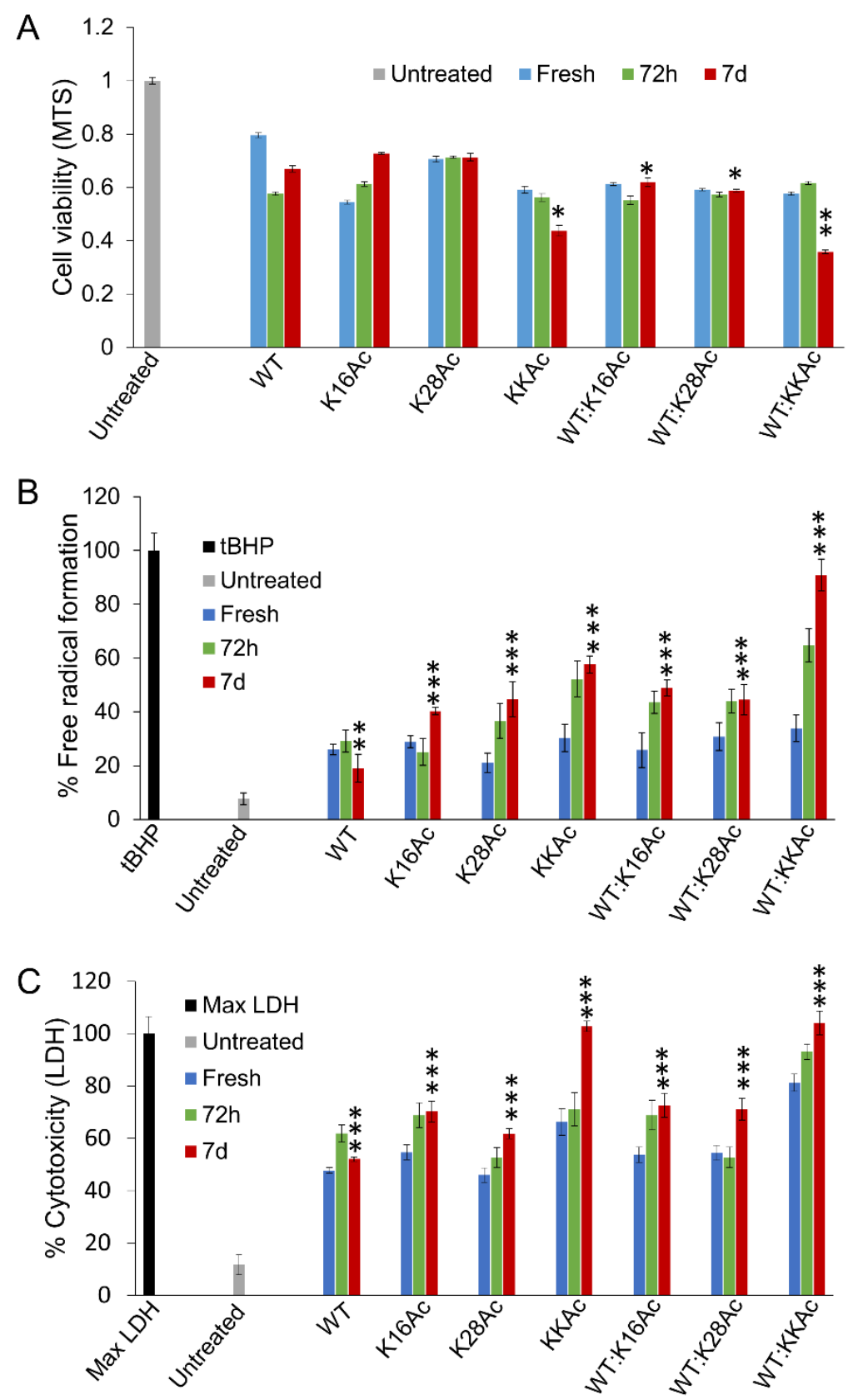

Figure 6. The effect of $5 \mu \mathrm{M}$ fresh $\mathrm{A} \beta 42$ peptides and their aggregates on SH-SY5Y neuroblastoma cells health were measured by (A) MTS assay, (B) DCFH-DA assay, and (C) LDH assay. The fresh peptides, $72 \mathrm{~h}$, or $7 \mathrm{~d}$ aggregates were added to the cells to a final 
concentration of $5 \mu \mathrm{M}$. Cells were incubated for $48 \mathrm{~h}$ in presence of peptides for MTS and LDH assay and for $24 \mathrm{~h}$ for DCFH-DA assay. For t-butyl-hydroperoxide (tBHP) control for DCFH-DA assay, cells were incubated with $50 \mu \mathrm{M}$ of tBHP for $4 \mathrm{~h}$ at $37^{\circ} \mathrm{C}$. The mixtures represent samples containing equimolar (1:1) WT and acetylated peptides. Error bars $= \pm$ S.D. (for $\mathrm{A} n=6$; for $\mathrm{B} n=5$; and for $\mathrm{C} n=5$ ). *, **, and $* * *$ represents significant differences $(*=p<0.05),(* *=p<0.005)$, and $(* * *=p<0.0005)$.

To further validate results on SH-SY5Y neuroblastoma cells, we carried out toxicity and ROS studies on primary neuronal cells from rat (Figure 7). Since $5 \mu \mathrm{M}$ of peptides was extremely toxic to SH-SY5Y neuroblastoma cells we used a lower concentration of peptides $(2 \mu \mathrm{M})$ to evaluate cytotoxicity and ROS production for primary neuronal cells. We incubated neuronal cells with $2 \mu \mathrm{M}$ of WT and acetylated A $\beta 42$ peptides for $24 \mathrm{~h}$ before the assay. Compared to the untreated neuronal cells, cells treated with $7 \mathrm{~d}$ aggregates of WT A 342 peptides and K28Ac peptides show a highly significant increase in ROS formation (Figure 7A). Cells treated with $7 \mathrm{~d}$ aggregates from K16Ac, KKAc, and equimolar mixtures (1:1) of WT and acetylated A $\beta 42$ peptides showed much higher increase in ROS production (Figure 7A). Consistent with increased ROS production, all neuronal cells treated with $2 \mu \mathrm{M}$ WT and acetylated A $\beta 42$ peptides and their (1:1) mixtures showed an extremely significant increase in cytotoxicity as measured by LDH assay (Figure 7B). The double acetylated A 42 peptides (KKAc) showed the highest increase in cytotoxicity as well as ROS production (Figure 7). 

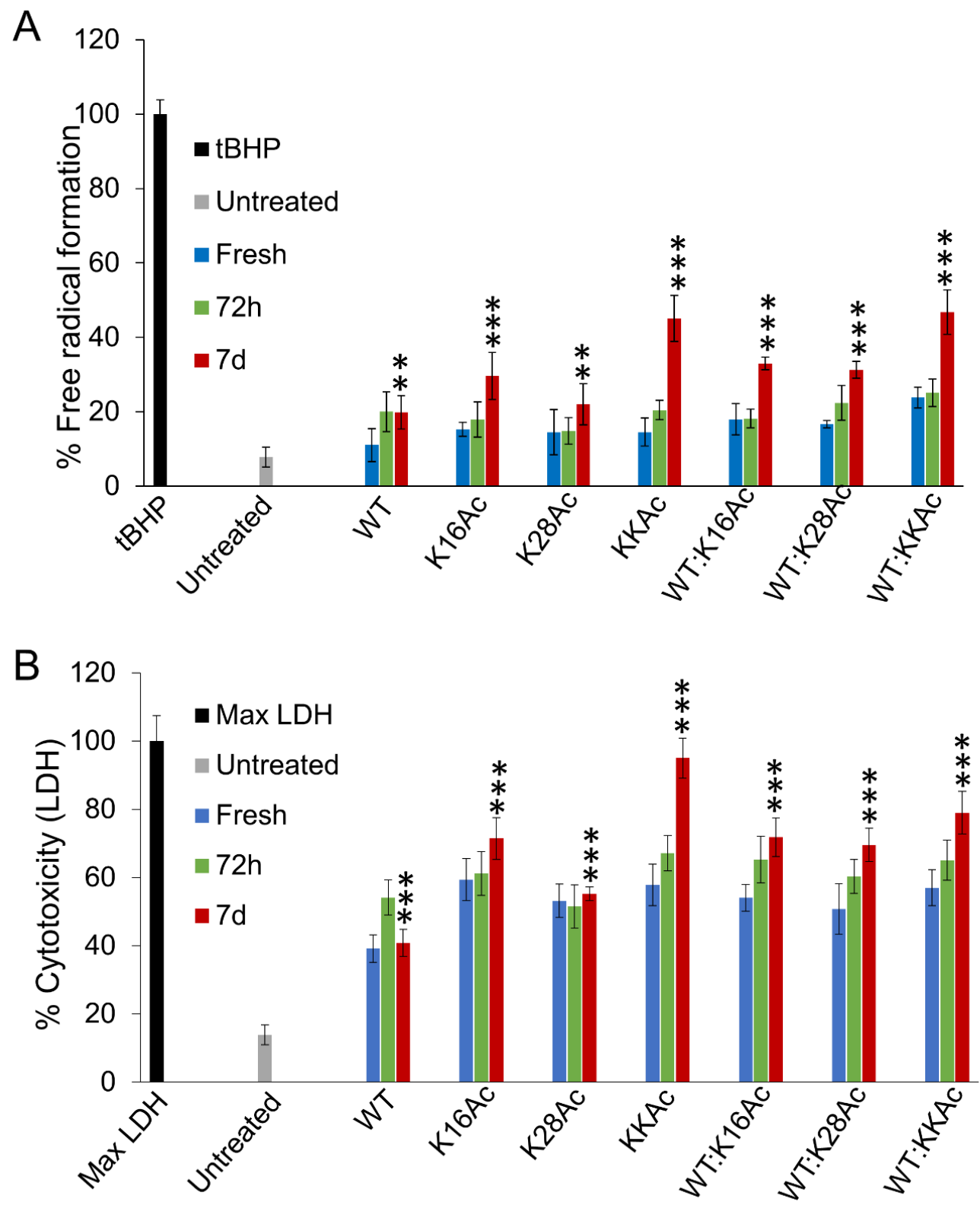

Figure 7. The effect of $2 \mu \mathrm{M}$ fresh $\mathrm{A} \beta 42$ peptides and their aggregates on primary neuronal cells health were measured by (A) DCFH-DA assay, and (B) LDH assay. The fresh peptides, $72 \mathrm{~h}$, or $7 \mathrm{~d}$ aggregates were added to the cells to a final concentration of $2 \mu \mathrm{M}$. 
Cells were incubated for $24 \mathrm{~h}$ in presence of peptides for both assays. For t-butylhydroperoxide (tBHP) control for DCFH-DA assay, cells were incubated with $50 \mu \mathrm{M}$ of tBHP for $4 \mathrm{~h}$ at $37^{\circ} \mathrm{C}$. The mixtures represent samples containing equimolar (1:1) WT and acetylated peptides. Error bars $= \pm$ S.D. (for $\mathrm{A} \mathrm{n}=5$; and for $\mathrm{B} \mathrm{n}=4$ ). ${ }^{*}, * *$, and ${ }^{* * *}$ represents significant differences $(*=p<0.05),(* *=p<0.005)$, and $(* * *=p<0.0005)$.

Immunostaining of primary neuronal cells treated with WT and acetylated AB42 peptides. In order to visualize the effect of WT and acetylated A $\beta 42$ peptides on primary neuronal cells, we carried out immunostaining of cells after treating them with $2 \mu \mathrm{M}$ of $7 \mathrm{~d} A \beta 42$ peptides aggregates for $24 \mathrm{~h}$ (Figure 8). We chose $2 \mu \mathrm{M}$ peptides concentration based on our ROS and LDH cytotoxicity assay for neuronal cells (Figure 7). We compared the morphology of neuronal cells treated with A 42 peptides for $24 \mathrm{~h}$ to control cells (untreated cells) that were similarly incubated by immunofluorescence microscopy (Figure 8). We used anti-tubulin antibody (green) to image tubulin protein and anti-Tau antibody (red) to image Tau protein in cells. The control cells (untreated primary neuronal cells) show a normal morphology with Tau signal that is diffused and evenly distributed in neurons (both soma and neurites) (Figure 8). The neuronal cells treated with $2 \mu \mathrm{M}$ of WT, K16Ac, and KKAc A $\beta 42$ peptides for $24 \mathrm{~h}$ showed a significantly higher immunofluorescence signal for Tau in soma of neurons compared to neurites (Figure 8, Supplementary Figure 15). Cell bodies of primary neurons showed the highest Tau signal for cells treated with KKAc peptides, high Tau signal for cells treated with WT and K16Ac peptides, and a much lower Tau signal for cells treated with K28Ac peptides (Figure 8). These findings are consistent with the ROS production and cytotoxicity data reported for these cells (Figure 8). 


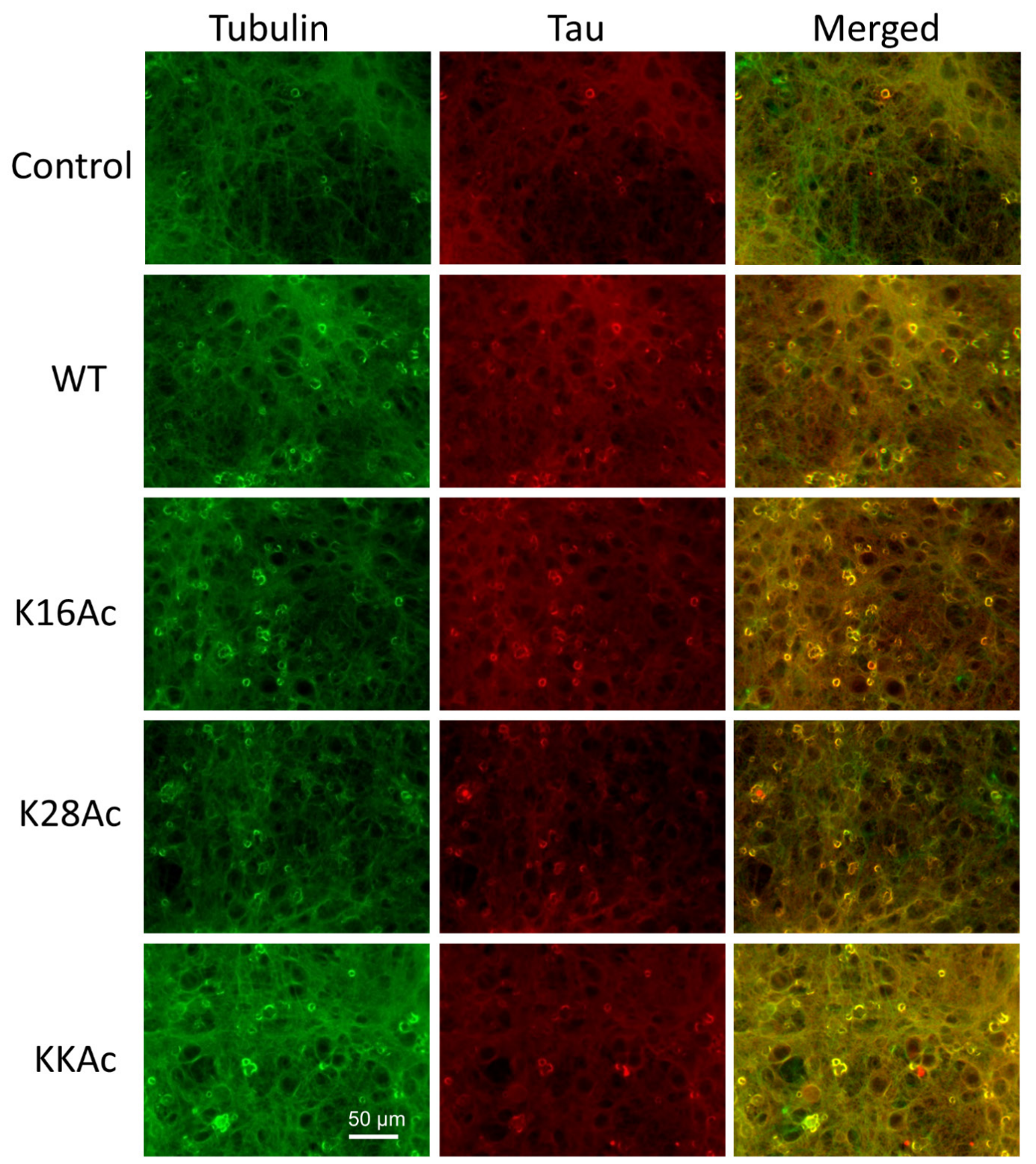

Figure 8. Immunostaining of primary neuronal cells. Primary neuronal cells were incubated with $2 \mu \mathrm{M}$ of $7 \mathrm{~d}$ incubated $\mathrm{A} \beta 42 \mathrm{WT}$ and acetylated peptides for $24 \mathrm{~h}$ at $37{ }^{0} \mathrm{C}$. Control indicates cells that are untreated and incubated under identical conditions with which $A \beta 42$ treated cells were compared. The cells were fixed and then stained for 
immunofluorescence with antibodies for tubulin (green) and Tau (red). The images were acquired using Leica DMIL LED at 20X magnification. Scale bar $=50 \mu \mathrm{m}$.

\subsection{Discussion}

Scientific literature suggests that formation of highly ordered amyloid fibril or less ordered oligomer structures could be an outcome of the competition between hydrogen bonding and hydrophobic interactions, as the higher ordered structures are usually stabilized by high density of direct inter-chain hydrogen bonds and steric zipper interactions 61,62. Furthermore, amyloid fibril dynamics could be affected by the chemical nature of the $\mathrm{A} \beta$ peptide through side-chain interactions and/or post-translation modifications ${ }^{63}$. In theory, introducing an acetyl moiety to a lysine residue removes a charge and hence can increase surface hydrophobicity of the peptide. Interestingly, in this study we showed that acetylation of lysine 16 and 28 on A $\beta 42$ does not affect protein aggregation the same way; K16 acetylation affects the aggregation morphology as well as kinetics very severely.

We found that the K16Ac peptide is much more hydrophobic with a higher structure flexibility than WT and other two acetylated peptides (Figures 3,5). The structural changes on $\mathrm{A} \beta 42$ monomer in aqueous solution (Supplementary Figure 2) agree well with a previously reported results based on the discrete MD simulation method ${ }^{52}$. Acetylation of A $\beta 42$ monomer at K16 leads to one extra $\beta$-turn between residues 14-17 (Supplementary Figure 3B) but acetylation at K28 retains a structure very similar to WT (Supplementary Figure $3 \mathrm{C}$ and $\mathrm{A}$ ). In addition, acetylation of $\mathrm{A} \beta 42$ at $\mathrm{K} 16$ and $\mathrm{K} 28$ residues on $\mathrm{KKAC}$ introduces a pleated region between residues 31-37 with loss in $\alpha$-helix (Supplementary 
Figure 3D). In agreement with our MD results, a rapid aggregation kinetics with no lag time was reported in a simulation study of $A \beta 16-22$ fragment, where the $A \beta 16-22$ oligomers were disordered molten globular structures due to strong hydrophobic interactions ${ }^{61}$, since K16 located in the central hydrophobic core, L17-F20, and the 16-20 residues (KLVFF) acts as a docking motif of $A \beta$ fibrillation ${ }^{33}$. This fact is also supported by our MD simulations of 9 strands K16Ac aggregates, where the K16Ac residues are embedded inside the aggregate structure, stabilized by the inter-chain hydrophobic intercalations (Supplementary Figure 4). A $\beta$ fibril grows in two distinct steps: a rapid and reversible "dock" phase, followed by a slow but irreversible "lock" phase ${ }^{64}$. In case of K16Ac peptide aggregation as observed in the current study, it is possible that the interchain hydrophobic interactions of the 16-20 region are enhanced after removal of the positive charge on K16 (Figure 9). This may result in initial rapid docking process that becomes irreversible and as a consequence kinetically inhibits the conformational reorganization in the locking phase. Even if the formation of amyloid fibrils is thermodynamically favored, the transition from monomers to amyloid conformations is kinetically limited 65 .

The other lysine residue, K28, is known to form important salt bridges in A $\beta$ fibrils 30,31 . Removing the positive charge on K28 by acetylation results in K28Ac peptide having longer lag time than WT A 342 during fibril formation. This is consistent with the stabilizing role of $\mathrm{K} 28$ salt bridge on the amyloid fibrils ${ }^{66}$. However, the K28Ac peptides were capable of forming amyloid structures upon longer incubation, either as pure K28Ac peptide sample, or in a heterogeneous equimolar mixture with WT A $\beta 42$ (Figure 2), 
suggesting K28 salt bridge may not play a role in the conformational transition from $\mathrm{A} \beta 42$ monomers to mature amyloids. This finding is also supported by a previously study of N$\varepsilon$-amino acetylation on K28 of A 25 -35 fragment, where the gross structure of A $\beta 25$ 35_K28Ac fibril were very similar to WT A $\beta 25-35$ fibrils ${ }^{67}$.

In this study we identified two major forms of aggregated structures: amorphous and fibrillar, from WT, acetylated $\mathrm{A} \beta 42$ peptides and their mixtures. The aggregates from these 7 different Aß42 peptides (WT, K16Ac, K28Ac, KKAc, and 1:1 mixtures of WT:K16Ac, WT:K28Ac, and WT:KKAc) can be further categorized based on their unique physicochemical properties as measured by fluorescent dyes, cell viability, ROS production, and their associated cytotoxicity (Table 1). WT A $\beta 42$ peptides $7 \mathrm{~d}$ aggregates show well organized fibrillar structure that has low flexibility, moderate ROS production, and high toxicity at $5 \mu \mathrm{M}$ concentrations (Figures 2,3,6B, C). K28Ac peptides aggregates form fibrils that are similar to the WT peptides but show high ROS production with high toxicity (Figures 2, 3; 6B, C). K16Ac peptides form aggregates that are amorphous, highly hydrophobic, and flexible (Figure 2, 3, 5). In addition, these aggregates also show cytotoxicity that is slightly higher than WT or K28Ac peptide with high ROS production (Figure $6 \mathrm{~B}, \mathrm{C}$ ). In case of neuronal cells, $7 \mathrm{~d}$ aggregates of $2 \mu \mathrm{M}$ WT A $\beta 42$ peptides and K28Ac show moderate ROS production but high cytotoxicity (Figure 7). In comparison, K16Ac peptides show much higher ROS production as well as cytotoxicity (Figure 7). Interestingly, $7 \mathrm{~d}$ aggregates from KKAc peptides that are amorphous in nature show significantly higher ROS production as well as cytotoxicity for SH-SY5Y as well as primary neuronal cells (Figures 6B, C, 7). Furthermore, the 1:1 mixtures of WT peptides 
with K16Ac, K28Ac, and KKAc, although have variable structural morphology, all show very high ROS production and cytotoxicity (Figures 2; 6B, C, 7), with WT: KKAc mixture showing the highest ROS production as well as cytotoxicity (Figure 6B, C). In addition, the KKAc peptides (pure or 1:1 mixture with WT A $\beta 42$ peptides) inhibit cell growth significantly (Figure 6A). The increased oxidative stress has been considered very important for pathogenesis in Alzheimer's disease as it can lead to oxidation of proteins, lipid peroxidation, free radical generation, mitochondrial dysfunction, and synaptic dysfunction ${ }^{68}$. And neurons are particular sensitive to ROS, which serves as source of oxidative stresses and results in damages in the brain ${ }^{60,69,70}$.

Table 1. Comparison of unique physicochemical properties of WT and acetylated A $\beta 42$ peptides and their mixtures for $7 \mathrm{~d}$ aggregates.

\begin{tabular}{|c|c|c|c|c|c|c|}
\hline $\begin{array}{c}\text { A } \beta 42 \\
\text { peptides }\end{array}$ & $\begin{array}{c}\text { Structure } \\
\text { type } \\
\text { (SEM) }\end{array}$ & $\begin{array}{c}\begin{array}{c}\text { Hydroph } \\
\text { obicity }\end{array} \\
\text { (ANS) }\end{array}$ & $\begin{array}{c}\text { Flexibilit } \\
y \\
\text { (Bis- } \\
\text { ANS) }\end{array}$ & $\begin{array}{c}\text { Cell viability } \\
\text { (MTS) }\end{array}$ & $\begin{array}{c}\begin{array}{c}\text { Cytotoxi } \\
\text { city }\end{array} \\
\text { (LDH) }\end{array}$ & $\begin{array}{c}\text { ROS } \\
\text { production } \\
\text { (DCF-HDA) }\end{array}$ \\
\hline WT & Fibril & + & + & $\begin{array}{l}\text { No } \\
\text { significant } \\
\text { inhibition }\end{array}$ & +++ & ++ \\
\hline K16Ac & $\begin{array}{c}\text { Amorphou } \\
\mathrm{S}\end{array}$ & ++++ & +++ & $\begin{array}{l}\text { No } \\
\text { significant } \\
\text { inhibition }\end{array}$ & +++ & +++ \\
\hline K28Ac & Fibril & ++ & + & $\begin{array}{l}\text { No } \\
\text { significant } \\
\text { inhibition }\end{array}$ & +++ & +++ \\
\hline KKAc & $\begin{array}{c}\text { Amorphou } \\
\mathrm{S}\end{array}$ & +++ & ++ & $\begin{array}{c}\text { Significant } \\
\text { Inhibition } \\
++\end{array}$ & ++++ & ++++ \\
\hline
\end{tabular}




\begin{tabular}{|l|c|c|c|c|c|c|}
\hline $\begin{array}{l}\text { WT }+ \\
\text { K16Ac } \\
(1: 1)\end{array}$ & $\begin{array}{c}\text { Amorphou } \\
\mathrm{s}\end{array}$ & ++ & + & $\begin{array}{c}\text { Significant } \\
\text { Inhibition }+\end{array}$ & +++ & +++ \\
\hline $\begin{array}{l}\text { WT }+ \text { K28 } \\
\text { Ac (1:1) }\end{array}$ & Fibril & ++ & + & $\begin{array}{c}\text { Significant } \\
\text { Inhibition }+\end{array}$ & +++ & +++ \\
\hline $\begin{array}{l}\text { WT }+ \\
\text { KKAc } \\
(1: 1)\end{array}$ & $\begin{array}{c}\text { Amorphou } \\
\mathrm{s}\end{array}$ & ++ & + & $\begin{array}{c}\text { Significant } \\
\text { Inhibition } \\
+++\end{array}$ & ++++ & ++++ \\
\hline
\end{tabular}

Number of + indicates proportional levels: $(+)=$ low; $(++)=$ moderate $;(+++)=$ high; $(++++)=$ highest among the group.

To visualize the effect of aggregated peptides we treated neuronal cells from rat with $2 \mu \mathrm{M}$ of $7 \mathrm{~d} A \beta 42$ peptides aggregates for $24 \mathrm{~h}$ (Figure 8). The neuronal cells treated with $\mathrm{A} \beta 42$ peptides for $24 \mathrm{~h}$, all show a significantly higher immunofluorescence signal for Tau in soma of neurons compared to neurites (Figure 8, Supplementary Figure 15). This is consistent with other literature reports where a direct relationship between amyloid $\beta$ oligomers leading to altered Tau protein biochemistry that may result in Tau that maybe fragmented, soluble, with/without requiring hyperphosphorylation and maybe toxic ${ }^{71-75}$ 1278.full;: Therefore, to better understand the oligomeric nature of $7 \mathrm{~d} A \beta 42$ peptides aggregates we analyzed by SDS-PAGE (Supplementary Figure 16). The $7 \mathrm{~d}$ peptides samples were run on a $15 \%$ SDS-gel and electrophoretically separated under fully denaturing conditions. These peptides largely separated in two groups, monomers and large higher molecular weight species with some oligomeric species seen for different A $\beta 42$ peptides as smear (Supplementary Figure 16). Although these $7 \mathrm{~d} A \beta 42$ peptides aggregates 
have distinct structural morphology as seen by SEM (Figure 2), on SDS-PAGE these largely show presence of monomeric and high molecular weight SDS-resistant species with some low molecular weight oligomeric species (Supplementary Figure 16). It is possible that cytotoxic effect of these peptides maybe mediated due to aberrant hydrophobic interaction directly or through $\mathrm{Tau}^{76,77} 71,73,78-80$.

Consistent with ROS production and cytotoxicity data of primary neuronal cells (Figure 7), immunostaining data showed significantly higher immunofluorescence signal for Tau in soma of neurons compared to neurites with the highest Tau signal for cells treated with KKAc peptides (Figure 8, Supplementary Figure 15). This is also consistent with other animal/cell model studies where increased cross talk between $A \beta$ and Tau has been reported with $A \beta$ exerting its toxicity via Tau that maybe fragmented, become oligomeric, with/without hyperphosphorylation and impact synaptotoxicity 74,75,81-84. Our data shows that the $A \beta 42$ peptides aggregates lead to increased Tau signal in soma of neuronal cells compared to control cells (Figure 8).

Although both K16Ac and KKAc aggregates are amorphous, the K16Ac aggregates are bead-like structures that are slightly more hydrophobic and flexible compared to KKAc (Figures 2, 3; 5). Both of these peptides $7 \mathrm{~d}$ aggregates show high toxicity but the difference in ROS production of these two peptides may be related to their subtle structure variations (Table 1$)^{78}$. This is consistent with recently reported literature findings where the toxicity of misfolded proteins was determined by both size and surface hydrophobicity of the molecule ${ }^{23}$. As large assemblies have lower surface hydrophobicity/volume ratios, they 
have lower diffusional mobility and lower potential to interact with cell membrane and receptors $^{23}$.

An alternative hypothesis suggests that the cytotoxicity may be related to the aggregation process that is dynamic, rather than a single structural species ${ }^{9,88}$. Minor shifts in the $A \beta 40$ : A $\beta 42$ ratio was demonstrated to be enough to modulate neurotoxicity ${ }^{89}$. The non-toxic $\mathrm{K} 16 \mathrm{~N}$ mutation of $\mathrm{A} \beta 42$ exhibits severe toxicity when mixed with WT peptides ${ }^{91}$. Here in this study, the aggregates formed from heterogeneous mixtures of WT and acetylated peptides all showed increased ROS production and toxicity compared to homogeneous samples of WT and K28Ac (Figures 6B, C, 7). Therefore, in addition to the structural characteristics, the dynamic interactions of different species contribute significantly to aggregation related toxicity.

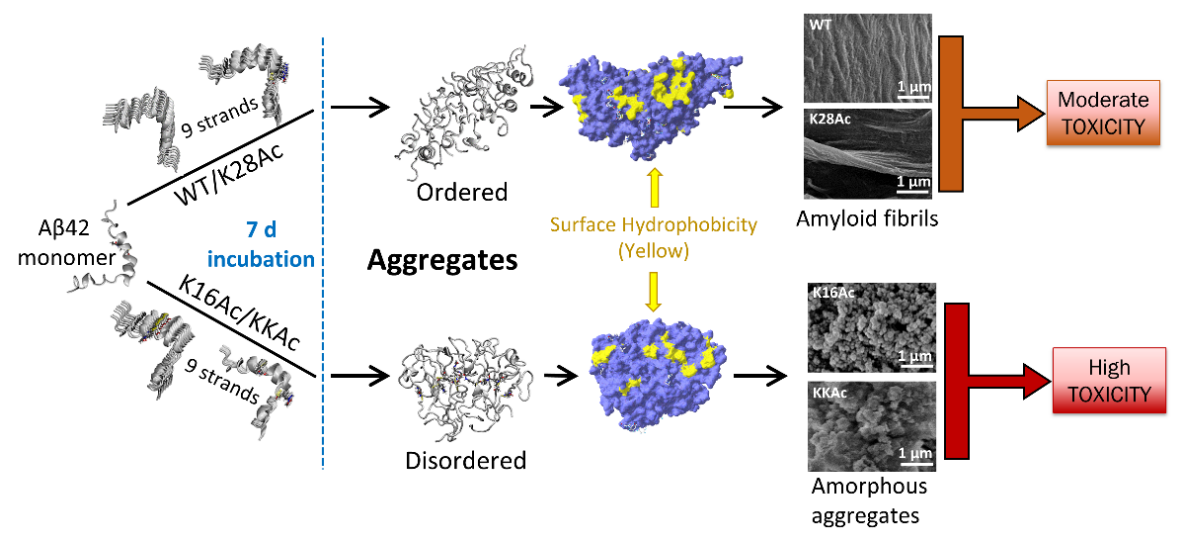

Figure 8. Suggested schematic model for WT and acetylation modified A $\beta 42$ peptides aggregation and cytotoxicity. WT A 342 and K28Ac peptides show a lag and log phase of aggregation kinetics and form ordered aggregates with large surface hydrophobic patches (yellow) that can assemble as amyloid fibrils and have moderate toxicity. Peptides 
acetylated at K16 (K16Ac or double acetylated KKAc) show rapid aggregation kinetics and form disordered, amorphous, and flexible aggregates that have higher surface hydrophobicity and high toxicity.

\subsection{Conclusion}

In this study, we focused on understanding how acetylation of A $\beta 42$ peptides (K16Ac, K28Ac, and KKAc) impacts its physicochemical properties and modulates its toxicity. For comparison WT A $\beta 42$ and 1:1 mixture of acetylated peptides with WT were also studied. As, acetylation plays an important role in protein folding and aggregation, understanding the effect of acetylation on $\mathrm{A} \beta 42$ peptides biophysical properties and biological health is critical to understanding its role in the disease process. Our study shows that although the structural morphology of peptides (WT, K16Ac, and KKAc) are different, all show increased cytotoxicity and ROS production. Compared to WT and K28Ac, all other peptides (K16Ac, KKAc, and 1:1 mixture of acetylated A $\beta$ with WT peptides) showed much higher increase in ROS production as well as cytotoxicity. Furthermore, the increased Tau signal in soma of neurons compared to control cells when treated with A $\beta 42$ peptides aggregates suggests a dynamic interaction between Tau and A $\beta 42$ peptides . Overall, the data suggests that in addition to the structural characteristics, the dynamic interactions of different $A \beta$ species may contribute significantly to toxicity directly or through cross-talk with other proteins such as Tau. 


\subsection{References}

1. Peters, C., et.al. Inhibition of amyloid beta-induced synaptotoxicity by a pentapeptide derived from the glycine zipper region of the neurotoxic peptide. Neurobiol Aging. 2013, 34(12):2805-14. doi: 10.1016/j.neurobio laging.2013.06.001.

2. Hernández, F., et.al. GSK3: a possible link between beta amyloid peptide and tau protein. Exp Neurol. 2010, 223(2):322-5. doi: 10.1016/j.expneurol.2009.09.011.

3. Assoc, A., Alzheimer's Association Report 2015 Alzheimer's disease facts and figures. Alzheimers \& Dementia 2015, 11, 332-384, doi:10.1016/j.jalz.2015.02.003.

4. Ballard, C., et al. Alzheimer's disease. Lancet 2011, 377, 1019-1031, doi:10.1016/S0140-6736(10)61349-9 (2011).

5. Kodali, R.; Williams, A. D.; Chemuru, S.; Wetzel, R., A beta(1-40) Forms Five Distinct Amyloid Structures whose beta-Sheet Contents and Fibril Stabilities Are Correlated. Journal of Molecular Biology 2010, 401, 503-517, doi:10.1016/j.jmb.2010.06.023.

6. Glabe, C. G., Structural Classification of Toxic Amyloid Oligomers. J Biol Chem 2008, 283, 29639-29643, doi:10.1074/jbc.R800016200.

7. Atwood, C. S., et al. Amyloid-beta: a chameleon walking in two worlds: a review of the trophic and toxic properties of amyloid-beta. Brain Research Reviews 2003, 43, 1-16, doi:10.1016/S0165-0173(03)00174-7. 
8. National Plan to Address Alzheimer's Disease: 2015 Update, U.S. Department of Health and Human Service (2015).

9. Hubin, E.; Van Nuland, N. A. J.; Broersen, K.; Pauwels, K.; Transient dynamics of A beta contribute to toxicity in Alzheimer's disease. Cellular and Molecular Life Sciences 2014, 71, 3507-3521, doi:10.1007/s00018-014-1634-z.

10. Honig, L. S., et al. Trial of Solanezumab for Mild Dementia Due to Alzheimer's Disease. New England Journal of Medicine 2018, 378, 321-330, doi:10.1056/NEJMoa1705971.

11. Kumar, S., et al. Phosphorylation of Amyloid-beta Peptide at Serine 8 Attenuates Its Clearance via Insulin-degrading and Angiotensin-converting Enzymes. J Biol Chem 2012, 287, 8641-8651, doi:10.1074/jbc.M111.279133.

12. Kumar, S., et al. Extracellular phosphorylation of the amyloid beta-peptide promotes formation of toxic aggregates during the pathogenesis of Alzheimer's disease. Embo Journal 2011, 30, 2255-2265, doi:10.1038/emboj.2011.138.

13. Kumar, S., et al. Early intraneuronal accumulation and increased aggregation of phosphorylated Abeta in a mouse model of Alzheimer's disease. Acta Neuropathologica 2013, 125, 699-709, doi:10.1007/s00401-013-1107-8.

14. Jarrett, J. T.; Berger, E. P.; Lansbury, P. T., The Carboxy Terminus of the BetaAmyloid Protein Is Critical for the Seeding of Amyloid Formation - Implications for the Pathogenesis of Alzheimers-Disease. Biochemistry 1993, 32, 4693-4697, doi:DOI 10.1021/bi00069a001. 
15. Sullivan, C. P., et al. Pyroglutamate-A beta 3 and 11 colocalize in amyloid plaques in Alzheimer's disease cerebral cortex with pyroglutamate-A beta 11 forming the central core. Neuroscience Letters 2011, 505, 109-112, doi:10.1016/j.neulet.2011.09.071.

16. Bouter, Y., et al. N-truncated amyloid beta (A beta) 4-42 forms stable aggregates and induces acute and long-lasting behavioral deficits. Acta Neuropathologica 2013, 126, 189-205, doi:10.1007/s00401-013-1129-2.

17. Murakami, K., et al. Isomerization and/or racemization at Asp23 of A beta 42 do not increase its aggregative ability, neurotoxicity, and radical productivity in vitro. Biochemical and Biophysical Research Communications 2008, 366, 745-751, doi:10.1016/j.bbrc.2007.12.009.

18. Shimizu, T.; Watanabe, A.; Ogawara, M.; Mori, H.; Shirasawa, T., Isoaspartate formation and neurodegeneration in Alzheimer's disease. Archives of Biochemistry and Biophysics 2000, 381, 225-234, doi:DOI 10.1006/abbi.2000.1955.

19. Jawhar, S.; Wirths, O.; Bayer, T. A., Pyroglutamate Amyloid-beta (A beta): A Hatchet Man in Alzheimer Disease. J Biol Chem 2011, 286, 38825-38832, doi:10.1074/jbc.R111.288308.

20. Ayyadevara, S., et al. Proteins that mediate protein aggregation and cytotoxicity distinguish Alzheimer's hippocampus from normal controls. Aging Cell 2016, 15, 924-939, doi:10.1111/acel.12501.

21. Tokikawa, R.; Loffredo, C.; Uemi, M.; Machini, M. T.; Bechara, E. J. H., Radical acylation of L-lysine derivatives and L-lysine-containing peptides by peroxynitrite- 
treated diacetyl and methylglyoxal. Free Radical Research 2014, 48, 357-370, doi:10.3109/10715762.2013.871386.

22. Dueramae, I.; Yoneyama, M.; Shinyashiki, N.; Yagihara, S.; Kita, R., Self assembly of acetylated dextran with various acetylation degrees in aqueous solutions: Studied by light scattering (vol 159, pg 171, 2017). Carbohydrate Polymers 2017, 161, 306-306, doi:10.1016/j.carbpol.2017.01.042.

23. Mannini, B., et al. Toxicity of Protein Oligomers Is Rationalized by a Function Combining Size and Surface Hydrophobicity. Acs Chemical Biology 2014, 9, 2309-2317, doi:10.1021/cb500505m.

24. Auburger, G.; Gispert, S.; Jendrach, M., Mitochondrial Acetylation and Genetic Models of Parkinson's Disease. Mitochondrion in Aging and Disease 2014, 127, 155-182, doi:10.1016/B978-0-12-394625-6.00006-4.

25. Srivastava, O. P. S., K.; Chaves, J.M.; Gill, A.K., Post-translationally modified human lens crystallin fragments show aggregation in vitro. Biochem Biophys Rep. 2017, 10, 94-131, doi:10.1016/j.bbrep.2017.01.011.

26. Drazic, A.; Myklebust, L. M.; Ree, R.; Arnesen, T., The world of protein acetylation. Biochimica Et Biophysica Acta-Proteins and Proteomics 2016, 1864, 1372-1401, doi:10.1016/j.bbapap.2016.06.007.

27. Gil, J.; Ramirez-Torres, A.; Encarnacion-Guevara, S., Lysine acetylation and cancer: A proteomics perspective. Journal of Proteomics 2017, 150, 297-309, doi:10.1016/j.jprot.2016.10.003. 
28. Ali, I.; Conrad, R. J.; Verdin, E.; Ott, M., Lysine Acetylation Goes Global: From Epigenetics to Metabolism and Therapeutics. Chemical Reviews 2018, 118, 340376, doi:10.1021/acs.chemrev.7b00181.

29. Morris, M., et al. Tau post-translational modifications in wild-type and human amyloid precursor protein transgenic mice. Nature Neuroscience 2015, 18, 1183-+, doi:10.1038/nn.4067.

30. Cook, C.; Stankowski, J. N.; Carlomagno, Y.; Stetler, C.; Petrucelli, L., Acetylation: a new key to unlock tau's role in neurodegeneration. Alzheimers Research \& Therapy 2014, 6, doi:ARTN 29 10.1186/alzrt259.

31. Gorsky, M. K.; Burnouf, S.; Dols, J.; Mandelkow, E.; Partridge, L., Acetylation mimic of lysine 280 exacerbates human Tau neurotoxicity in vivo. Scientific Reports 2016, 6, doi:ARTN 22685 10.1038/srep22685.

32. Xiao, Y. L., et al. A beta(1-42) fibril structure illuminates self-recognition and replication of amyloid in Alzheimer's disease. Nature Structural \& Molecular Biology 2015, 22, 499-U497, doi:10.1038/nsmb.2991.

33. Kajava, A. V.; Baxa, U.; Steven, A. C., beta arcades: recurring motifs in naturally occurring and disease-related amyloid fibrils. Faseb Journal 2010, 24, 1311-1319, doi:10.1096/fj.09-145979.

34. Ayyadevara, S., et al. Aspirin-Mediated Acetylation Protects Against Multiple Neurodegenerative Pathologies by Impeding Protein Aggregation. Antioxidants \& Redox Signaling 2017, 27, 1383-1396, doi:10.1089/ars.2016.6978. 
35. Tjernberg, L. O., et al. Arrest of beta-amyloid fibril formation by a pentapeptide ligand. J Biol Chem 1996, 271, 8545-8548, doi:DOI 10.1074/jbc.271.15.8545.

36. Roychaudhuri, R. et al, C-Terminal Turn Stability Determines Assembly Differences between A beta 40 and A beta 42. Journal of Molecular Biology 2013, 425, 292-308, doi:10.1016/j.jmb.2012.11.006.

37. Vetten, M. A., et al. Label-free in vitro toxicity and uptake assessment of citrate stabilised gold nanoparticles in three cell lines. Particle and Fibre Toxicology 2013, 10, doi:Artn 50 10.1186/1743-8977-10-50.

38. Aranda, A., et al. Dichloro-dihydro-fluorescein diacetate (DCFH-DA) assay: A quantitative method for oxidative stress assessment of nanoparticle-treated cells. Toxicology in Vitro 2013, 27, 954-963, doi:10.1016/j.tiv.2013.01.016.

39. Shan, Z.Y., et.al. Involvement of the Brain (Pro) renin Receptor in Cardiovascular Homeostasis. Circ Res 2010, 107, 934-+, doi: 10.1161/CIRCRESAHA.110.226977.

40. Huber, M.J., et.al. Increased activity of the orexin system in the paraventric ular nucleus contributes to salt-sensitive hypertension. 2017. 3(6):H1075-H1086. doi: 10.1152/ajpheart.00822.2016.

41. Schneider, C. A. R., W. S.; Eliceiri, K. W., "NIH Image to ImageJ: 25 years of image analysis". Nature methods 2012, 9, 671-675.

42. Jorgensen, W. L.; Chandrasekhar, J.; Madura, J. D.; Impey, R. W.; Klein, M. L., Comparison of Simple Potential Functions for Simulating Liquid Water. Journal of Chemical Physics 1983, 79, 926-935, doi:Doi 10.1063/1.445869. 
43. Miyamoto, S.; Kollman, P. A., Settle - an Analytical Version of the Shake and Rattle Algorithm for Rigid Water Models. Journal of Computational Chemistry 1992, 13, 952-962, doi:DOI 10.1002/jcc.540130805.

44. Feller, S. E.; Zhang, Y. H.; Pastor, R. W.; Brooks, B. R., Constant-Pressure Molecular-Dynamics Simulation - the Langevin Piston Method. Journal of Chemical Physics 1995, 103, 4613-4621, doi:Doi 10.1063/1.470648.

45. Darden, T.; York, D.; Pedersen, L., Particle Mesh Ewald - an N.Log(N) Method for Ewald Sums in Large Systems. Journal of Chemical Physics 1993, 98, 1008910092, doi:Doi 10.1063/1.464397.

46. Humphrey, W.; Dalke, A.; Schulten, K.; VMD: Visual molecular dynamics. Journal of Molecular Graphics \& Modelling 1996, 14, 33-38, doi:Doi 10.1016/0263-7855(96)00018-5.

47. Daggett, V., Molecular dynamics simulations of the protein unfolding/folding reaction. Accounts of Chemical Research 2002, 35, 422-429, doi:10.1021/ar0100834.

48. Brown, A. M.; Bevan, D. R., Molecular Dynamics Simulations of Amyloid betaPeptide (1-42): Tetramer Formation and Membrane Interactions. Biophys ical Journal 2016, 111, 937-949, doi:10.1016/j.bpj.2016.08.001.

49. Baumketner, A., et al. Amyloid beta-protein monomer structure: A computational and experimental study. Protein Science 2006, 15, 420-428, doi:10.1110/ps.051762406. 
50. Matthes, D.; Gapsys, V.; Brennecke, J. T.; De Groot, B. L., An Atomistic View of Amyloidogenic Self-assembly: Structure and Dynamics of Heterogeneous Conformational States in the Pre-nucleation Phase. Scientific Reports 2016, 6, doi:ARTN 33156 10.1038/srep33156.

51. Bitan, G., et al. Amyloid beta-protein (A beta) assembly: A beta 40 and A beta 42 oligomerize through distinct pathways. Proceedings of the National Academy of Sciences of the United States of America 2003, 100, 330-335, doi:10.1073/pnas.222681699.

52. $\mathrm{Xu}, \mathrm{Y}$. C., et al. Conformational transition of amyloid beta-peptide. Proceedings of the National Academy of Sciences of the United States of America 2005, 102, 5403-5407, doi:10.1073/pnas.0501218102.

53. Yang, M. F.; Teplow, D. B., Amyloid beta-Protein Monomer Folding: Free-Energy Surfaces Reveal Alloform-Specific Differences. Journal of Molecular Biology 2008, 384, 450-464, doi:10.1016/j.jmb.2008.09.039.

54. Phillips, J. C., et al. Scalable molecular dynamics with NAMD. Journal of Computational Chemistry 2005, 26, 1781-1802, doi:10.1002/jcc.20289.

55. MacKerell, A. D., et al. All-atom empirical potential for molecular modeling and dynamics studies of proteins. Journal of Physical Chemistry B 1998, 102, 35863616, doi:DOI 10.1021/jp973084f .

56. Urbanc, B. C.L.; Ding, F.; Sammond, D.; Khare, S.; Buldyrev, S.V.; Stanley, H.E.; Dokholyan, N.V., Molecular Dynamics Simulation of Amyloid $\beta$ Dimer Formation. Biophysical Journal 2004, 87, 2310-2321. 
57. Lijnzaad, P.; Argos, P., Hydrophobic patches on protein subunit interfaces: Characteristics and prediction. Proteins-Structure Function and Genetics 1997, 28, $333-343$.

58. Tonnies, E., Trushina, E., Oxidative Stress, Synaptic Dysfunction, and Alzheimer's Disease. Journal of Alzheimers Disease 2017, 57, 1105-1121, doi:10.3233/Jad161088.

59. Kamat, P. K., et al. Mechanism of Oxidative Stress and Synapse Dysfunction in the Pathogenesis of Altheimer's Disease: Understanding the Therapeutics Strategies. Molecular Neurobiology 2016, 53, 648-661, doi:10.1007/s12035-014-9053-6.

60. Angelova, P. R.; Abramov, A. Y., Alpha-synuclein and beta-amyloid different targets, same players: calcium, free radicals and mitochondria in the mechanism of neurodegeneration. Biochemical and Biophysical Research Communications 2017, 483, 1110-1115, doi:10.1016/j.bbrc.2016.07.103.

61. Liu, Z. W.; Zhou, T. Y.; Ziegler, A. C.; Dimitrion, P.; Zuo, L., Oxidative Stress in Neurodegenerative Diseases: From Molecular Mechanisms to Clinical Applications. Oxidative Medicine and Cellular Longevity 2017, doi:Artn 2525967 $10.1155 / 2017 / 2525967$

62. Smith, M. A.; Rottkamp, C. A.; Nunomura, A.; Raina, A. K.; Perry, G., Oxidative stress in Alzheimer's disease. Biochimica Et Biophysica Acta-Molecular Basis of Disease 2000,1502, 139-144, doi:Doi 10.1016/S0925-4439(00)00040-5. 
63. Zhao, Y., Zhao, B. L., Oxidative Stress and the Pathogenes is of Alzheimer's Disease. Oxidative Medicine and Cellular Longevity 2013, doi:Unsp 316523 $10.1155 / 2013 / 316523$

64. Chen, X. P.; Guo, C. Y.; Kong, J. M., Oxidative stress in neurodegenerative diseases. Neural Regeneration Research 2012, 7, 376-385, doi:10.3969/j.issn.16735374.2012.05.009.

65. Cheon, M., et al. Structural reorganisation and potential toxicity of oligomeric species formed during the assembly of amyloid fibrils. Plos Computational Biology 2007, 3, 1727-1738, doi:ARTN e173 10.1371/journal.pcbi.0030173.

66. Hartl, F. U.; Hayer-Hartl, M., Converging concepts of protein folding in vitro and in vivo. Nature Structural \& Molecular Biology 2009, 16, 574-581, doi:10.1038/nsmb.1591.

67. Kellermayer, M. S. Z., et al. Reversible mechanical unzipping of amyloid betafibrils. J Biol Chem 2005, 280, 8464-8470, doi:10.1074/jbc.M411556200.

68. Nguyen, P. H.; Li, M. S.; Stock, G.; Straub, J. E.; Thirumalai, D., Monomer adds to preformed structured oligomers of A beta-peptides by a two-stage dock-lock mechanism. Proceedings of the National Academy of Sciences of the United States of America 2007, 104, 111-116, doi:10.1073/pnas.0607440104.

69. Esler, W. P., et al. Alzheimer's disease amyloid propagation by a templatedependent dock-lock mechanism. Biochemistry 2000, 39, 6288-6295, doi:DOI 10.1021/bi992933h. 
70. Bernstein, S. L., et al. Amyloid-beta protein oligomerization and the importance of tetramers and dodecamers in the aetiology of Alzheimer's disease. Nature Chemistry 2009, 1, 326-331, doi:10.1038/Nchem.247.

71. Karsai, A., et al. Effect of lysine-28 side-chain acetylation on the nanomechanical behavior of Alzheimer amyloid beta 25-35 fibrils. Journal of Chemical Information and Modeling 2005, 45, 1641-1646, doi:10.1021/ci0501701.

72. Ahmad, W.; Ijaz, B.; Shabbiri, K.; Ahmed, F.; Rehman, S., Oxidative toxicity in diabetes and Alzheimer's disease: mechanisms behind ROS/RNS generation. Journal of Biomedical Science 2017, 24, doi:ARTN 76 10.1186/s12929-017-0379Z.

73. Uttara, B.; Singh, A. V.; Zamboni, P.; Mahajan, R. T., Oxidative Stress and Neurodegenerative Diseases: A Review of Upstream and Downstream Antioxidant Therapeutic Options. Current Neuropharmacology 2009, 7, 65-74, doi:Doi $10.2174 / 157015909787602823$.

74. Xie, H. R.; Hu, L. S.; Li, G. Y., SH-SY5Y human neuroblastoma cell line: in vitro cell model of dopaminergic neurons in Parkinson's disease. Chinese Medical Journal 2010, 123, 1086-1092, doi:10.3760/cma.j.issn.0366-6999.2010.08.021.

75. Rapoport, M.; Dawson, H. N.; Binder, L. I.; Vitek, M. P.; Ferreira, A., Tau is essential to beta-amyloid-induced neurotoxicity. Proceedings of the National Academy of Sciences of the United States of America 2002, 99, 6364-6369, doi:10.1073/pnas.092136199. 
76. Sato, C., et al. Tau Kinetics in Neurons and the Human Central Nervous System. Neuron 2018, 97, 1284-+, doi:10.1016/j.neuron.2018.02.015.

77. Reifert, J.; Hartung-Cranston, D.; Feinstein, S. C., Amyloid beta-Mediated Cell Death of Cultured Hippocampal Neurons Reveals Extensive Tau Fragmentation without Increased Full-length Tau Phosphorylation. J Biol Chem 2011, 286, 2079720811, doi:10.1074/jbc.M111.234674.

78. Bloom, G. S., Amyloid-beta and Tau The Trigger and Bullet in Alzheimer Disease

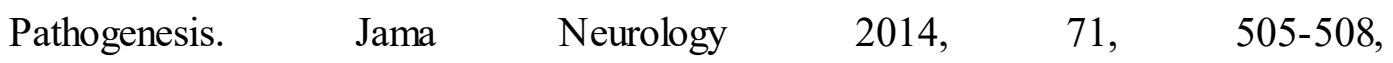
doi:10.1001/jamaneuro1.2013.5847.

79. Nisbet, R. M.; Polanco, J. C.; Ittner, L. M.; Gotz, J., Tau aggregation and its interplay with amyloid-beta. Acta Neuropathologica 2015, 129, 207-220, doi:10.1007/s00401-014-1371-2.

80. Pryor, N. E.; Moss, M. A.; Hestekin, C. N., Unraveling the Early Events of Amyloid-beta Protein (A beta) Aggregation: Techniques for the Determination of A beta Aggregate Size. Int J Mol Sci 2012, 13, 3038-3072, doi:10.3390/ijms13033038.

81. Jan, A.; Gokce, O; Luthi-Carter, R.; Lashuel, H. A., The ratio of monomeric to aggregated forms of A beta 40 and $\mathrm{A}$ beta 42 is an important determinant of amyloid-beta aggregation, fibrillogenesis, and toxicity. J Biol Chem 2008, 283, 28176-28189, doi:10.1074/jbc.M803159200.

82. Hoshi, M., et al. Spherical aggregates of beta-amyloid (amylospheroid) show high neurotoxicity and activate tau protein kinase I/glycogen synthase kinase-3 beta. 
Proceedings of the National Academy of Sciences of the United States of America 2003, 100, 6370-6375, doi:10.1073/pnas.1237107100.

83. Yu, Y.; Jans, D. C.; Winblad, B.; Tjernberg, L. O.; Schedin-Weiss, S., Neuronal A beta 42 is enriched in small vesicles at the presynaptic side of synapses. Life Science Alliance 1 2018, doi:ARTN e201800028 10.26508/lsa.201800028 .

84. Funke, S., Detection of Soluble Amyloid- $\beta$ Oligomers and Insoluble HighMolecular-Weight Particles in CSF: Development of Methods with Potential for Diagnosis and Therapy Monitoring of Alzheimer's Disease. International Journal of Alzheimer's disease 2011, 1-8, doi:10.4061/2011/151645.

85. Small, S. A.; Duff, K.; Linking A beta and Tau in Late-Onset Alzheimer's Disease: A Dual Pathway Hypothesis. Neuron 2008, 60, 534-542, doi:10.1016/j.neuron.2008.11.007.

86. Nieweg, K.; Andreyeva, A.; Van Stegen, B.; Tanriover, G.; Gottmann, K., Alzheimer's disease-related amyloid-beta induces synaptotoxicity in human iPS cell-derived neurons. Cell Death \& Disease 2015, 6, doi:ARTN e1709 10.1038/cddis.2015.72.

87. Rajmohan, R. \& Reddy, P. H. Amyloid-Beta and Phosphorylated Tau Accumulations Cause Abnormalities at Synapses of Alzheimer's disease Neurons. Journal of Alzheimers Disease 2017, 57, 975-999, doi:10.3233/Jad-160612.

88. Guerrero-Munoz, M. J.; Gerson, J.; Castillo-Carranza, D. L., Tau Oligomers: The Toxic Player at Synapses in Alzheimer's Disease. Frontiers in Cellular Neuroscience 2015, 9, doi:ARTN 464 10.3389/fncel.2015.00464. 
89. Ross, C. A.; Poirier, M. A., What is the role of protein aggregation in neurodegeneration? Nature Reviews Molecular Cell Biology 2005, 6, 891-898, doi:DOI $10.1038 / \mathrm{nrm} 1742$.

90. Pauwels, K., et al. Structural Basis for Increased Toxicity of Pathological A beta(42):A beta(40) Ratios in Alzheimer Disease. J Biol Chem 2012, 287, 56505660, doi:10.1074/jbc.M111.264473 .

91. Kaden, D., et al. Novel APP/A ss mutation K16N produces highly toxic heteromeric A ss oligomers. Embo Molecular Medicine 2012, 4, 647-659, doi:10.1002/emmm.201200239. 


\section{Chapter 5: Summary and Future Perspective}

Protein folding, misfolding, and aggregation are at the core of proteinopathies and have been studied extensively. How protein aggregates morphology and its unique physicochemical properties are linked to cellular toxicity is still a subject of intense debate. There are substantial number of published studies that have focused on fibrillar structure of proteins, with very few studies exploring the role of amorphous protein aggregate structures in the disease process. In this dissertation, we focused on two proteins that are very important from a neurodegenerative perspective: 1) Role of acetylation of amyloid $\beta$ 42 on cytotoxicity and 2) Expression, purification, and characterization of Tar-DNA binding protein 43 (TDP-43) from a eukaryotic source. Both of these studies are very important as role of amyloid $\beta$ in Alzheimer's toxicity has long been known but still the toxic form/fold of $A \beta$ is not well understood. Moreover, role posttranslational modification such as acetylation may play in the disease process is still not very well understood and is a topic of intense debate. For the TDP43 project, the importance of this protein has been shown for several neurodegenerative diseases such as ALS, Alzheimer's, and Parkinson's disease and the literature is filled with biochemical and biophysical characterization from prokaryotic sources with very little work done using proteins from eukaryotic source. Therefore, in this dissertation I tried to shed light on the role, acetylation of A 42 (K16Ac, K28Ac, and KKAc) have on amyloid $\beta$ toxicity, and also expressed and purified TDP43 protein from insect cells (eukaryotic source) and studied their basic biochemical and biophysical properties. 
We chose to investigate the effect acetylation of $A \beta$ peptide have on modulating cellular toxicity, as these have major implication for pathogenes is in Alzheimer's disease. Analys is of physicochemical properties of acetylated peptides showed that amorphous structures play a critical role in proteins physicochemical properties and its associated toxicity. This work underscores the importance of posttranslational modification such as acetylation of $\mathrm{A} \beta$ peptides on toxicity as well as aggregate morphology (e.g. K16Ac has amorphous structure) which will lead to better drug designs in future.

Next, we standardized the procedure for purification of TDP- 43 protein, now known to be involved in several neurodegenerative diseases like ALS, FTLD, Alzheimer's, Lewy body, and Huntington. Even though, several elegant research studies have been conducted on this protein using prokaryotic proteins, a method for purifying full-length protein using a eukaryotic expression system is lacking. Furthermore, purification of full-length protein is challenging as its overexpression leads to aggregation. And for the same reasons, detailed biochemical and biophysical studies on full-length protein from eukaryotic source and its associated toxicity are lacking. We have standardized the procedure for purifying fulllength TDP-43 protein using a eukaryotic expression system that is rigorous but reproducible. Furthermore, we investigated the morphology of TDP-43 protein aggregates under disulfide reducing and non-reducing conditions and found these form amorphous structures. This opens-up possibility of future studies on exploring the role sulfhydryl status of the protein plays in the toxicity of these proteins and hence the disease process.

TDP-43 has six sulfhydryl residues (Cys) which opens up the possibility of disulfide bond formation that maybe inter-chain or intra-chain. Our initial experiment on TDP-43 full155 
length protein using mal-PEG (5KDa) shows some shift in the band of proteins suggesting susceptibility of TDP-43 to disulfide reducing agent. How these Cys residues may play a role in aggregation and cellular toxicity needs to be explored further keeping in view the redox status of cells and how this equilibrium can shift under cellular stress.

There has been a renewed interest in determining the toxic fold of a protein to better understand its role in protein misfolding, aggregation, and its toxicity. This is where having aggregates that are well characterized from a eukaryotic source for TDP-43 protein will be of great interest to researchers trying to understand role of TDP- 43 protein in the disease process. This is because a protein from eukaryotic source will have all natural posttranslational modification that are expected in the mature protein. While, studies on TDP-43 proteins from bacterial source have contributed significantly to scientific literature, it may still miss out some key findings that may be linked to its natural posttranslational modifications. In addition, a methodical study exploring morphology and physicochemical properties of TDP-43 full-length aggregates under reducing and nonreducing cellular environment are lacking. Hence, we made an attempt to study the nature of aggregates of TDP-43 at physiological temperature in presence and absence of reducing agent like TCEP. Our results show that TDP-43 full length protein forms amorphous aggregates. These aggregates need to be further explored to investigate their associated toxicity using neuronal cell lines for biological relevance. These can be some of the same assays as outlined in amyloid $\beta$ toxicity chapter such as MTS, DCF-HDA, and LDH assay.

The future studies can explore two areas of research that are intricately related to my $\mathrm{PhD}$ work: 1) Role of posttranslational modification such as acetylation, phosphorylation, in the 156 
disease process (stems from $A \beta$ acetylation study), 2) Do TDP-43 protein from a eukaryotic source may have some unique characteristics that the prokaryotic protein does not have. Future studies on these can help better understand the toxic fold/nature of proteins and can help shed light on the pathogenesis in the disease process. Eventually, these studies may lead to development of better and more effective drugs. 


\section{Appendix A Supporting Information for Chapter 3}

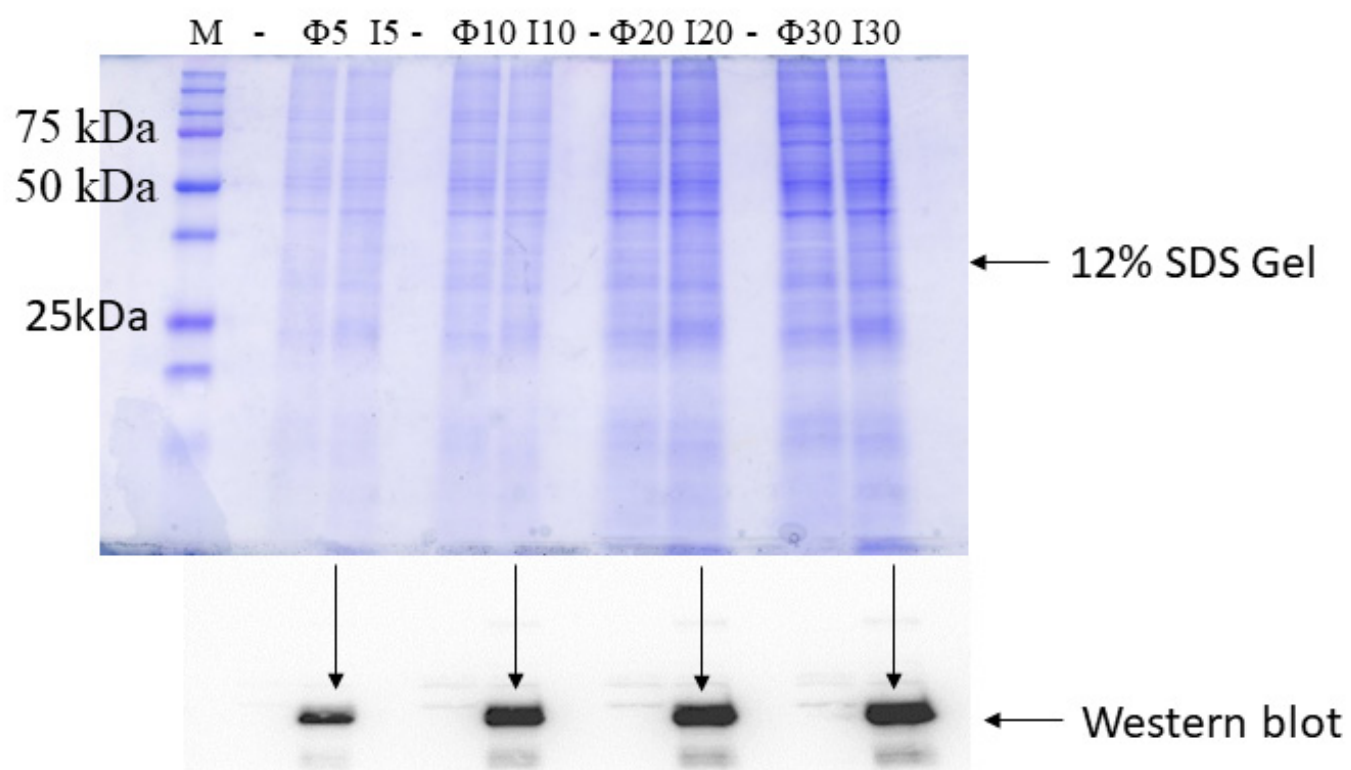

Figure S1: Reducing SDS PAGE and Western blot showing the expression of TDP-43 full length protein. M-Marker, $\Phi$ (Uninfected lysate), I (Infected lysate). Uninfected or infected lysate was loaded $5,10,20$ and $30 \mu \mathrm{g} / \mathrm{lane}$ represented as $\Phi 5, \Phi 10, \Phi 20, \Phi 30$ on $12 \%$ SDS PAGE. The gels were run for 3 hours. For Western blot, the blot was incubated with Anti-TDP-43 (N-terminal region) produced antibody (1:4000) and Goat anti-rabbit IgGHRP (1:10000). 


\section{Ammonium sulfate precipitation:}

Ammonium sulfate precipitation is one of the common method employed in purification of a protein. It is based on the principle of salting out. Different percentage of saturated ammonium sulfate solution was used for protein extraction. The schematic representation for ammonium sulfate precipitation is shown below in figure S2. In brief, the cell pellets were first lysed in lysis buffer and centrifuged at $20,000 \mathrm{~g}$ for 1 hour at $4^{0} \mathrm{C}$. The resultant supernatant was then subjected to slow addition of ammonium sulfate to make it $30 \%$ saturated. The saturated solution was subjected to centrifugation at $20,000 \mathrm{~g}$ for 1 hour at $4^{0} \mathrm{C}$. The supernatant obtained in this step was again subjected to ammonium sulfate to get $30 \%$ saturated solution. The resultant solution was then centrifuged at same speed, time and temperature. The supernatant thus obtained from this step was again subjected to ammonium sulfate to get $50 \%$ saturated solution. Then, after $70 \%$ saturated solution was subjected to centrifugation at $20,000 \mathrm{~g}$ for 1 hour at $4^{0} \mathrm{C}$. The supernatant and pellet obtained in each step was saved and tested in western blot. The procedure for SDS PAGE and Western blot was followed similarly as explained in material and methods in Chapter 3. As shown in figure S3, the protein bands were observed in Supernatant obtained after cell lysis and $30 \%$ ammonium sulfate precipitation represented by S0 and S30 respectively. However, no clear bands were observed in supernatant obtained after 50 and 70\% (S50 and S70) ammonium sulfate precipitation. Similarly, the total pellet (P0) and pellet obtained after 30\% ammonium sulfate precipitation (P30) showed the significant amount of protein and to some extent in pellet obtained after 50\% ammonium sulfate precipitation (P50). But no band was observed in pellet obtained after $70 \%$ ammonium sulfate precipitation $(\mathrm{P} 70)$. 
Based on finding from western blot we decided to run the fractions S0, S30 and P30 in SDS PAGE and Western blot. We used BCA assay to estimate the protein concentration

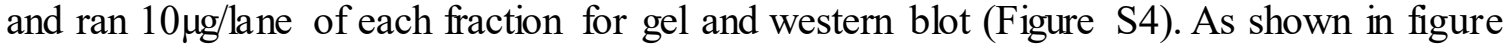
S4, we observed the protein bands at our desired molecular weight $(\sim 50 \mathrm{kDa})$ in all three samples but while comparing SDS PAGE we did not observe the concentrated band for protein at $50 \mathrm{KDa}$ instead a smear/multiple bands were observed in all three samples suggesting of protein mixtures.

\section{Ammonium sulfate precipitation of lysates:}

Pellets stored at $-80^{\circ} \mathrm{C}$

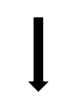

Resuspend in Lysis buffer (work on ice)

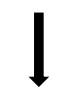

Homogenize using bead beater for 12 cycles

Centrifuge the lysates at 20,000g/maximum possible speed for 1 hour at $4^{0} \mathrm{C}$

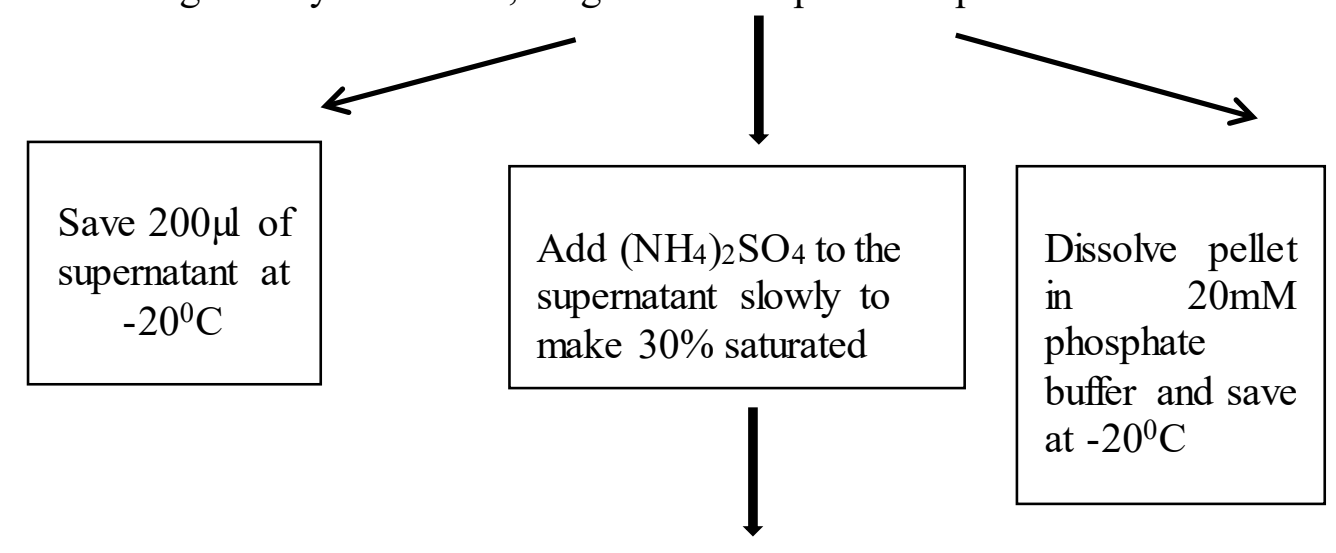


Centrifuge at $20,000 \mathrm{~g}$ for 30 minutes at $4^{0} \mathrm{C}$
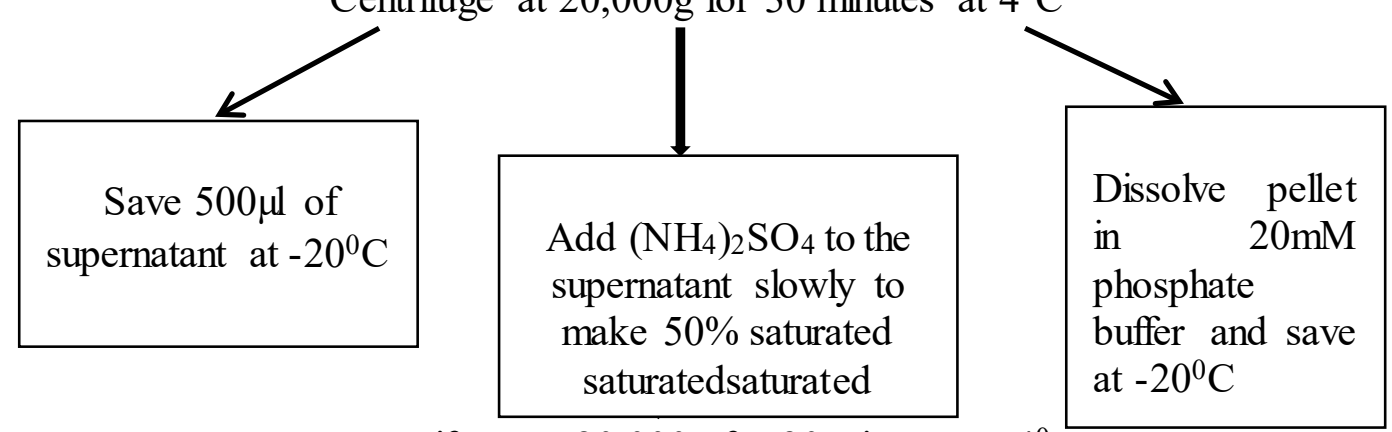

Centrifuge at $20,000 \mathrm{~g}$ for 30 minutes at $4^{0} \mathrm{C}$

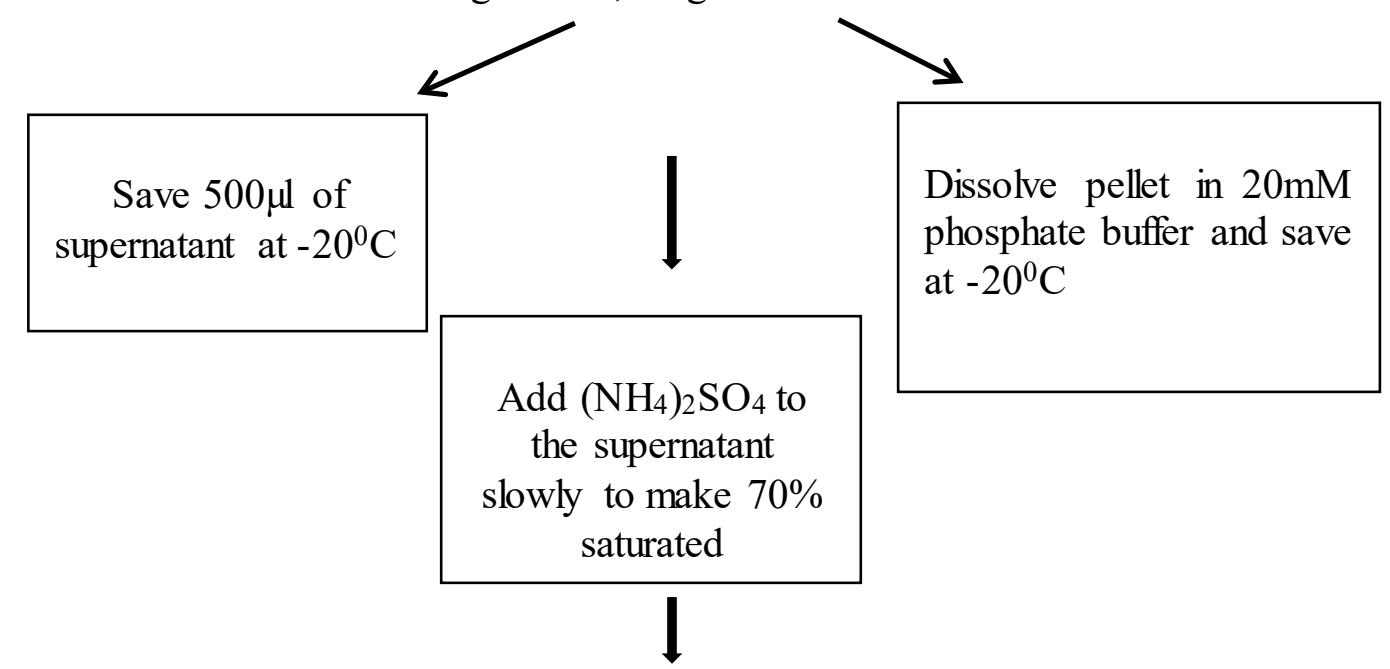

Centrifuge at $20,000 \mathrm{~g}$ for 30 minutes at $4^{0} \mathrm{C}$
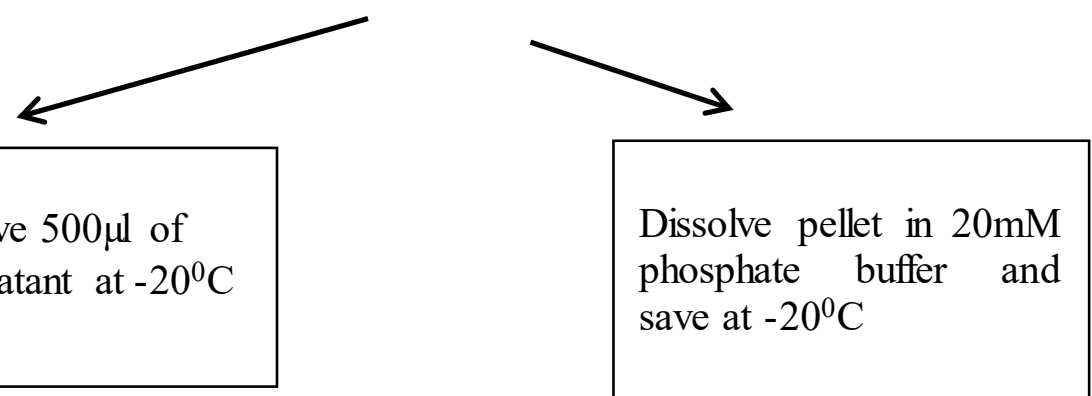

Save $500 \mu \mathrm{l}$ of supernatant at $-20^{\circ} \mathrm{C}$ phosphate buffer and save at $-20^{\circ} \mathrm{C}$ 


\section{Note:}

To each $500 \mu \mathrm{l}$ of supernatant saved after $30 \%, 50 \%$ and $70 \%$ saturation with $\left(\mathrm{NH}_{4}\right)_{2} \mathrm{SO}_{4}$ carry out following protocol:

$$
500 \mu \text { of supernatant }
$$

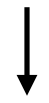

Dilute by $50 \%$ in autoclaved double distilled water

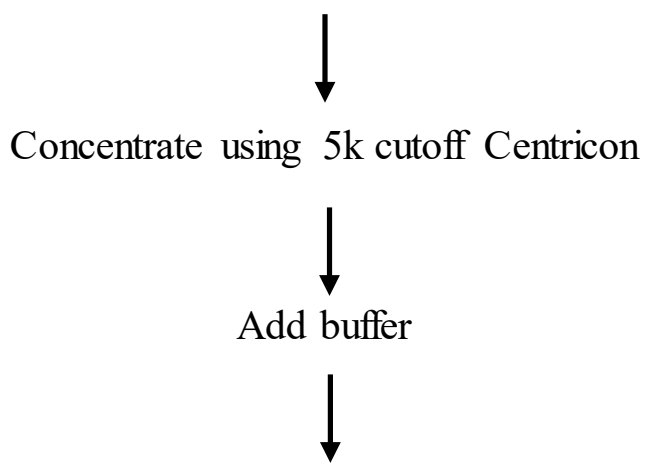

Concentrate using $5 \mathrm{k}$ centricon and make the volume around $200 \mu \mathrm{l}$ to $250 \mu \mathrm{l}$. (Save at $\left.20^{\circ} \mathrm{C}\right)$

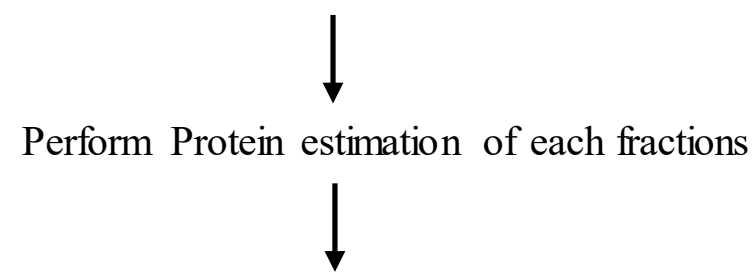

Run the SDS- PAGE

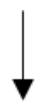

Western blot

Figure S2: Schematic representation of Scheme for Ammonium Sulfate Precipitation 


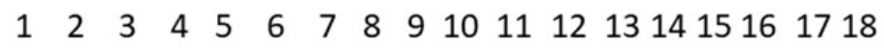

$M \phi S 0 \phi S 30 \phi S 50 \phi S 70 \phi P 0 \phi P 30 \phi P 50 \phi P 70 M$
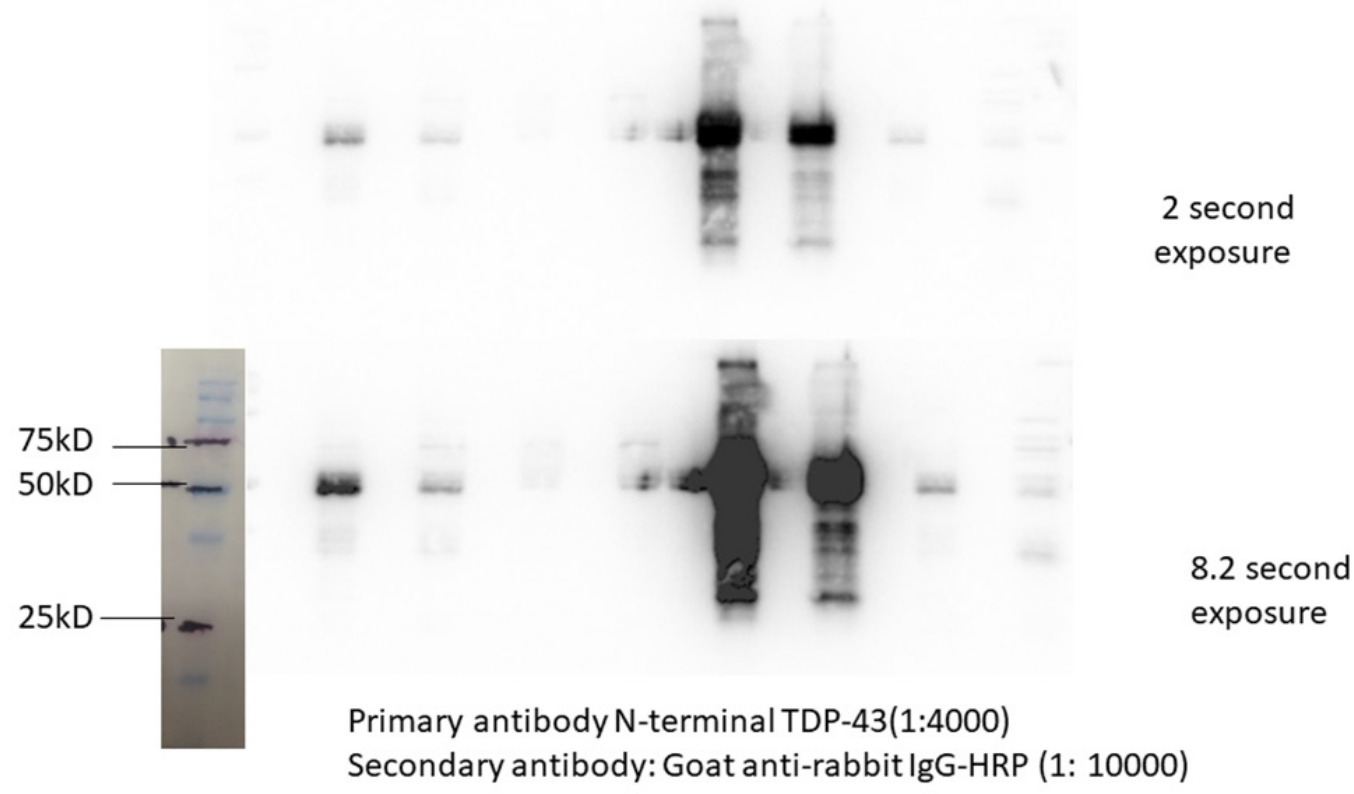

Figure S3: Western blot of supernatant and pellet fractions obtained after different percentage of ammonium sulfate precipitation. The notation in the figure represents as follows: M-Marker, S0- Supernatant obtained after cell lysis, S30, S50 and S70Supernatant obtained after 30,50 and 70\% ammonium sulfate precipitation and $\mathrm{P} 0$-pellet obtained after cell lysis, P30, P50 and P70-Pellet obtained after 30, 50 and 70\% ammonium sulfate precipitation. The blot was incubated with primary antibody N-terminal TDP-43 (1:4000) and secondary antibody, Goat anti-rabbit IgG-HRP (1:10000) 


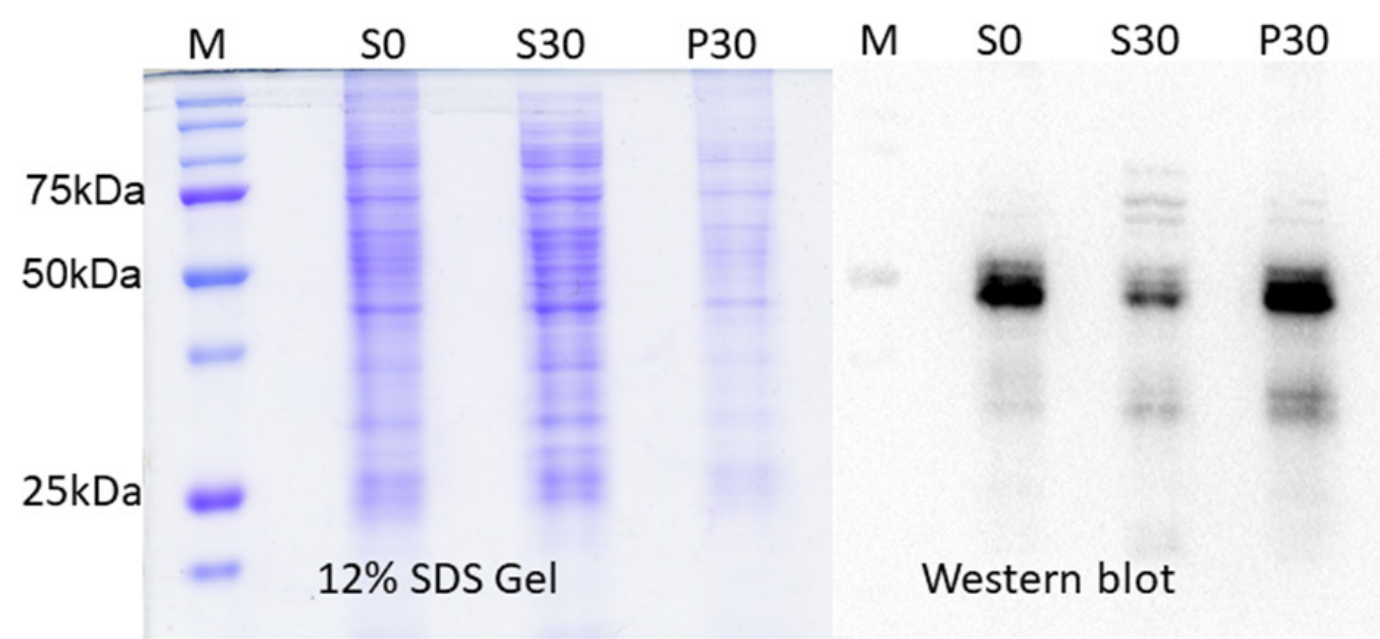

Figure S4: SDS Page and Western blot of S0, S30 and P30 fractions.

Next, we decided to proceed the NI-NTA purification using the pellet obtained after $30 \%$ ammonium sulfate precipitation ( $\mathrm{P} 30)$. The pellet obtained was dissolved in phosphate buffer and stored at $-20^{\circ} \mathrm{C}$. Then, on the day of purification, the pellet was diluted in equilibration buffer (20mM sodium phosphate, $300 \mathrm{mM} \mathrm{NaCl}$ (PBS) containing $10 \mathrm{mM}$ imidazole, pH 7.4) and loaded on the packed Ni-NTA column. The bound flow through was collected after loading the dissolved pellet on the column. The column was washed with wash buffer (PBS with $25 \mathrm{mM}$ imidazole, $\mathrm{pH}$ 7.4) for 3-5 column volume and wash flow through was collected to remove the unbound proteins. Finally, the elution fractions were collected in microcentrifuge tubes using elution buffer (PBS containing 0.5M imidazole, $\mathrm{pH}$ 7.4). After the column purification Western blot of elution fractions were performed to determine the elution fractions containing the protein (Figure S5-S6). Figure S5 represents the Ni-NTA carried out with only small portion of pellet. As shown in figure S6, the elution fractions from tube number 1 to13 shows the presence of TDP-43 protein 
in good amount. However, a good amount of protein was obtained in bound flow through and wash flow through.

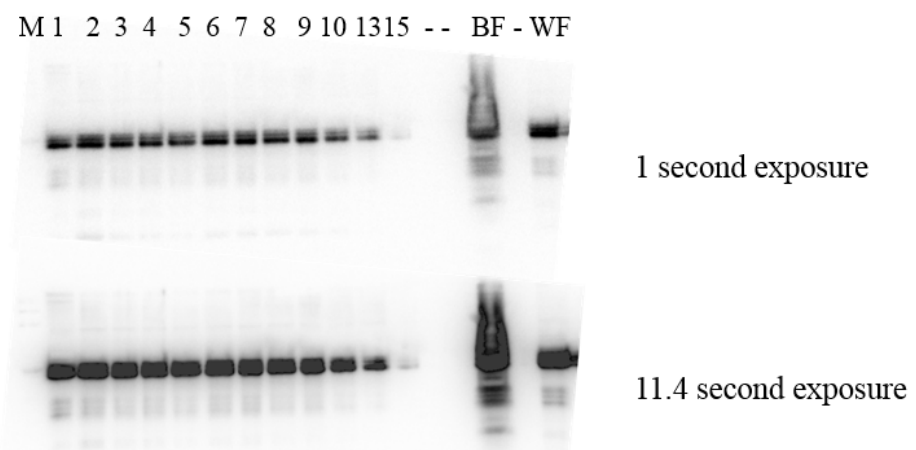

All samples loaded $25 \mu 1$

$1,2,3,4,5,6,7,8,9,10,13,15$ - Different Elution fractions

BF- Bound flow through

WF-Wash flow through

Fig S5: Western blot result of P30 after Ni-NTA column (Carried out with larger portion of pellet) 


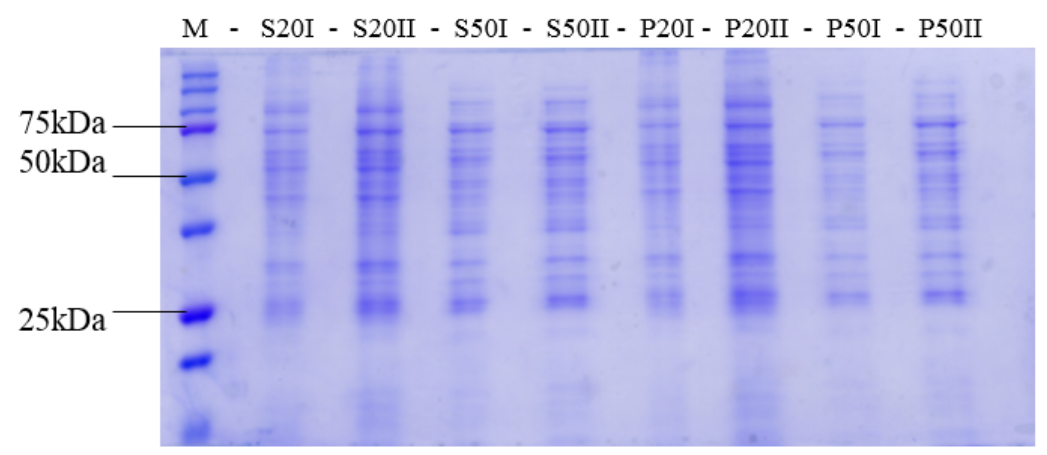

$12 \%$ Gel , 80V, 3 hours

S20I, S20II- Supernatant obtained after $20 \%$ ammonium sulfate precipitation loaded 10 and $20 \mu \mathrm{g} /$ lane respectively.

S50I, S50II- Supernatant obtained after 50\% ammonium sulfate precipitation loaded 10 and $20 \mu \mathrm{g} /$ lane respectively.

P20I, P20II- Pellet obtained after 20\% ammonium sulfate precipitation loaded 10 and 20 $\mu \mathrm{g} /$ lane respectively.

P50I, P50II- Pellet obtained after 50\% ammonium sulfate precipitation loaded 10 and 20 $\mu \mathrm{g} /$ lane respectively.

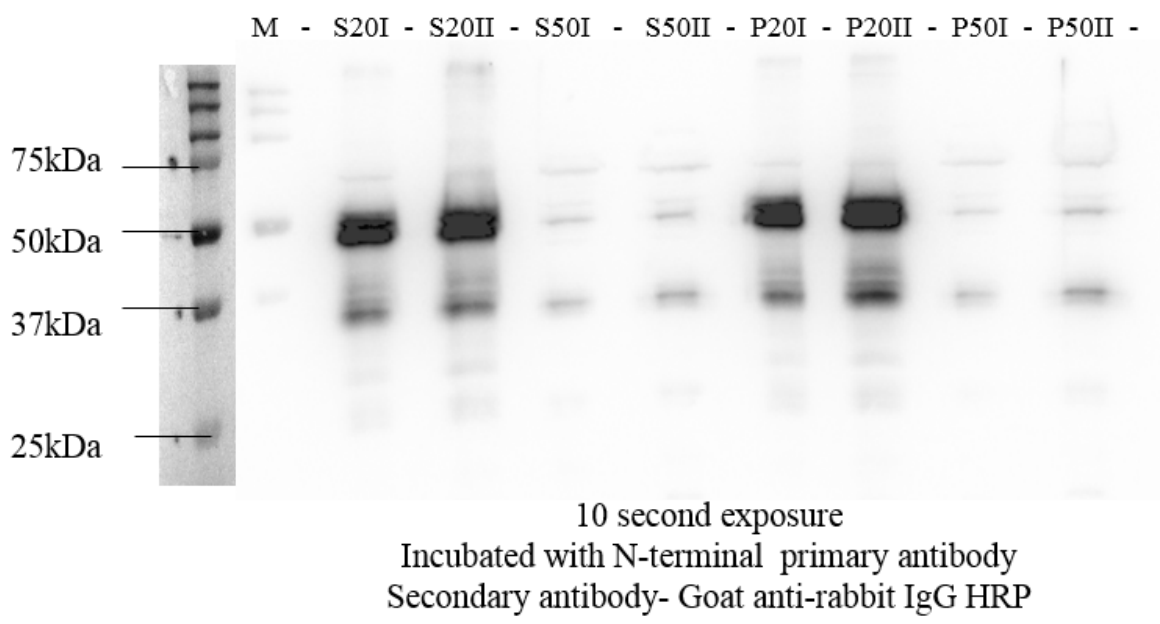

Fig S6: SDS PAGE and Western blot results of Pellet and supernatant fractions of total lysates after 20 and $50 \%$ ammonium sulfate precipitation. 


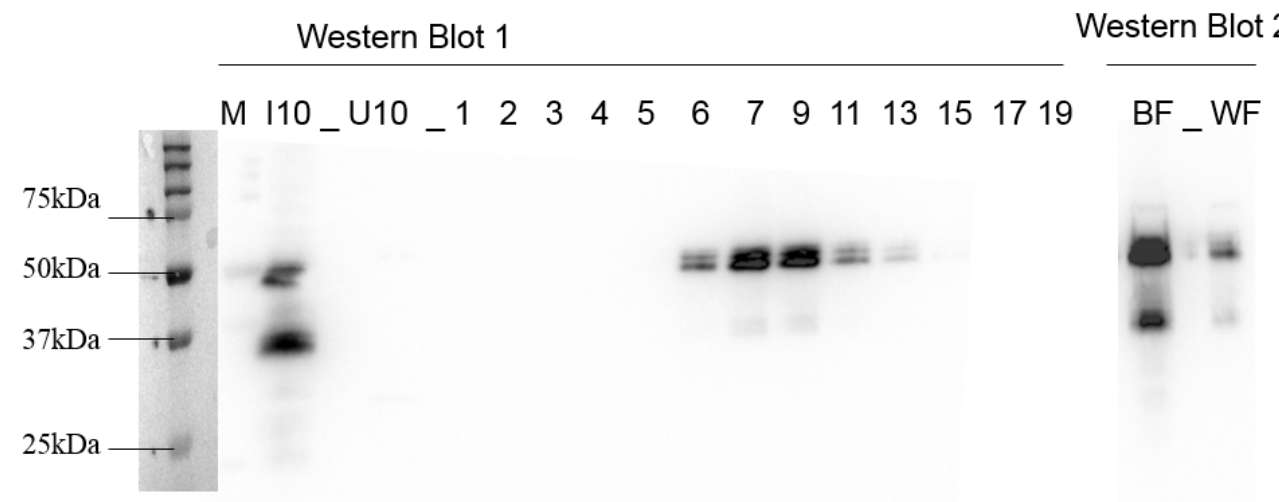

17.6 second exposure

Incubated with N-terminal primary antibody

Secondary antibody- Goat anti-rabbit IgG HRP

Total lysate(I10) and control lysate (U10) loaded $10 \mu \mathrm{g} /$ lane and for all other elution fractions including bound and wash flow through $10 \mu \mathrm{l}$ of sample was taken and mixed with $10 \mu \mathrm{l}$ of deionized water and finally mixed with $10 \mu \mathrm{l}$ of $3 \mathrm{X}$ sample buffer .

Fig S7: Western blot results of P20 after Ni-NTA column. (TDP-43 full length)

\section{SDS PAGE and Western blot results of detergent soluble fractions from total pellet} (P0).

After ammonium sulfate precipitation, we next tried to dissolve our protein using detergent $(0.5 \% \mathrm{SDS})$. For this purpose, to the pellet obtained after cell lysis phosphate buffer was added and then $0.5 \%$ SDS was added. The pellet was allowed to incubate at $4{ }^{0} \mathrm{C}$ for overnight in a shaker. Next day, the soluble fraction was obtained by centrifugation and protein estimation of detergent soluble fraction was carried out by BCA assay. The insoluble fraction was dissolved in $20 \mathrm{mM}$ phosphate buffer and stored at $-20^{\circ} \mathrm{C}$. SDS PAGE and western blot for detergent soluble and insoluble fractions were carried out using same primary and secondary antibody used for N-terminal TDP-43. SDS-PAGE and Western blot of soluble fraction was carried out at different concentrations $(1,2,5$ and $10 \mu \mathrm{g} / \mathrm{lane})$. Applying this procedure, we could see the bands for TDP-43 with increasing 
concentration as loaded but some additional bands were observed at higher exposure of blot in western blot imaging which is suggestive of some degraded products. Further, while

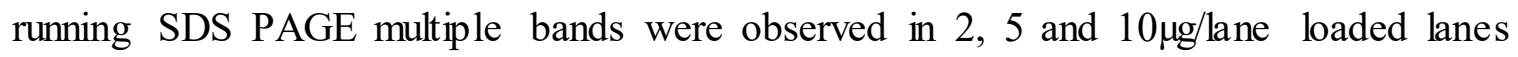
suggestive of protein mixtures. Also, no darker bands were observed towards our expected molecular weight .

The schematic representation of procedure is shown below:

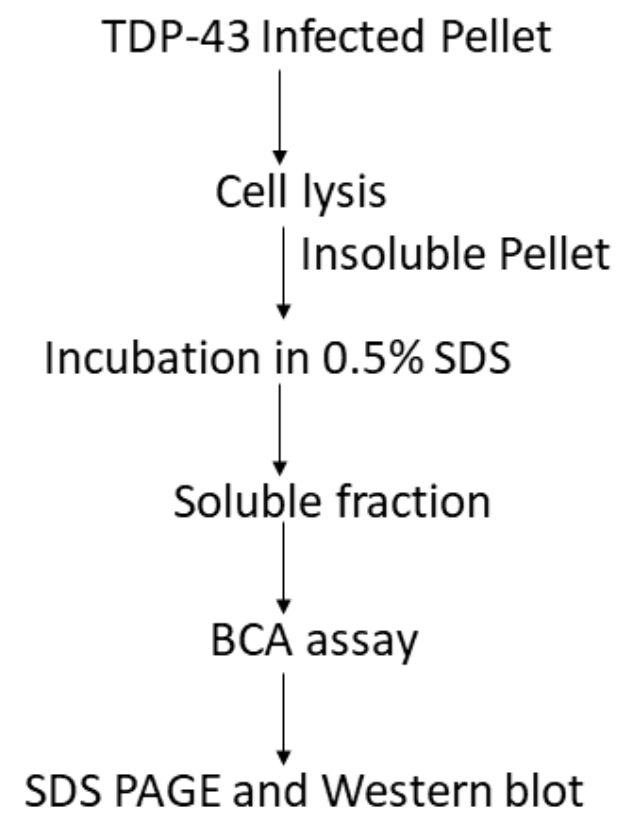




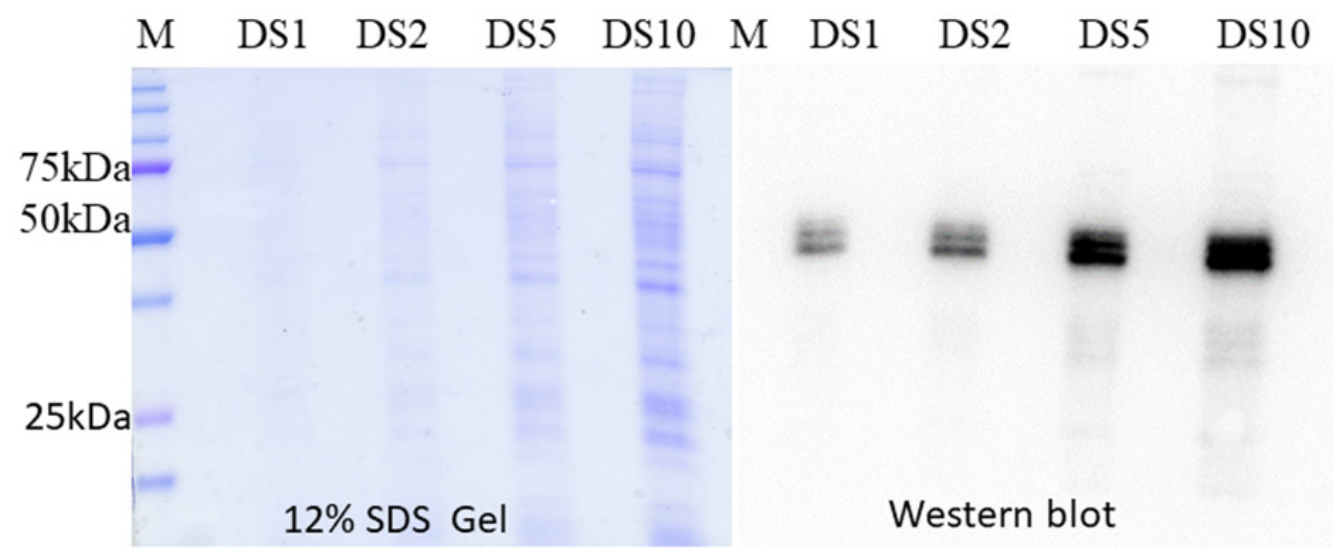

Figure S8: Reducing SDS PAGE and Western blot showing the TDP-43 protein level in detergent soluble fraction. Detergent soluble fraction (DS) was loaded at different volumes $1 \mu \mathrm{g} / \mathrm{lane}, 2 \mu \mathrm{g} / \mathrm{lane}, 5 \mu \mathrm{g} / \mathrm{lane}$ and $10 \mu \mathrm{g} / \mathrm{lane}$ represented as DS1, DS2, DS5 and DS10.

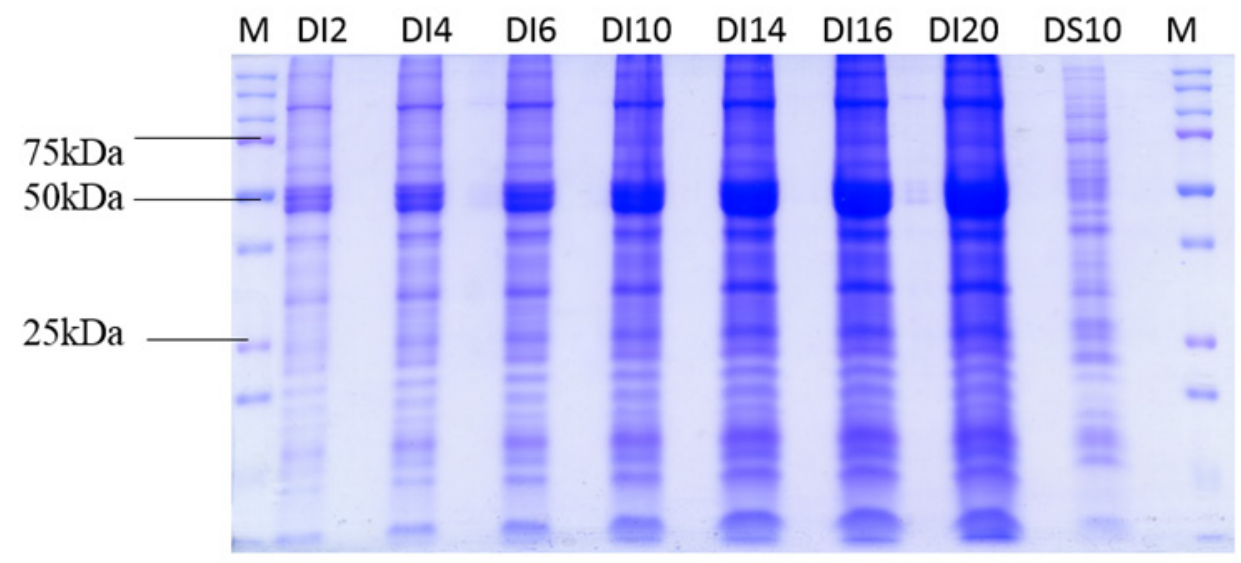

Figure S9: Reducing SDS PAGE showing the TDP-43 protein level in detergent insoluble fraction. Detergent insoluble fraction (DI) was loaded at different volumes $1,4,6,10,14$, 16 and 20ul, represented as DI2, DI4, DI6, DI10, DI14, DI 16, DI20 and detergent soluble fraction loaded $10 \mu \mathrm{g} /$ lane represented as DS10. 


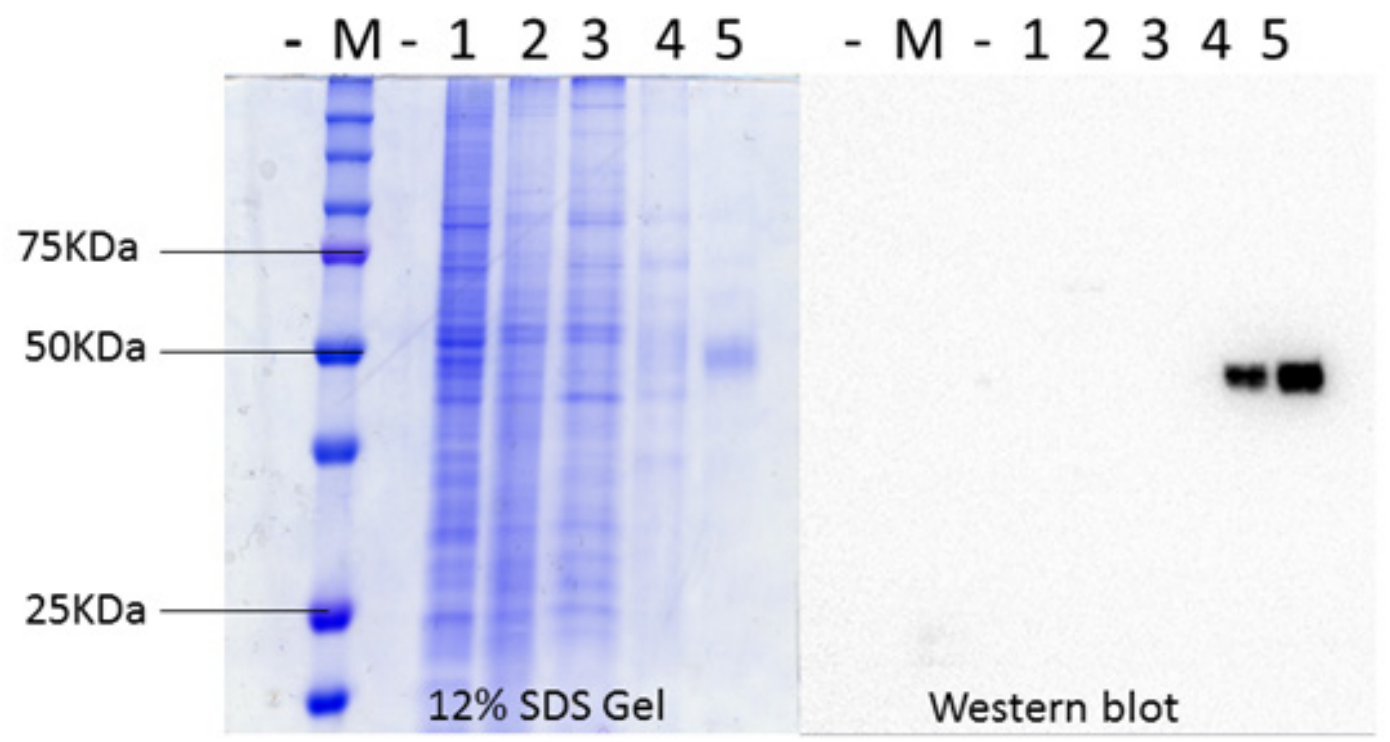

Figure S10: SDS-PAGE and Western blot showing the purification steps of TDP-43 full

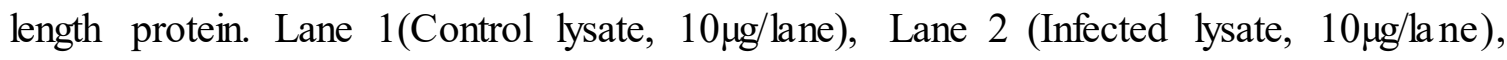

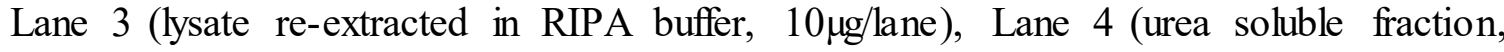
$5 \mu \mathrm{g} / \mathrm{lane}$ ) and Lane 5 (Purified protein, $3 \mu \mathrm{g} / \mathrm{lane}$ ) was loaded on 12\% SDS PAGE and run for 3 hours. For Western blot, all samples were loaded 1/10th of the gel and incubated with Anti-TDP-43 (N-terminal region) produced antibody (1:4000) and Goat anti-rabbit IgG$\operatorname{HRP}(1: 10000)$. 


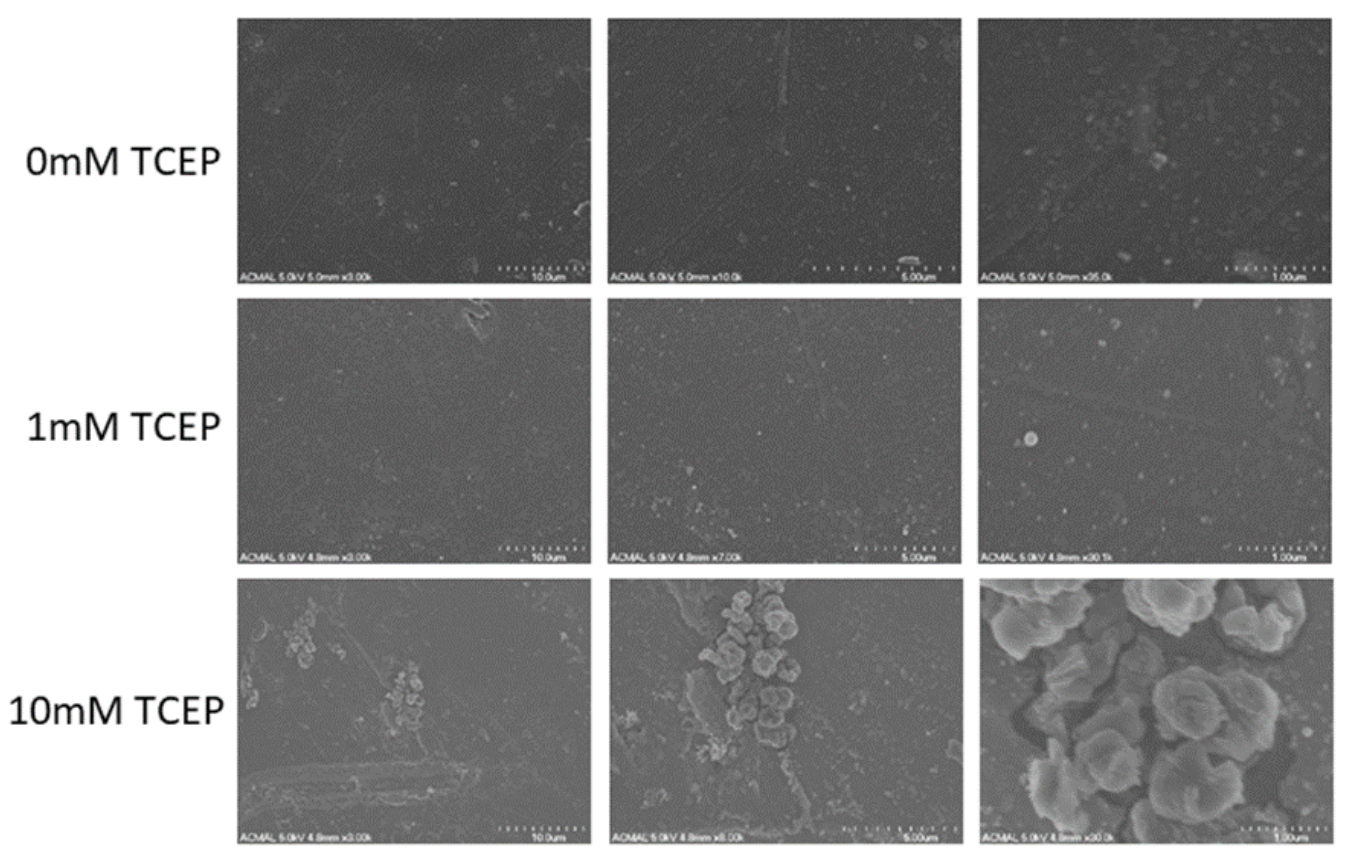

Figure S11: FESEM images of TDP-43 full-length aggregates in presence and absence of TCEP. TDP-43 protein $(1 \mu \mathrm{M})$ was incubated in presence or absence of TCEP $(0,1$ and $10 \mathrm{mM}$ ) for $0 \mathrm{~h}$. Protein aggregates were observed under FESEM. Scale bar $=10 \mu \mathrm{m}, 5 \mu \mathrm{m}$, and $1 \mu \mathrm{m}$ from left to right respectively. 

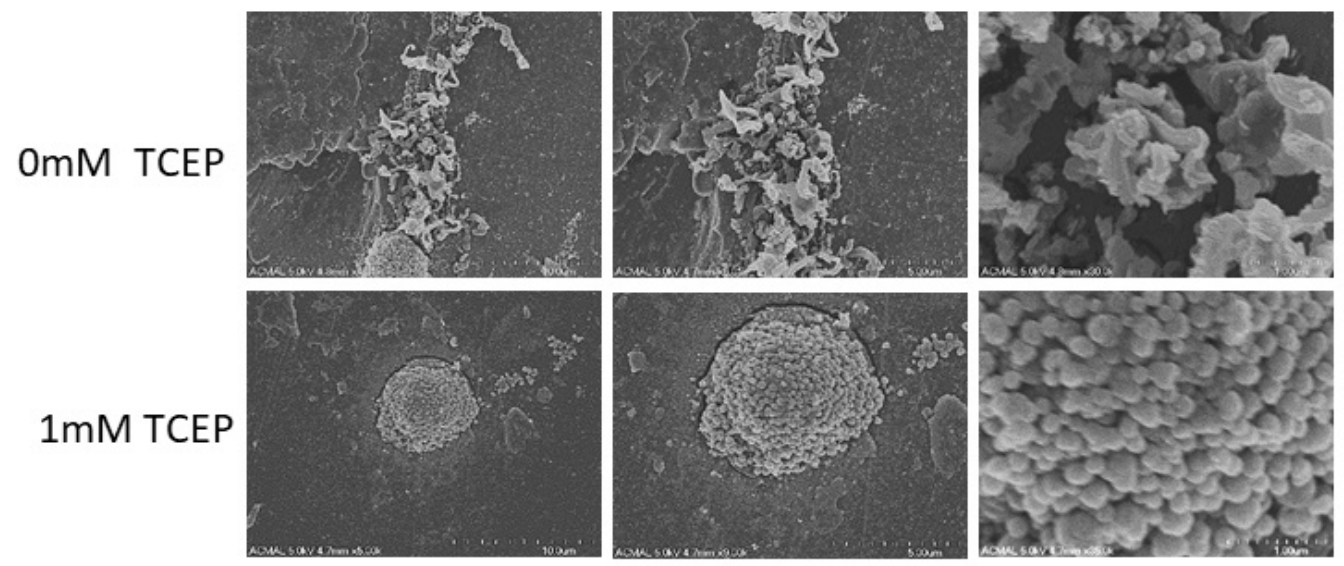

$10 \mathrm{mM}$ TCEP
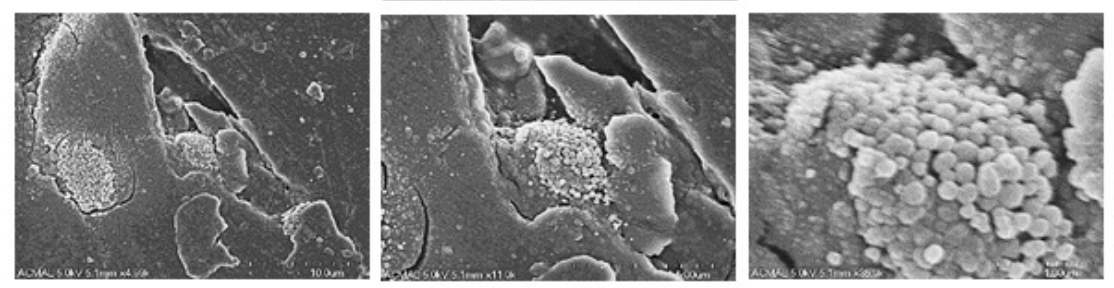

Figure S12: FESEM images of TDP-43 full-length aggregates in presence and absence of TCEP. TDP-43 protein $(1 \mu \mathrm{M})$ was incubated in presence or absence of TCEP $(0,1$ and $10 \mathrm{mM}$ ) for $1 \mathrm{~h}$ at $37^{\circ} \mathrm{C}$. Protein aggregates were observed under FESEM. Scale bar $=10 \mu \mathrm{m}$, $5 \mu \mathrm{m}$, and $1 \mu \mathrm{m}$ from left to right respectively. 


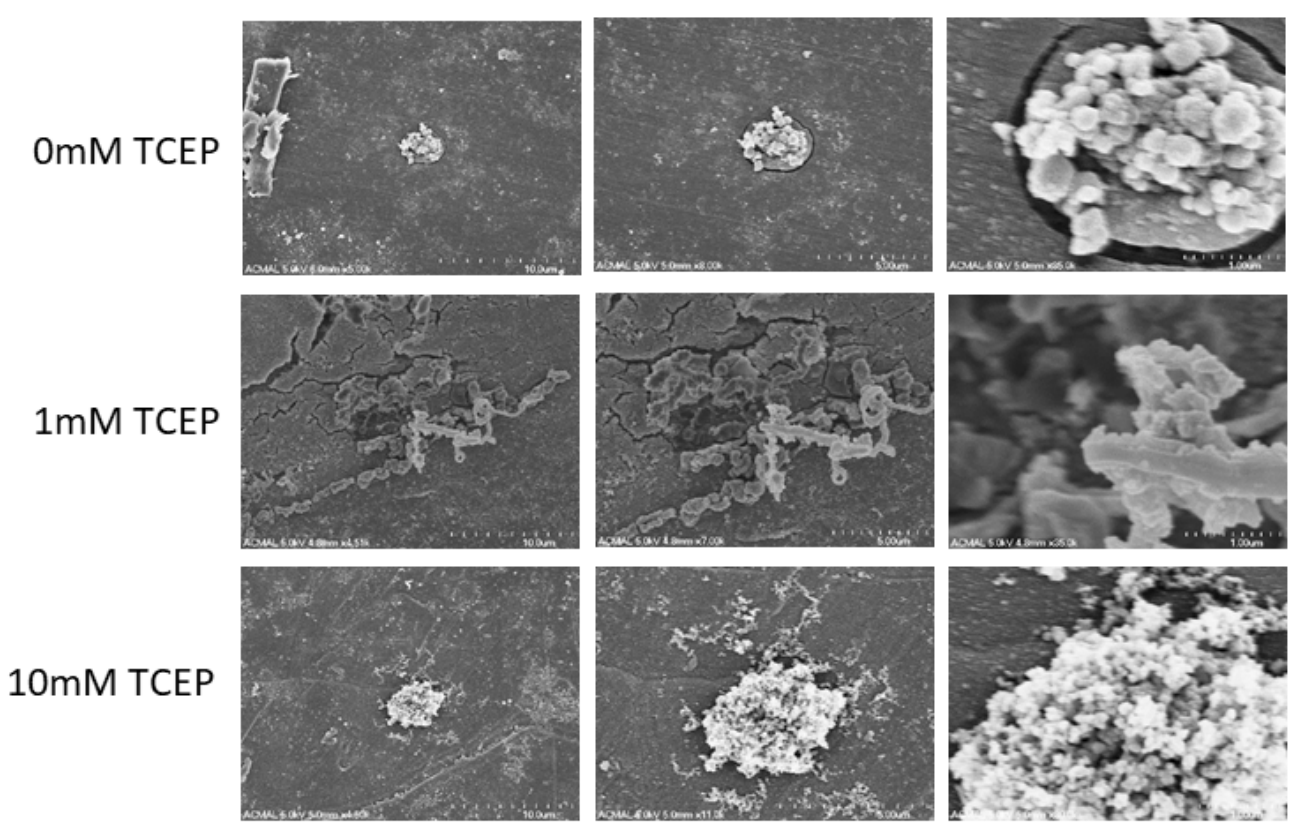

Figure S13: FESEM images of TDP-43 full-length aggregates in presence and absence of TCEP. TDP-43 protein $(1 \mu \mathrm{M})$ was incubated in presence or absence of TCEP $(0,1$ and $10 \mathrm{mM}$ ) for $6 \mathrm{~h}$ at $37^{\circ} \mathrm{C}$. Protein aggregates were observed under FESEM. Scale bar $=10 \mu \mathrm{m}$, $5 \mu \mathrm{m}$, and $1 \mu \mathrm{m}$ from left to right respectively. 

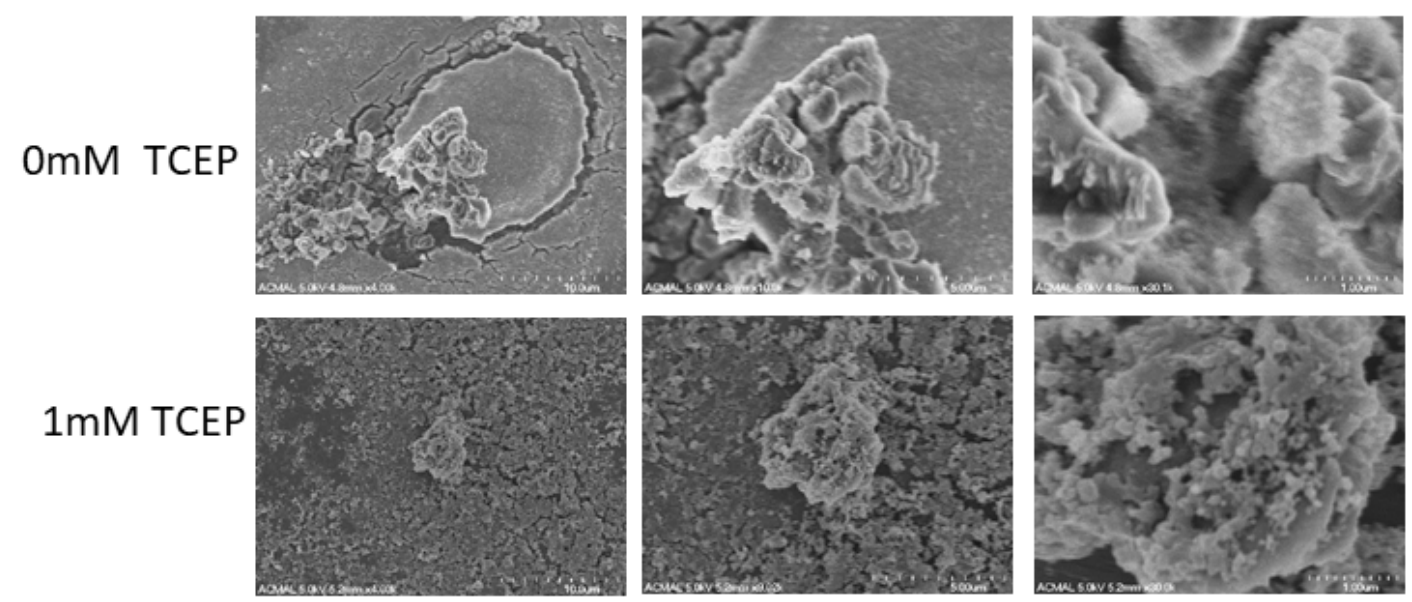

10mM TCEP
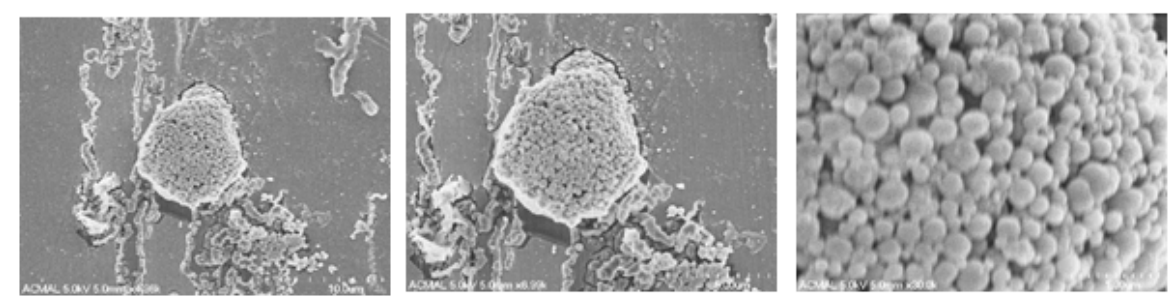

Figure S14: FESEM images of TDP-43 full-length aggregates in presence and absence of TCEP. TDP-43 protein $(1 \mu \mathrm{M})$ was incubated in presence or absence of TCEP $(0,1$ and $10 \mathrm{mM}$ ) for $48 \mathrm{~h}$ at $37^{\circ} \mathrm{C}$. Protein aggregates were observed under FESEM. Scale bar $=10 \mu \mathrm{m}, 5 \mu \mathrm{m}$, and $1 \mu \mathrm{m}$ from left to right respectively. 


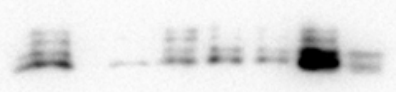

US- urea soluble fraction

BF- Bound flow through

WF- Wash flow through

1-16 - Different Ni-NTA eluted fractions

Primary Antibody: Anti-TDP-43 (C-terminal region) produced antibody (1:4000) Secondary Antibody: Goat anti-rabbit IgG- HRP (1: 10000)

Sample: $20 \mathrm{uL}+10 \mathrm{ul}$ of $3 \mathrm{X}$ sample buffer

Figure S15: Ni-NTA purification of TDP-43 C-terminal construct. Different elution fraction after Ni-NTA purification was run on the $15 \%$ gel and western blot was developed for it using primary and secondary antibody.

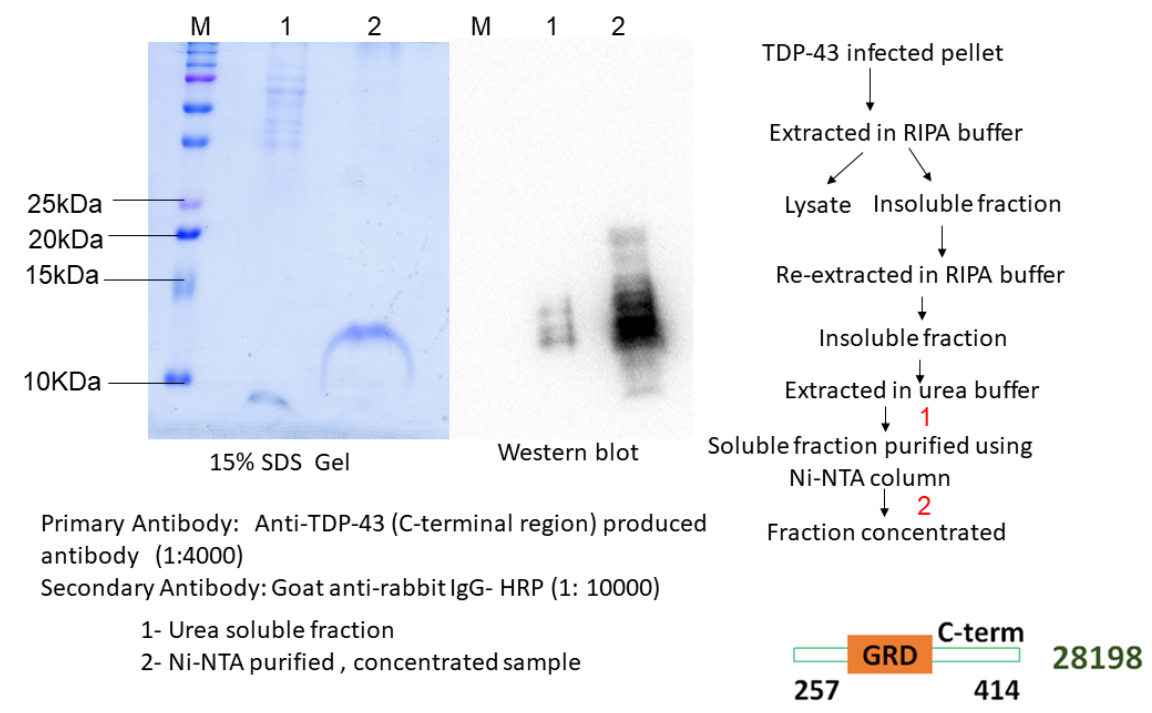


Figure S16: Purification TDP-43 C-terminal construct. TDP-43 construct 28198 containing glycine rich and c-terminal region was subjected to purification as shown in schematic diagram on left and the urea soluble fraction (1) and concentrated Ni-NTA purified sample (2) was loaded on the gel to maximum volume possible. $15 \%$ reducing gel was run for SDS-PAGE and Western blot. For western blot, Anti-TDP-43 (C-terminal region) produced antibody (1:1000) was used as primary antibody and Goat anti-rabbit IgG -HRP (1:10000) was used as secondary antibody. 


\section{Appendix B Supporting Information for chapter 4}

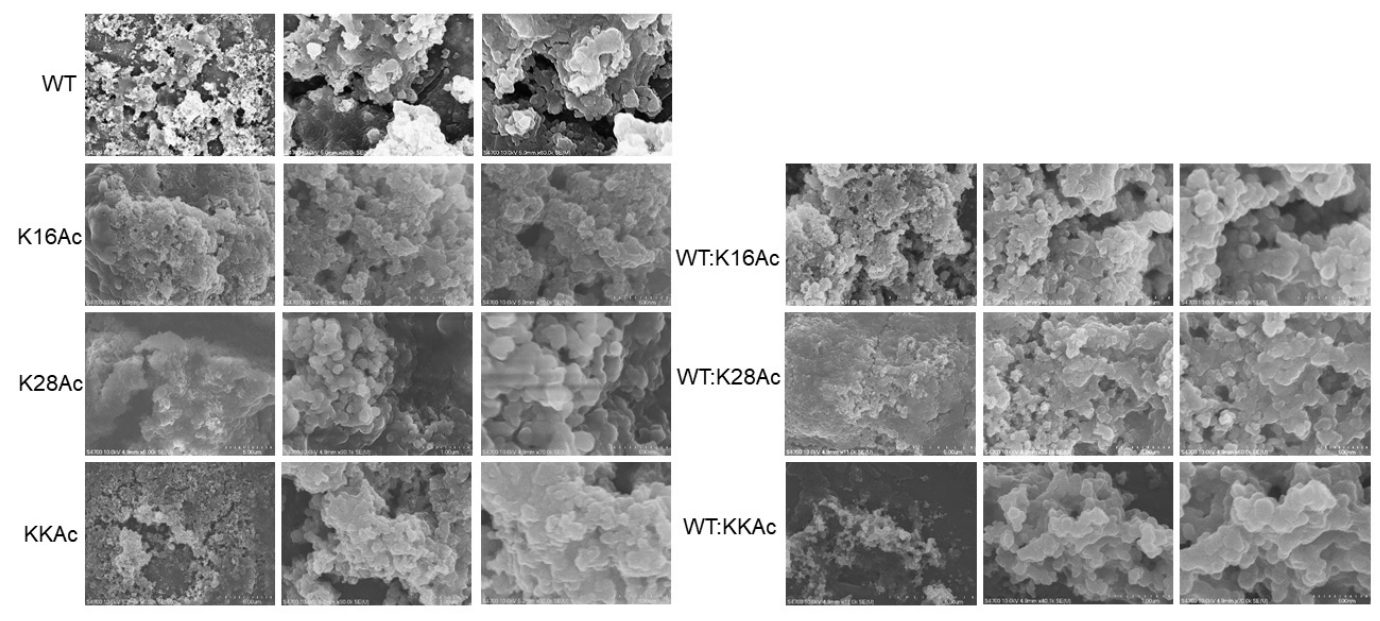

Supplementary Figure 1. SEM images of the WT and acetylated A $\beta 42$ aggregates after

$72 \mathrm{~h}$ of incubation. Scale bars are $5 \mu \mathrm{m}, 1 \mu \mathrm{m}$, and $500 \mathrm{~nm}$ from right to left respectively. 
A WT

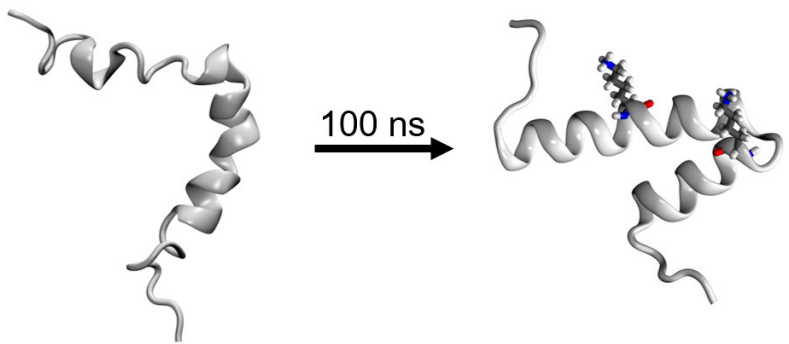

B $\mathrm{K} 16 \mathrm{Ac}$

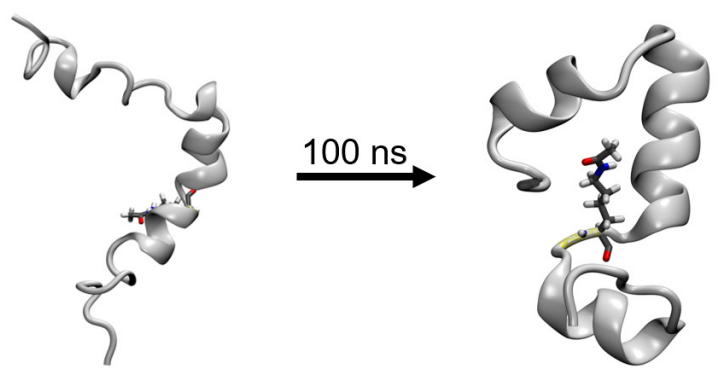

C K28AC
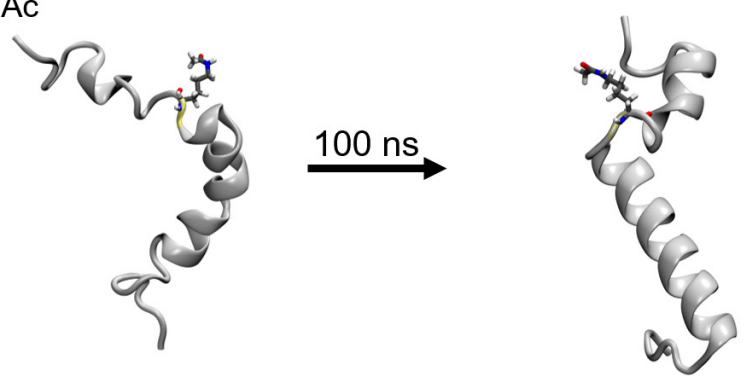

D KKAc
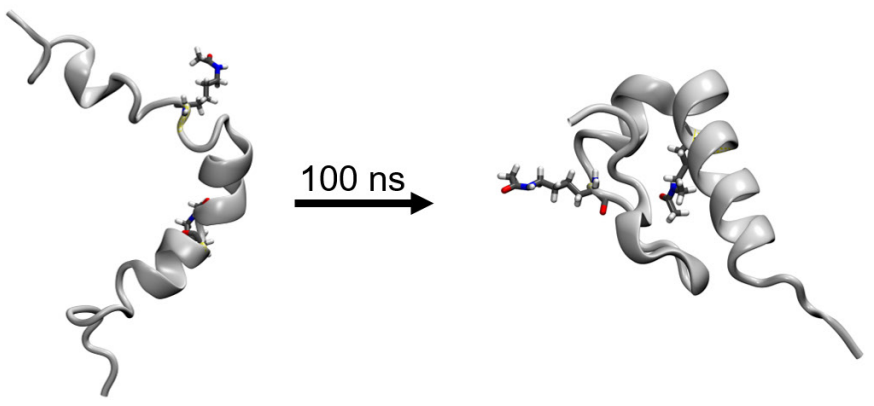

Supplementary Figure 2. Simulation snapshots representing the structural changes in WT and acetylated A $\beta 42$ monomer at $100 \mathrm{~ns}$ in aqueous solution. (A) WT, (B) K16Ac, (C) K28Ac and (D) KKAc. The waters molecules are not shown for clarity. The inset figure highlights the $\alpha$-helix, $\beta$-turn, and random coil regions in WT A $\beta 42$ monomer. 
A WT

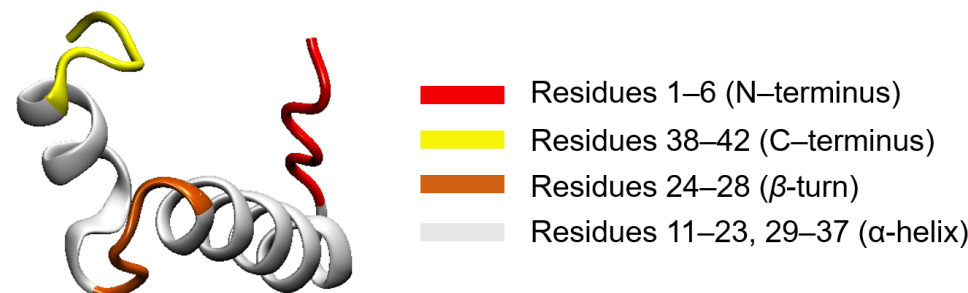

B K16Ac

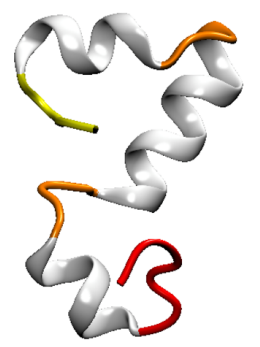

Residues 1-6 (N-terminus)

Residues 38-42 (C-terminus)

Residues 29-31, 14-17 ( $\beta$-turn)

Residues 11-13, 18-28, 32-37 ( $\alpha$-helix)

C $\mathrm{K} 28 \mathrm{Ac}$

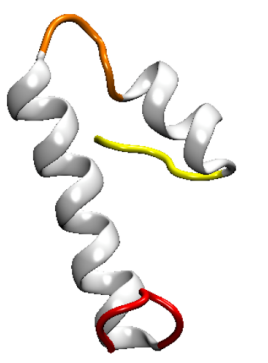

Residues 1-6 (N-terminus)

Residues 38-42 (C-terminus)

Residues 26-30 ( $\beta$-turn)

Residues 11-23, 31-37 ( $\alpha$-helix)

D KKAc

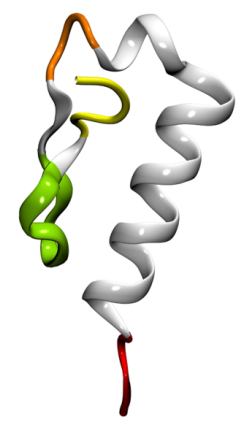

Residues 1-6 (N-terminus)

Residues 38-42 (C-terminus)

Residues 26-29 ( $\beta$-turn)

Residues 11-19 ( $\alpha$-helix)

Residues 31-37 (pleat)

Supplementary Figure 3. The $\alpha$-helix, $\beta$-turn, and random coil regions in A $\beta 42$ monomer. (A) WT, (B) K16Ac, (C) K28Ac and (D) KKAc. 


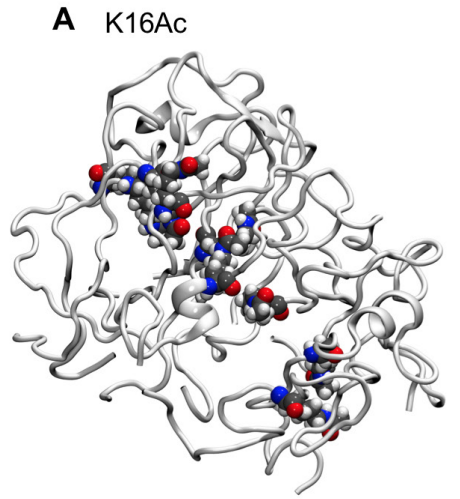

B K28Ac

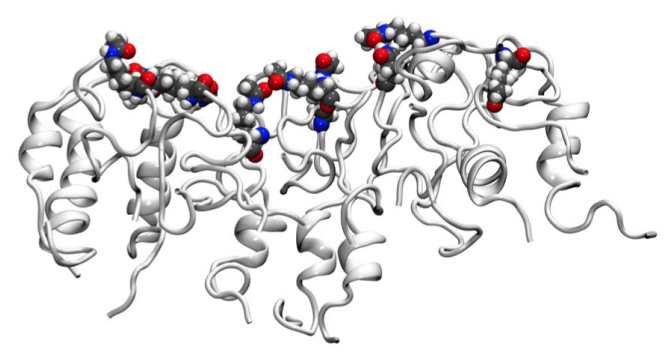

Supplementary Figure 4. Orientation of (A) K16Ac residues in 9 strands of K16Ac peptides, and (B) K28 residues in 9 strands of K28Ac aggregates at $100 \mathrm{~ns}$. 

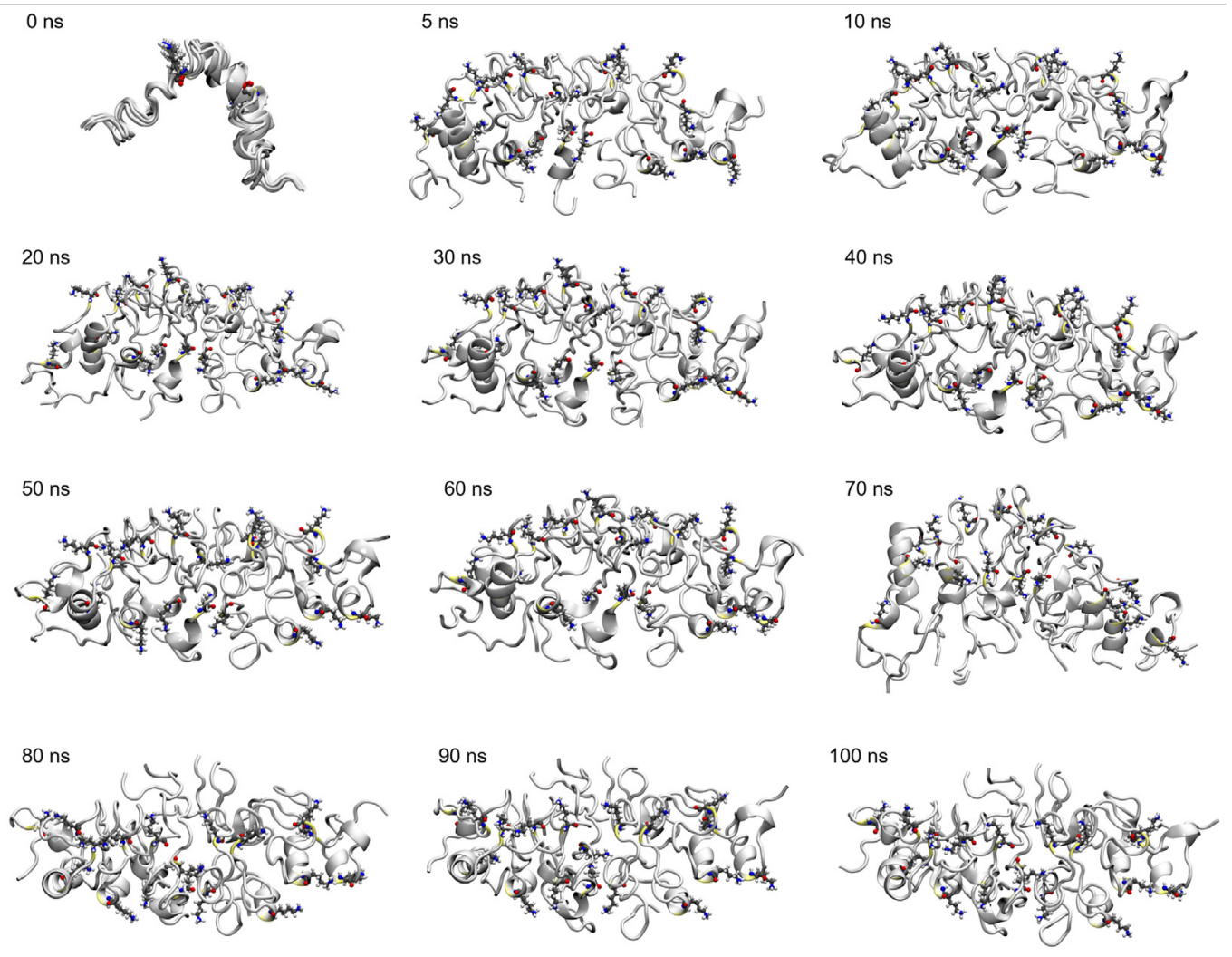

Supplementary Figure 5. Simulation snapshots depicting the structural changes in aggregation of 9 strands of WT A $\beta 42$ peptides for 100 ns of dynamics. The waters molecules are not shown for clarity. 

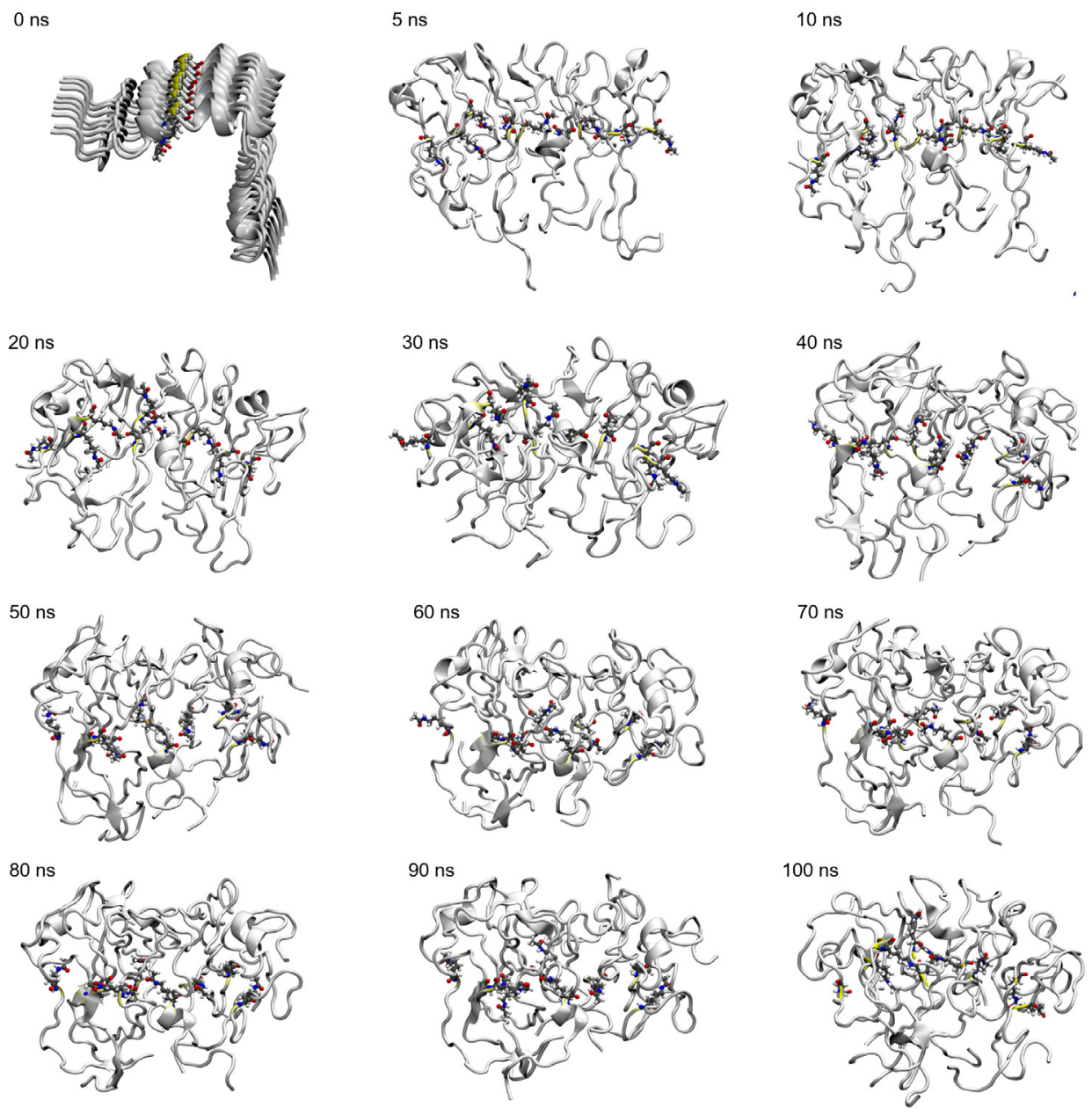

Supplementary Figure 6. Simulation snapshots depicting the structural changes in aggregation of 9 strands of K16Ac peptides for $100 \mathrm{~ns}$ of dynamics. The waters molecules are not shown for clarity. 


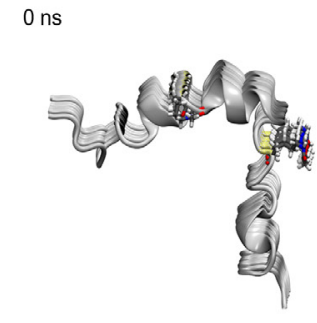

$20 \mathrm{~ns}$
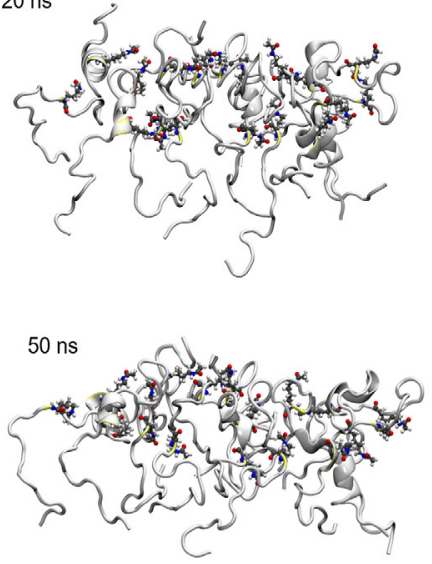

80 ns

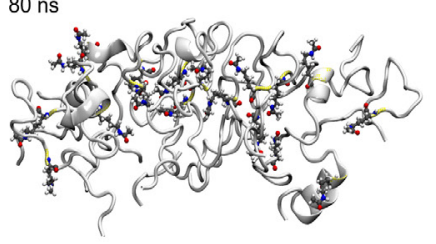

$5 \mathrm{~ns}$

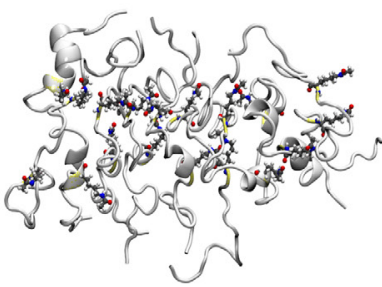

30 ns
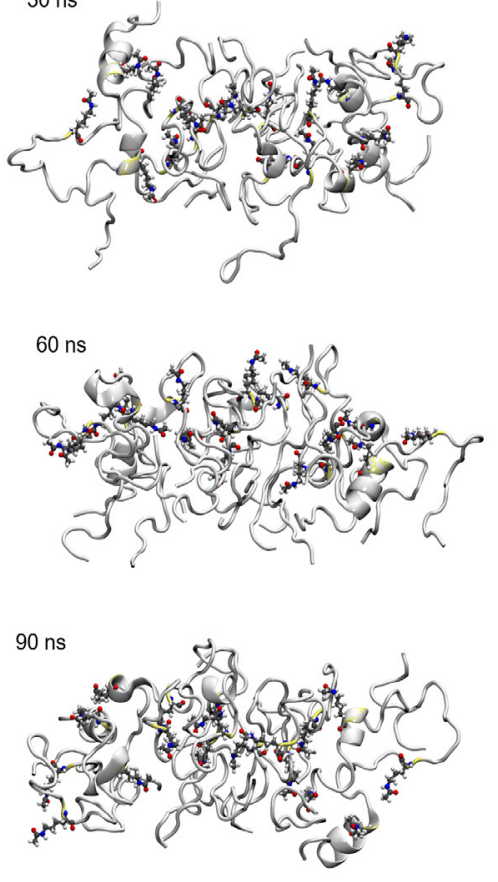

$10 \mathrm{~ns}$

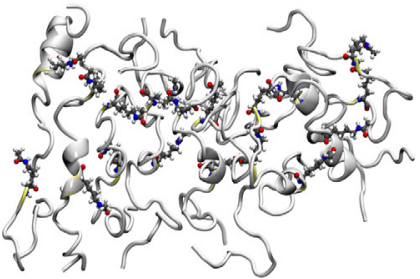

$40 \mathrm{~ns}$
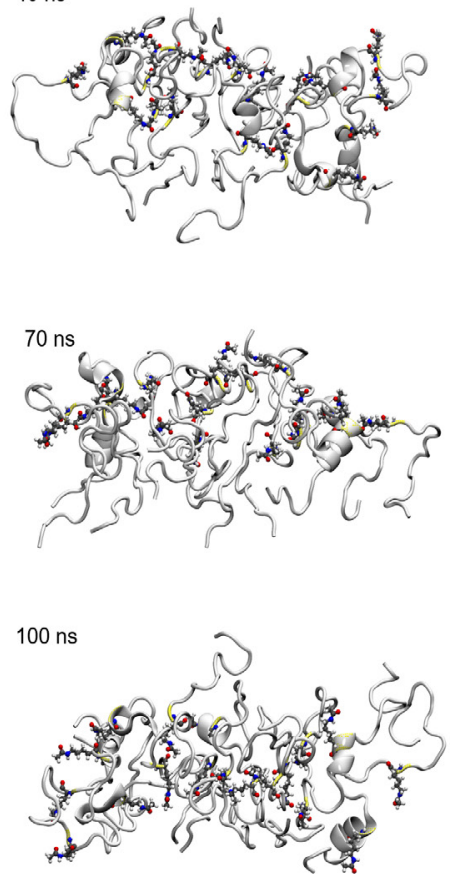

Supplementary Figure 7. Simulation snapshots depicting the structural changes in aggregation of 9 strands of KKAc peptides for $100 \mathrm{~ns}$ of dynamics. The waters molecules are not shown for clarity. 

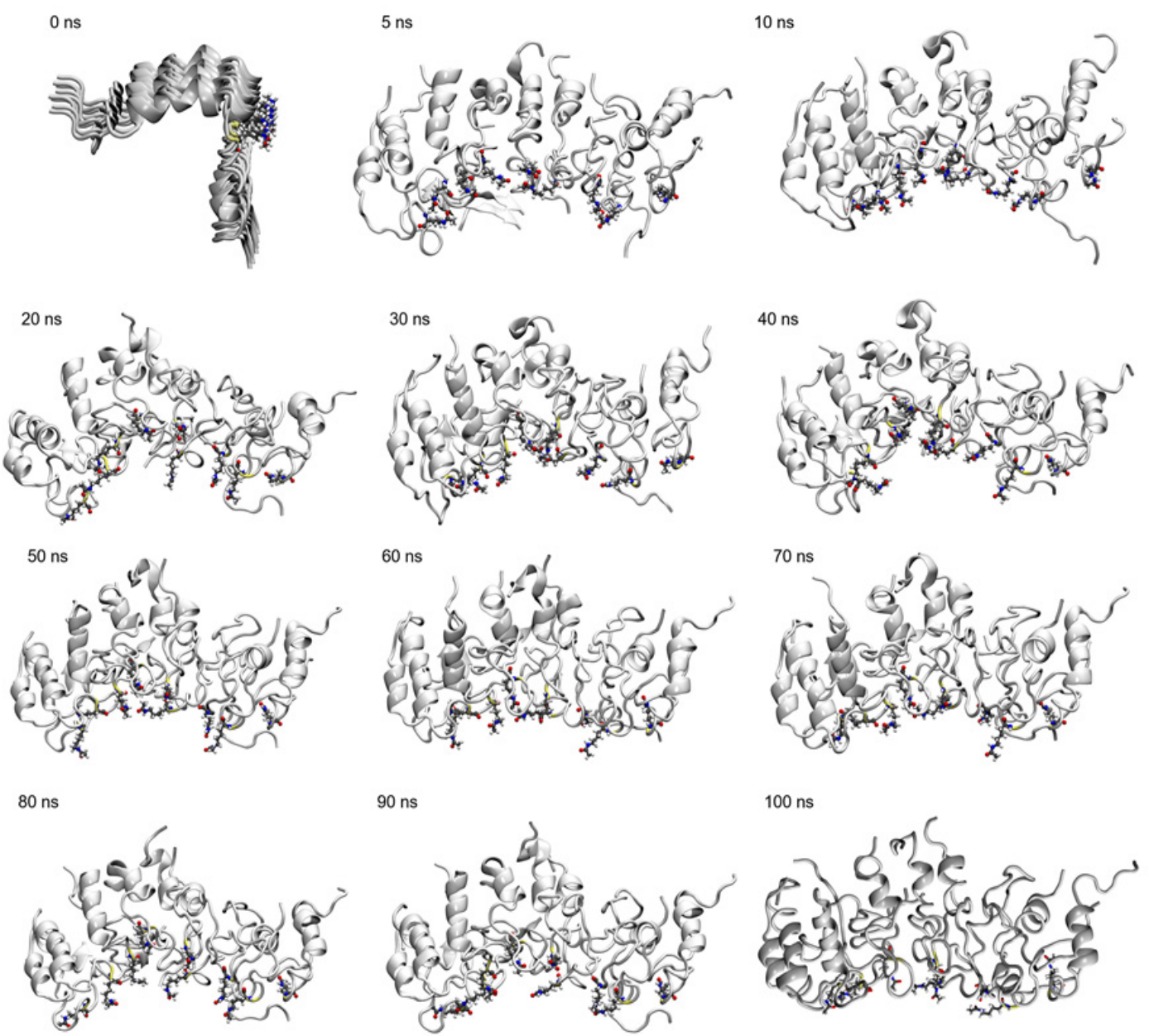

Supplementary Figure 8. Simulation snapshots depicting the structural changes in aggregation of 9 strands of K28Ac peptides for $100 \mathrm{~ns}$ of dynamics. The waters molecules are not shown for clarity. 


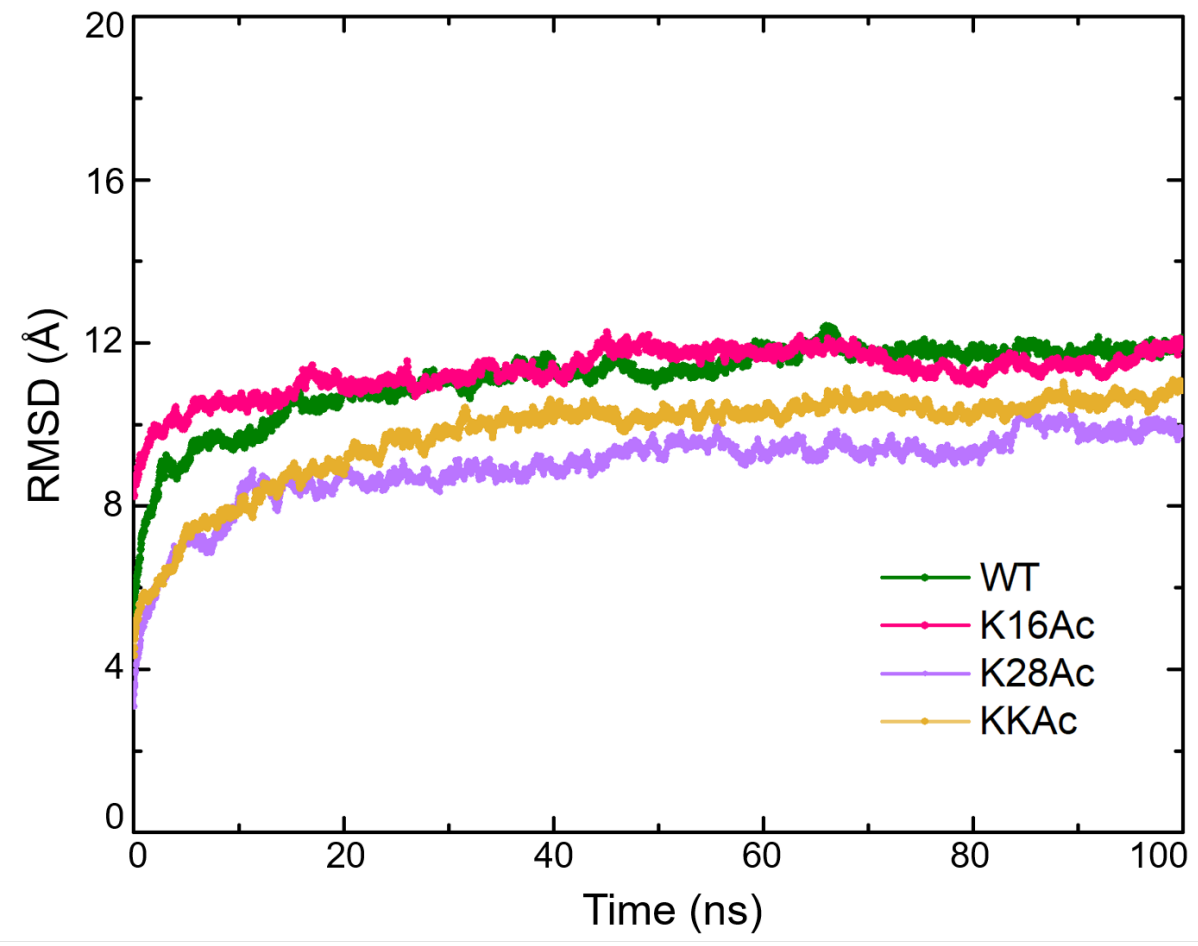

Supplementary Figure 9. (A) Root mean-square deviation (RMSD) in 9 strands of WT, $\mathrm{K} 16 \mathrm{Ac}, \mathrm{K} 28 \mathrm{Ac}$, and KKAc A $\beta 42$ peptides in aqueous solution. 


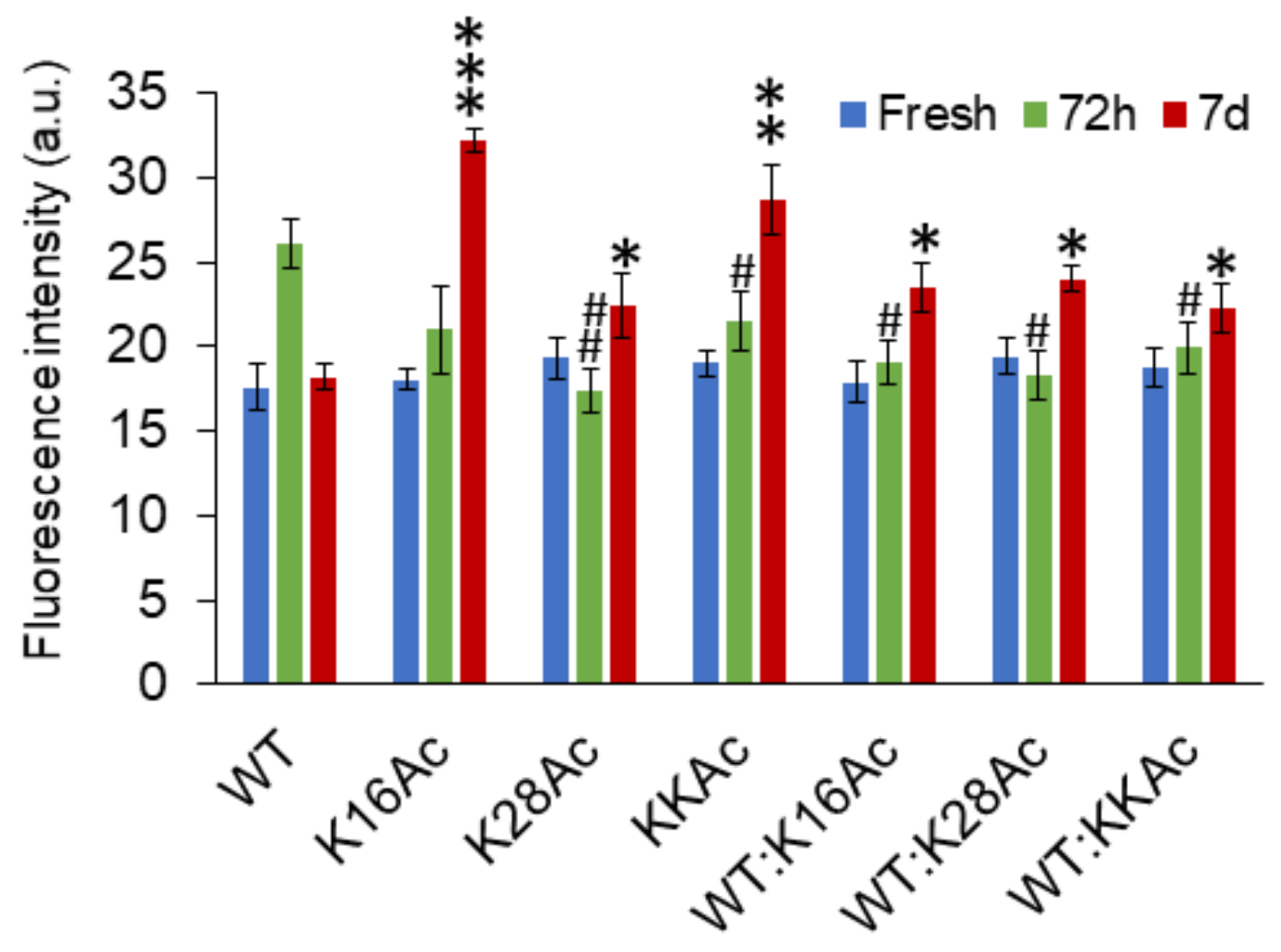

Supplementary Figure 10. Hydrophobicity of fresh A $\beta 42$ peptides and their aggregates measured by ANS fluorescence by incubating $10 \mu \mathrm{M}$ of peptide samples with $10 \mu \mathrm{M}$ of ANS and measuring peak intensities at $485 \mathrm{~nm}$. Mixture represents the 1:1 ratio of the acetylated peptides with WT A $\beta 42$ peptides. Error bars $= \pm$ S.D. $(\mathrm{n}=3)$. *, **, and *** represents significant differences $(*=p<0.05),(* *=p<0.005)$, and $(* * *=p<0.0005)$. $(+, \#, *$ represents statistics for Fresh, $72 \mathrm{~h}$ and $7 \mathrm{~d}$ data respectively and significant differences are set identical for all time points).

Note: Statistical analysis (one-way ANOVA) for $7 \mathrm{~d}$ sample is included at the end of the document. For Fresh and $72 \mathrm{~h}$ sample statistical analysis as separate file is included. 


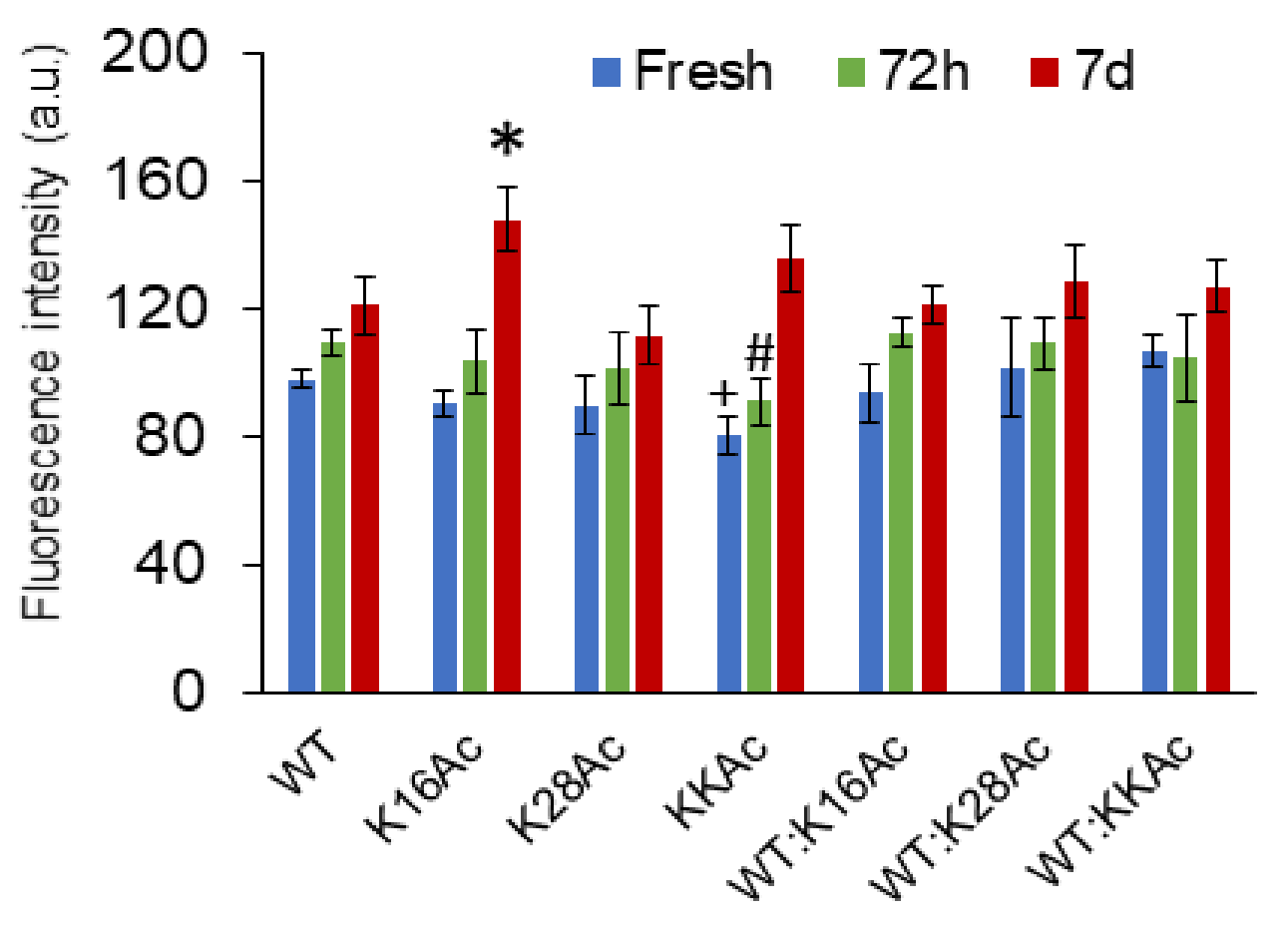

Supplementary Figure 11. Structure flexibility of the fibrils or aggregates was measured by bis-ANS fluorescence by incubating $10 \mu \mathrm{M}$ of peptide samples with $1 \mu \mathrm{M}$ of bis-ANS and measuring peak intensities at $484 \mathrm{~nm}$. Error bars $= \pm$ S.D. $(\mathrm{n}=3)$. +,\#,* represents statistics for Fresh, $72 \mathrm{~h}$ and $7 \mathrm{~d}$ data respectively and significant difference are set identical for all time points.,$+{ }^{*}$ represents significant differences $(p<0.05)$.

Note: Statistical analysis (one-way ANOVA) for $7 \mathrm{~d}$ sample is included at the end of the document. For Fresh and $72 \mathrm{~h}$ sample statistical analysis as separate file is included. 

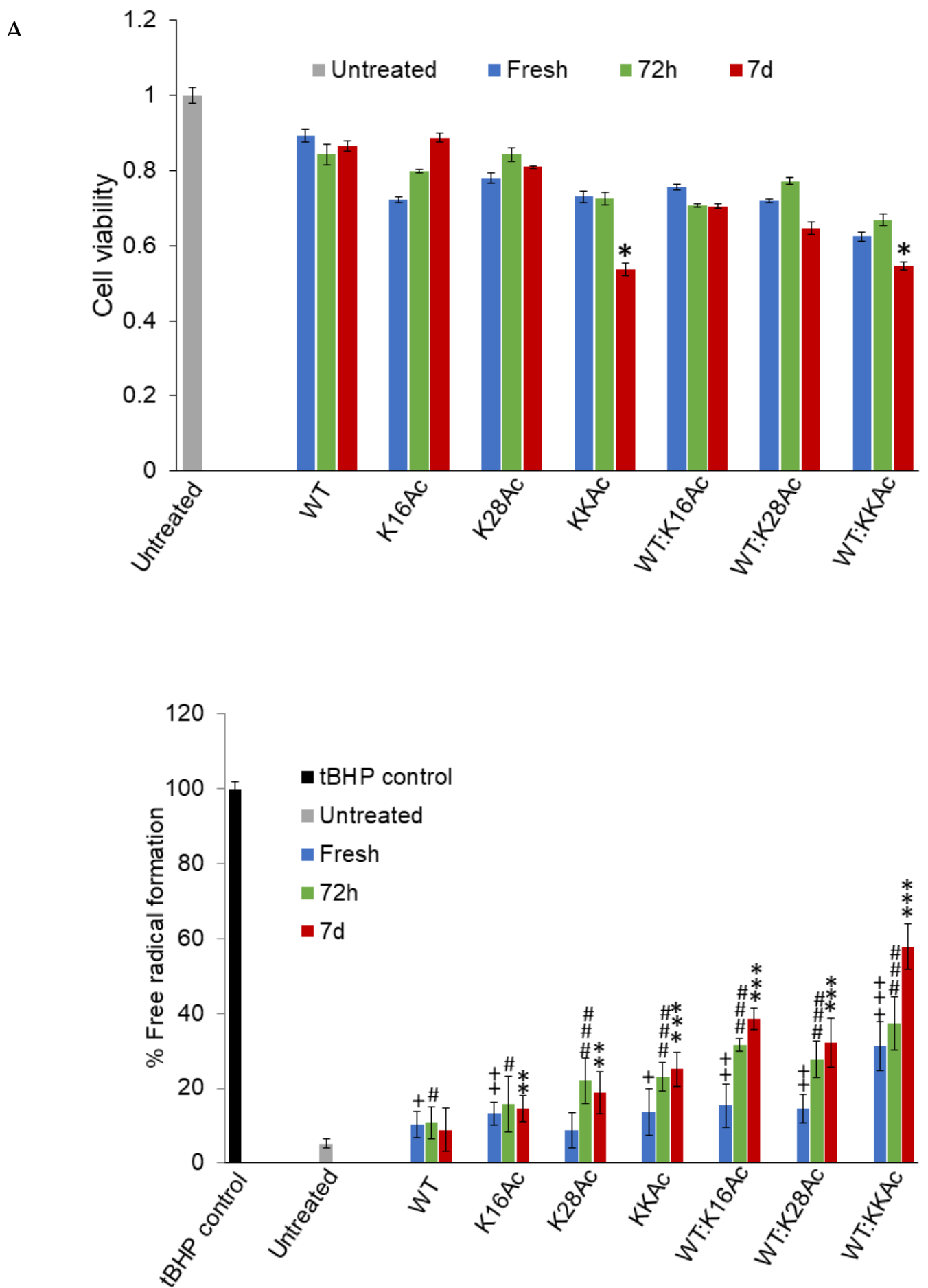

The effects of $2 \mu \mathrm{M}$ of WT and acetylated fresh A $\beta 42$ peptides and their aggregates on SH-SY5Y neuroblastoma cells health measured by MTS and DCF-HDA assay. Refer next page for more details. 


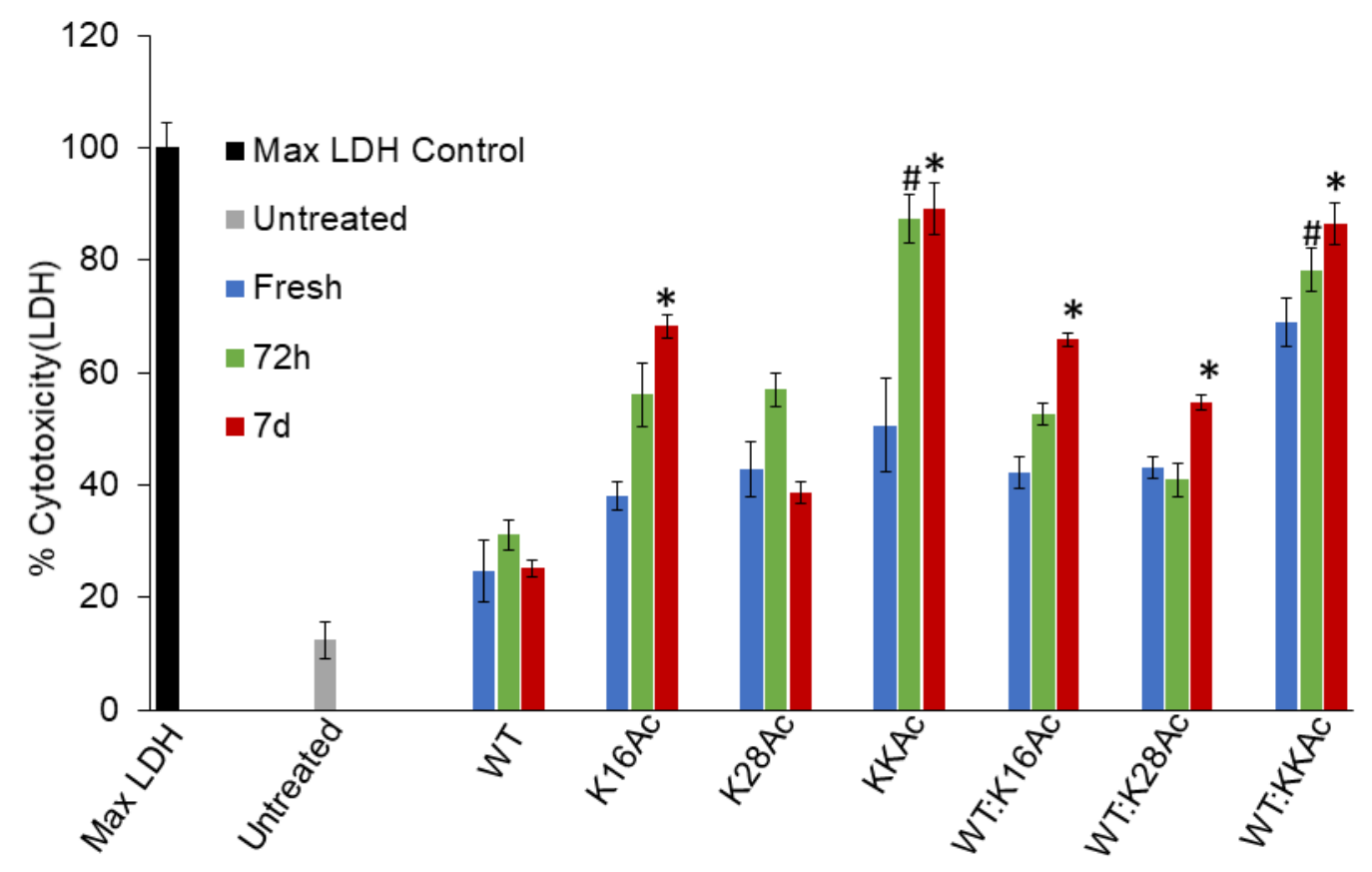

Supplementary Figure 12. The effect of $2 \mu \mathrm{M}$ of WT and acetylated fresh $\mathrm{A} \beta 42$ peptides and their aggregates on SH-SY5Y neuroblastoma cells health were measured by (A) MTS assay, (B) DCFH-DA assay, and (C) LDH assay. The fresh peptides, $72 \mathrm{~h}$, or $7 \mathrm{~d}$ aggregates were added to the cells to a final concentration of $2 \mu \mathrm{M}$. Cells were incubated for $48 \mathrm{~h}$ in presence of peptides for MTS and LDH assay and for $24 \mathrm{~h}$ for DCFH-DA assay. For tbutyl-hydroperoxide (tBHP) control for DCFH-DA assay, cells were incubated with $50 \mu \mathrm{M}$ of tBHP for $4 \mathrm{~h}$ at $37^{\circ} \mathrm{C}$. The mixtures represent samples containing equimolar (1:1) WT and acetylated peptides. Error bars $= \pm$ S.D. (for $\mathrm{A} n=6$; for $\mathrm{B} \mathrm{n}=5$; and for $\mathrm{C} \mathrm{n}=6$ ). *, $* *$, and $* * *$ represents significant differences $(*=p<0.05),(* *=p<0.005)$, and $(* * *=$ $p<0.0005) .(+, \#, *$ represents statistics for Fresh, $72 \mathrm{~h}$ and $7 \mathrm{~d}$ data respectively and significant differences are set identical for all time points).

Note: Statistical analysis (one-way ANOVA) for $7 \mathrm{~d}$ sample is included at the end of the document. For Fresh and $72 \mathrm{~h}$ sample statistical analysis as separate file is included. 

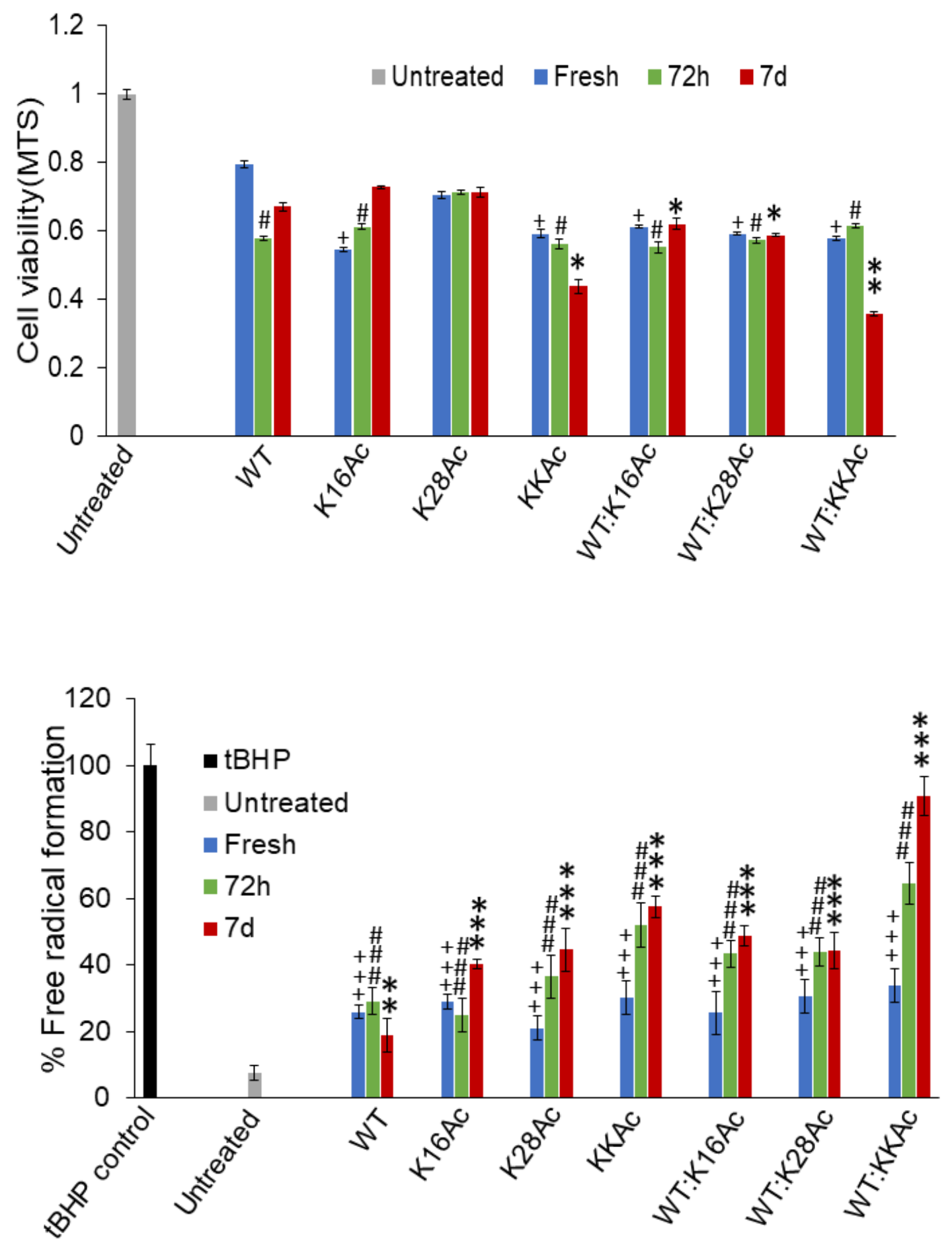

The effects of $5 \mu \mathrm{M}$ of WT and acetylated fresh A $\beta 42$ peptides and their aggregates on SH-SY5Y neuroblastoma cells health measured by MTS and DCF-HDA assay. Refer next page for more details. 


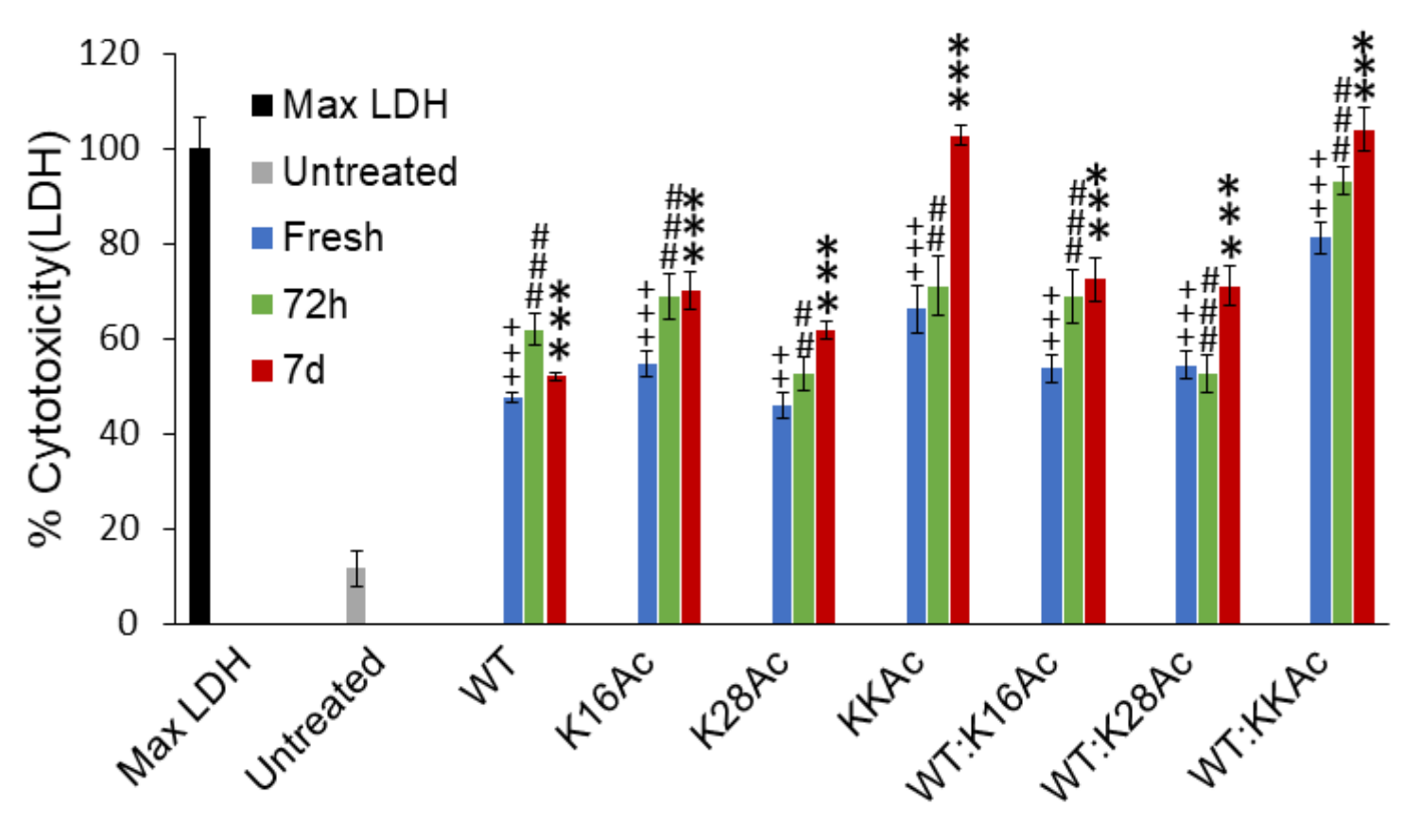

Supplementary Figure 13. The effect of $5 \mu \mathrm{M}$ of WT and acetylated fresh A $\beta 42$ peptides and their aggregates on SH-SY5Y neuroblastoma cells health were measured by (A) MTS assay, (B) DCFH-DA assay, and (C) LDH assay. The fresh peptides, $72 \mathrm{~h}$, or $7 \mathrm{~d}$ aggregates were added to the cells to a final concentration of $5 \mu \mathrm{M}$. Cells were incubated for $48 \mathrm{~h}$ in presence of peptides for MTS and LDH assay and for $24 \mathrm{~h}$ for DCFH-DA assay. For tbutyl-hydroperoxide (tBHP) control for DCFH-DA assay, cells were incubated with $50 \mu \mathrm{M}$ of tBHP for $4 \mathrm{~h}$ at $37^{\circ} \mathrm{C}$. The mixtures represent samples containing equimolar (1:1) WT and acetylated peptides. Error bars $= \pm$ S.D. (for $\mathrm{A} n=6$; for $\mathrm{B} \mathrm{n}=5$; and for $\mathrm{C} \mathrm{n}=5$ ). *, $* *$, and $* * *$ represents significant differences $(*=p<0.05),(* *=p<0.005)$, and $(* * *=$ $p<0.0005)$. (+,\#,* represents statistics for Fresh, $72 \mathrm{~h}$ and $7 \mathrm{~d}$ data respectively and significant difference are set identical for all time points).

Note: Statistical analysis (one-way ANOVA) for $7 \mathrm{~d}$ sample is included at the end of the document. For Fresh and $72 \mathrm{~h}$ sample statistical analysis as separate file is included. 

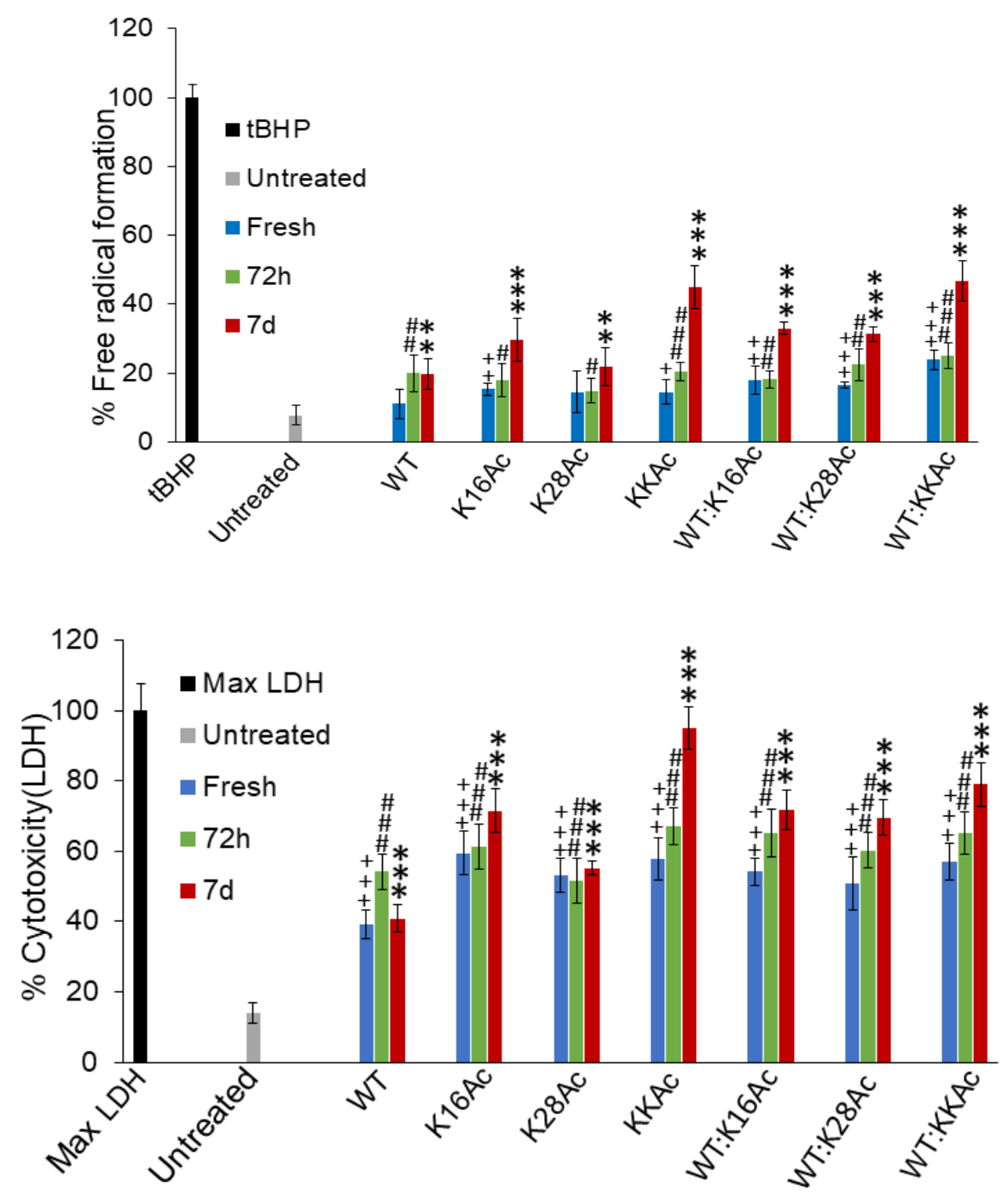

Supplementary Figure 14. The effect of $2 \mu \mathrm{M}$ of WT and acetylated fresh A $\beta 42$ peptides and their aggregates on primary neuronal cells health were measured by (A) DCFH-DA assay, and (B) LDH assay. The fresh peptides, $72 \mathrm{~h}$, or $7 \mathrm{~d}$ aggregates were added to the cells to a final concentration of $2 \mu \mathrm{M}$. Cells were incubated for $24 \mathrm{~h}$ in presence of peptides for both assays. For t-butyl-hydroperoxide (tBHP) control for DCFH-DA assay, cells were incubated with $50 \mu \mathrm{M}$ of tBHP for $4 \mathrm{~h}$ at $37^{\circ} \mathrm{C}$. The mixtures represent samples containing equimolar (1:1) WT and acetylated peptides. Error bars $= \pm$ S.D. (for An $=5$; for B $n=4$ ). $*$, **, and $* * *$ represents significant differences $(*=p<0.05),(* *=p<0.005)$, and $(* * *$ $=p<0.0005) .(+, \#, *$ represents statistics for Fresh, $72 \mathrm{~h}$ and $7 \mathrm{~d}$ data respectively and significant difference are set identical for all time points).

Note: Statistical analysis (one-way ANOVA) for $7 \mathrm{~d}$ sample is included at the end of the document. 


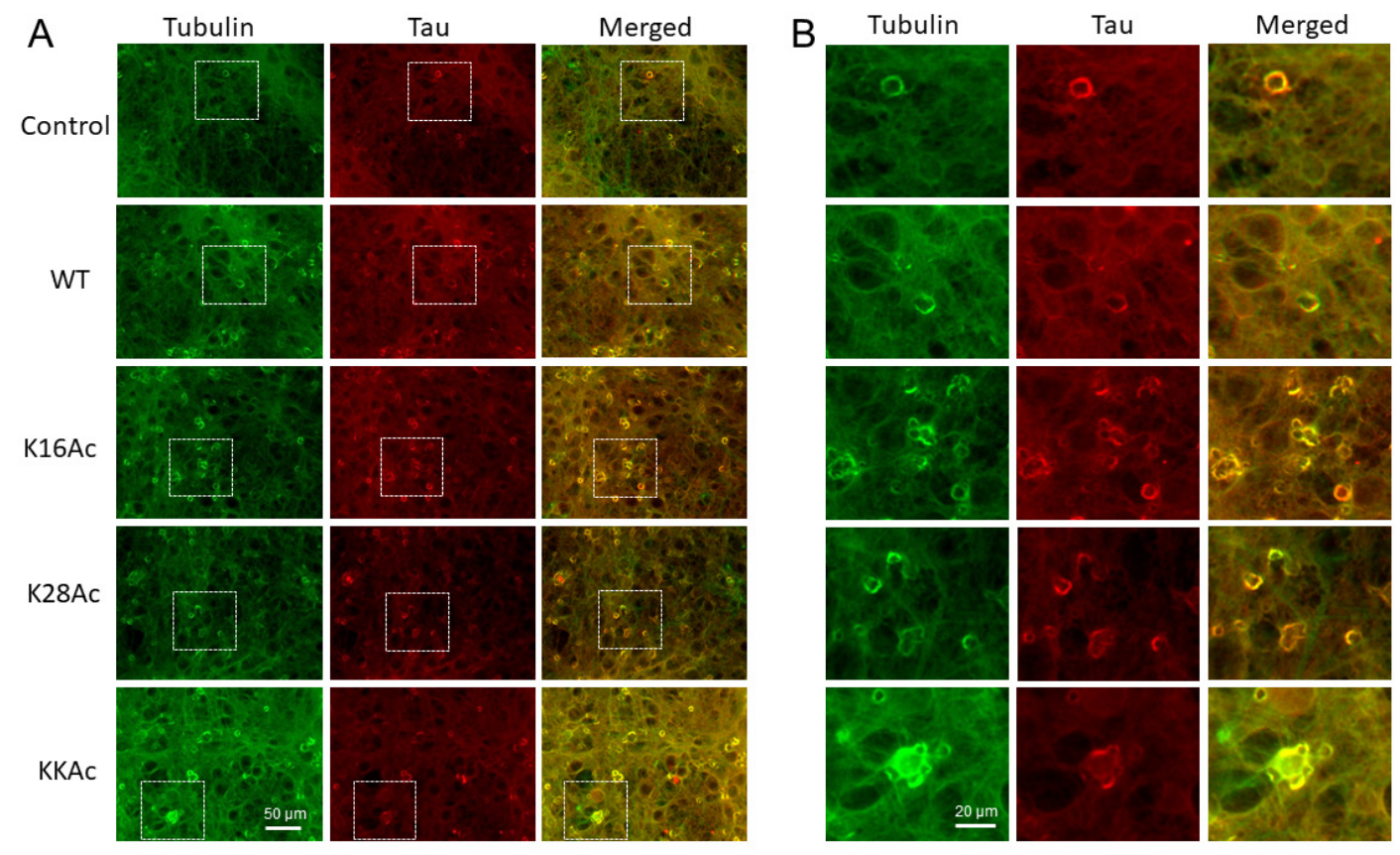

Supplementary Figure 15. Immunostaining of primary neuronal cells from rat brain. Primary neuronal cells were incubated with $2 \mu \mathrm{M}$ of $7 \mathrm{~d}$ incubated A $\beta 42$ WT and acetylated peptides for $24 \mathrm{~h}$ at $37{ }^{\circ} \mathrm{C}$. Control indicates cells that are untreated and incubated under identical conditions with which A $\beta 42$ treated cells were compared. The cells were fixed and then stained for immunofluorescence with antibodies for tubulin (green) and Tau (red). The images were acquired using Leica DMIL LED at 20X magnification. Scale bar $=50$ $\mu \mathrm{m}$ (A). Zoomed in images of (A) are represented in (B) and are shown in boxes in panel A. Scale bar for panel $B=20 \mu \mathrm{m}$. 


\section{Silver staining of A $\beta$ WT and acetylated peptides}

For silver staining 15\% homemade SDS-PAGE was used and followed the manufacturer's instructions (ThermoScientific). In brief, the $A \beta 7 \mathrm{~d}$ incubated samples were mixed with $3 \mathrm{X}$ sample loading buffer and boiled for 3-5 minutes. All samples were loaded at the final concentration of $2 \mu \mathrm{g} / \mathrm{lane}$ and ran for 3 hours at $80 \mathrm{~V}$. After the run, the gel was rinsed with MilliQ water for 5 minutes with two repeats. All steps were carried out in shaker with gentle agitation. The gel was fixed with fixing solution (30\% Ethanol: $10 \%$ acetic acid) for 15 minutes and repeated for another 15 minutes followed by two washes in 10\% Ethanol at the interval of 5 minutes. Next, the gel was washed twice in MilliQ water for 5 minutes and sensitized for 1 minutes with Silver stain sensitizer working solution. The gel was then washed twice with MilliQ water for 1 minutes and incubated in Silver stain working solution for 30 minutes. After the incubation, the gel was washed twice for 20 seconds and the gel was developed by adding the Developer working solution. Once the desired band intensity were observed the stop solution (5\% Acetic acid) was added. The gel was further incubated for 10 minutes in stop solution and the image was captured using Epson scanner. The gel was quantified using the Image $J$ software and the plot was plotted for the ratio of High molecular weight vs monomer. 

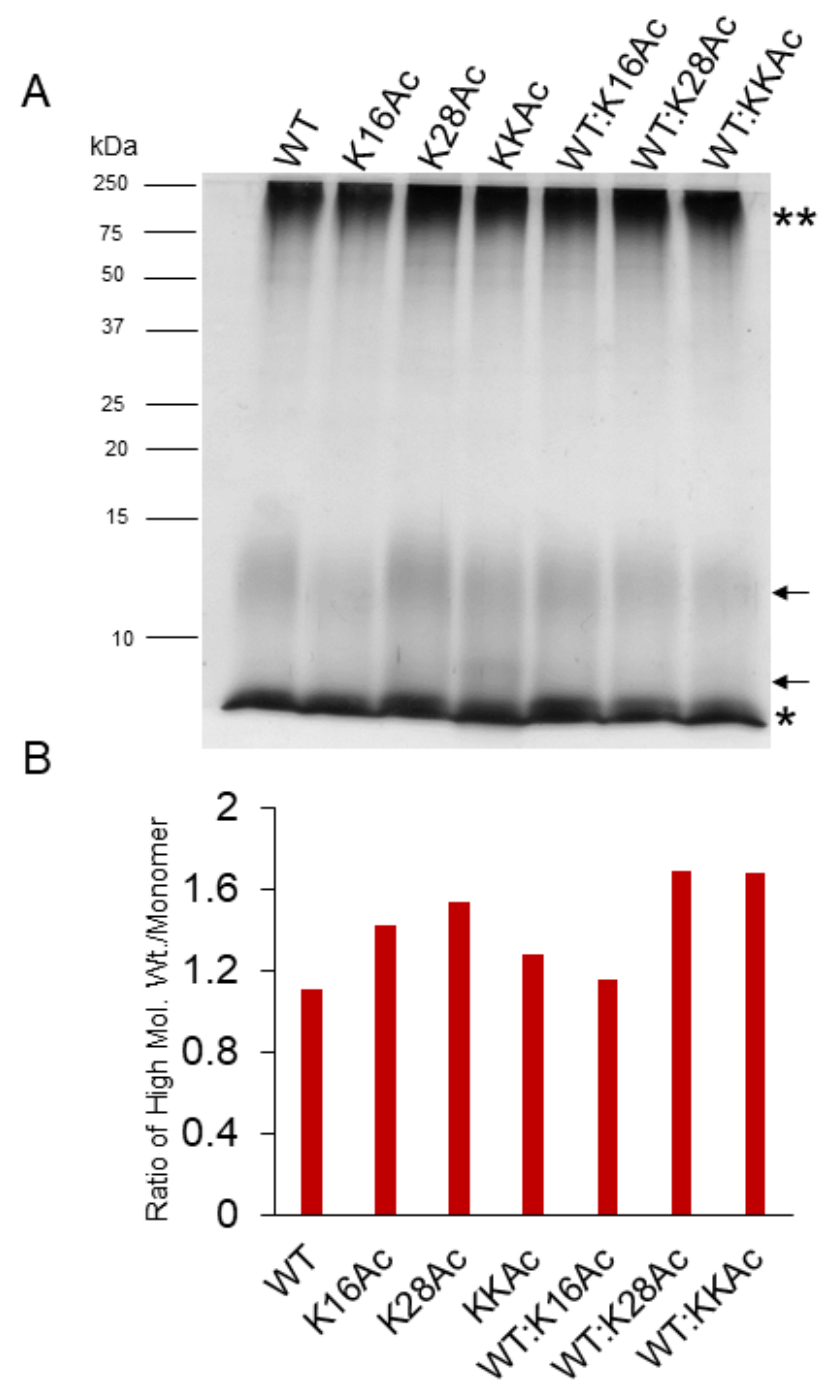

Supplementary Figure 16. Silver staining of $7 \mathrm{~d}$ incubated WT, acetylated, and mixture of A $\beta 42$ peptides. Samples were mixed with $3 \mathrm{X}$ sample loading buffer and boiled for 3-5 minutes. All samples were loaded $2 \mu \mathrm{g} /$ lane in $15 \%$ gel and ran for 3 hours at $80 \mathrm{~V}$. The gel was then subjected to silver staining as per manufacturer's instructions. * and ** represents monomer and high molecular weight respectively. $(\leftarrow)$ represents the oligomeric species of varying molecular weight (A). The gel was quantified using the Image J software and the data was plotted as ratio of high molecular weight vs monomer (B). 


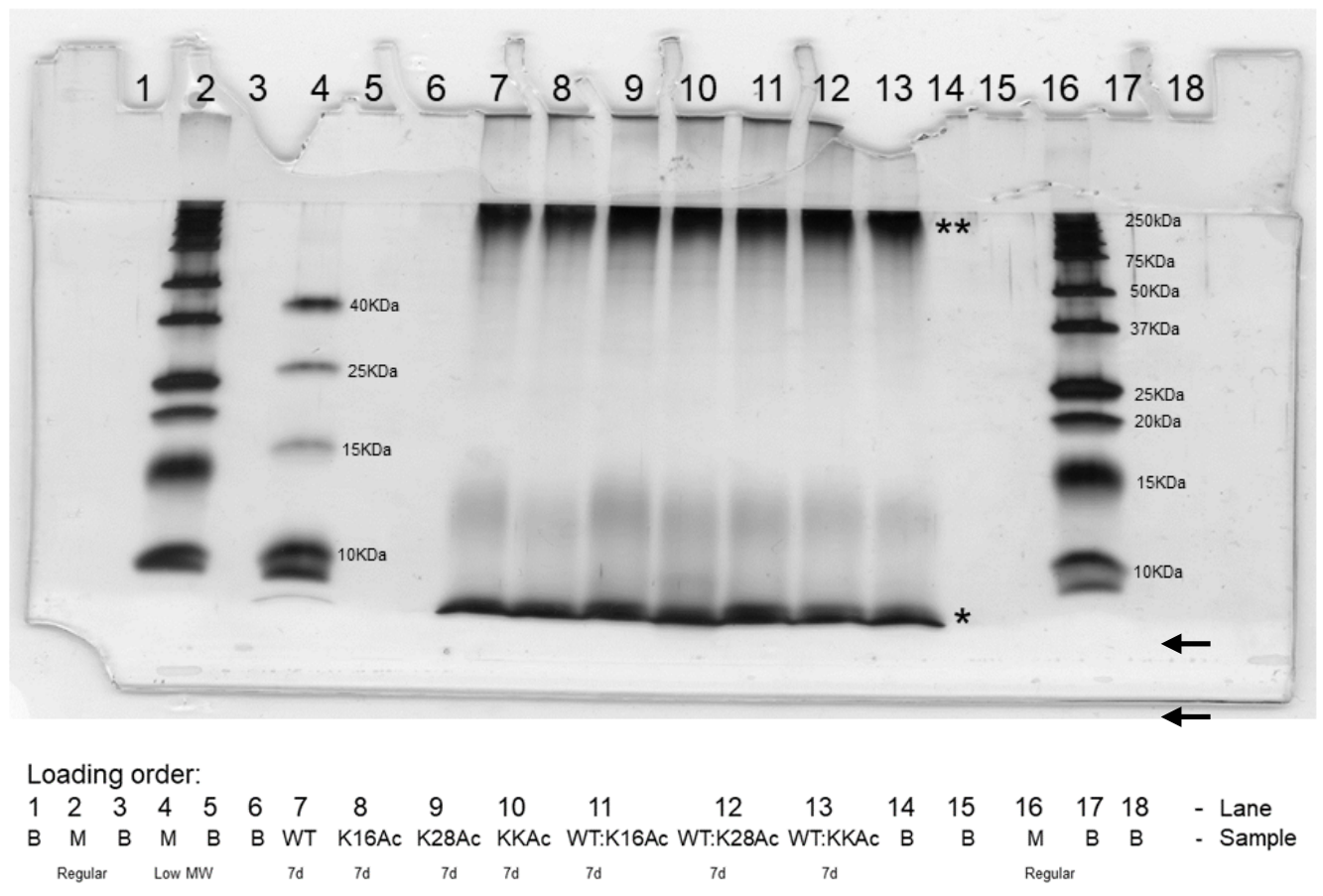

Supplementary Figure 17. Full-size gel image of silver staining of $7 \mathrm{~d}$ incubated WT, acetylated, and mixture of A $\beta 42$ peptides. B- blank, M-Marker, * represents monomer and ** represents high molecular weight. $(\leftarrow)$ represents the oligomeric species of varying molecular weight. 
Supplementary Table 1. Calculated surface area and hydrophobic patch values of WT and acetylated (K16Ac, K28 Ac and KKAc) aggregates obtained using SPDB software. Hydrophobic patch values below $100 \AA^{2}$ are not reported.

\begin{tabular}{|c|c|c|c|c|}
\hline Parameters & WT & K16Ac & $\mathrm{K} 28 \mathrm{Ac}$ & KKAc \\
\hline \multirow{2}{*}{$\begin{array}{l}\text { Surface area } \\
\left(\AA^{2}\right)\end{array}$} & 19199 & 20209 & 19703 & 19582 \\
\hline & 579 & 219 & 248 & 223 \\
\hline \multirow{11}{*}{$\begin{array}{l}\text { Hydrophobic } \\
\text { patch area } \\
\left(\AA^{2}\right)\end{array}$} & 278 & 208 & 200 & 196 \\
\hline & 243 & 193 & 199 & 143 \\
\hline & 220 & 173 & 179 & 133 \\
\hline & 189 & 151 & 153 & 130 \\
\hline & 181 & 143 & 140 & 121 \\
\hline & 164 & 136 & 138 & 121 \\
\hline & 118 & 122 & 125 & 120 \\
\hline & 109 & 120 & 110 & 115 \\
\hline & 105 & 117 & 105 & 111 \\
\hline & - & 104 & 101 & - \\
\hline & - & - & 101 & - \\
\hline
\end{tabular}

\section{Simulation systems.}

Initial structural configuration of WT A $\beta 42$ monomer with sequence

DAEFRHDSGYEVHHQKLVFFAEDV GSNKGAIIGLMVGGVVIA was taken from the PDB entry 1Z0Q. For MD simulations of WT and acetylated (K16Ac, K28Ac, and KKAc) A $\beta 42$ monomer, the peptide was first placed in a periodic box having dimension of $61.90 \times$ $60.00 \times 60.00 \AA^{3}$. The simulation box was uniformly solvated with water molecules with explicit waters defined using the TIP3P water model. Counter ions were added to the system to maintain charge neutralization. The solvated peptide system (WT and acetylated) was subjected to 200 steps of the energy minimization at a time step of 1 fs followed by $100 \mathrm{~ns}$ of production run.

To simulate the assembly and fibrillization process of 9 strands of WT and acetylated $A \beta 42$ peptide aggregates in aqueous solution, initial structure was taken to be the 9 monomer strands aligned. The peptides were then placed in the periodic box having dimension of $85.00 \times$ $80.00 \times 85.00 \AA^{3}$ and solvated with water molecules with the TIP3P water model. The total number of atoms and number of water molecules for all systems are provided in Table S2. Counterions were added to maintain charge neutralization in the system. The solvated peptides (WT and acetylated) was subjected to 200 steps of the energy minimization at a time step of $1 \mathrm{fs}$ followed by $100 \mathrm{~ns}$ of production run.

All simulations were performed at $310 \mathrm{~K}$ temperature and $101.3 \mathrm{kPa}$ pressure in the NPT ensemble using NAMD program and CHARMM27 force field. The 
experiments were performed at $310 \mathrm{~K}$. Data were saved every 20 ps intervals for analysis of the structural changes for 100 ns of MD trajectory. Convergence of the simulations was further confirmed using the root mean-square deviation (RMSD) analysis, implemented in VMD 1.9.2. suite of program. In addition, electrostatic potential isosurface for WT and acetylated peptides were computed using VMD program. Surface hydrophobic patches of WT and acetylated peptides corresponding to the structures at $100 \mathrm{~ns}$, were mapped on the molecular surface for the 4 systems using the SPDB program. 
Supplementary Table 2. Number of water molecules and total number of atoms in WT and acetylated (K16Ac, K28Ac, and KKAc) A $\beta 42$ monomer and peptide aggregates for MD simulations in aqueous solution.

\begin{tabular}{|c|c|c|c|c|c|}
\hline & & WT & K16Ac & K28Ac & KKAc \\
\cline { 2 - 6 } & \# total atoms & 20868 & 21728 & 20873 & 20779 \\
\cline { 2 - 6 } monomer & $\begin{array}{c}\text { \# water } \\
\text { molecules }\end{array}$ & 20238 & 21093 & 20238 & 20139 \\
\cline { 2 - 6 } & $\begin{array}{c}\text { \# A } 342 \\
\text { monomer }\end{array}$ & 627 & 631 & 631 & 635 \\
\cline { 2 - 6 } & $\begin{array}{c}\text { \# } \\
\text { counterions }\end{array}$ & 3 & 4 & 4 & 5 \\
\hline
\end{tabular}

\begin{tabular}{|c|l|c|c|c|c|}
\hline & & WT & K16Ac & K28Ac & KKAc \\
\cline { 2 - 6 } & \# total atoms & 58893 & 49486 & 58770 & 58824 \\
\cline { 2 - 6 } $\begin{array}{c}\text { 9 strands of } \\
\text { A } \beta 42 \\
\text { peptides }\end{array}$ & $\begin{array}{l}\text { \# water } \\
\text { molecules }\end{array}$ & 53222 & 43776 & 53055 & 53064 \\
\cline { 2 - 6 } & $\begin{array}{l}\# \text { A } \beta 42 \\
\text { monomer }\end{array}$ & 5643 & 5679 & 5679 & 5715 \\
\cline { 2 - 6 } & \# & & & & \\
\hline
\end{tabular}




\section{Statistical Analysis of $7 \mathrm{~d}$ samples}

One-way ANOVA was used for the statistics calculation of Fluorescence experiments (ANS, Bis-ANS) and toxicity assays (MTS, LDH, and DCF-HDA) carried out in SH-SY5Y and primary neuronal cells. Statistics for all 7 days data are provided below.

A. Statistical Analysis of ANS data. All acetylated and 1:1 mixture of A $\beta 427 d$ data were compared with WT of $7 \mathrm{~d}$. $\mathrm{p}<0.05$ was considered as significant and represented as *, $\mathrm{p}<0.005$ represented as $* *$ and $\mathrm{p}<0.0005$ represented as $* * *$ (Refer to figure 5 in chapter 4)

\section{WT vs K16Ac- 7d}

Anova: Single Factor

SUMMARY

\begin{tabular}{|c|c|c|c|c|c|c|}
\hline Groups & Count & Sum & Average & Variance & & \\
\hline & & 54710 & 18236.666 & 858433.333 & & \\
\hline \multirow{2}{*}{ WT } & 3 & 0 & 7 & 3 & & \\
\hline & & & & 701633.333 & & \\
\hline K16Ac & 3 & 96450 & 32150 & 3 & & \\
\hline \multicolumn{7}{|l|}{ ANOVA } \\
\hline \multicolumn{7}{|l|}{ Source of } \\
\hline Between & & & & 372.254946 & 4.25336E- & \\
\hline Groups & $2.9 \mathrm{E}+08$ & 1 & 29037126 & 4 & 05 & 7.70864 \\
\hline Within Groups & 3120133 & 4 & 780033.33 & & & \\
\hline & $2.93 E+0$ & & & & & \\
\hline Total & 8 & 5 & & & & \\
\hline
\end{tabular}




\section{WT vs K28Ac-7d}

Anova: Single Factor

SUMMARY

\begin{tabular}{|c|c|c|c|c|c|c|}
\hline Groups & Count & Sum & Average & Variance & & \\
\hline WT & 3 & 54710 & 18236.67 & 858433.3 & & \\
\hline $\mathrm{K} 28 \mathrm{AC}$ & 3 & 67330 & 22443.33 & 5549200 & & \\
\hline \multicolumn{7}{|l|}{ ANOVA } \\
\hline $\begin{array}{l}\text { Source of } \\
\text { Variation }\end{array}$ & SS & $d f$ & $M S$ & $F$ & P-value & F crit \\
\hline \multicolumn{7}{|l|}{ Between } \\
\hline Within Groups & 12815267 & 4 & 3203817 & & & \\
\hline Total & 39359333 & 5 & & & & \\
\hline
\end{tabular}

\section{WT vs KKAc-7d}

Anova: Single Factor

SUMMARY

\begin{tabular}{ccccc}
\hline Groups & Count & Sum & Average & Variance \\
\hline WT & 3 & 54710 & 18236.67 & 858433.3 \\
KKAc & 3 & 86130 & 28710 & 6242533 \\
\hline
\end{tabular}

ANOVA

Source of

Variation

SS $\quad d f \quad M S$




\begin{tabular}{lllllll}
$\begin{array}{l}\text { Between } \\
\text { Groups }\end{array}$ & $1.65 \mathrm{E}+08$ & 1 & $1.65 \mathrm{E}+08$ & 46.34188 & 0.002433 & 7.708647 \\
Within Groups & 14201933 & 4 & 3550483 & & \\
& & & & & \\
Total & $1.79 \mathrm{E}+08$ & 5 & & & \\
\hline
\end{tabular}

\section{WT vs WT:K16Ac-7d}

Anova: Single Factor

SUMMARY

\begin{tabular}{|c|c|c|c|c|c|c|}
\hline Groups & Count & Sum & Average & Variance & & \\
\hline WT & 3 & 54710 & 18236.67 & 858433.3 & & \\
\hline WT:K16Ac & 3 & 70640 & 23546.67 & 3206233 & & \\
\hline \multicolumn{7}{|l|}{ ANOVA } \\
\hline \multicolumn{7}{|l|}{ Source of } \\
\hline \multicolumn{7}{|l|}{ Between } \\
\hline Groups & 42294150 & 1 & 42294150 & 20.81064 & 0.010324 & 7.708647 \\
\hline Within Groups & 8129333 & 4 & 2032333 & & & \\
\hline Total & 50423483 & 5 & & & & \\
\hline
\end{tabular}

WT vs WT: K28Ac-7d

Anova: Single Factor

SUMMARY 


\begin{tabular}{lrrrr}
\hline \multicolumn{1}{c}{ Groups } & Count & Sum & Average & Variance \\
\hline WT & 3 & 54710 & 18236.6667 & 858433.3333 \\
WT:K28AC & 3 & 72040 & 24013.3333 & 6580800 \\
\hline
\end{tabular}

ANOVA

\begin{tabular}{lcccccc}
\hline $\begin{array}{l}\text { Source of } \\
\text { Variation }\end{array}$ & SS & $d f$ & MS & $F$ & P-value & Fcrit \\
\hline $\begin{array}{l}\text { Between } \\
\text { Groups }\end{array}$ & 5005481 & 1 & 50054816 & 13.4569825 & 0.02142173 & 7.70864 \\
Within & & & & & & \\
Groups & 1487846 & 4 & 3719616.6 & & & \\
& & & & & & \\
Total & 6493328 & 5 & & & & \\
\hline
\end{tabular}

WT vs WT: KKAc- 7d

Anova: Single Factor

SUMMARY

\begin{tabular}{ccccc}
\hline Groups & Count & Sum & Average & Variance \\
\hline WT & 3 & 54710 & 18236.67 & 858433.3 \\
WT:KKAC & 3 & 67060 & 22353.33 & 3204100 \\
\hline
\end{tabular}

ANOVA

\begin{tabular}{lcccccc}
\hline $\begin{array}{l}\text { Source of } \\
\text { Variation }\end{array}$ & SS & $d f$ & MS & $F$ & P-value & F crit \\
\hline $\begin{array}{l}\text { Between } \\
\text { Groups }\end{array}$ & 25420417 & 1 & 25420417 & 12.51456 & 0.024065 & 7.708647 \\
& & & & & & \\
& & & & & & \\
& & & & & &
\end{tabular}




$\begin{array}{lrrr}\text { Within Groups } & 8125067 & 4 & 2031267 \\ \text { Total } & 33545483 & 5 & \end{array}$

B. Statistical Analysis of Bis-ANS data. All acetylated and 1:1 mixtures of A $\beta 427 \mathrm{~d}$ data were compared with WT of $7 \mathrm{~d} . \mathrm{p}<0.05$ was considered as significant and represented as $*, p<0.005$ represented as $* *$ and $p<0.0005$ represented as $* * *$ (Refer to figure 3 in chapter 4)

\section{WT vs K16Ac-7d}

Anova: Single Factor

SUMMARY

\begin{tabular}{|c|c|c|c|c|c|c|}
\hline Groups & Count & Sum & Average & Variance & & \\
\hline \multirow[t]{2}{*}{ WT } & 3 & 363680 & 121226.666 & $1.2 E+08$ & & \\
\hline & 3 & 445230 & 148410 & $\begin{array}{c}1.59 \mathrm{E}+0 \\
8\end{array}$ & & \\
\hline \multicolumn{7}{|l|}{ ANOVA } \\
\hline $\begin{array}{l}\text { Source of } \\
\text { Variation }\end{array}$ & SS & $d f$ & MS & $F$ & $P$-value & F crit \\
\hline Between & & & & 7.95500 & 0.04780 & 7.70864 \\
\hline Groups & 1108400417 & 1 & 1108400417 & 8 & 6 & 7 \\
\hline Within & 557334666 . & & 139333666. & & & \\
\hline Groups & 7 & 4 & 7 & & & \\
\hline Total & 1665735083 & 5 & & & & \\
\hline
\end{tabular}

WT vs K28Ac- 7d 
Anova: Single Factor

SUMMARY

\begin{tabular}{|c|c|c|c|c|c|c|}
\hline Groups & Count & Sum & Average & Variance & & \\
\hline WT & 3 & 363680 & 121226.7 & $1.2 \mathrm{E}+08$ & & \\
\hline $\mathrm{K} 28 \mathrm{Ac}$ & 3 & 336100 & 112033.3 & 56194433 & & \\
\hline \multicolumn{7}{|l|}{ ANOVA } \\
\hline $\begin{array}{l}\text { Source of } \\
\text { Variation }\end{array}$ & SS & $d f$ & $M S$ & $F$ & P-value & F crit \\
\hline \multicolumn{7}{|l|}{ Between } \\
\hline Within Groups & $3.52 E+08$ & 4 & 88074233 & & & \\
\hline Total & $4.79 E+08$ & 5 & & & & \\
\hline
\end{tabular}

WT vs KKAc- 7d

Anova: Single Factor

SUMMARY

\begin{tabular}{|c|c|c|c|c|}
\hline Groups & Count & Sum & Average & Variance \\
\hline WT & 3 & 363680 & 121226.6667 & $1.2 \mathrm{E}+08$ \\
\hline KKAC & 3 & 408830 & 136276.6667 & 1539064 \\
\hline
\end{tabular}

Anova: Single Factor

ANOVA

\begin{tabular}{ccccccc}
$\begin{array}{c}\text { Source } \\
\text { of }\end{array}$ & SS & $d f$ & $M S$ & $F$ & P-value & Fcrit \\
\hline
\end{tabular}




\begin{tabular}{|c|c|c|c|c|c|c|}
\hline $\begin{array}{c}\text { Variatio } \\
n\end{array}$ & & & & & & \\
\hline \multicolumn{7}{|l|}{ Betwee } \\
\hline$n$ & 33975375 & & 33975375 & 5.59297 & 0.07724 & 7.70864 \\
\hline Groups & 0 & 1 & 0 & 2 & 5 & 7 \\
\hline Within & 24298619 & & 60746548. & & & \\
\hline \multirow[t]{2}{*}{ Groups } & 5.3 & 4 & 83 & & & \\
\hline & 58273994 & & & & & \\
\hline Total & 5.3 & 5 & & & & \\
\hline
\end{tabular}

WT vs WT:K16Ac- 7d

SUMMARY

\begin{tabular}{|c|c|c|c|c|}
\hline Groups & Count & Sum & Average & Variance \\
\hline WT & 3 & 363680 & 121226.7 & $1.2 \mathrm{E}+08$ \\
\hline WT:K16 & & & & $1.33 E+0$ \\
\hline Ac & 3 & 365120 & 121706.7 & 8 \\
\hline
\end{tabular}

ANOVA

\begin{tabular}{|c|c|c|c|c|c|c|}
\hline $\begin{array}{c}\text { Source } \\
\text { of } \\
\text { Variatio } \\
n\end{array}$ & SS & $d f$ & $M S$ & $F$ & $P$-value & F crit \\
\hline Betwee & & & & ברכתח ח & & 770861 \\
\hline Groups & 345600 & 1 & 345600 & 6 & 0.960792 & 7 \\
\hline $\begin{array}{l}\text { Within } \\
\text { Groups }\end{array}$ & $5.05 E+08$ & 4 & $1.26 \mathrm{E}+08$ & & & \\
\hline Total & $5.06 \mathrm{E}+08$ & 5 & & & & \\
\hline
\end{tabular}




\section{WT vs WT:K28Ac}

Anova: Single Factor

SUMMARY

\begin{tabular}{|c|c|c|c|c|c|c|}
\hline Groups & Count & Sum & Average & Variance & & \\
\hline WT & 3 & 363680 & 121226.7 & $1.2 \mathrm{E}+08$ & & \\
\hline WT:K28Ac & 3 & 386920 & 128973.3 & $2.07 E+08$ & & \\
\hline \multicolumn{7}{|l|}{ ANOVA } \\
\hline \multicolumn{7}{|l|}{ Source of } \\
\hline \multicolumn{7}{|l|}{ Between } \\
\hline Groups & 90016267 & 1 & 90016267 & 0.55121 & 0.499055 & 7.708647 \\
\hline Within Groups & $6.53 E+08$ & 4 & $1.63 E+08$ & & & \\
\hline Total & $7.43 E+08$ & 5 & & & & \\
\hline
\end{tabular}

WT vs WT:KKAc-7d

Anova: Single Factor

SUMMARY

\begin{tabular}{lrrrr}
\multicolumn{1}{c}{ Groups } & Count & Sum & Average & Variance \\
\hline \multirow{2}{*}{ WT } & 3 & 363680 & 121226.7 & $1.2 \mathrm{E}+08$ \\
WT:KKAc & 3 & 382380 & 127460 & $1.1 \mathrm{E}+08$ \\
\hline
\end{tabular}

ANOVA 


\begin{tabular}{lccccccc}
\hline $\begin{array}{l}\text { Source of } \\
\text { Variation }\end{array}$ & SS & $d f$ & MS & $F$ & P-value & F crit \\
\hline $\begin{array}{l}\text { Between } \\
\text { Groups }\end{array}$ & 58281667 & 1 & 58281667 & 0.507399 & 0.515612 & 7.708647 \\
Within Groups & $4.59 \mathrm{E}+08$ & 4 & $1.15 \mathrm{E}+08$ & & & \\
& & & & & & & \\
Total & $5.18 \mathrm{E}+08$ & 5 & & & & & \\
\hline
\end{tabular}

C. Statistical Analysis of MTS $(5 \mu \mathrm{M})$ data carried out in SH-SY5Y cells. WT, acetylated and 1:1 mixtures of $\mathrm{A} \beta 427 \mathrm{~d}$ data were compared with untreated cells which was used as control. $\mathrm{p}<0.05$ was considered as significant and represented as $*, \mathrm{p}<0.005$ represented as $* *$ and $\mathrm{p}<0.0005$ represented as $* * *$ (Refer to figure 6 in chapter 4)

\section{Untreated vs WT-7d}

Anova: Single Factor

SUMMARY

\begin{tabular}{lrrrr}
\hline Groups & Count & Sum & Average & Variance \\
\hline Untreated & 6 & 0.279 & 0.0465 & 0.000212 \\
WT & 6 & 0.187 & 0.031167 & 0.000163 \\
\hline
\end{tabular}

ANOVA

\begin{tabular}{lccccccc}
$\begin{array}{l}\text { Source of } \\
\text { Variation }\end{array}$ & SS & $d f$ & $M S$ & $F$ & P-value & F crit \\
\hline $\begin{array}{l}\text { Between } \\
\text { Groups }\end{array}$ & 0.000705 & 1 & 0.000705 & 3.756435 & 0.081329 & 4.964603 \\
Within Groups & 0.001878 & 10 & 0.000188 & & &
\end{tabular}




\begin{tabular}{|c|c|c|c|c|c|c|}
\hline Total & 0.002583 & 11 & & & & \\
\hline \multicolumn{7}{|c|}{ Untreated vs K16Ac-7d } \\
\hline \multicolumn{7}{|c|}{ Anova: Single Factor } \\
\hline \multicolumn{7}{|l|}{ SUMMARY } \\
\hline Groups & Count & Sum & Average & Variance & & \\
\hline Untreated & 6 & 0.279 & 0.0465 & 0.000212 & & \\
\hline K16Ac & 6 & 0.203 & 0.033833 & $2.02 \mathrm{E}-05$ & & \\
\hline \multicolumn{7}{|l|}{ ANOVA } \\
\hline \multicolumn{7}{|l|}{ Source of } \\
\hline \multicolumn{7}{|l|}{ Between } \\
\hline Groups & 0.000481 & 1 & 0.000481 & 4.143472 & 0.06916 & 4.964603 \\
\hline Within Groups & 0.001162 & 10 & 0.000116 & & & \\
\hline Total & 0.001643 & 11 & & & & \\
\hline
\end{tabular}

\section{Untreated vs K28Ac-7d}

Anova: Single Factor

SUMMARY

\begin{tabular}{lrrrr}
\hline Groups & Count & Sum & Average & Variance \\
\hline Untreated & 6 & 0.279 & 0.0465 & 0.000212 \\
K28Ac & 6 & 0.199 & 0.033167 & $2.58 \mathrm{E}-05$ \\
\hline
\end{tabular}




\begin{tabular}{lcccccc} 
ANOVA & & & & & & \\
\hline $\begin{array}{l}\text { Source of } \\
\text { Variation }\end{array}$ & SS & $d f$ & $M S$ & $F$ & P-value & F crit \\
\hline $\begin{array}{l}\text { Between } \\
\text { Groups }\end{array}$ & 0.000533 & 1 & 0.000533 & 4.483048 & 0.060295 & 4.964603 \\
Within Groups & 0.00119 & 10 & 0.000119 & & & \\
& & & & & & \\
Total & 0.001723 & 11 & & & & \\
\hline
\end{tabular}

Untreated vs KKAc-7d

Anova: Single Factor

SUMMARY

\begin{tabular}{|c|c|c|c|c|c|c|}
\hline Groups & Count & Sum & Average & Variance & & \\
\hline Untreated & 6 & 0.279 & 0.0465 & 0.000212 & & \\
\hline KKAC & 6 & 0.122 & 0.020333 & 0.000494 & & \\
\hline \multicolumn{7}{|l|}{ ANOVA } \\
\hline \multicolumn{6}{|l|}{ Source of } & F crit \\
\hline \multicolumn{7}{|l|}{ Between } \\
\hline Within Groups & 0.003531 & 10 & 0.000353 & & & \\
\hline Total & 0.005585 & 11 & & & & \\
\hline
\end{tabular}




\section{Untreated vs WT:K16Ac-7d}

Anova: Single Factor

SUMMARY

\begin{tabular}{|c|c|c|c|c|c|c|}
\hline Groups & Count & Sum & Average & Variance & & \\
\hline & & & & 0.00021 & & \\
\hline \multirow[t]{2}{*}{ Untreated } & 6 & 0.279 & 0.0465 & 2 & & \\
\hline & & 0.17333 & 0.02888 & 0.00011 & & \\
\hline WT:K16Ac & 6 & 3 & 9 & 3 & & \\
\hline \multicolumn{7}{|l|}{ ANOVA } \\
\hline \multicolumn{6}{|l|}{ Source of } & F crit \\
\hline \multicolumn{7}{|l|}{ Between } \\
\hline Groups & 0.00093 & 1 & 0.00093 & 5.71889 & 0.03786 & 4.96460 \\
\hline Within Groups & 0.00162 & 10 & 0.00016 & & & \\
\hline Total & 0.00255 & 11 & & & & \\
\hline
\end{tabular}

\section{Untreated vs WT:K28Ac-7d}

Anova: Single Factor

SUMMARY

\begin{tabular}{crrrr}
\hline Groups & Count & Sum & Average & Variance \\
\hline Untreated & 6 & 0.279 & 0.0465 & 0.000212 \\
WT:K28Ac & 6 & 0.164 & 0.027333 & $2.24 \mathrm{E}-05$ \\
\hline
\end{tabular}

ANOVA 


\begin{tabular}{lcccccc}
\hline $\begin{array}{l}\text { Source of } \\
\text { Variation }\end{array}$ & SS & $d f$ & $M S$ & $F$ & P-value & F crit \\
\hline $\begin{array}{l}\text { Between } \\
\text { Groups }\end{array}$ & 0.001102 & 1 & 0.001102 & 9.39676 & 0.011934 & 4.964603 \\
Within Groups & 0.001173 & 10 & 0.000117 & & & \\
& & & & & & \\
Total & 0.002275 & 11 & & & & \\
\hline
\end{tabular}

\section{Untreated vs KKAc-7d}

Anova: Single Factor

SUMMARY

\begin{tabular}{|c|c|c|c|c|c|c|}
\hline Groups & Count & Sum & Average & Variance & & \\
\hline Untreated & 6 & 0.279 & 0.0465 & 0.000212 & & \\
\hline WT:KKAc & 6 & 0.1 & 0.016667 & $4.28 \mathrm{E}-05$ & & \\
\hline \multicolumn{7}{|l|}{ ANOVA } \\
\hline $\begin{array}{l}\text { Source of } \\
\text { Variation }\end{array}$ & SS & $d f$ & $M S$ & $F$ & P-value & F crit \\
\hline \multicolumn{7}{|l|}{ Between } \\
\hline Within Groups & 0.001275 & 10 & 0.000127 & & & \\
\hline Total & 0.003945 & 11 & & & & \\
\hline
\end{tabular}

D. Statistical Analysis of LDH $(5 \mu \mathrm{M})$ data carried out in SH-SY5Y cells. WT, acetylated and 1:1 mixtures of $A \beta 427 \mathrm{~d}$ data were compared with untreated cells 
which was used as control. $\mathrm{p}<0.05$ was considered as significant and represented as $*, \mathrm{p}<0.005$ represented as $* *$ and $\mathrm{p}<0.0005$ represented as $* * *$ (Refer to figure 6 in chapter 4)

\section{Untreated vs WT-7d}

Anova: Single Factor

SUMMARY

\begin{tabular}{lrrrr}
\hline \multicolumn{1}{c}{ Groups } & Count & Sum & Average & Variance \\
\hline Untreated & 6 & 0.183 & 0.0305 & 0.00171 \\
WT & 6 & 0.819 & 0.1365 & $9.35 \mathrm{E}-05$ \\
\hline
\end{tabular}

ANOVA

\begin{tabular}{lcrcccc}
\hline $\begin{array}{c}\text { Source of } \\
\text { Variation }\end{array}$ & SS & $d f$ & MS & $F$ & P-value & F crit \\
\hline Between Groups & 0.033708 & 1 & 0.033708 & 37.37719 & 0.000113623 & 4.964603 \\
Within Groups & 0.009018 & 10 & 0.000902 & & & \\
& & & & & & \\
Total & 0.042726 & 11 & & & & \\
\hline
\end{tabular}

\section{Untreated vs K16Ac-7d}

Anova: Single Factor

SUMMARY

\begin{tabular}{|c|c|c|c|c|c|c|}
\hline Groups & Count & Sum & Average & Variance & & \\
\hline \multirow[t]{2}{*}{ Untreated } & 6 & 0.183 & 0.0305 & 0.00171 & & \\
\hline & & & & 0.00195 & & \\
\hline $\mathrm{K} 16 \mathrm{Ac}$ & 6 & 1.104 & 0.184 & 6 & & \\
\hline \multicolumn{7}{|l|}{ ANOVA } \\
\hline $\begin{array}{l}\text { Source of } \\
\text { Variation }\end{array}$ & SS & $d f$ & $M S$ & $F$ & $P$-value & F crit \\
\hline
\end{tabular}




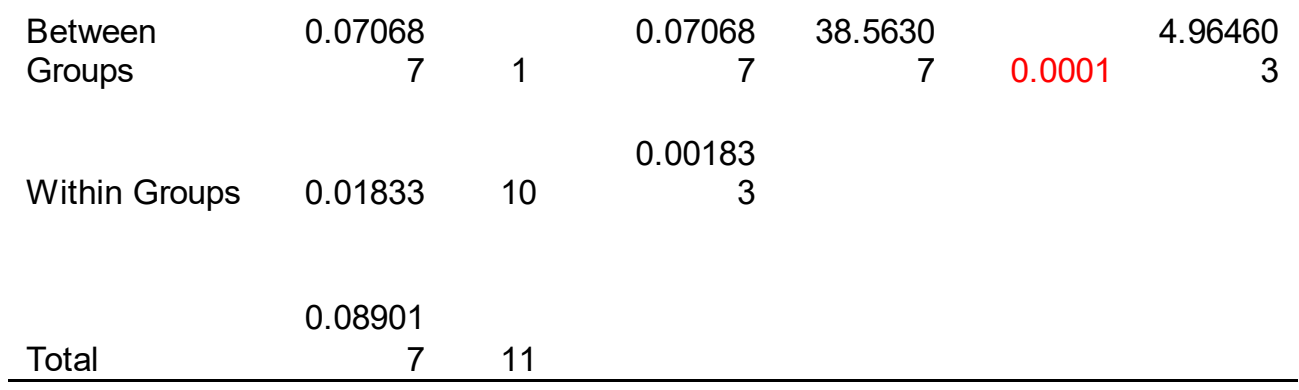

\section{Untreated vs K28Ac-7d}

Anova: Single Factor

SUMMARY

\begin{tabular}{|c|c|c|c|c|c|c|}
\hline Groups & Count & Sum & Average & Variance & & \\
\hline Untreated & 6 & 0.183 & 0.0305 & 0.00171016 & & \\
\hline $\mathrm{K} 28 \mathrm{Ac}$ & 6 & 0.971 & 0.16183 & 0.00204896 & & \\
\hline \multicolumn{7}{|l|}{ ANOVA } \\
\hline $\begin{array}{c}\text { Source of } \\
\text { Variation }\end{array}$ & SS & $d f$ & MS & $F$ & $P$-value & $F$ crit \\
\hline Between & & & & & 0.00037 & \\
\hline Groups & 0.05174 & 1 & 0.05174 & 27.5304591 & 5 & 4.96460274 \\
\hline Within Groups & 0.01879 & 10 & 0.00188 & & & \\
\hline Total & 0.07054 & 11 & & & & \\
\hline
\end{tabular}

\section{Untreated vs KKAc-7d}

Anova: Single Factor

SUMMARY

\begin{tabular}{lcccc}
\hline \multicolumn{1}{c}{ Groups } & Count & Sum & Average & Variance \\
\hline Untreated & 6 & 0.183 & 0.0305 & 0.00171 \\
KKAC & 6 & 1.617 & 0.2695 & 0.000489 \\
\hline
\end{tabular}

ANOVA 


\begin{tabular}{|c|c|c|c|c|c|c|}
\hline $\begin{array}{c}\text { Source of } \\
\text { Variation }\end{array}$ & SS & $d f$ & MS & $F$ & $P$-value & F crit \\
\hline Between & & & & & 2.0133E- & 4.96460 \\
\hline Groups & 0.171363 & 1 & 0.171363 & 155.8365 & 07 & 3 \\
\hline Within Groups & 0.010996 & 10 & 0.0011 & & & \\
\hline Total & 0.182359 & 11 & & & & \\
\hline
\end{tabular}

Untreated vs WT:K16Ac-

7d

Anova: Single Factor

SUMMARY

\begin{tabular}{|c|c|c|c|c|c|c|}
\hline Groups & Count & Sum & Average & Variance & & \\
\hline Untreated & 6 & 0.183 & 0.0305 & 0.00171 & & \\
\hline WT:K16Ac & 6 & 1.141 & 0.190167 & 0.002583 & & \\
\hline \multicolumn{7}{|l|}{ ANOVA } \\
\hline $\begin{array}{l}\text { Source of } \\
\text { Variation }\end{array}$ & SS & $d f$ & $M S$ & $F$ & $P$-value & $F$ crit \\
\hline Between Groups & 0.07648 & 1 & 0.07648 & 35.62914 & 0.000137691 & 4.964603 \\
\hline Within Groups & 0.021466 & 10 & 0.002147 & & & \\
\hline Total & 0.097946 & 11 & & & & \\
\hline
\end{tabular}

Untreated vs WT:K28Ac-

7d

Anova: Single Factor

SUMMARY

\begin{tabular}{|c|c|c|c|c|}
\hline Groups & Count & Sum & Average & Variance \\
\hline
\end{tabular}




\begin{tabular}{|c|c|c|c|c|c|c|}
\hline Untreated & 6 & 0.183 & 0.0305 & 0.00171 & & \\
\hline WT:K28Ac & 6 & 1.117 & 0.186167 & 0.002071 & & \\
\hline \multicolumn{7}{|l|}{ ANOVA } \\
\hline $\begin{array}{c}\text { Source of } \\
\text { Variation }\end{array}$ & $S S$ & $d f$ & $M S$ & $F$ & $P$-value & F crit \\
\hline Between Groups & 0.072696 & 1 & 0.072696 & 38.44807 & 0.000101 & 4.964603 \\
\hline Within Groups & 0.018908 & 10 & 0.001891 & & & \\
\hline Total & 0.091604 & 11 & & & & \\
\hline
\end{tabular}

\section{Untreated vs WT:KKAc-}

7d

Anova: Single Factor

SUMMARY

\begin{tabular}{lrrrr}
\hline Groups & Count & Sum & Average & Variance \\
\hline Untreated & 6 & 0.183 & 0.0305 & 0.00171 \\
WT:KKAc & 6 & 1.637 & 0.272833 & 0.0025 \\
\hline
\end{tabular}

ANOVA

\begin{tabular}{lcccccc}
\hline $\begin{array}{c}\text { Source of } \\
\text { Variation }\end{array}$ & SS & df & MS & $F$ & P-value & F crit \\
\hline Between Groups & 0.176176 & 1 & 0.176176 & 83.69289 & $3.5714 \mathrm{E}-06$ & 4.964603 \\
Within Groups & 0.02105 & 10 & 0.002105 & & & \\
& & & & & & \\
Total & 0.197227 & 11 & & & & \\
\hline
\end{tabular}

E. Statistical Analysis of DCFH-DA $(5 \mu \mathrm{M})$ data carried out in SHSY-5Y cells. WT, acetylated and 1:1 mixtures of $\mathrm{A} \beta 427 \mathrm{~d}$ data were compared with untreated cells 
which was used as control. $\mathrm{p}<0.05$ was considered as significant and represented as $*, \mathrm{p}<0.005$ represented as $* *$ and $\mathrm{p}<0.0005$ represented as $* * *$ (Refer to figure 6 in chapter 4)

\section{Untreated vs WT-7d}

Anova: Single Factor

SUMMARY

\begin{tabular}{lrrrr}
\hline Groups & Count & \multicolumn{1}{c}{ Sum } & Average & Variance \\
\hline & & & & \\
Untreated & 5 & 0.4361 & 0.08722 & 4 \\
& & & & \\
WT & & 1.08523 & 0.21704 & 0.00336 \\
& 5 & 3 & 7 & 2 \\
\hline
\end{tabular}

ANOVA

\begin{tabular}{lcccrrr}
\hline $\begin{array}{l}\text { Source of } \\
\text { Variation }\end{array}$ & SS & $d f$ & MS & $F$ & P-value & Fcrit \\
\hline $\begin{array}{l}\text { Between } \\
\text { Groups }\end{array}$ & 0.04213 & 1 & 0.04213 & 21.3556 & 0.00170748 & \\
Within & & & & & & \\
Groups & 0.01578 & 8 & 0.00197 & & & \\
Total & 0.05792 & 9 & & & & \\
\hline
\end{tabular}

\section{Untreated vs K16Ac-7d}

Anova: Single Factor

SUMMARY

\begin{tabular}{ccccc}
\hline Groups & Count & Sum & Average & Variance \\
\hline Untreated & 5 & 0.4361 & 0.08722 & 0.000584477 \\
& & & 217 &
\end{tabular}




\begin{tabular}{|c|c|c|c|c|c|c|}
\hline K16Ac & 5 & 2.2993 & 0.45986 & 0.000222993 & & \\
\hline ANOVA & & & & & & \\
\hline $\begin{array}{l}\text { Source of } \\
\text { Variation }\end{array}$ & SS & $d f$ & $M S$ & $F$ & P-value & F crit \\
\hline Between & & & & & $1.98174 \mathrm{E}-$ & \\
\hline Groups & 0.347151 & 1 & 0.347151424 & 859.8497133 & 09 & 5.317655 \\
\hline \multicolumn{7}{|l|}{ Within } \\
\hline Groups & 0.00323 & 8 & 0.000403735 & & & \\
\hline Total & 0.350381 & 9 & & & & \\
\hline
\end{tabular}

\section{Untreated vs K28Ac-7d}

Anova: Single Factor

SUMMARY

\begin{tabular}{|c|c|c|c|c|c|c|}
\hline Groups & Count & Sum & Average & Variance & & \\
\hline Untreated & 5 & 0.4361 & 0.08722 & 0.000584 & & \\
\hline $\mathrm{K} 28 \mathrm{AC}$ & 5 & 2.550633 & 0.510127 & 0.005405 & & \\
\hline \multicolumn{7}{|l|}{ ANOVA } \\
\hline $\begin{array}{l}\text { Source of } \\
\text { Variation }\end{array}$ & SS & $d f$ & $M S$ & $F$ & P-value & F crit \\
\hline Between & & & & & $1.86708 \mathrm{E}-$ & \\
\hline Groups & 0.447125 & 1 & 0.447125 & 149.3156 & 06 & 5.317655 \\
\hline Within Groups & 0.023956 & 8 & 0.002994 & & & \\
\hline Total & 0.471081 & 9 & & & & \\
\hline
\end{tabular}




\section{Untreated vs KKAc-7d}

Anova: Single Factor

SUMMARY

\begin{tabular}{|c|c|c|c|c|c|c|}
\hline Groups & Count & Sum & Average & Variance & & \\
\hline & & & & 0.00058447 & & \\
\hline \multirow[t]{2}{*}{ Untreated } & 5 & 0.4361 & 0.08722 & 7 & & \\
\hline & & 3.28833 & 0.65766 & & & \\
\hline KKAc & 5 & 3 & 7 & 0.0013063 & & \\
\hline \multicolumn{7}{|l|}{ ANOVA } \\
\hline \multicolumn{7}{|l|}{ Source of } \\
\hline Between & 0.81352 & & & & $1.97565 \mathrm{E}$ & 5.31765507 \\
\hline Groups & 3 & 1 & 0.81352 & 860.517658 & -09 & 2 \\
\hline \multicolumn{7}{|l|}{ Within } \\
\hline Groups & 0.00756 & 8 & 0.00094 & & & \\
\hline Total & 0.82108 & 9 & & & & \\
\hline
\end{tabular}

\section{Untreated vs WT:K16Ac-7d}

Anova: Single Factor

SUMMARY

\begin{tabular}{|c|c|c|c|c|}
\hline Groups & Count & Sum & Average & Variance \\
\hline Untreated & 5 & 0.4361 & 0.08722 & 0.00058447 \\
\hline & & 2.78883 & 0.55776 & \\
\hline WT:K16Ac & 5 & 3 & 7 & 0.0011343 \\
\hline
\end{tabular}




\begin{tabular}{|c|c|c|c|c|c|c|}
\hline $\begin{array}{l}\text { Source of } \\
\text { Variation }\end{array}$ & SS & $d f$ & $M S$ & $F$ & P-value & F crit \\
\hline Between & & & & 644.103817 & $6.22434 \mathrm{E}-$ & \\
\hline Groups & 0.55353 & 1 & 0.55353 & 7 & 09 & 5.31765 \\
\hline Within Groups & 0.00687 & 8 & 0.00085 & & & \\
\hline Total & 0.56041 & 9 & & & & \\
\hline
\end{tabular}

\section{Untreated vs WT:K28Ac-7d}

Anova: Single Factor

SUMMARY

\begin{tabular}{|c|c|c|c|c|c|c|}
\hline Groups & Count & Sum & Average & Variance & & \\
\hline Untreated & 5 & 0.4361 & 0.08722 & 0.00058447 & & \\
\hline WT:K28Ac & 5 & 2.53896 & 0.50779 & 0.00385373 & & \\
\hline \multicolumn{7}{|l|}{ ANOVA } \\
\hline \multicolumn{7}{|l|}{ Source of } \\
\hline Between & & & & & $6.16418 \mathrm{E}-$ & \\
\hline Groups & 0.44220 & 1 & 0.44220 & 199.271698 & 07 & 5.31765 \\
\hline Within Groups & 0.01775 & 8 & 0.00221 & & & \\
\hline Total & 0.45995 & 9 & & & & \\
\hline
\end{tabular}




\section{Untreated vs WT:KKAc-7d}

Anova: Single Factor

SUMMARY

\begin{tabular}{lrrrr}
\hline Groups & Count & Sum & Average & Variance \\
\hline Untreated & 5 & 0.4361 & 0.08722 & 0.000584 \\
WT:KKAC & 5 & 5.187167 & 1.037433 & 0.004266 \\
\hline
\end{tabular}

ANOVA

\begin{tabular}{|c|c|c|c|c|c|c|}
\hline $\begin{array}{l}\text { Source of } \\
\text { Variation }\end{array}$ & SS & $d f$ & MS & $F$ & P-value & F crit \\
\hline Between & & & & & $1.44778 \mathrm{E}-$ & \\
\hline Groups & 2.257263 & 1 & 2.257263 & 930.6428 & 09 & 5.317655 \\
\hline Within Groups & 0.019404 & 8 & 0.002425 & & & \\
\hline Total & 2.276667 & 9 & & & & \\
\hline
\end{tabular}

F. Statistical Analysis of $\mathrm{LDH}(2 \mu \mathrm{M})$ data carried out in primary neuronal cells. WT, acetylated and 1:1 mixtures of $A \beta 427 d$ data were compared with untreated cells which was used as control. $\mathrm{p}<0.05$ was considered as significant and represented as *, $\mathrm{p}<0.005$ represented as $* *$ and $\mathrm{p}<0.0005$ represented as $* * *$ (Refer to figure 7 in chapter 4)

\section{Untreated vs WT -7d}

Anova: Single Factor

SUMMARY 


\begin{tabular}{|c|c|c|c|c|c|c|}
\hline Groups & Count & Sum & Average & Variance & & \\
\hline Untreated & 4 & 1.200667 & 0.300167 & 0.001145 & & \\
\hline WT & 4 & 3.538667 & 0.884667 & 0.002106 & & \\
\hline \multicolumn{7}{|l|}{ ANOVA } \\
\hline $\begin{array}{c}\text { Source of } \\
\text { Variation }\end{array}$ & SS & $d f$ & MS & $F$ & $P$-value & F crit \\
\hline Between Groups & 0.683281 & 1 & 0.683281 & 420.351 & $\begin{array}{r}8.75603 \mathrm{E}- \\
07\end{array}$ & 5.987378 \\
\hline Within Groups & 0.009753 & 6 & 0.001626 & & & \\
\hline Total & 0.693034 & 7 & & & & \\
\hline
\end{tabular}

\section{Untreated vs K16Ac-7d}

Anova: Single Factor

SUMMARY

\begin{tabular}{lcccc}
\hline \multicolumn{1}{c}{ Groups } & Count & Sum & Average & Variance \\
\hline Untreated & 4 & 1.200667 & 0.300167 & 0.001145 \\
K16Ac & 4 & 6.187667 & 1.546917 & 0.004958 \\
\hline
\end{tabular}

ANOVA

\begin{tabular}{lccccrrr}
\hline $\begin{array}{c}\text { Source of } \\
\text { Variation }\end{array}$ & SS & $d f$ & MS & $F$ & P-value & F crit \\
\hline & & & & & & & \\
Between Groups & 3.108771 & 1 & 3.108771 & 1018.726 & $0868 \mathrm{E}-$ & 5.987378 \\
Within Groups & 0.01831 & 6 & 0.003052 & & & \\
& & & & & & \\
Total & 3.127081 & 7 & & & & \\
\hline
\end{tabular}




\section{Untreated vs K28Ac-}

7d

Anova: Single Factor

SUMMARY

\begin{tabular}{|c|c|c|c|c|c|c|}
\hline Groups & Count & Sum & Average & Variance & & \\
\hline Untreated & 4 & 1.200667 & 0.300166667 & 0.001145 & & \\
\hline $\mathrm{K} 28 \mathrm{Ac}$ & 4 & 4.781 & 1.19525 & 0.000308 & & \\
\hline \multicolumn{7}{|l|}{ ANOVA } \\
\hline $\begin{array}{c}\text { Source of } \\
\text { Variation }\end{array}$ & SS & $d f$ & MS & $F$ & $P$-value & F crit \\
\hline $\begin{array}{l}\text { Between } \\
\text { Groups }\end{array}$ & 1.602348 & 1 & 1.602348347 & 2206.205 & $\begin{array}{r}6.24123 \mathrm{E}- \\
09\end{array}$ & 5.987377607 \\
\hline $\begin{array}{l}\text { Within } \\
\text { Groups }\end{array}$ & 0.004358 & 6 & 0.000726292 & & & \\
\hline Total & 1.606706 & 7 & & & & \\
\hline
\end{tabular}

\section{Untreated vs KKAc-7d}

Anova: Single Factor

SUMMARY

\begin{tabular}{lrccc}
\hline \multicolumn{1}{c}{ Groups } & Count & Sum & Average & Variance \\
\hline Untreated & 4 & 1.200667 & 0.300167 & 0.001145 \\
KKAc & 4 & 8.228667 & 2.057167 & 0.004659 \\
\hline
\end{tabular}

ANOVA

\begin{tabular}{ccccccc}
$\begin{array}{c}\text { Source of } \\
\text { Variation }\end{array}$ & SS & $d f$ & $M S$ & $F$ & $P$-value & $F$ crit \\
\hline
\end{tabular}




\begin{tabular}{lrrrrrr}
$\begin{array}{l}\text { Between } \\
\text { Groups }\end{array}$ & 6.174098 & 1 & 6.174098 & 2127.532047 & $0.95769 \mathrm{E}-$ & \\
& & & & & \\
$\begin{array}{l}\text { Within } \\
\text { Groups }\end{array}$ & 0.017412 & 6 & 0.002902 & & \\
& & & & & \\
Total & 6.19151 & 7 & & & \\
\hline
\end{tabular}

\section{Untreated vs WT: K16Ac-7d}

Anova: Single Factor

SUMMARY

\begin{tabular}{|c|c|c|c|c|c|c|}
\hline Groups & Count & Sum & Average & Variance & & \\
\hline Untreated & 4 & 1.200667 & 0.300167 & 0.001145 & & \\
\hline WT:K16Ac & 4 & 6.220667 & 1.555167 & 0.004269667 & & \\
\hline \multicolumn{7}{|l|}{ ANOVA } \\
\hline $\begin{array}{l}\text { Source of } \\
\text { Variation }\end{array}$ & SS & $d f$ & MS & $F$ & $P$-value & $F$ crit \\
\hline $\begin{array}{l}\text { Between } \\
\text { Groups }\end{array}$ & 3.15005 & 1 & 3.15005 & 1163.524994 & $\begin{array}{r}4.22778 \mathrm{E}- \\
08\end{array}$ & 5.987377607 \\
\hline Within Groups & 0.016244 & 6 & 0.002707 & & & \\
\hline Total & 3.166294 & 7 & & & & \\
\hline
\end{tabular}

Untreated vs

WT:K28Ac-7d

Anova: Single Factor

SUMMARY

\begin{tabular}{ccccc}
\hline Groups & Count & Sum & Average & Variance \\
\hline Untreated & 4 & 1.200667 & 0.300167 & 0.001145 \\
& & & \multicolumn{2}{c}{224}
\end{tabular}




\begin{tabular}{|c|c|c|c|c|c|c|}
\hline WT:K28Ac & 4 & 6.021667 & 1.505417 & 0.003206 & & \\
\hline ANOVA & & & & & & \\
\hline $\begin{array}{c}\text { Source of } \\
\text { Variation }\end{array}$ & SS & $d f$ & MS & $F$ & $P$-value & $F$ crit \\
\hline $\begin{array}{l}\text { Between } \\
\text { Groups }\end{array}$ & 2.905255 & 1 & 2.905255 & 1335.366 & $\begin{array}{r}2.80151 \mathrm{E}- \\
08\end{array}$ & 5.987378 \\
\hline $\begin{array}{l}\text { Within } \\
\text { Groups }\end{array}$ & 0.013054 & 6 & 0.002176 & & & \\
\hline Total & 2.918309 & 7 & & & & \\
\hline
\end{tabular}

Untreated vs

WT:KKAc-7d

Anova: Single Factor

SUMMARY

\begin{tabular}{|c|c|c|c|c|c|c|}
\hline Groups & Count & Sum & Average & Variance & & \\
\hline Untreated & 4 & 1.200666667 & 0.300167 & 0.001145 & & \\
\hline WT:KKAc & 4 & 6.841666667 & 1.710417 & 0.00518825 & & \\
\hline \multicolumn{7}{|l|}{ ANOVA } \\
\hline $\begin{array}{c}\text { Source of } \\
\text { Variation }\end{array}$ & SS & $d f$ & MS & $F$ & $P$-value & $F$ crit \\
\hline $\begin{array}{l}\text { Between } \\
\text { Groups }\end{array}$ & 3.97761 & 1 & 3.97761 & 1256.103936 & $\begin{array}{r}3.36352 \mathrm{E}- \\
08\end{array}$ & 5.987378 \\
\hline $\begin{array}{l}\text { Within } \\
\text { Groups }\end{array}$ & 0.019 & 6 & 0.003167 & & & \\
\hline Total & 3.99661 & 7 & & & & \\
\hline
\end{tabular}


G. Statistics Analys is of DCFH-DA $(2 \mu \mathrm{M})$ data carried out in primary neuronal cells. WT, acetylated and 1:1 mixtures of $A \beta 427 \mathrm{~d}$ data were compared with untreated cells which was used as control. $\mathrm{p}<0.05$ was considered as significant and represented as $*, \mathrm{p}<0.005$ represented as $* *$ and $\mathrm{p}<0.0005$ represented as $* * *$ (Refer to figure 7 in chapter 4)

\section{Untreated vs WT- 7d}

Anova: Single Factor

SUMMARY

\begin{tabular}{lrrrr}
\hline \multicolumn{1}{c}{ Groups } & Count & Sum & Average & Variance \\
\hline Untreated & 5 & 0.3787 & 0.07574 & 0.000917 \\
WT & 5 & 0.964567 & 0.192913 & 0.002527 \\
\hline
\end{tabular}

ANOVA

\begin{tabular}{lcccccc}
\hline $\begin{array}{l}\text { Source of } \\
\text { Variation }\end{array}$ & SS & $d f$ & MS & $F$ & P-value & F crit \\
\hline $\begin{array}{l}\text { Between } \\
\text { Groups }\end{array}$ & 0.034324 & 1 & 0.034324 & 19.93725 & 0.002097 & 5.317655 \\
Within Groups & 0.013773 & 8 & 0.001722 & & & \\
& & & & & & \\
Total & 0.048097 & 9 & & & & \\
\hline
\end{tabular}

\section{Untreated vs K16Ac-7d}

Anova: Single Factor

SUMMARY

\begin{tabular}{cccc}
\hline Groups & Count & Sum & Average \\
& & \\
& &
\end{tabular}




\begin{tabular}{|c|c|c|c|c|c|c|}
\hline Untreated & 5 & 0.3787 & 0.07574 & 0.00091 & & \\
\hline K16Ac & 5 & 1.44263 & 0.28852 & 0.00499 & & \\
\hline \multicolumn{7}{|l|}{ ANOVA } \\
\hline $\begin{array}{l}\text { Source of } \\
\text { Variation }\end{array}$ & SS & $d f$ & $M S$ & $F$ & P-value & F crit \\
\hline \multicolumn{7}{|l|}{ Between } \\
\hline Groups & 0.11319 & 1 & 0.11319 & 38.2676 & 0.000263396 & 5.317655 \\
\hline Within Groups & 0.02366 & 8 & 0.00295 & & & \\
\hline Total & 0.13685 & 9 & & & & \\
\hline
\end{tabular}

Untreated vs K28Ac-7d

Anova: Single Factor

SUMMARY

\begin{tabular}{lrrrr}
\hline Groups & Count & Sum & Average & Variance \\
\hline Untreated & 5 & 0.3787 & 0.07574 & 0.000917 \\
K28AC & 5 & 1.068567 & 0.213713 & 0.003781 \\
\hline ANOVA & & & & \\
\hline
\end{tabular}

Source of

Variation

SS $\quad d f$

MS $\quad F$

P-value

F crit

Between

Groups

0.047592

$1 \quad 0.047592 \quad 20.26017 \quad 0.001999 \quad 5.317655$

Within Groups 0.018792

$\begin{array}{ll}8 & 0.002349\end{array}$ 


\section{Untreated vs KKAc -7d}

Anova: Single Factor

SUMMARY

\begin{tabular}{lrcrc}
\hline Groups & Count & Sum & Average & Variance \\
\hline Untreated & 5 & 0.3787 & 0.07574 & 0.000917 \\
KKAc & 5 & 2.192133 & 0.438427 & 0.004799 \\
\hline
\end{tabular}

ANOVA

\begin{tabular}{|c|c|c|c|c|c|c|}
\hline $\begin{array}{l}\text { Source of } \\
\text { Variation }\end{array}$ & SS & $d f$ & $M S$ & $F$ & P-value & F crit \\
\hline Between & & & & & 5.01524E- & \\
\hline Groups & 0.328854 & 1 & 0.328854 & 115.0654 & 06 & 5.317655 \\
\hline Within Groups & 0.022864 & 8 & 0.002858 & & & \\
\hline Total & 0.351718 & 9 & & & & \\
\hline
\end{tabular}

Untreated vs WT: K16Ac -7d

Anova: Single Factor

SUMMARY

\begin{tabular}{crccc}
\hline Groups & Count & Sum & Average & Variance \\
\hline Untreated & 5 & 0.3787 & 0.07574 & 0.000917 \\
WT:K16Ac & 5 & 1.604033 & 0.320807 & 0.000374 \\
\hline
\end{tabular}

ANOVA 


\begin{tabular}{|c|c|c|c|c|c|c|}
\hline $\begin{array}{l}\text { Source of } \\
\text { Variation }\end{array}$ & SS & $d f$ & $M S$ & $F$ & P-value & F crit \\
\hline Between & & & & & 3.37998E- & \\
\hline Groups & 0.150144 & 1 & 0.150144 & 232.7355 & 07 & 5.317655 \\
\hline Within Groups & 0.005161 & 8 & 0.000645 & & & \\
\hline Total & 0.155305 & 9 & & & & \\
\hline
\end{tabular}

Untreated vs K28Ac -7d

Anova: Single Factor

SUMMARY

\begin{tabular}{crrrr}
\hline Groups & Count & Sum & Average & Variance \\
\hline Untreated & 5 & 0.3787 & 0.07574 & 0.000917 \\
WT:K28AC & 5 & 1.52106 & 0.304213 & 0.000622 \\
\hline
\end{tabular}

ANOVA

\begin{tabular}{|c|c|c|c|c|c|c|}
\hline $\begin{array}{l}\text { Source of } \\
\text { Variation }\end{array}$ & SS & $d f$ & $M S$ & $F$ & P-value & F crit \\
\hline Between & & & & & 1.14564E- & \\
\hline Groups & 0.1305 & 1 & 0.1305 & 169.638 & 06 & 5.317655 \\
\hline Within Groups & 0.00615 & 8 & 0.00076 & & & \\
\hline Total & 0.13665 & 9 & & & & \\
\hline
\end{tabular}

Untreated vs WT: KKAc -7d

Anova: Single Factor 
SUMMARY

\begin{tabular}{|c|c|c|c|c|c|c|}
\hline Groups & Count & Sum & Average & Variance & & \\
\hline Untreated & 5 & 0.3787 & 0.07574 & 0.000916608 & & \\
\hline WT: KKAC & 5 & 2.2765 & 0.4553 & 0.004404 & & \\
\hline \multicolumn{7}{|l|}{ ANOVA } \\
\hline $\begin{array}{l}\text { Source of } \\
\text { Variation }\end{array}$ & SS & $d f$ & $M S$ & $F$ & P-value & F crit \\
\hline $\begin{array}{l}\text { Between } \\
\text { Groups }\end{array}$ & 0.36016 & 1 & 0.36016 & 135.3847094 & $\begin{array}{r}2.71104 \mathrm{E}- \\
06\end{array}$ & 5.317655 \\
\hline Within Groups & 0.02128 & 8 & 0.00266 & & & \\
\hline Total & 0.38144 & 9 & & & & \\
\hline
\end{tabular}




\section{Permission for figure 1 in Chapter 1}

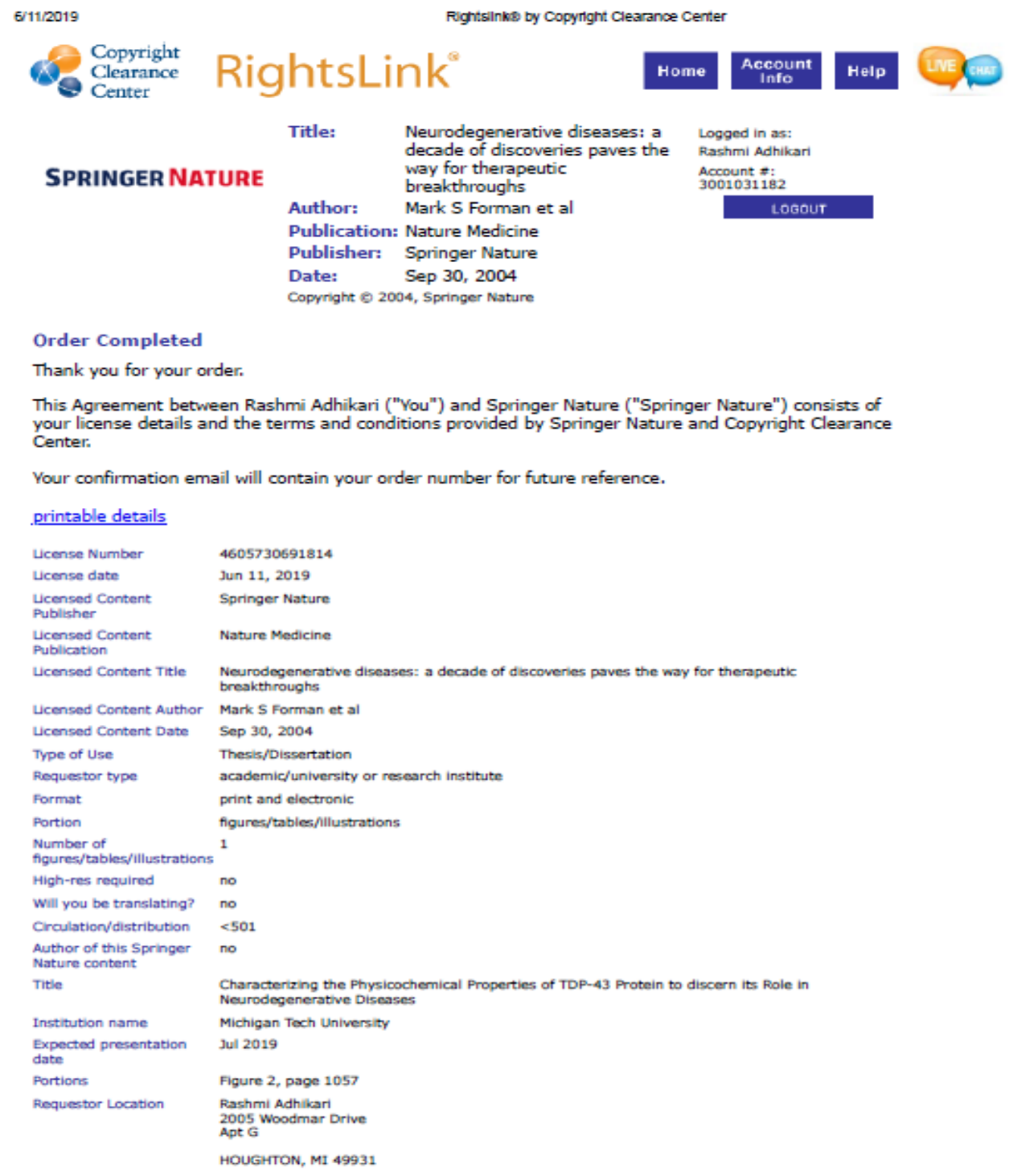




\section{Permission for figure 2 in Chapter 1}

\section{For permission for figures in dissertation (1) \\ 2 messages}

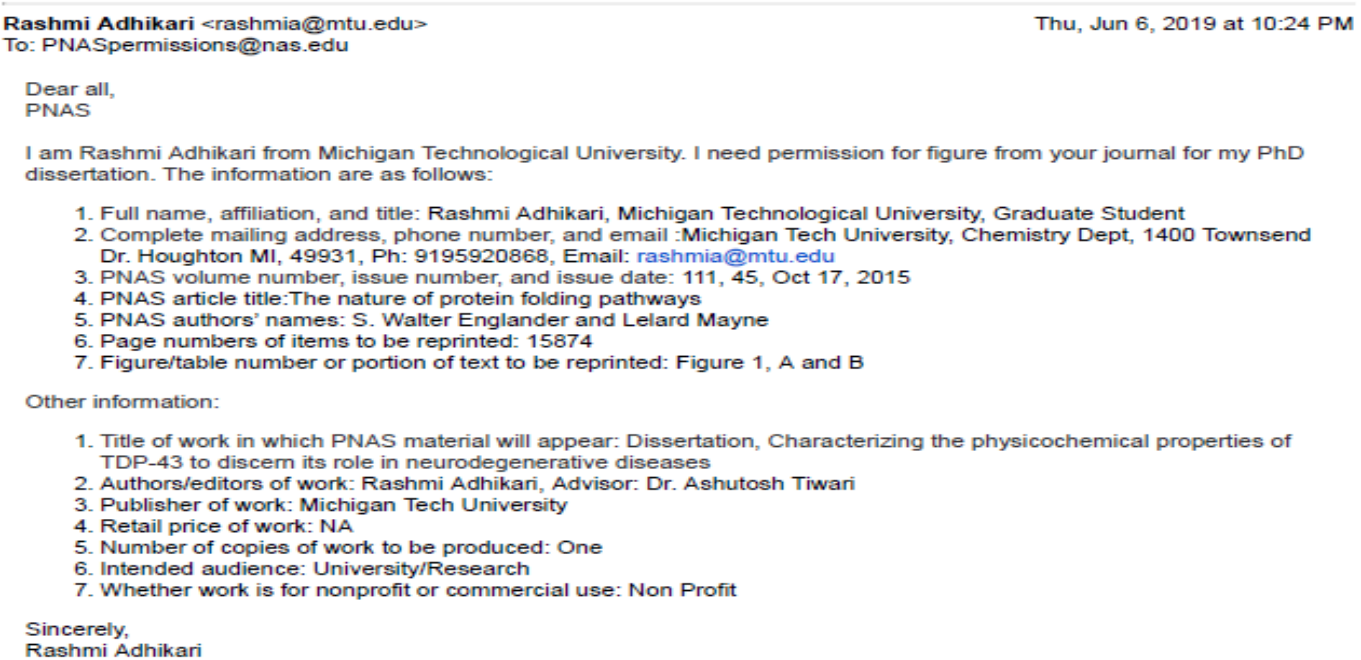

I am Rashmi Adhikari from Michigan Technological University. I need permission for figure from your journal for my PhD dissertation. The information are as follows:

1. Full name, affiliation, and title: Rashmi Adhikari, Michigan Technological University, Graduate Student

2. Complete mailing address, phone number, and email :Michigan Tech University, Chemistry Dept, 1400 Townsend

Dr. Houghton MI, 49931, Ph: 9195920868, Email: rashmia@mtu.edu

3. PNAS volume number, issue number, and issue date: 111,45 , Oct 17,2015

4. PNAS article title:The nature of protein folding pathways

5. PNAS authors' names: S. Walter Englander and Lelard Mayne

6. Page numbers of items to be reprinted: 15874

7. Figure/table number or portion of text to be reprinted: Figure 1, A and B

Other information:

1. Title of work in which PNAS material will appear: Dissertation, Characterizing the physicochemical properties of TDP-43 to discem its role in neurodegenerative diseases

2. Authors/editors of work: Rashmi Adhikari, Advisor: Dr. Ashutosh Tiwari

3. Publisher of work: Michigan Tech University

4. Retail price of work: NA

Number of copies of work to be produced: One

6. Intended audience: University/Research

7. Whether work is for nonprofit or commercial use: Non Profit

Sincerely,

Rashmi Adhikari

PNAS Permissions <PNASPermissions@nas.edu>

Fri, Jun 7, 2019 at 3:45 PM

To: Rashmi Adhikari <rashmia@mtu.edu>

Thank you for your message. PNAS can only grant permission for panel A of Figure 1. Please include a full citation for the original PNAS article when reusing the material. Because this material published after 2008, a copyright note is not needed. There is no charge for this material, either.

PNAS cannot grant permission for panel B of Figure 1, as we do not hold the copyright. The legend for Figure 1B states that the image was "Reprinted with permission from ref. 13."

Please contact the authors/publisher of ref. 13 for permission to use the image. Below is the citation information for the original publication. Let us know if you have any questions. 


\section{Permission for figure 3 in Chapter 1}

\section{For permission for figures in dissertation (2) \\ 2 messages}

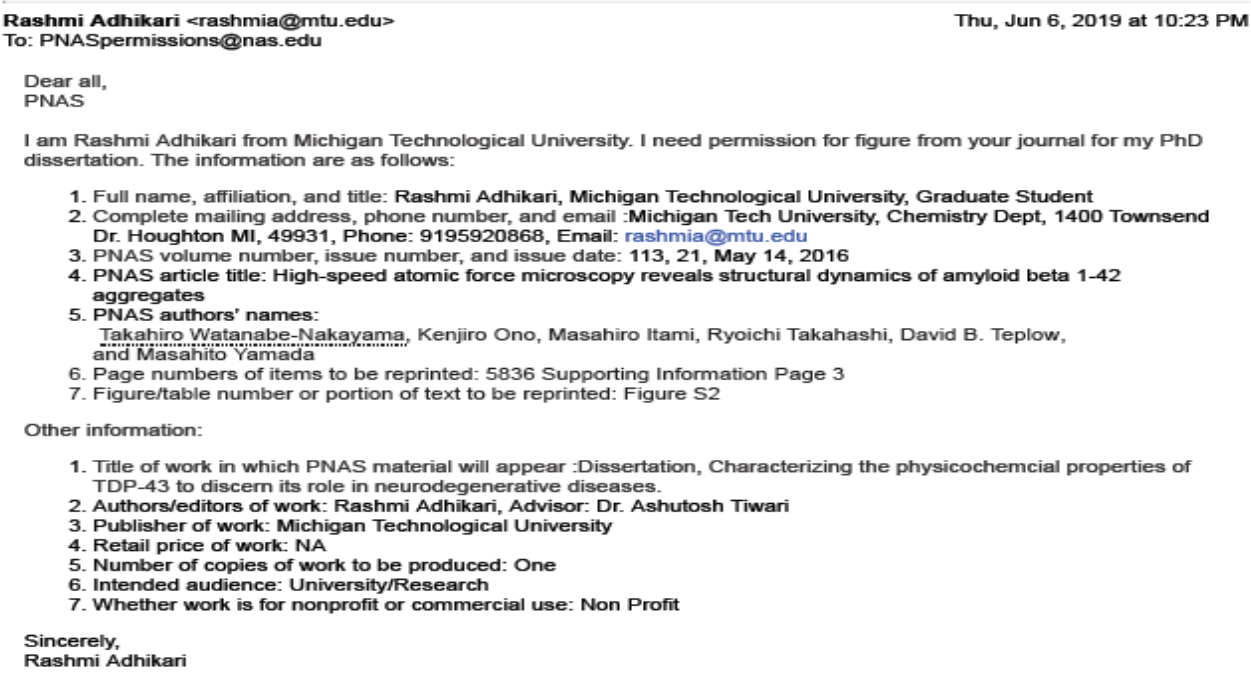

1. Full name, affiliation, and title: Rashmi Adhikari, Michigan Technological University, Graduate Student

1. Title of work in which PNAS material will appear :Dissertation, Characterizing the physicochemcial properties of 1. TDP-43 to discern its role in neurodegenerative diseases.

2. Authors/editors of work: Rashmi Adhikari, Advisor: Dr. Ashutosh Tiwari

3. Publisher of work: Michigan Technological University

4. Retail price of work: NA

5. Number of copies of work to be produced: One

6. Intended audience: University/Research

7. Whether work is for nonprofit or commercial use: Non Profit

Sincerely,

Rashmi Adhikari

PNAS Permissions <PNASPermissions@nas.edu>
To: Rashmi Adhikari <rashmia@mtu.edu>

Fri, Jun 7, 2019 at 3:49 PM

Thank you for your message. Permission is granted for your use of the material as described in your request. Please include a full citation for the original PNAS article when reusing the material. Because this material published after 2008 , a copyright note is not needed. There is no charge for this material, either. Let us know if you have any
questions.

Sincerely,

Kay McLaughlin for

Diane Sullenberger

PNAS Executive Editor 


\section{Permission for figure 4 in Chapter 1}

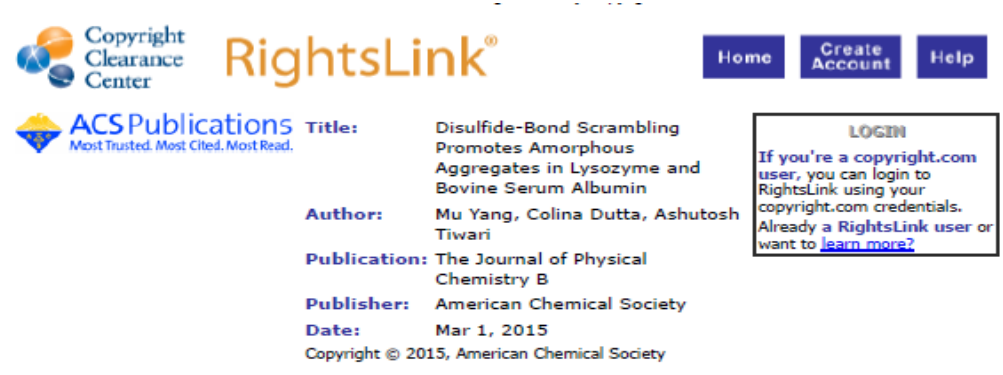

PERMISSION/LICENSE IS GRANTED FOR YOUR ORDER AT NO CHARGE

This type of permission/license, instead of the standard Terms \& Conditions, is sent to you because no fee is being charged for your order. Please note the following:

- Permission is granted for your request in both print and electronic formats, and translations.

- If figures and/or tables were requested, they may be adapted or used in part.

- Please print this page for your records and send a copy of it to your publisher/graduate

school.

- Appropriate credit for the requested material should be given as follows: "Reprinted (adapted) with permission from (COMPLETE REFERENCE CITATION). Copyright (YEAR) American Chemical Society." Insert appropriate information in place of the capitalized words.

- One-time permission is granted only for the use specified in your request. No additional uses are granted (such as derivative works or other editions). For any other uses, please submit a new request.

If credit is given to another source for the material you requested, permission must be obtained from that source. 
Permission for figure 3 in Chapter 2

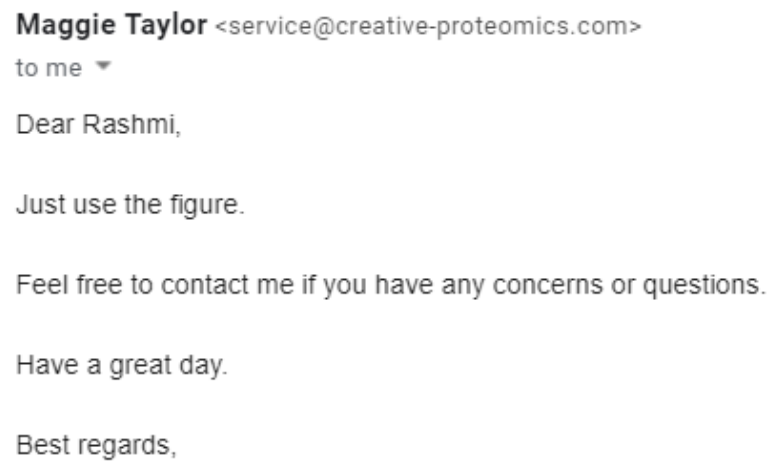


Permission for figure 2 in Chapter 3

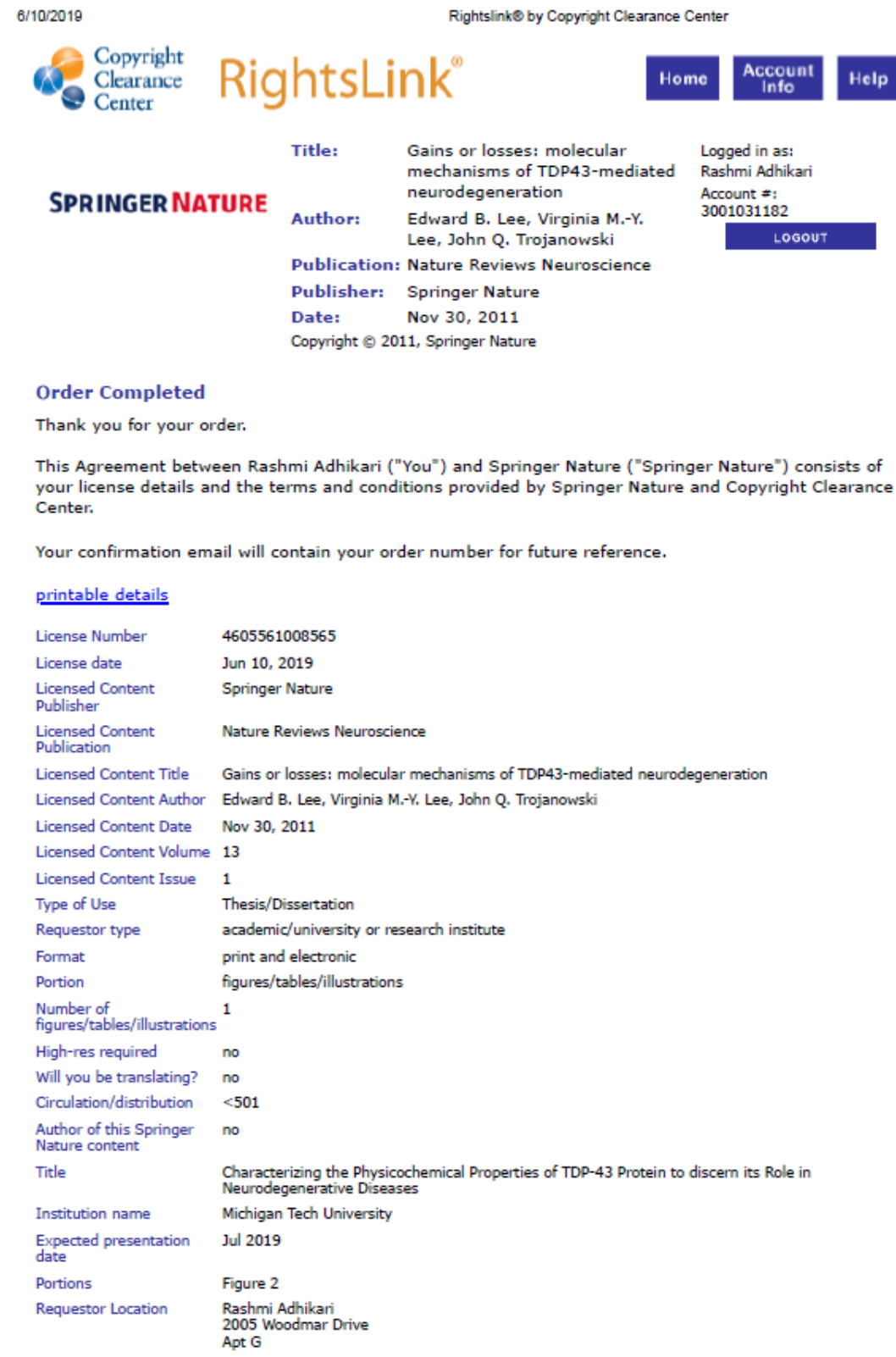

Title:

Gains or losses: molecular Rashmi Adhikari

SPRINGER NATURE

Author:

Edward B. Lee, Virginia M.-Y. Lee, John Q. Trojanowski

Publication: Nature Reviews Neuroscience

Publisher: Springer Nature

Date: Nov 30, 2011

Copyright @e 2011, Springer Nature

Order Completed

Thank you for your order.

This Agreement between Rashmi Adhikari ("You") and Springer Nature ("Springer Nature") consists of your license details and the terms and conditions provided by Springer Nature and Copyright Clearance Center.

Your confirmation email will contain your order number for future reference.

printable details 


\section{Permission for figure 4 in Chapter 3}

Permission to use figure for $\mathrm{PhD}$ Dissertation Inbox $\mathrm{x}$

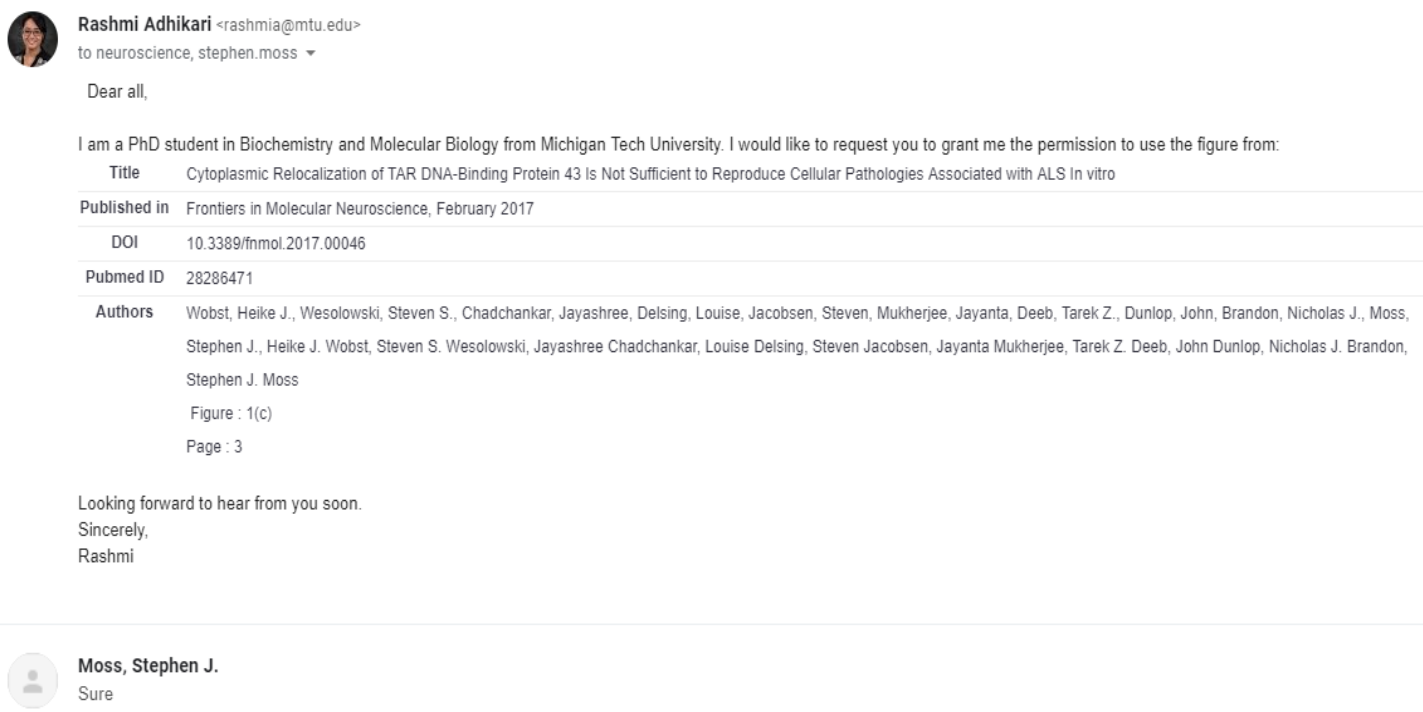




\section{Permission for figure 5 in Chapter 3}

\section{JOHN WILEY AND SONS LICENSE TERMS AND CONDITIONS}

Jun 02, 2019

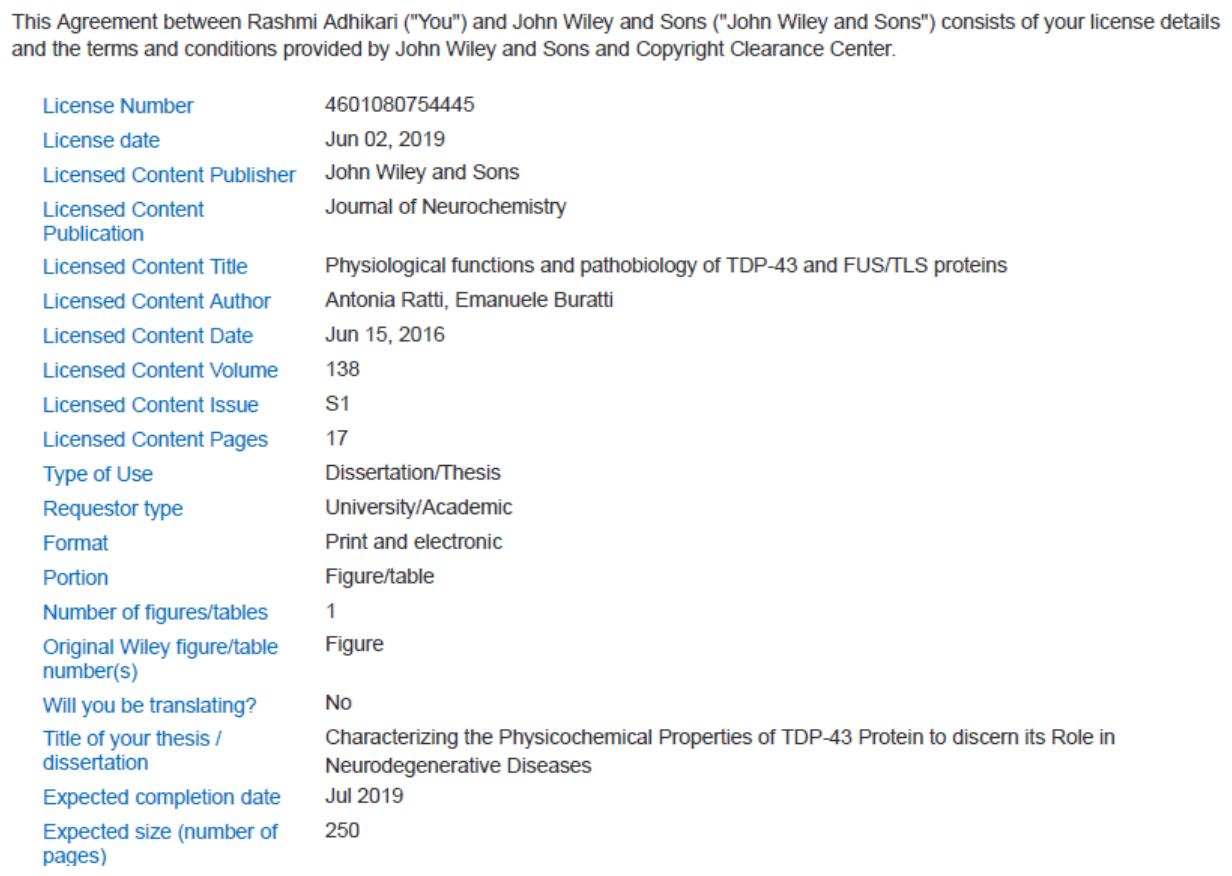


Permission for figure 4 and modeling figure in supporting information in chapter 4

Request to use modeling figure in Ph.D. dissertation Inbox $x$

Rashmi Adhikari <rashmia@mtu.edu>

to Nabanita, Ravindra, Ashutosh -

Dear Dr. Saikia and Professor Pandey,

\section{Ravindra Pandey <pandey@mtu.edu>}

to me, Nabanita, Ashutosh -

sure, rashmi.

ravi

Nabanita Saikia <nsaikia@mtu.edu>

to me, Ravindra, Ashutosh -

Dear Rashmi,

Okay with me as well.

Thanking you, 